\title{
Functional Nanocomposite Hydrogels Based on Cellulose Nanocrystals
}

\author{
Dissertation \\ for the award of the degree \\ "Doctor rerum naturalium" (Dr.rer.nat.) \\ of the Georg-August-Universität Göttingen
}

within the doctoral program "Wood Biology and Wood Technology" of the Graduiertenschule Forst- und Agrarwissenschaften (GFA)

\author{
submitted by \\ Xiaojie Wang \\ from P.R. China
}

Göttingen, 2020 


\section{Thesis Committee}

Prof. Dr. Kai Zhang, Abteilung Holztechnologie und Holzwerkstoffe, Georg-AugustUniversität Göttingen

Dr. Florian Rehfeldt, Drittes Physikalisches Institut - Biophysik, Georg-August-Universität Göttingen

Prof. Dr. Carsten Mai, Abteilung Holzbiologie und Holzprodukte, Georg-August-Universität Göttingen

\section{Members of the Examination Board}

\section{Reviewer:}

Prof. Dr. Kai Zhang, Abteilung Holztechnologie und Holzwerkstoffe, Georg-AugustUniversität Göttingen

Second Reviewer:

Dr. Florian Rehfeldt, Drittes Physikalisches Institut - Biophysik, Georg-August-Universität Göttingen

\section{Further members of the Examination Board:}

Prof. Dr. Carsten Mai, Abteilung Holzbiologie und Holzprodukte, Georg-August-Universität Göttingen

Date of the oral examination: July 31, 2020 


\section{Acknowledgement}

First and foremost, I would like to express my special thanks to my supervisor Prof. Dr. Kai Zhang for his invaluable guidance and support in completing my Ph.D study and related research. He helped me a lot during the research and writing this thesis. I am very lucky to pursue my Ph.D degree under the guidance of such a good advisor with patience, motivation and immense knowledge. This three and half years are definitely to be a precious and memorable time in my life.

Besides, I would like to extend my gratitude to other two supervisors in my thesis committee: Dr. Florian Rehfeldt and Prof. Dr. Carsten Mai, for their scientific advice, knowledge and many insightful discussions and suggestions. I also deeply appreciate their generous help in research facilities supporting during my Ph.D study.

Moreover, my sincere thanks also go to all my collaborators for their inspirational discussions, and generous research facilities providing. Special thanks to Dr. Gerhard Büttner for his help throughout my stay in Göttingen. I also greatly thank all my group fellows, for the stimulating discussions and for all the fun we had in the last three and half years. Here, I also thank China Scholarship Council (CSC) for the financial support for my Ph.D study in Germany.

Last but not the least, I would like to thank my parents, my brother, my friends, and my beloved husband - Heqin Huang for their unconditional love, spiritually support, and continuous encouragement. 


\begin{abstract}
Hydrogels are ubiquitous in nature, which are three-dimensionally (3D) crosslinked polymer networks with amounts of water inside. Naturally, they can be regarded as semi/solid showing intermediate properties of solid and liquid. Hydrogels have attracted growing interest in diverse applications, due to their excellent biocompatibility, permeability, and 3D network. With the development of polymer synthesis, great progress in tough hydrogels, and continuous emerging of advanced fabrication methods, hydrogels become promising functional materials. To further broaden their practical usages, great efforts have made to hydrogel functionalization, which mainly focused on both polymer network and architectures within hydrogels. Normally, functional hydrogels were fabricated from stimuli-responsive polymers, cleavable bonding, inhomogeneous or aligned microstructures, especially, functionalization by diverse nanoparticle composites.
\end{abstract}

In this study, cellulose nanocrystals (CNCs) as one of the bio-based natural nanoparticles were investigated in hydrogel functionalization owing to their excellent mechanical properties, facile surface modification and unique optical properties. CNCs and surface modified CNCs were introduced into synthetic hydrogels to ensure the implementation of specific functions of hydrogels.

CNCs and surface modified CNCs with methyl acrylamide groups (CNCs-MAm) were incorporated into thermal-responsive and solvent-driven bilayer hydrogel actuators (BHAs). The introduction of CNCs and CNCs-MAm significantly improved the mechanical properties of BHAs and ensured largely promoted lifting capability for them. The weight-lifting capability of BHAs was promoted from $\sim 800 \mathrm{wt} \%$ to $\sim 1800 \mathrm{wt} \%$ of their own polymer weight.

Furthermore, the CNCs with numerous carboxyl groups on the surface can apparently increase the spatial distinction of dynamic hydrogels. When ferric ions were introduced along with diffusion from outside to inside of hydrogels, the carboxyl groups would complex with ferric ions. This additional crosslinking retarded the penetration of ferric ions, increasing the spatial crosslinking difference. This promoted spatial distinction assisted in fabricating closed hollow 
hydrogels with tunable microstructures of the inner and outer walls. CNCs can accelerate the process of spatial separation to form the hollow interior in around 9 days of dialysis, whereas, about 20 days of dialysis for non-composite hydrogels. Distinguished from typical thermal controlled release system, this hollow hydrogel showed unique sustained release of hydrophilic small molecules at higher temperature. It would reach release equilibrium after only $\sim 24 \mathrm{~h}$ at $25^{\circ} \mathrm{C}$, while the release equilibrium was largely retarded to $\sim 200 \mathrm{~h}$ at $37^{\circ} \mathrm{C}$ as comparison.

In addition, as one-dimensional nanomaterials, CNCs and gold nanorods (GNRs) are widely used in optical materials due to their respective inherent natures: birefringence with accompanying light retardation in aligned $\mathrm{CNCs}$ and surface plasmon resonance (SPR) of GNRs. These properties of both nanorods were combined to generate synergistic and readily tunable structural colors of polymer films. The CNCs and GNRs are embedded either in the same hybrid composite films or in separated films after their unidirectional alignment from dynamic precursor hydrogels. By synergistically leveraging the optical features of CNCs and GNRs with diverse amounts in hybrid films or in stacked individual films, wide-ranging structural colors were realized, which is far beyond the limitation of the same films solely with aligned CNCs or GNRs. Increasing GNRs contents leads to promoted color red with enhanced light absorption at $520 \mathrm{~nm}$ and CNCs influence the overall phase retardation, giving distinctively structural colors. Furthermore, with angle adjustment between CNCs films and GNRs films using stacking/rotating technique, we further achieve facile and continuous color manipulation easily for color combinations. In one set of stacked films, light absorption wavelengths can traverse from roughly $500 \mathrm{~nm}$ to $650 \mathrm{~nm}$ solely by rotating GNRs film (0$180^{\circ}$ ). Tuning the adjustable synergism of the birefringence of CNCs and SPR of GNRs in one film or separate films provides great potential for structural colors, which enlightens new avenue for optical applications.

This thesis is a cumulative work including 3 publications. One of them was already published and two are under submission. The background, the objective of the study, results and discussion of these three publications and the conclusion are presented in Sections 1-4. 


\section{Zusammenfassung}

Hydrogele, die aus dreidimensionalen verzweigten Polymer-Netzwerken mit hohem Wasseranteil bestehen, sind in der Natur allgegenwärtig. Im Allgemeinen, können sie als halbfest angesehen werden, da sie sowohl feste als auch flüssige Eigenschaften aufweisen. Hydrogele erfreuen sich wachsenden Interesses bei verschiedensten Anwendungen, aufgrund ihrer hervorragenden Biokompartibilität, Permeabilität und ihres 3D-Netzwerkes. Mit der Weiterentwicklung der Polymer-Synthese, den großen Fortschritten bei zähen Hydrogelen und der kontinuierlichen Entwicklung fortschrittlicher Herstellungsverfahren, entwickelten sich Hydrogele $\mathrm{zu}$ vielversprechenden funktionellen Materialien. Um ihre praktischen Anwendungen auszuweiten, wurden große Anstrengungen bei der weiteren Funktionalisierung unternommen, die sich zu einem großen Teil auf Polymer-Netzwerke und zum anderen Teil auf die makroskopischen sowie mikroskopischen Architekturen der Hydrogelen fokussieren. Normalerweise werden funktionelle Hydrogele aus auf Reize ansprechenden Polymeren, spaltbaren Bindungen, inhomogene oder ausgerichteten Micro-Strukturen, insbesondere durch die Funktionalisierung diverser Nanopartikel-Kompositionen, hergestellt.

In der hier vorliegenden Arbeit wurden Cellulose Nanopartikel (engl. Cellulose nano crystals, kurz: CNCs), als Vertreter der biobasierenden natürlichen Nanopartikeln, in Hinblick auf ihre hervorragenden mechanischen Eigenschaften, ihrer leichten Oberflächenmodifikationen und ihrer einzigartigen optischen Eigenschaften, zur Darstellung funktionalisierter Hydrogele untersucht. Dafür wurden CNCs und oberflächenmodifizierte CNCs in synthetische Hydrogele implementiert, um so eine spezifische Funktionalisierung der Hydrogele zu erreichen.

CNCs und oberflächenmodifizierte CNCs mit Methyl-Accrylamid-Funktionalitäten (kurz CNCs-MAm) wurden in thermisch und lösungsmittelbetriebene Doppelschicht-Hydrogele (engl. Bilayer hydrogel actuators, kurz: BHAs) eingebaut. Dies führte zu einer drastischen Verbesserung der mechanischen Eigenschaften der BHAs und sorgte für eine erhöhten Hebefähigkeit. Die Hebefähigkeit der BHAs konnte von $800 \mathrm{wt} \%$ auf $1800 \mathrm{wt} \%$ ihres Polymergewichtes erhöht werden. 
Des Weiteren können CNCs mit zahlreichen oberflächlichen Carboxyl-Gruppen die räumliche Abtrennung von dynamischen Hydrogelen sichtlich erhöhen. Sobald Eisen-Ionen durch Diffusion von außen in innere der Hydrogele gelangen, werden diese durch Carboxyl-Gruppen komplexiert. Diese zusätzliche Vernetzung verzögert das Eindringen der Eisen-Ionen, indem es die räumliche Vernetzungsdifferenz erhöht. Diese geförderte räumliche Unterscheidung trug entscheidend zur Herstellung von geschlossenen hohlen Hydrogelen, mit veränderlichen mikro-Strukturen der inneren und äußeren Wände, bei. Damit können CNCs den Prozess der räumlichen Trennung bei der Bildung eines hohlen Innenraumes auf etwa neun Tage Dialyse beschleunigen, wohingegen es bei nicht modifizierten Hydrogelen einer Dialyse-Zeit von 20 Tagen bedarf. Abhebend von typischen Systemen mit thermisch kontrollierbarer Freisetzung zeigte dieses hohle Hydrogel eine einzigartige nachhaltige Freisetzung kleiner hydrophiler Moleküle bei höheren Temperaturen. Während das Freisetzungs-Gleichgewicht normalerweise nach $\sim 24 \mathrm{~h}$ bei $25^{\circ} \mathrm{C}$ erreicht wird, konnte es in diesem Falle auf $\sim 200 \mathrm{~h}$ bei $37^{\circ} \mathrm{C}$ verzögert werden.

Neben den bisher genannten, werden CNCs und Gold Nano-Stäbchen (engl. gold nanorods, kurz: GNRs), als eindimensionale Nano-Materialien in optischen Materialien, wegen ihrer jeweiligen inhärenten Natur, Doppelbrechungen mit begleitender Lichtverzögerung bei ausgerichteten CNCs und Oberflächenplasmonenresonanz (engl. surface plasmon resonance, kurz SPR) von GNRs genutzt. Die Eigenschaften der beiden Nano-Stäbchen wurden kombiniert, um synergetische und leicht veränderbare strukturelle Farben in Polymer-Filmen zu generieren. Dafür werden die CNCs and GNRs entweder in die gleichen hybriden Verbundsfilm eingebettet oder in zwei separaten Filmen nach der unidirektionalen Ausrichtung der dynamischen Vorläufer-Hydrogelen. Durch die synergetische Nutzung der optischen Eigenschaften der CNCs und der GNRs mit unterschiedlichen Mengen in Hybrid-Filmen bzw. gestapelten Einzelfilmen, konnten weitreichende strukturelle Farben realisiert werden, die weit über die Beschränkungen der gleichen Filme allein mit ausgerichteten CNCs oder GNRs hinausgeht. Das Erhöhen des GNRs-Gehaltes führt zu einer roten Farbe mit einer erhöhten Lichtabsorption bei $520 \mathrm{~nm}$, wohingegen CNCs die allgemeine Phasenverzögerung beeinflussen, was zu ausgeprägten Strukturfarben führt. Darüber hinaus lassen die durch eine 
Verstellung der Winkel zwischen CNC und GNR-Filmen mittels Stapel-/Drehtechnik eine einfache und kontinuierliche Farbmanipulation für weitere Farbkombinationen erreichen. In einem Aufbau gestapelter Filme konnten die Lichtabsorptionswellenlängen einen Bereich von $500 \mathrm{~nm}$ bis $650 \mathrm{~nm}$ durchlaufen durch die alleinige Rotation der GNR-Filme $\left(0^{\circ}-180^{\circ}\right)$. Die Abstimmung der schaltbaren Synergien der Doppelbrechungen der CNC und der SPR der GNR in einem Film eröffnet ein großes Potential an Strukturfarben, die wiederum ein großes Spektrum für optische Anwendungen generieren.

Bei der hier vorliegenden Arbeit handelt es sich um eine kumulative Arbeit, die drei Publikationen umfasst. Eine davon ist bereits veröffentlicht, die anderen beiden wurden eingereicht. Der Hintergrund, die Ziele dieser Studie, die Resultate und die Diskussion dieser drei Veröffentlichungen und die Zusammenfassung werden nachfolgen in den Abschnitten 1 bis 4 dargestellt. 


\section{Table of Contents}

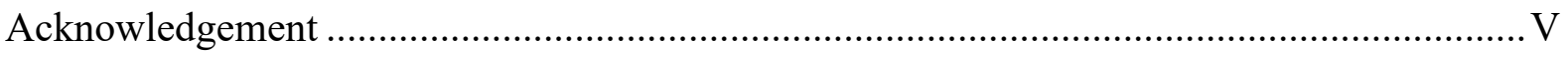

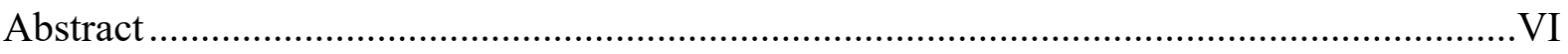

Zusammenfassung ................................................................................................ VIII

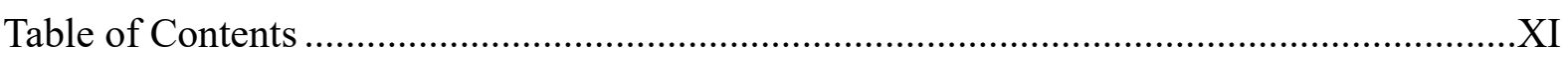

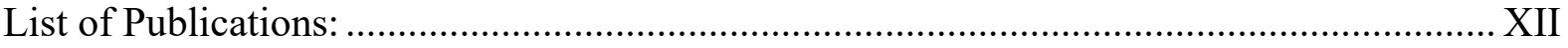

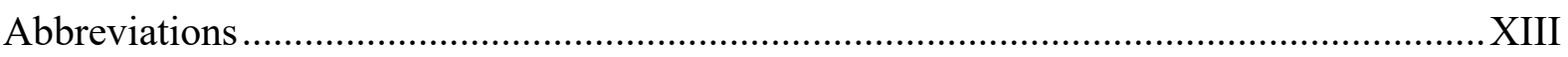

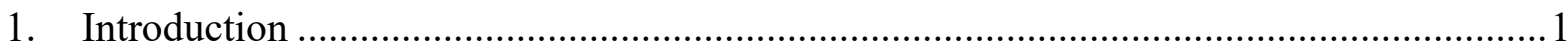

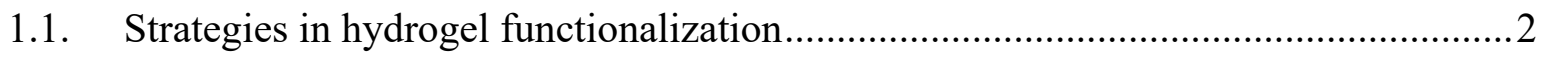

1.1.1 Hydrogel functionalization with rational design in polymer network .....................2

1.1.2 Hydrogel functionalization with specific architectures ...........................................5

1.2. Hydrogel functionalization with polysaccharide nanocrystals.................................... 11

1.2.1 Cellulose nanocrystals (CNCs) and their applications in hydrogels .....................12

1.2.2 Chitin nanocrystals (ChNCs) and their applications in hydrogels ........................17

1.2.3 Starch nanocrystals (SNCs) and their applications in hydrogels...........................20

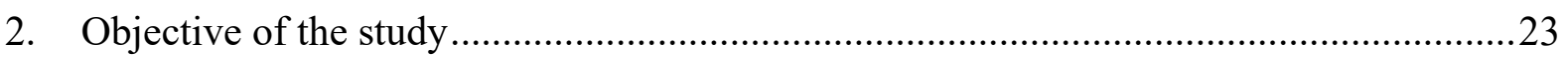

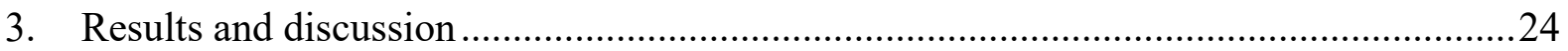

3.1. Bilayer hydrogel actuators with programmable and precisely tunable motions .......24

3.2. Temperature-Responsive, Manipulable Cavitary Hydrogel Containers by

Macroscopic Spatial Surface-Interior Separation.............................................................30

3.3. Structural Colors by Synergistic Birefringence and Surface Plasmon Resonance ...36

4. General conclusion and perspectives.........................................................................4

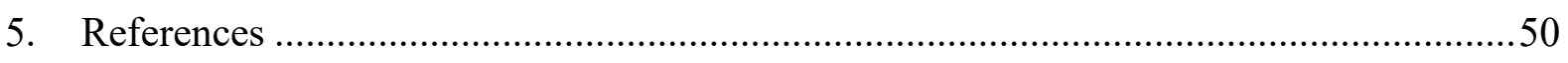

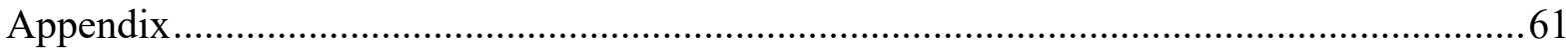

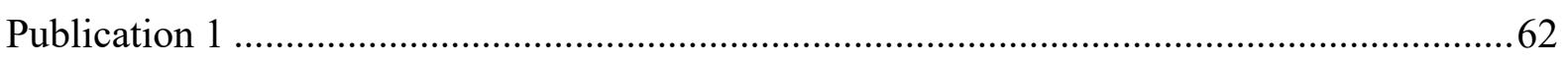

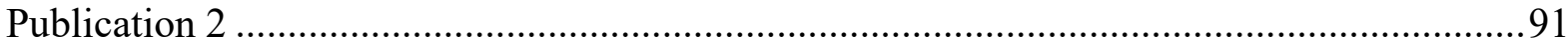

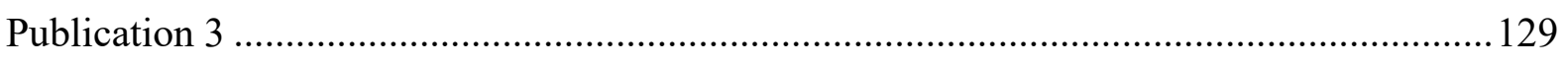

Curriculum vitae 


\section{List of Publications:}

Publication 1

Multi-responsive bilayer hydrogel actuators with programmable and precisely tunable motions.

Xiaojie Wang, Heqin Huang, Huan Liu, Florian Rehfeldt, Xiaohui Wang and Kai Zhang* Macromolecular Chemistry and Physics 2019, 220 (6), 1800562. (Front cover)

Publication 2

Temperature-Responsive, Manipulable Cavitary Hydrogel Containers by Macroscopic Spatial Surface-Interior Separation.

Xiaojie Wang, Yang Yang, Heqin Huang, Kai Zhang*

submitted

Publication 3

Structural Colors by Synergistic Birefringence and Surface Plasmon Resonance.

Xiaojie Wang, Dan $\mathrm{Xu}$, Bea Jaquet, Yang Yang, Jiaxiu Wang, Heqin Huang, Ye Chen, Christoph Gerhard, Kai Zhang*

submitted 


\section{Abbreviations}

2D

$3 \mathrm{D}$

BHAs

CDI

ChNCs

CIE

CNCs

CNTs

DMA

GNRs

HHs

LAP

LCST

L-SPR

PAAm

PBA

PEG

PHEAm

PNIPAm

SEM

SNCs

SPR

TEM

TEMPO

T-SPR

UV-Vis-NIR
Two dimensional

Three dimensional

Bilayer hydrogel actuators

1,1'-carbonyldiimidazole

Chitin nanocrystals

Commission internationale de l'éclairag

Cellulose nanocrystals

Carbon nanotubes

Dopamine methyl acrylamide

Gold nanorods

Hollow hydrogels

Lithium phenyl-2,4,6-trimethylbenzoylphosphinate

Lower critical solution temperature

Longitudinal surface plasmon resonance

Polyacrylamide

Phenylboronic acid

Poly(ethylene glycol)

Poly(N-hydroxyethyl acrylamide)

Poly(N-isopropylacrylamide)

Scanning electron microscopy

Starch nanocrystals

Surface plasmon resonance

Transmission electron microscopy

2,2,6,6-tetramethylpiperidin-1-oxyl

Transverse surface plasmon resonance

Ultraviolet-Visible-Near Infrared 


\section{Introduction}

Hydrogels are three-dimensionally (3D) crosslinked polymer networks with amounts of water in the highly porous structure. ${ }^{1}$ The crosslinked polymer networks endow hydrogels with the properties of soft and elastic solid, while the high-water content in hydrogels give rise to liquidlike properties, such as good permeability to various chemicals, plastic behaviors and adjustable optical characteristics. ${ }^{2}$ In addition, hydrogels have unique properties, such as the responsiveness and swelling, brought by their tailorable polymer network and exchangeable aqueous system. ${ }^{3}$ All these features make hydrogels promising semi/solid materials with diverse practical applications including soft actuators, ${ }^{4}$ responsive release devices, ${ }^{5}$ supercapacitors, ${ }^{6}$ optical materials, ${ }^{7}$ and so on. ${ }^{8-16}$

With the development of polymer synthesis, great progress in tough hydrogels and continuous emerging of advanced fabrication methods, hydrogels become promising functional materials for practical usages. Considering the presence of both architectures (macro- and microstructure) and main components in hydrogels (polymers and crosslinking sites), various approaches were created to introduce diverse functions to hydrogels. Normally, functional hydrogels were fabricated from stimuli responsive polymers, cleavable bonding, directional aligned microstructures and variety of inorganic, organic as well as metal nanocomposites.

Therefore, the functionalization strategies for hydrogels are mainly focused on two categories, hydrogel functionalization by polymer network design and hydrogel functionalization by architecture design (Figure 1). 


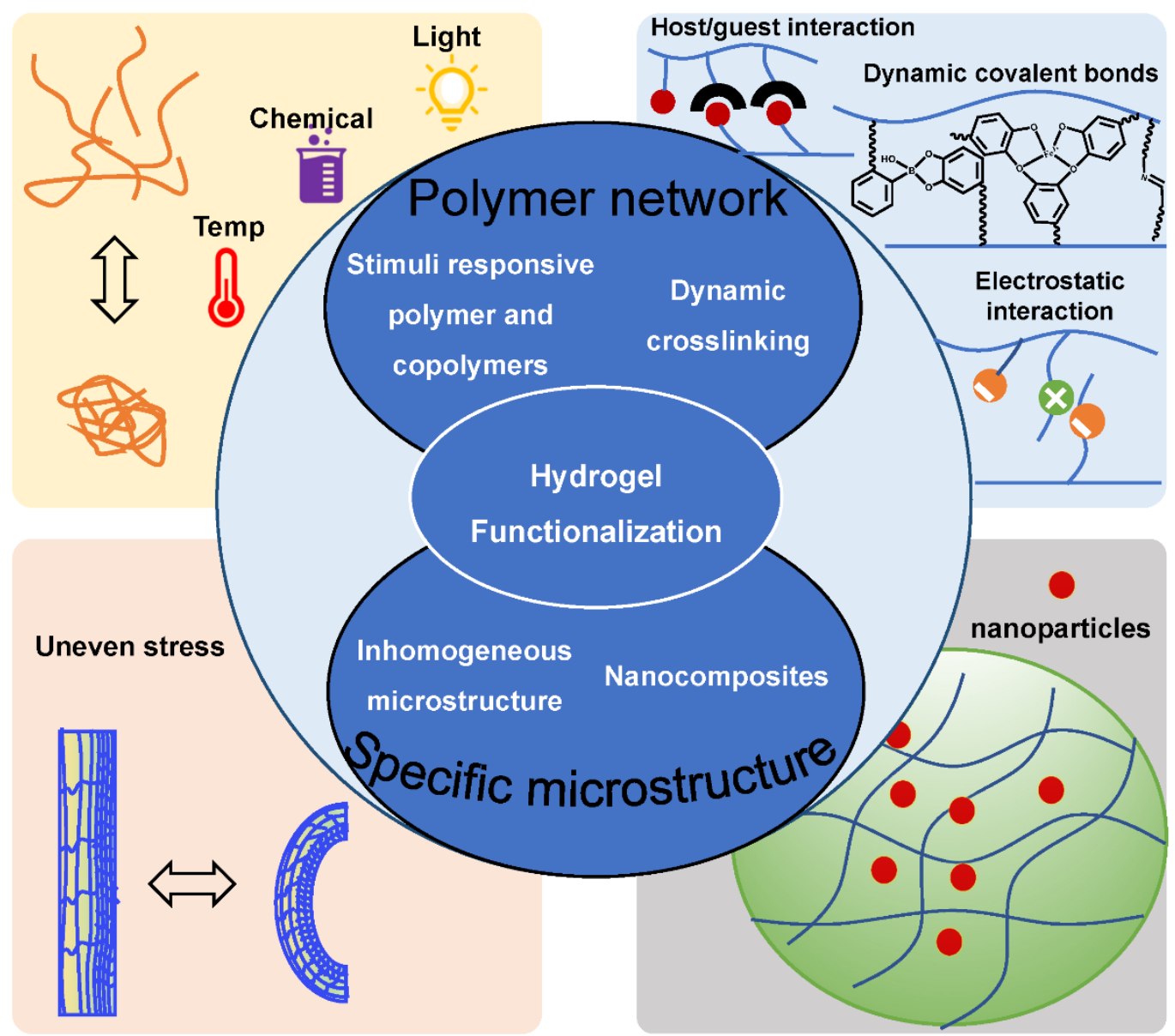

Figure 1. Diverse approaches for hydrogel functionalization.

\subsection{Strategies in hydrogel functionalization}

\subsubsection{Hydrogel functionalization with rational design in polymer network}

Hydrogels are crosslinked polymer chains swollen in water. Indeed, the properties of hydrogels highly depend on the polymer backbone and their crosslinking kinetics. By now, various polymers with diverse functions have been designed in functional hydrogels with all kinds of crosslinking kinetics, including static bonding and dynamic bonding. Great efforts were continuously targeted on the polymer network design to achieve hydrogel functionalization.

\subsubsection{Stimuli-responsive polymers in hydrogels}

Hydrogels are able to swell and shrink in aqueous environment. The changes in the affinity of polymer chains to water would have significant influence on the water content of hydrogels, leading to remarkable volume change. Diverse functional hydrogels were constructed basing 
on this volume change. Poly(N-isopropylacrylamide) (PNIPAm) hydrogels were prepared with the transformation of hydrophilic to hydrophobic in response to temperature rise, which could serve as temperature sensors. To overcome the inevitable swelling for injectable hydrogels, Takamasa Sakai's group created a series of non-swellable hydrogels comprising of hydrophilic four-arm poly(ethylene glycol) (PEG) and thermoresponsive units. By regulating the proportion of their content, the swelling of the hydrophilic polymer and thermal-induced shrinking can achieve a balance, resulting in non-swellable hydrogels (Figure 2a). ${ }^{17,18}$ Besides, polyelectrolyte hydrogels can be adopted to detect changes in $\mathrm{pH},{ }^{19}$ ion concentration ${ }^{20}$ and electrical potential. ${ }^{21}$ With the development of polymer synthesis, many copolymers were prepared which fulfilled the multi responsiveness. For example, in situ copolymerization of PNIPAm and polyacrylic acid has been used to fabricate temperature, $\mathrm{pH}$, ionic responsive hydrogels. ${ }^{22}$ Due to the relatively static network with large volume, Zhao et al. designed a super moisture absorbent gels composing of hygroscopic polypyrrole chloride penetrating in temperature-responsive polymeric network of PNIPAm. ${ }^{23}$ This system enabled the successive processes of moisture capture, liquefication by hygroscopic polypyrrole chloride, water storage in PNIPAm hydrogel network, then water release upon raising temperature (Figure 2b).

a

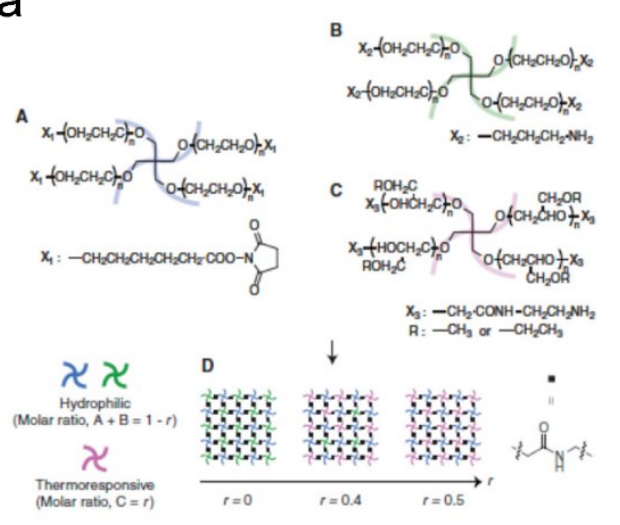

C

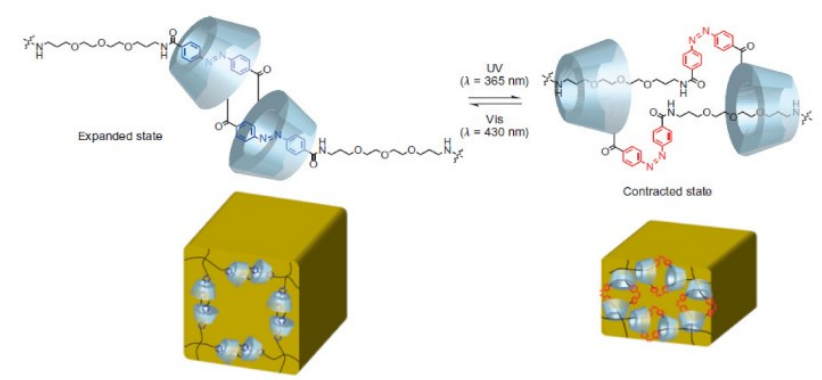

b

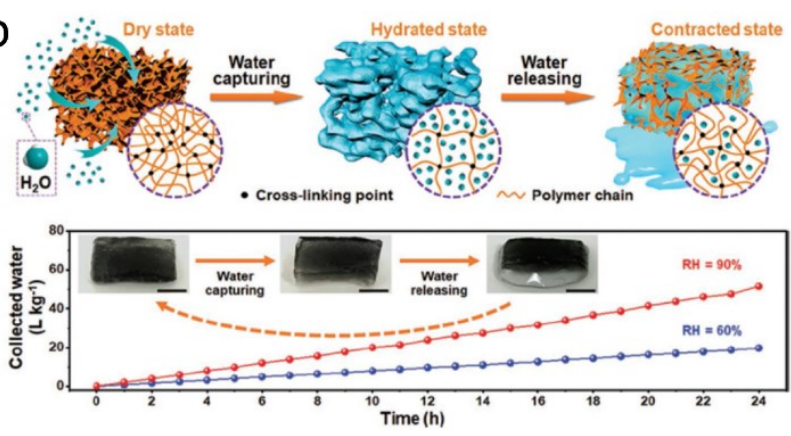

d

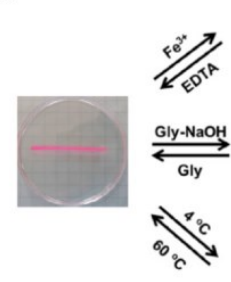

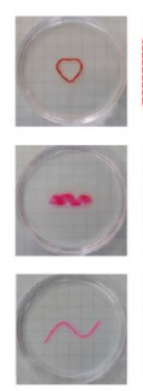

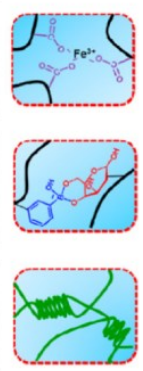


Figure 2. Hydrogel functionalization with rational design in polymer network. (a) Nonswellable hydrogel. (Reproduced from KAMATA et al. ${ }^{17}$ Copyright from Science Publishing Group 2014) (b) Super moisture absorbent gels. (Reproduced from ZHAO et al. ${ }^{23}$ Copyright from WILEY-VCH 2019) (c) Light responsive artificial molecular muscles with isomerization induced expansion/contraction. (Reproduced from IWASO et al. ${ }^{32}$ Copyright from Nature Publishing Group 2016) (d) Supramolecular hydrogel with multi-stimuli and multi-shape memory effect. Reproduced from LE et al. ${ }^{34}$ Copyright from American Chemical Society 2017)

\subsubsection{Dynamic crosslinking in hydrogels}

In addition, many reversible chemical bonds, physical interactions also contributed to functional hydrogels, such as redox responsive disulfide bonds, ${ }^{24}$ host-guest interactions between beta-cyclodextrin and ferrocene, ${ }^{25,26}$ light-responsive azobenzene and spiropyran, ${ }^{27-}$ ${ }^{29} \mathrm{pH}$-sensitive boronic ester bonds ${ }^{30}$ and ionic bonds. ${ }^{31}$ Based on those responsive compounds, Iwaso et al. reported fast light-responsive artificial molecular muscles in both wet and dry state, which was built from rotaxane-based [c2] daisy chain unit crosslinked four-arm PEG (Figure 2c). ${ }^{32}$ Hydrogel, as well as its xerogel, were contracted with ultraviolet light irradiation due to the trans to cis isomerization of azobenzene which stretched the polymer chains to shrink. More importantly, the response speed of the [c2] daisy chain xerogel was very fast with speed more than 10,800 times faster than that of the hydrogel. Peter X. Ma reported stimuli-responsive supramolecular hydrogels with high extensibility and fast self-healing achieved by the dynamic catechol-Fe ${ }^{3+}$ bond. ${ }^{33}$ Moreover, multi-crosslinking kinetics can cooperate within hydrogels which ensures other fascinating applications. Hydrogels with multi-shape memory functionalities were created by using three programmable reversible associations comprising of phenyl boronic ester bonds, acrylic acid/ferric ions complexes and coil-helix transition of agar (Figure 2d). ${ }^{34}$

In addition to the polymers and crosslinking kinetics, the 3D network can hold various watersoluble compounds, such as ionic salts, nutrients and biomolecules. With the addition of ionic salts, ionically conductive hydrogels were synthesized. ${ }^{35}$ Based on this, a great quantity of electronic devices was developed, including diodes, ${ }^{36}$ signal transmitters ${ }^{13}$ and power 
sources. ${ }^{37}$ Moreover, the addition of ionic salts would give rise to the anti-freezing property to electronic devices, which would benefit to practical usage at low temperature conditions. ${ }^{38}$ In addition, the freely transportation of nutrients makes hydrogels ideal materials for cell culture. $^{39}$

\subsubsection{Hydrogel functionalization with specific architectures}

With the rapid development of advanced fabricating methods for hydrogels, hydrogels functionalization got large progress within both mechanical strength and diverse functions satisfying more complicated applications. Through the freeze-thaw method, ${ }^{40}$ uniaxial mechanical stretching, ${ }^{41}$ photolithography, ${ }^{42} 3 \mathrm{D}$ printing, and electrospinning, ${ }^{43}$ hydrogels succeeded to have programmed macro- or microstructures and diverse shapes, which greatly expanded their applications.

\subsubsection{Inhomogeneous architecture design of hydrogels}

Constructing inhomogeneous microstructure within hydrogels is significant in various applications, such as self-patterning hydrogels, morphing hydrogels and actuators with diverse inhomogeneous deformations. ${ }^{44}$ The straightforward way to get inhomogeneous hydrogel is to combine hydrogels with differed swelling behaviors, for example, the bilayer hydrogels. Duan and coworkers reported a bilayer hydrogel constructed by tight adhered positively charged chitosan with negatively charged cellulose/carboxymethylcellulose layers. The swelling ratio of each layer was different, as a result, the bilayer hydrogel gained bidirectional rolling with pH change (Figure 3a). ${ }^{45}$

Furthermore, the inhomogeneity within hydrogels can also be fabricated via setting gradient polymerization degree or crosslinking density. By using photomask, stress inhomogeneity and its distribution could be programmed in hydrogels on demand, offering the possibility to regulate hydrogel behaviors from microscopic to macroscopic scale. Wang and coworkers synthesized a series of programmable deformations of patterned hydrogel sheets with various dome-like structure as the building block, which can buckle into desired directions with the help of a pre-swelling process (Figure 3b). ${ }^{46}$ Palleau et al. designed hydrogel with reversible 
patterning and actuation behavior by the assistance of electrical ion printing. Through this method, the $\mathrm{Cu}^{2+}$ can be programmed to print to the polyelectrolyte hydrogels at certain regions. The ions printed parts in hydrogels had larger modulus than the original ones, leading to anisotropic actuation when transferred to organic solvents (Figure 3c) ${ }^{47}$ In particular, due to the good permeability of hydrogels, ions, such as $\mathrm{Cu}^{2+}, \mathrm{Ca}^{2+}$ and $\mathrm{Fe}^{3+}$, can penetrate into hydrogels and chelate with various chemical groups. Their diffusion processes from outside to inside would generate gradient crosslinking density, which can give new avenue to hydrogel functionalization basing on this post-treatment. ${ }^{48,49}$

Natural biological soft tissues, such as muscles, can be regarded as living hydrogels, which possess ordered hierarchical structures that range in scale from the molecular to the macroscopic. Such unique hierarchical structures endow living organisms with anisotropic mechanical toughness and functionality, thereby adapting them to complicated usages in external environments. Inspired by this, Mredha and coworkers reported a method to prepare anisotropic hydrogels with perfectly aligned hierarchical fibrous structures. ${ }^{50}$ Air drying diluted physical hydrogel within confined length generated high tensile stress along the length direction, which can orient the polymer chains and form multiscale fibrous structures. This aligned hierarchical fibrous structures can well-retain in the re-swollen gel. The mechanical properties of hydrogel were comparable with those of natural ligaments (Figure 3d). Precisely control the stress alignment within stimuli-responsive hydrogels would guide their motions. Liu et al. reported an electrospinned fibrous bilayer system comprising thermoplastic polyurethane (TPU) and cross-linked PNIPAm fibers (Figure 3e). ${ }^{51}$ The TPU and PNIPAm fibers were aligned in various angles as passive and active layers, respectively. As a result, these fiber mattes displayed pre-programmed rolling motion with changing the surrounding temperature. It demonstrated reversible bending, coiling, rolling and twisting movements in certain directions for up to 50 cycles. Lei et al. reported a multifunctional skin-like sensor adopting the $3 \mathrm{D}$ printed thermal responsive hydrogel of poly( $\mathrm{N}, \mathrm{N}$-dimethylacrylamide), which realized the sensing of skin temperature and human motion. ${ }^{12}$ In particular, this hydrogel was printed with grid microstructures into the capacitor circuit, which can magnify capacitive area variations on external stimuli. 
a

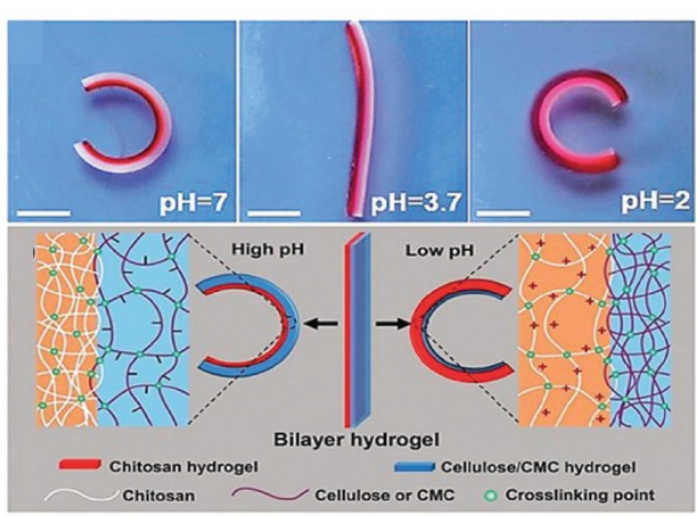

b

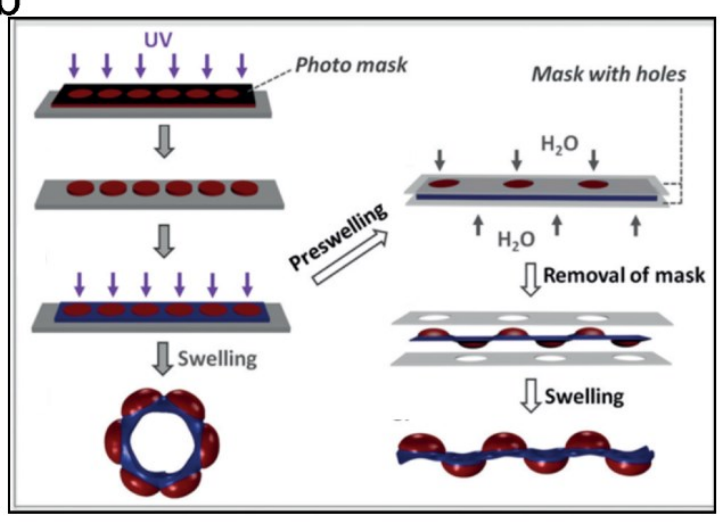

C

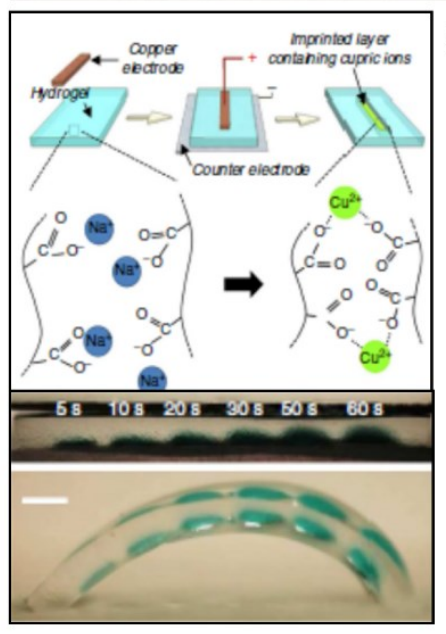

e

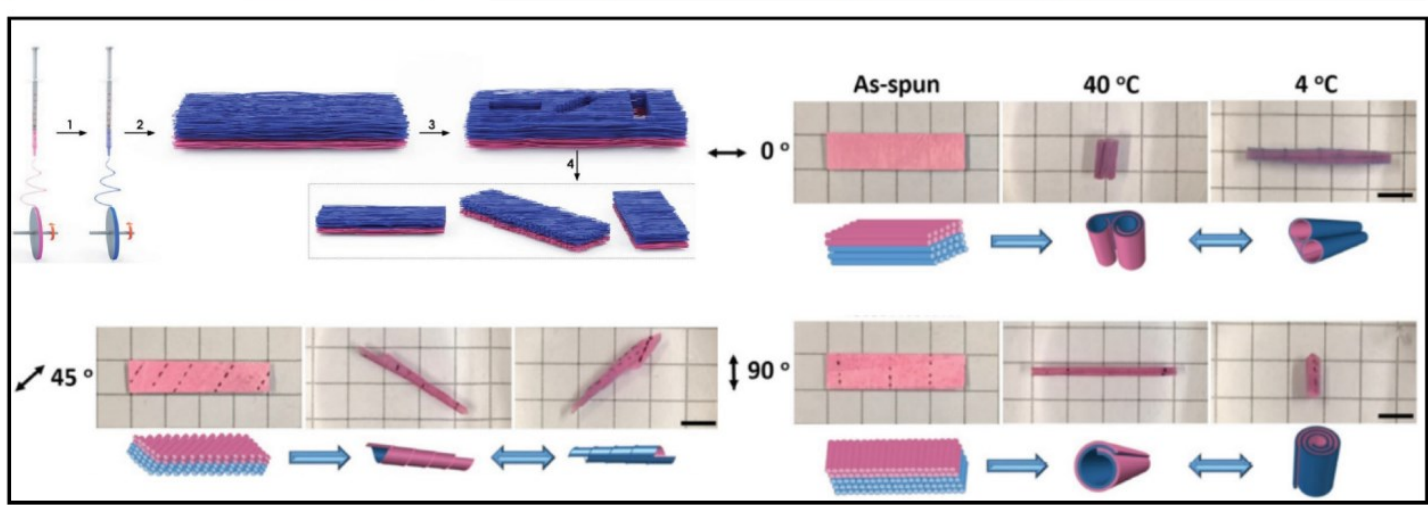

Figure 3. Hydrogel functionalization by programming inhomogeneous microstructure. (a) Bilayer hydrogel design. (Reproduced from DUAN et al. ${ }^{45}$ Copyright from Royal Society of Chemistry 2017) (b) Photomask induced patterned hydrogel sheets with programmable deformations. (Reproduced from WANG et al. ${ }^{46}$ Copyright from WILEY-VCH 2017) (c) Polyelectrolyte hydrogels with ion printed sites. (Reproduced from PALLEAU et al. ${ }^{47}$ Copyright from Nature Publishing Group 2013)(d) Anisotropic hydrogels with perfectly aligned hierarchical fibrous structures. (Reproduced from MREDHA et al. ${ }^{50}$ Copyright from WILEY-VCH 2018) (e) Electrospun fibrous bilayer system ensured programmable motion 
direction. (Reproduced from LIU et al. ${ }^{51}$ Copyright from WILEY-VCH 2015)

\subsubsection{Incorporation of nanoparticles in hydrogels}

Benefiting from the fast development of nanotechnology, many polymeric organic, inorganic, and metal-based nanomaterials, such as micelles, nanogels, nanoclays, carbon nanomaterials, and diverse nanometals, have been extensively applied into functional hydrogels. ${ }^{52,53}$ Indeed, it is well known that nanocomposites with well-dispersed nanoparticles get improved mechanical properties because of the load transfer from the polymer matrix to reinforcement fillers. ${ }^{54} \mathrm{~A}$ variety of nanocomposite hydrogels emerged with strong mechanical strength and other fascinating properties originating from the growing nanotechnology. Nanocomposite hydrogels were prepared with diverse approaches. In general, those nanomaterials would serve as blending nanofillers and multifunctional crosslinking points (Figure 4).

Mixing nanofillers. Firstly, the nanomaterials can be incorporated as normal nanofillers. Indeed, the nanoscale dimensions allow high surface-to-volume and aspect ratios, which increase the polymer network entanglement acting as bridges, leading to strengthened mechanical properties. In the microscale and macroscale, the fracture and pullout of nanofillers can work as the energy dissipation system to toughen hydrogels. ${ }^{55}$

Multifunctional crosslinking nanomaterials. More importantly, the nanomaterials can work as nanocrosslinkers for hydrogels through physical or chemical bonds. Those nanomaterials naturally have specific functional groups or surface charges on their surfaces, which can interact with polymers and allow further chemical modifications to introduce diverse chemical groups as well as polymer chains. In hydrogels, nanocrosslinkers could build multiple connections between two nanocrosslinkers, therefore their stepwise breaking or detachment could dissipate the elastic potential energy stored in the deformed polymer networks. ${ }^{56}$ Hence, the nanocrosslinkers can effectively retard the propagation of local defects in hydrogels, leading to toughened hydrogels. However, those sequential breaking polymer chains are irreversible for covalent bonds incorporated nanoparticles, which seriously weaken the longlasting usage for hydrogels. Therefore, many reversible interactions, such as ionic bonds, ${ }^{57}$ 
host-guest interactions, ${ }^{58}$ metal-ligand complexations, ${ }^{59}$ were introduced to nanoparticles and applied as the recoverable energy dissipation system.

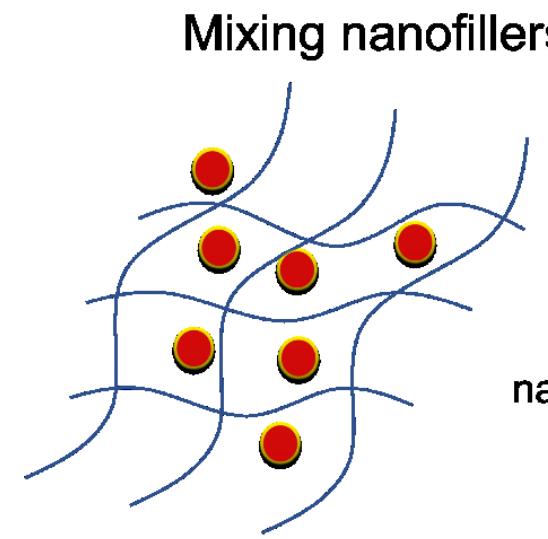

\section{Crosslinking nanofillers}

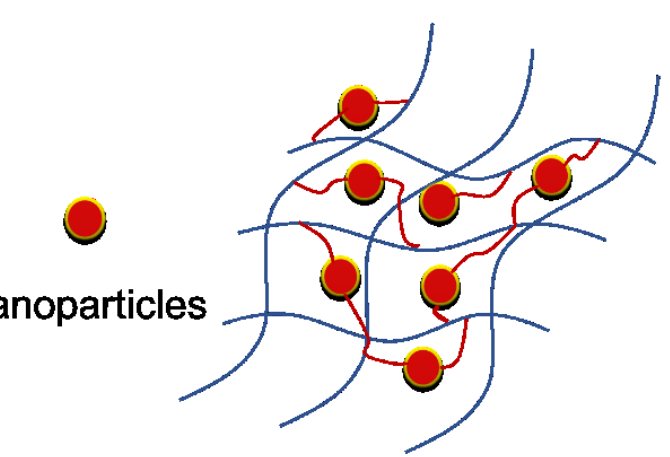

Figure 4. Incorporation forms of nanoparticles in the hydrogel network.

\subsubsection{Various types of nanocomposite hydrogels and their applications}

The ascendant nanotechnology continuously provides various nanoparticles to functional hydrogels. Those nanoparticles arise from abundant sources with unique properties.

Polymeric organic nanoparticles composite hydrogels. A large number of polymeric nanoparticles, including micelles, nanogels, dendrimers and liposomes, were investigated in hydrogel functionalization. ${ }^{60}$ In particular, those nanocomposite hydrogels got wide applications in drug delivery field, due to their ability to entrap hydrophobic or hydrophilic drugs. For example, Lampe and coworkers entrapped poly(lactic-co-glycolic acid) microparticles to PEG hydrogel, which can release two different neurotropic factors in different regions of the brain. ${ }^{61}$

Inorganic nanoparticles composite hydrogels. There are many kinds of inorganic nanoparticles, including carbon-based nanoparticles (carbon nanotubes (CNTs), graphene, fullerene and nanodiamonds), ceramic nanoparticles (silica nanoparticles, titania nanoparticles, nanoclays, layered double hydroxides, etc.) and quantum dots. Numerous functional nanocomposite hydrogels were derived from these nanoparticles. 
CNTs and graphene got wide applications owing to their high mechanical strength, outstanding electrical conductivity and special optical performance. ${ }^{62}$ They were surface modified with carboxyl groups, amine groups, hydroxyl groups or other hydrophilic polymer chains to improve their hydrophilicity before incorporated into hydrogel matrix. ${ }^{63-65} \mathrm{CNTs}$ can reinforce hydrogels and gave them the ability of responsiveness to external electrical or temperature. Sun and coworkers developed a conductive polyacrylamide (PAAm) hydrogel with high strain sensitivity, self-recovery, good stretchability and mechanical strength via incorporating oxidized multi-walled CNTs. The oxidized multi-walled CNTs were functionalized with gelatin via hydrogen bonding between carboxyl groups on CNTs and hydroxyl, carboxyl groups in gelatin chains, leading to good dispersion in hydrogel network. All of those physical interactions between the CNTs, gelatin and PAAm chains contributed to the high mechanical performance. Besides, the introduction of CNTs could construct stable conductive pathways within the hydrogel, which endowed hydrogel with good strain sensitivity displaying stable and repeatable electrical signals. ${ }^{66}$

Intensive investigation on silicates-based nanoparticles was given to the well-known silica nanoparticle and the layered silicate nanoparticles, such as nanoclays, layered sodium silicates and layered double hydroxides. The nanoclays are anisotropic and plate-like with high aspect ratio, which are widely used in tissue adhesive nanocomposite hydrogels with enhanced mechanical strength. ${ }^{67} \mathrm{Li}$ and coworkers synthesized an ultra-stiff and thermo-responsive nanocomposite hydrogel with nanoclays crosslinked PNIPAm. The synthesized hydrogel demonstrated high tensile modulus (approximately $3500 \mathrm{kPa}$ ) and strength (approximately $1700 \mathrm{kPa}$ ). The nanocomposite hydrogel possessed well-defined, thermo-responsive swelling and deswelling, and was promising used as scaffold materials. ${ }^{68}$

Metal-based nanoparticle composite hydrogels. Numerous metallic nanoparticles were synthesized including gold nanoparticles, silver nanoparticles and metal-oxide nanoparticles, such as iron oxide $\left(\mathrm{Fe}_{3} \mathrm{O}_{4}, \mathrm{Fe}_{2} \mathrm{O}_{3}\right)$, which can be used to construct nanocomposite hydrogels for diverse applications. These metallic nanoparticles have extraordinary physical properties, including electrical and thermal conductivity (gold nanoparticles), magnetic properties (iron- 
oxide nanoparticles) as well as antimicrobial properties (silver nanoparticles). Therefore, nanocomposite hydrogels with these metallic nanoparticles attracted wide attention on photo imaging, drug-delivery systems, soft electronics, actuators, and sensors. ${ }^{69}$ For example, Sun et al. reported an anisotropic hydrogel actuator achieving earthworm-like directed peristaltic crawling. ${ }^{70}$ The cylindrical hydrogel comprised of gold nanoparticles for photothermal conversion, thermo-responsive polymer network for changing the electrical permittivity in the interior, and cofacially oriented 2D electrolytes (titanate nanosheets) to synchronously switch the anisotropic electrostatic repulsion. When the hydrogel was irradiated with visible light, it spatiotemporally expands immediately with a large degree in a constant volume. When the irradiation point moving along the cylindrical gel axis, the hydrogel experiences peristaltic crawling because of the fast, sequential expansion and contraction.

However, there are still many limitations for practical applications of the current nanomaterials in hydrogels, such as the weak mechanical properties for polymeric nanoparticles, difficult surface modification for carbon-based nanoparticles, dispersion stability for silicates nanoparticles, and the concerns for heavy metal safety. Due to rising concerns over sustainability and environmental safety, many efforts have been devoted to developing biobased and eco-friendly nanoparticles with excellent physical and chemical properties.

\subsection{Hydrogel functionalization with polysaccharide nanocrystals}

There has been growing interest in developing natural polysaccharide nanocrystals for functional hydrogels, due to their unexhausted sources, excellent biocompatibility, unique physical and chemical properties. ${ }^{71}$ Polysaccharide nanocrystals have many virtues including high modulus (greater than $100 \mathrm{GPa}$ ), high specific surface area (greater than hundreds of $\mathrm{m}^{2}$ $\left.\mathrm{g}^{-1}\right)$, high aspect ratio, plentiful surface functional groups, and selectable morphologies. ${ }^{72}$ Polysaccharide nanocrystals are mainly comprised of rod-like cellulose nanocrystals, chitin nanocrystals and platelet-like starch nanocrystals. They are rigid, biodegradable, biocompatible and renewable nanoparticles with high crystallinity which are derived from abundant biobased polymers. ${ }^{73}$ Hydrogels from polysaccharide nanocrystals can be prepared directly from polysaccharide nanocrystals, such as cellulose nanocrystals and chitin nanocrystals, or 
polysaccharide nanocrystals composites with other matrices. Considering their unique physical and chemical properties, polysaccharide nanocrystals not only serve as reinforce nanofillers, but also endow hydrogels with other extraordinary performances. Here, we mainly introduce the preparation and their extraordinary properties of cellulose nanocrystals, chitin nanocrystals and starch nanocrystals, as well as their contributions in hydrogels functionalization.

\subsubsection{Cellulose nanocrystals (CNCs) and their applications in hydrogels}

Cellulose is a linear natural polymer consisting of 1,4-anhydro-D-glucopyranose units, which is the most abundant renewable organic material in the world. ${ }^{74} \mathrm{CNCs}$, the crystalline regions of cellulose, are extracted from wood, cotton and other cellulosic materials (Figure 5a) ${ }^{75}$ With different preparation methods, CNCs are synthesized with various functional groups on the surface, such as carboxyl groups, hydroxyl groups, aldehyde groups, and sulfate groups, which not only promote the dispersity of CNCs in water, but also offer the possibility for various chemical modifications on CNCs (Figure 5b).

CNCs are rigid rod-like nanocrystals with high crystallinity, large aspect ratio, and low density. They are only a few nanometers in width, but the length is distributed over a broad range, from tens of nanometers to several micrometers (Figure 5c). The high anisotropy and crystallinity of CNCs lead to promising mechanical properties with high axial stiffness up to $150 \mathrm{GPa}$ and high tensile strength in the range of 7.5-7.7 GPa. The axial Young's modulus of CNCs are comparable to those of Kevlar and steel. ${ }^{76}$

In addition to their extraordinary physical and surface chemical properties, CNCs possess unique liquid crystal behavior, which attracts growing interest in optical materials. On one hand, under suitable conditions, rod-like CNCs can spontaneously self-assemble into a chiral nematic structure, also called cholesteric structure. ${ }^{77}$ Within this structure, CNCs were aligned in parallel on a plane, then the planes assemble layer by layer in parallel with certain rotating angles, forming the helically twisted structure with certain helical pitch. ${ }^{78}$ This alignment is concentration-dependent arising from the balance between the rotational and translational entropies of individual CNCs nanorods (Figure 5d) ${ }^{79}$ This assembled chiral nematic structure 
would appear iridescent due to the diffraction of the incident light when the helical pitch of the structure is in the range of the wavelength of visible light. On the other hand, similar to molecular liquid crystals, the polarization of transmitted light would be changed by the alignment of CNCs owing to the phase difference, which can lead to great changes in interference colors when observed between crossed or parallel polarizers. ${ }^{80} \mathrm{CNCs}$ are birefringent nanoparticles originating from their highly crystalline structure with tightly aligned cellulose chains inside each CNCs spindle. Due to this unique rod-like morphology, the reflection index differed in parallel and perpendicular to the crystal axis, which induced the birefringence (Figure 5e). ${ }^{81}$ These special optical properties provide CNCs the ability to construct optical material with various colors.

Over the last decade, CNCs have attracted great attention in functional materials owing to their unique characteristics. Especially, $\mathrm{CNCs}$ have received growing interests in preparing functional hydrogels. In recent years, various functional CNCs composite hydrogels were constructed taking advantage of the high surface area, high mechanical strength, chemical active surface, excellent biocompatibility and other unique physical and chemical properties of CNCs. 

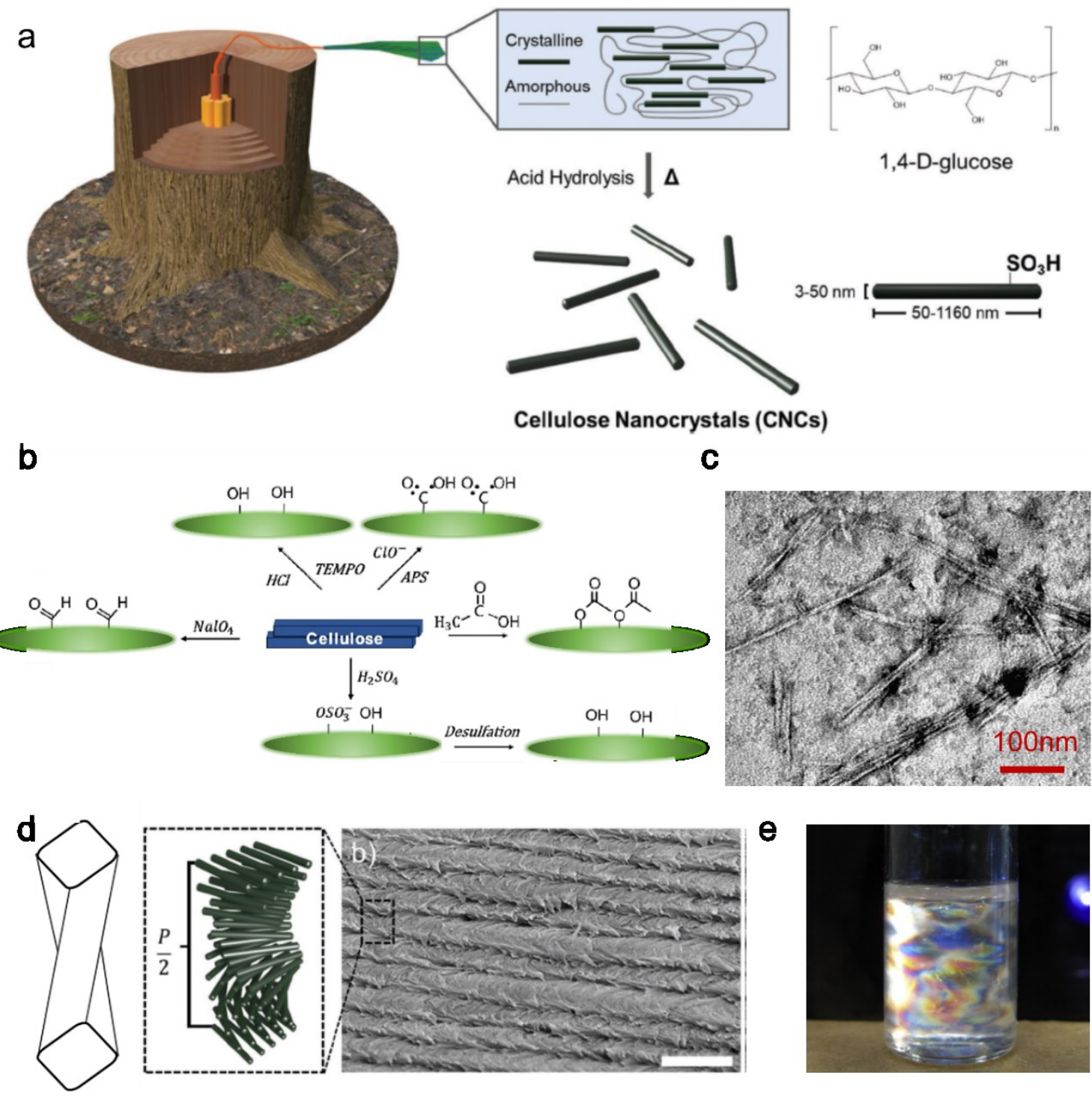

Figure 5. Isolation and unique properties of CNCs. (a) Isolation of CNCs by acid hydrolysis. (Reproduced from TRAN et al. ${ }^{75}$ Copyright from WILEY-VCH 2020) (b) Facile surface modification for CNCs. (c) TEM image of CNCs. (d) Chiral nematic structure of CNCs. (Reproduced from TRAN et al. ${ }^{75}$ Copyright from WILEY-VCH 2020) (e) CNCs suspension in water observed between crossed polarizers. (Reproduced from SIQUEIRA et al. ${ }^{81}$ Copyright from Springer 2010)

Hydrogels with CNCs. Most synthetic hydrogels are mechanically fragile, weak, and brittle, which extremely limits their practical applications. The high aspect ratio and stiffness of CNCs contribute to the formation of nanocomposite hydrogels with improved mechanical properties, due to the stress transfer from flexible polymer network to the stiff CNCs. Yang et al. reported 
the PEG hydrogels reinforced by CNCs displayed enhanced fracture stress, modulus and stretchability than pristine PEG hydrogels. The rearrangement of hydrogen bonding between CNCs and PEG provided the unique energy dissipation, which gives nanocomposite hydrogels the toughness and extensibility (Figure 6a). ${ }^{82}$ Stiffer and highly elastic CNCs crosslinked composite hydrogels were prepared by the introduction of polymerizable acrylate groups or polymer chains. The modified CNCs can act as multi-functional crosslinkers in hydrogels to improve the mechanical performance. Wang and coworkers reported a multi-responsive bilayer hydrogel actuator with the addition of methyl acrylamide modified CNCs to the cooperating layer. The weight-lifting capability for this hydrogel actuator designed gripper was highly improved to 18 times the weight of their own polymer weight. ${ }^{83}$ Besides the covalent bonds, diverse physical interactions were reported to connect CNCs with hydrogel network, such as ionic bonds, ${ }^{84}$ host-guest interactions, ${ }^{85}$ electrostatic attractions, ${ }^{86}$ which built recoverable energy dissipation system within hydrogels via the reversible dissociation and reassociation.

More importantly, diverse functional CNCs could be prepared due to facile surface modifications, which provides CNCs composite hydrogels with other promising functions and applications. Oechsle and coworkers reported a carbon dioxide $\left(\mathrm{CO}_{2}\right)$ switchable $\mathrm{CNCs}$ hydrogel, which was fabricated by mixing CNCs suspension with imidazole (Figure 6b). ${ }^{87}$ Through sparging $\mathrm{CO}_{2}, \mathrm{CNCs}$ were gelated, due to the electrostatic attractions between $\mathrm{CNCs}$ and protonated imidazole at low $\mathrm{pH}$. This process was reversible by simply sparging with nitrogen $\left(\mathrm{N}_{2}\right)$, leading to the deprotonation of imidazole and the subsequent electrostatic repulsion between them. Other stimuli-responsive polymers were also incorporated onto CNCs and subsequently transferred to hydrogels. For example, Zubik and coworkers reported the CNCs-g-PNIPAm temperature-responsive hydrogel, showing a potential application in wound dressing. ${ }^{88}$ Increasing the addition of CNCs to the system resulted in improved mechanical stability, while clear thermo-responsive behavior remained. The antibiotic and anti-protozoal compounds were loaded into the hydrogel at room temperature and then released at $37{ }^{\circ} \mathrm{C}$, which is suitable for wound dressing.

In addition, cellulose nanocrystals gained tremendous interest in fabricating photonic materials 
due to their self-assembly behavior. The self-assembled chiral nematic structures were constructed in various matrices to give various colors, including hydrogels. In particular, hydrogels show volume change in response to variations of osmotic pressure. Consequently, the volume change would lead to helical pitch change, which could generate tunable structural color. Hydrogels with stimuli-responsive photonic characteristic are of great significance for practical applications. Kelly et al. demonstrated a straightforward approach to prepare photonic hydrogels with long-range chiral nematic structure, which showed color changes in response to solvent, $\mathrm{pH}$ and temperature with their reversible swelling behavior in various media (Figure 6c). ${ }^{89}$ However, it's still a challenge to construct this chiral nematic structure within hydrogels, because the structure can be easily disrupted during the formation of hydrogels.

Besides, based on the birefringence of $\mathrm{CNCs}$, great efforts have been devoted to fabricating hydrogels with anisotropically aligned CNCs. Due to the collectively anisotropic alignment of CNCs, strong local birefringence emerged with light retardation, hydrogels with unidirectional aligned CNCs appear iridescence when viewed between crossed/parallel polarizers. Hiratani et al. reported stable and sensitive stimuli-responsive anisotropic hydrogels for sensing ionic strength and pressure, which was implemented by mechanical shearing aligned CNCs. ${ }^{90}$ Similar to this, various methods were developed to align CNCs via shearing force, such as 3D printing, capillary action, which were able to fix the alignment of CNCs in hydrogel network by the liquid to solid transition, known as gelation process. In particular, due to the unique solid and liquid nature of hydrogel, Huang et al. demonstrated a liquid behavior assisted fabrication of multidimensional birefringent materials from dynamic CNCs hybrid hydrogels. In this work, they utilized the mechanical stretching driven shear-thinning behavior in the highly dynamic hybrid hydrogels to align CNCs and preserved this alignment in the fast-relaxed polymer networks. Upon sequentially air-drying process, the surface tension further enhanced alignment of CNCs and achieved highly tunable birefringence (Figure 6d). ${ }^{91}$ 


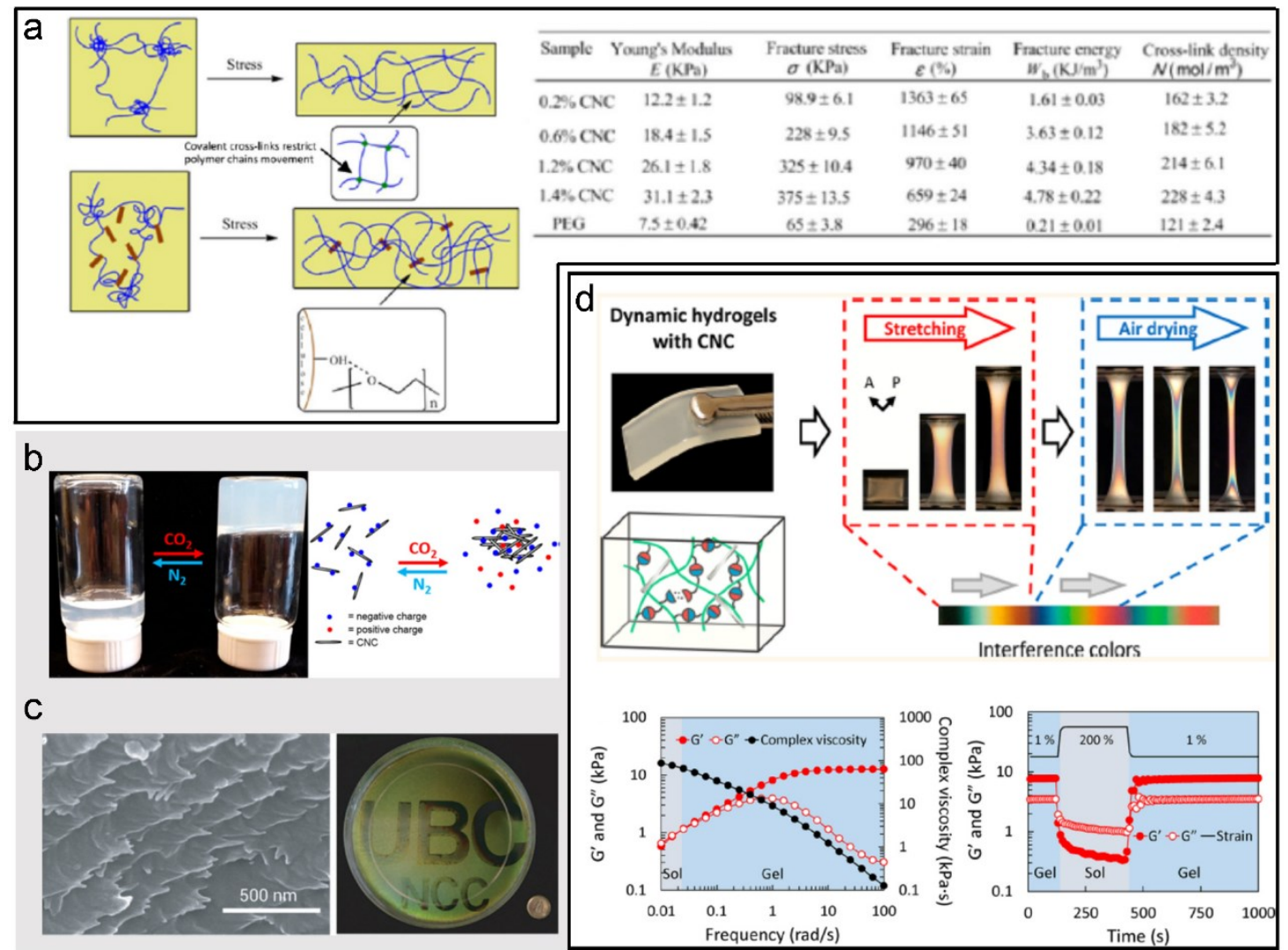

Figure 6. CNCs composite hydrogels. (a) PEG hydrogels reinforced by CNCs. (Reproduced from YANG et al. ${ }^{83}$ Copyright from American Chemical Society 2013) (b) $\mathrm{CO}_{2}$ responsive CNCs. (Reproduced from OECHSLE et al. ${ }^{87}$ Copyright from American Chemical Society 2018) (c) Stimuli-responsive photonic hydrogels. (Reproduced from KELLY et al. ${ }^{89}$ Copyright from WILEY-VCH 2013) (d) Liquid behavior assisted fabrication of birefringent materials from dynamic CNCs hybrid hydrogels. (Reproduced from HUANG et al. ${ }^{91}$ Copyright from American Chemical Society 2019)

\subsubsection{Chitin nanocrystals (ChNCs) and their applications in hydrogels}

Chitin is the second abundant semicrystalline biopolymer after cellulose, which is mainly synthesized by the biosynthetic method through a large number of living organisms such as shrimp, crab, tortoise, and insects (Figure 7a). ${ }^{92}$ Chitin is a linear polysaccharide with high molecular weight consisting of copolymer repeated units of $\beta$-(1 $\rightarrow 4)$-2-acetamido-2-deoxy- $\beta$ D-glucose and $\beta$ - $(1 \rightarrow 4)$-2-amino-2-deoxy- $\beta$-D-glucose. ${ }^{93}$ Apart from the acetyl amide group at C2 position, chitin shows a similar structure with cellulose. Chitin also has ordered and non- 
ordered regions. Similar to $\mathrm{CNCs}$, ChNCs were generated by removing the non-ordered regions of chitin under certain conditions. However, distinguished from $\mathrm{CNCs}$, $\mathrm{ChNCs}$ were produced from biological tissues which combined with some impurities including protein and other organic compounds. Hence, chitin has a pretreatment with demineralization, deproteinization, and decoloration processes to remove these impurities before isolation (Figure 7b) $)^{93,94}$.

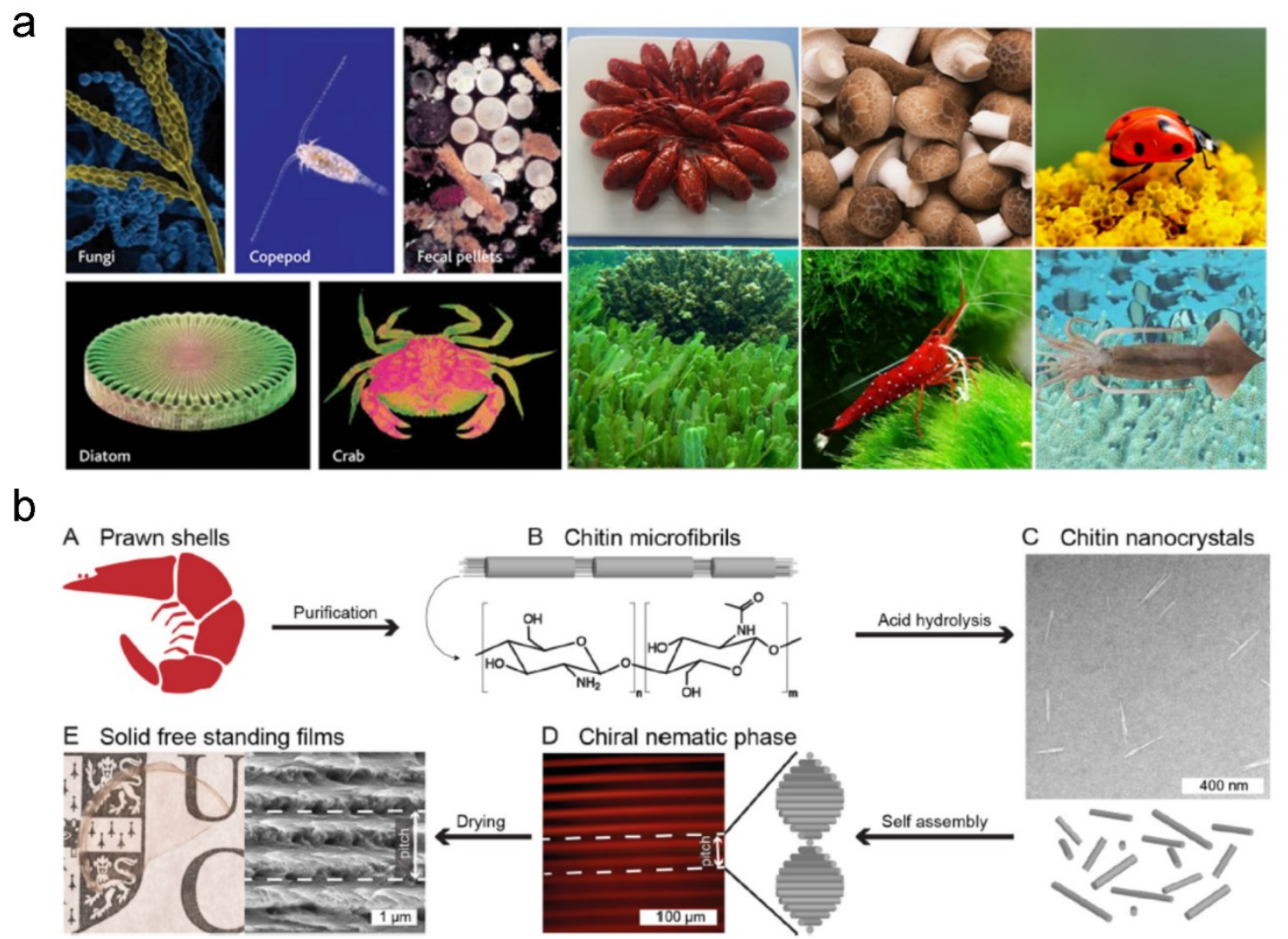

Figure 7. (a) Abundant sources of ChNCs. (Reproduced from DUAN et al. ${ }^{92}$ Copyright from Elsevier 2018) (b) ChNCs extracted from acid hydrolysis and their properties. (Reproduced from NARKEVICIUS et al. ${ }^{94}$ Copyright from American Chemical Society 2019)

ChNCs possess similar physical and chemical properties with CNCs. ChNCs also display rodlike or spindle-like morphology, their dimensions and crystallinities are determined by their sources and preparation methods. In general, ChNCs synthesized from diverse sources are roughly 4-80 $\mathrm{nm}$ in width and has 50-10000 $\mathrm{nm}$ in length. ${ }^{95} \mathrm{ChNCs}$ have high strength modulus, which is up to $150 \mathrm{GPa}$ for longitudinal modulus and nearly $15 \mathrm{GPa}$ for the transverse modulus. ${ }^{93}$ ChNCs have abundant hydroxyl groups on the surface which provide the possibility 
for various modifications to introduce specific functions and to expand their applications.

ChNCs also possess extraordinary optical properties, arising from the self-assembled chiral nematic structure and birefringence. ${ }^{96}$ In water, ChNCs are positively charged, due to the protonated amino groups at the surface $(\mathrm{pKa} \sim 6.3)$. When in acidic aqueous solution ( $\mathrm{pH}$ below 6.3), ChNCs are stable, and exhibit liquid crystalline behavior above critical concentration. ${ }^{94}$ Similar to CNCs, this is an entropy driven process dominated by their anisotropic shapes and the mutual interactions between the charged rod-like ChNCs.

Hydrogels with ChNCs. ChNCs are widely used as reinforcing additives in functional hydrogels. Nanocomposite hydrogels with ChNCs show improved toughness and stretchability, similar to CNCs, due to the load transfer from flexible polymer chains to rigid ChNCs and more easily entangled fibrous structure. ${ }^{97,}{ }^{98}$ In addition to mechanical reinforcement, $\mathrm{ChNCs}$ also possess excellent biocompatibility and low cytotoxicity, Huang and coworkers prepared $\mathrm{ChNCs} /$ alginate nanocomposite hydrogel by the $\mathrm{pH}$-induced charge shifting behavior of ChNCs, which largely improved the mechanical behaviors. These nanocomposite hydrogels were applied as bone scaffolds for osteoblast cells and revealed that the addition of ChNCs noticeably promoted cell adhesion and proliferation (Figure 8) ${ }^{99}$ Besides, the deacetylated ChNCs have natural antimicrobial property due to the polycationic nature, which makes ChNCs promising nanomaterials in wound dressing. ${ }^{100}$

Similar to CNCs, ChNCs are promising in designing optical materials based on this selfassembly behavior. Using $\mathrm{ChNCs}$ to fabricate structured color hydrogels is rarely reported, but pioneer work has been published in controlling their assembly and alignment in polymer membranes. Narkevicius and co-workers systematically studied how the properties of chitin nanocrystal suspensions influence the self-assembly behavior. ${ }^{94}$ The optimized ChNCs could prepare chiral nematic $\mathrm{ChNCs}$ films with tunable helical pitches by varying the ionic strength and $\mathrm{pH}$ of the suspensions. Besides, $\mathrm{ChNCs}$ are also birefringent nanoparticles originating from their highly crystalline structure and the rod-like morphology, the reflection index differed in parallel and perpendicular to the crystal axis, which induced the birefringence. Anisotropically aligned ChNCs showed vivid interference colors due to the birefringence. ${ }^{101}$ These 
extraordinary photonic properties can be expected in designing optical hydrogels in the future.
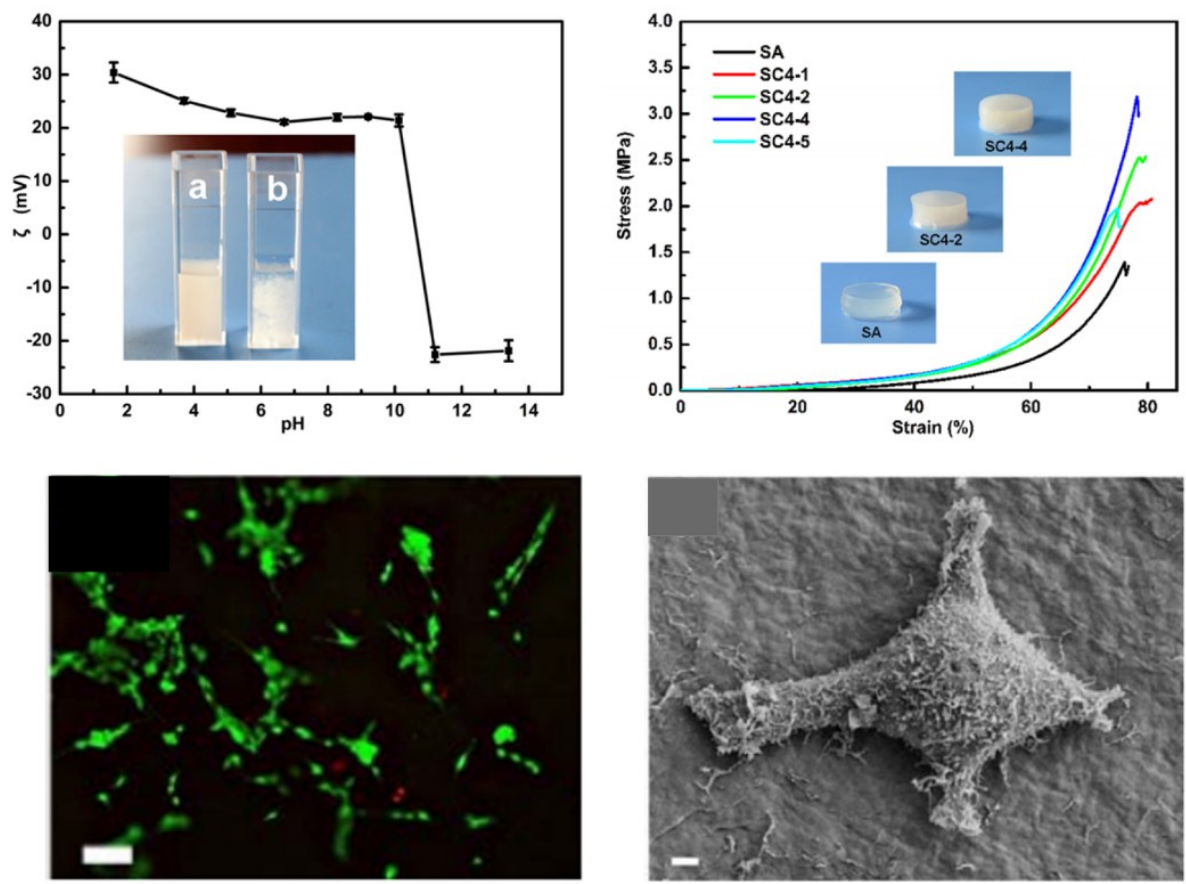

Figure 8. ChNCs/alginate nanocomposite hydrogel. (Reproduced from HUANG et al. ${ }^{99}$ Copyright from American Chemical Society 2015)

\subsubsection{Starch nanocrystals (SNCs) and their applications in hydrogels}

Starch is an abundant natural polymer, produced by various plants as a source of stored energy. ${ }^{102}$ The starch structure is multi-scale and lies in the (i) starch granule $(2-100 \mu \mathrm{m})$, where displays as (ii) growth rings (120-500 nm). The growth rings are comprised of (iv) blocklets (20-50 nm) with (iii) amorphous and crystalline lamellae $(9 \mathrm{~nm})$ containing (vii) amylopectin and (viii) amylose chains $(0.1-1 \mathrm{~nm})$, as presented in Figure 9a. ${ }^{103-105}$ All starches are constituted by $\alpha$-D-glucopyranose units in linear chains linked by $\alpha(1 \rightarrow 4)$ bonds in amylose and in branched chains linked by $\alpha(1 \rightarrow 6)$ bonds from the linear chains in amylopectin. They are ordered in alternating crystalline and amorphous lamellae $(9 \mathrm{~nm})$ in the 2-100 $\mu \mathrm{m}$ starch granules.

SNCs are generated by acid hydrolysis, enzymatic hydrolysis, where the disordered or less ordered parts of starch granules are preferentially hydrolyzed, the crystalline regions remain. ${ }^{106}$ Distinguished from CNCs and ChNCs, SNCs are crystalline platelets. The structure and 
morphology of the isolated starch nanocrystals commonly depend on the source of starch with various crystallization type, differed relative proportion of crystalline amylose and amylopectin. ${ }^{107}$ The size and yield of SNCs are influenced by the hydrolysis conditions (such as acid type, acid concentration, temperature, and time).
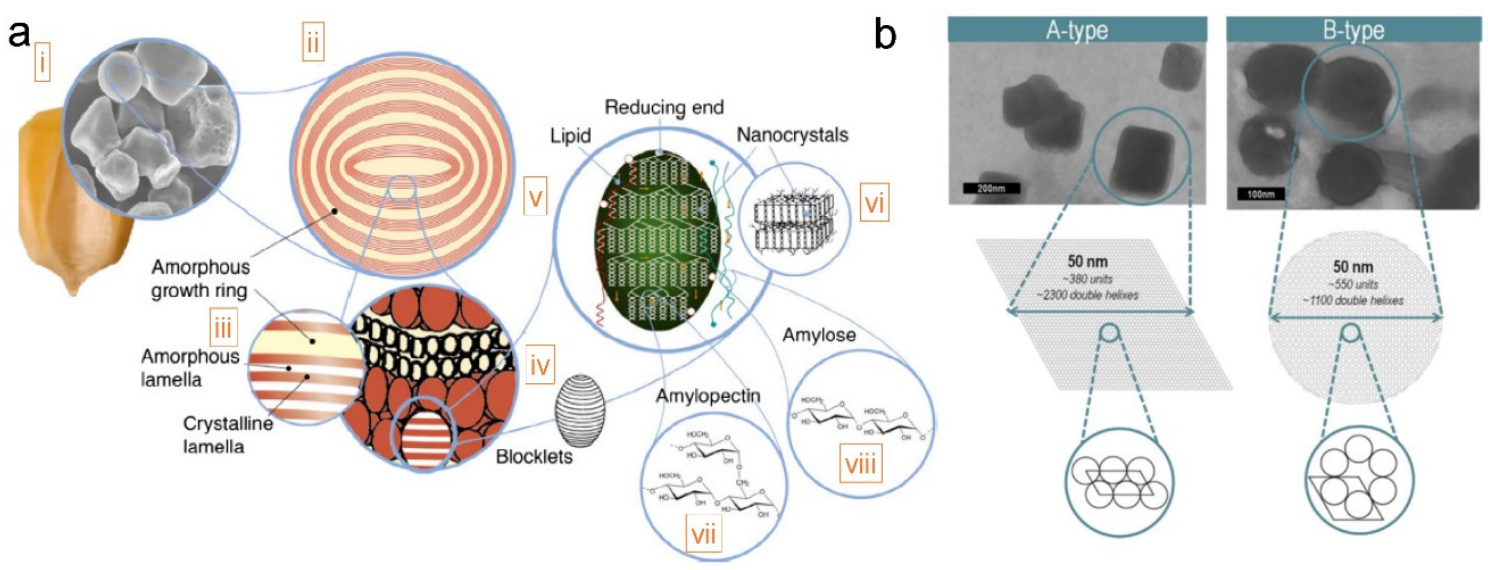

Figure 9. Structure of starch and the SNCs extracted from A-crystalline type and B-crystalline type starch granules. (a) Concentric multiscale structure of starch. (i) Typical starch granules from normal maize $(30 \mu \mathrm{m})$, (ii) Amorphous and semicrystalline growth rings (120-500 nm), (iii) amorphous and crystalline lamellae (9 nm), (iv) magnified details of the semi-crystalline growth ring with blocklets $(20-50 \mathrm{~nm})$, constituting unit of the growth rings, (v) amylopectin double helices forming the crystalline lamellae of the blocklets, (vi) nanocrystals: other representation of the crystalline lamellae called SNC when separated by acid hydrolysis, (vii) amylopectin's molecular structure, (viii) amylose's molecular structure $(0.1-1 \mathrm{~nm})$. (Reproduced from TANG et al. ${ }^{103}$ Copyright from Elsevier 2006; DONALD et al. ${ }^{104}$ Copyright from Science and Technology Facilities Council 1997; GALLANT et al. ${ }^{105}$ Copyright from Elsevier 1997) (b) SNCs extracted from different starch granules of waxy maize starch (A-type) and high amylose starch (B-type). (Reproduced from LECORRE et al. ${ }^{107}$ Copyright from Springer 2011)

In recent years, chemical modifications were performed on their hydroxyl groups of SNCs, such as chemical reaction with small molecules, polymer chains grafting onto the surface with coupling agents, polymer chains growing on the surface with polymerization of monomers. ${ }^{108}$ Those surface modifications ensure SNCs compatible with many polymer matrices. 

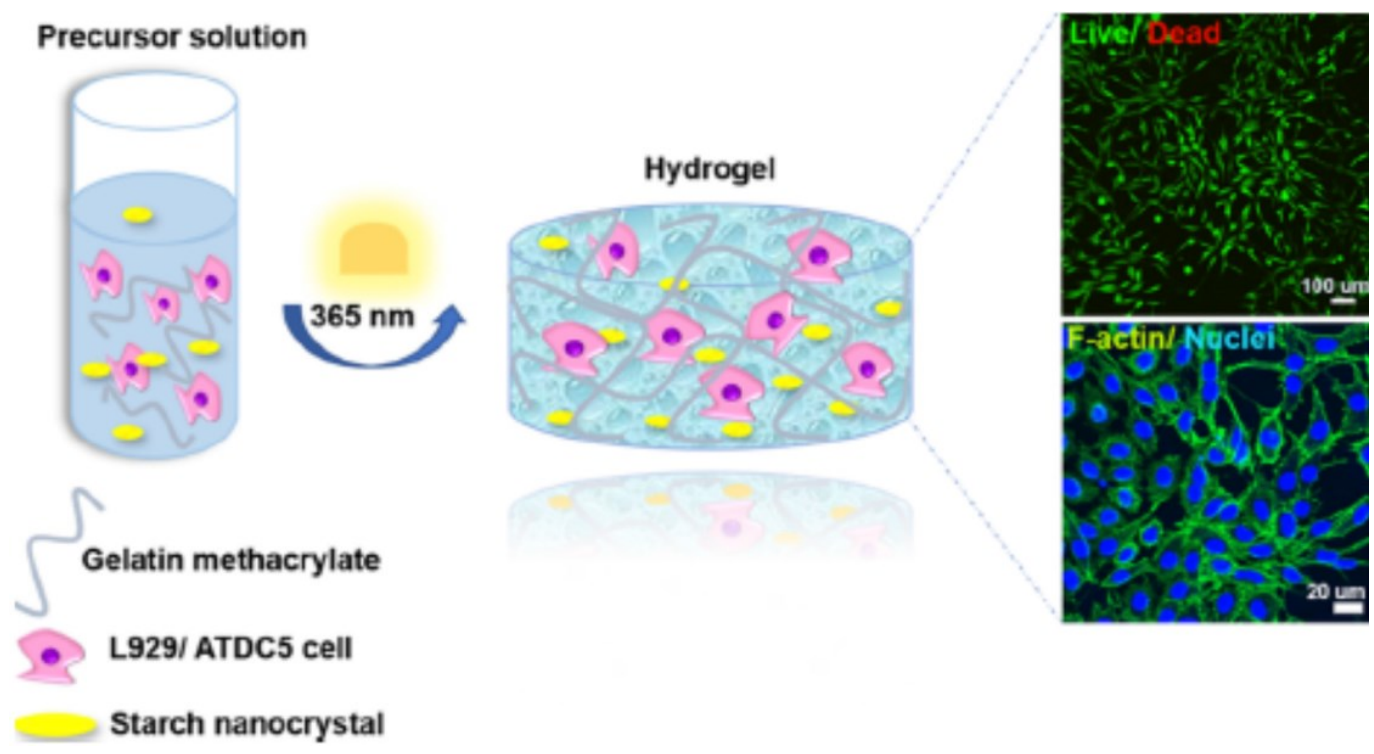

Figure 10. SNCs composite hydrogel as cell-instructive materials. (Reproduced from PILUSO et al. ${ }^{110}$ Copyright from American Chemical Society 2019)

Hydrogels with SNCs. SNCs in hydrogels were normally used as mechanical reinforcement fillers with similar improvement mechanism with CNCs and ChNCs. Owing to the unique excellent biocompatibility, low toxicity, biodegradability, they are promising materials in drug carriers, tissue engineering, and skin adhesives. ${ }^{109}$ Piluso et al. developed 3D microenvironments with starch nanocrystals embedding in the gelatin matrix as cell-instructive materials. The incorporation of SNCs led to improved compressive modulus, at the same time, composite hydrogel showed potential usage for cartilage tissue engineering with the evidence that the chondrogenic progenitor ATDC5 cells maintained viability around 90\% but displayed a round morphology, especially in the hydrogels with SNCs (Figure 10). ${ }^{110}$

Overall, applying polysaccharide nanocrystals to construct functional hydrogels attracts growing interests both in academia and industry. Within the scope of polysaccharide nanocrystals, CNCs still hold great promise in functionalizing hydrogels in light of their mature synthesis technology with specific surface groups on demand, rigid rod-like morphology and their extraordinary liquid crystal nature. 


\section{Objective of the study}

Hydrogels are crosslinked 3D polymer networks with amounts of water in their highly porous structure. The crosslinked polymer networks endow hydrogels with the properties of soft and elastic solid, while the high-water content in hydrogels gives rise to liquid-like properties, such as good permeability to various chemicals, plastic behavior and adjustable optical characteristics. In addition, hydrogels have some unique properties, such as the responsiveness and swelling, brought by their tailorable polymer network and exchangeable aqueous system. All these features make hydrogels promising semi/solid materials with diverse practical applications.

Great efforts have been made to hydrogel functionalization, especially, functionalization by diverse nanocomposites, to satisfy various applications. As aforementioned in the introduction section, CNCs composite hydrogels have great potential as functional materials owing to their bio-based and renewable nature, excellent mechanical properties, numerous chemical-active groups on the surface, and unique optical properties. However, the CNCs applied in functional hydrogels and CNCs assistance in functional hydrogel fabrication, as well as the transferring of unique optical properties from CNCs to hydrogels and dry materials still need further research.

Therefore, the present study aims to develop functional composite hydrogels based on CNCs. The main objective covers the following points:

1. To prepare crosslinkable CNCs with surface-attached methyl acrylamide groups, and to use them as reinforcement nanocrosslinkers in the hydrogel actuators. (Publication 1)

2. To prepare closed hollow hydrogels with distinctively tunable inner and outer walls through the assistance of CNCs to improve spatial crosslinking distinction. (Publication 2)

3. To transfer and integrate the birefringence of CNCs and surface plasmon resonance of gold nanorods in fabricating optical polymer films based on the thixotropy of dynamic hydrogels.

(Publication 3) 


\section{Results and discussion}

\subsection{Bilayer hydrogel actuators with programmable and precisely tunable motions}

As reported in Publication 1, a novel type of bilayer hydrogel actuators (BHAs) was prepared comprising of a poly(N-isopropylacrylamide) (PNIPAm) and a poly(N-hydroxyethyl acrylamide) (PHEAm) hydrogel layer with various compositions. Cellulose nanocrystals (CNCs) are water dispersible with a modifiable surface. At first, we prepared methyl acrylamide groups modified CNCs (CNCs-MAm) (Figure 11).

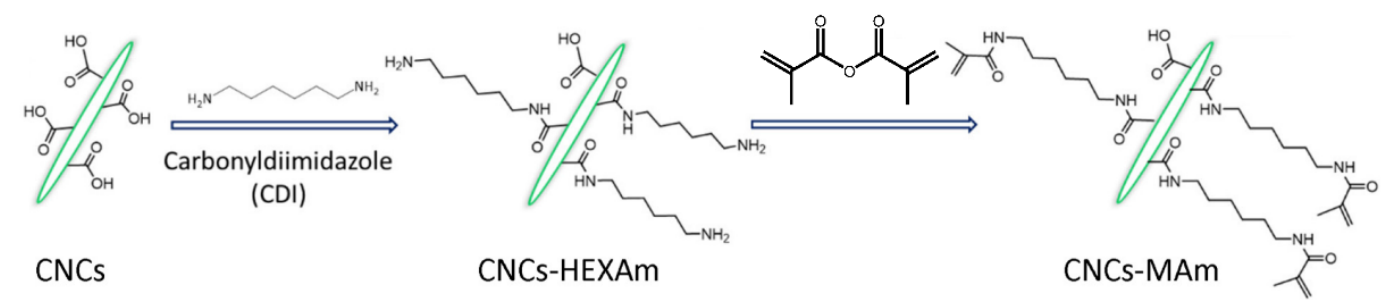

Figure 11. Surface modifications of CNCs.

Then, as shown in Figure 12a, BHAs were prepared via a two-step method. The first layer was chemically crosslinked PNIPAm hydrogels as the active layer. Then, various PHEAm layers were fabricated above the PNIPAm layer as the cooperating layers. BHAs were obtained after equilibrated in deionized water. PHEAm hydrogels in diverse BHAs were prepared with three different compositions: bulk crosslinked PHEAm in BHAs/P, PHEAm networks containing reinforcing $\mathrm{CNCs}$ (PHEAm/CNCs) in $\mathrm{BHAs} / \mathrm{CNCs}$, and PHEAm networks containing crosslinkable CNCs-MAm (PHEAm/CNCs-MAm) in BHAs/CNCs-MAm.

The scanning electron microscopy (SEM) images in Figure 12b clearly showed the distinguishable PHEAm/CNCs-MAm layer and PNIPAm layer with different pore topologies within BHAs after freeze-drying. A much denser area was visible at the interface of the two layers, indicating the interpenetration of two kinds of polymer chains in this interfacial area. This dense interface in turn enhanced the connection of the two hydrogel layers. As a result, the covalently crosslinked interactions tightly fix the two hydrogel layers together, which were sufficient to tolerate the swelling and actuation behaviors triggered by various stimuli. 
Furthermore, the introduction of CNCs and crosslinkable CNCs-MAm led to much smaller pore size for the cooperating hydrogel layers.

a

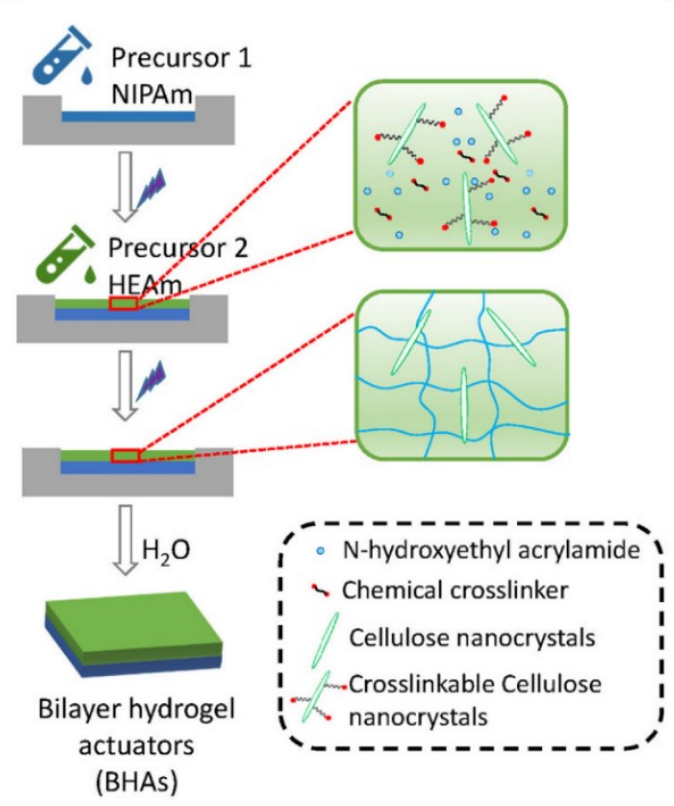

b

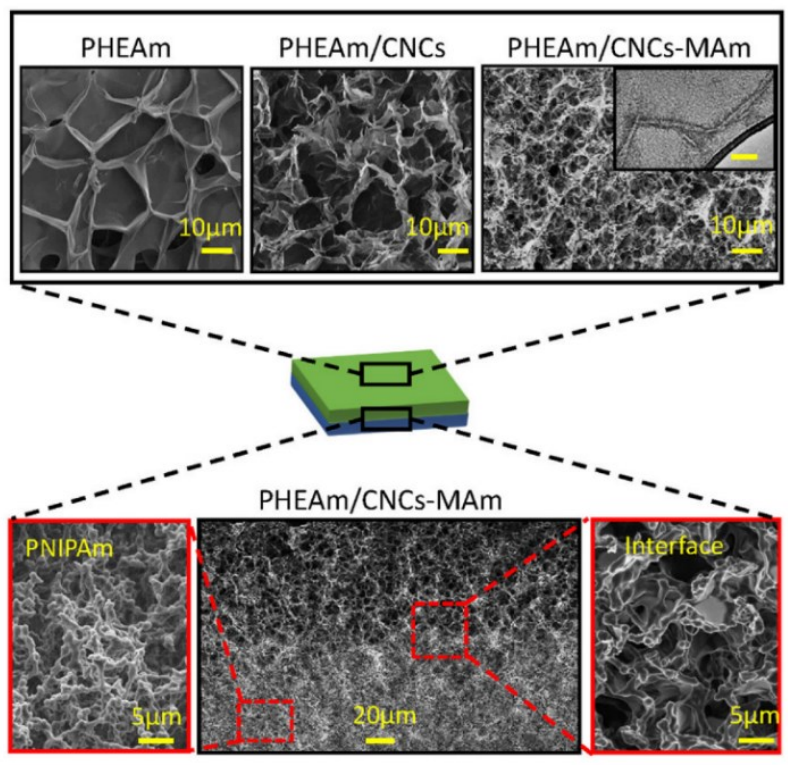

Figure 12. Schematic illustration for the preparation and the microstructures of BHAs. (TEM image of CNCs-MAm with the scale bar of $50 \mathrm{~nm}$ ).

The smaller pore size could be attributed to the extra crosslinking brought by the CNCs-MAm, which can be further proved by the smallest swelling ratio for PHEAm/CNCs-MAm comparing with its in PHEAm/CNCs and PHEAm/P (Figure 13a). The mechanical properties of various BHAs with distinct compositions were further characterized. In particular, the mechanical properties of cooperating PHEAm layers based on PHEAm with various compositions were characterized due to their distinct microstructures, while the active PNIPAm layers maintained equal in all BHAs. As exhibited in Figure 13b, the addition of CNCs and CNCs-MAm improved the stiffness and toughness of hydrogels with diverse extents. While CNCs only acted as neutral nanofillers and showed limited enhancement on the breaking stress of resulting hydrogels to about 2.5 times of PHEAm hydrogels, crosslinkable CNCs-MAm efficiently increased the breaking stress of obtained hydrogels to roughly 14 times of PHEAm hydrogels. Furthermore, the tensile tests clearly displayed the effective toughening by introducing CNCs and CNCs-MAm into the PHEAm hydrogels. 

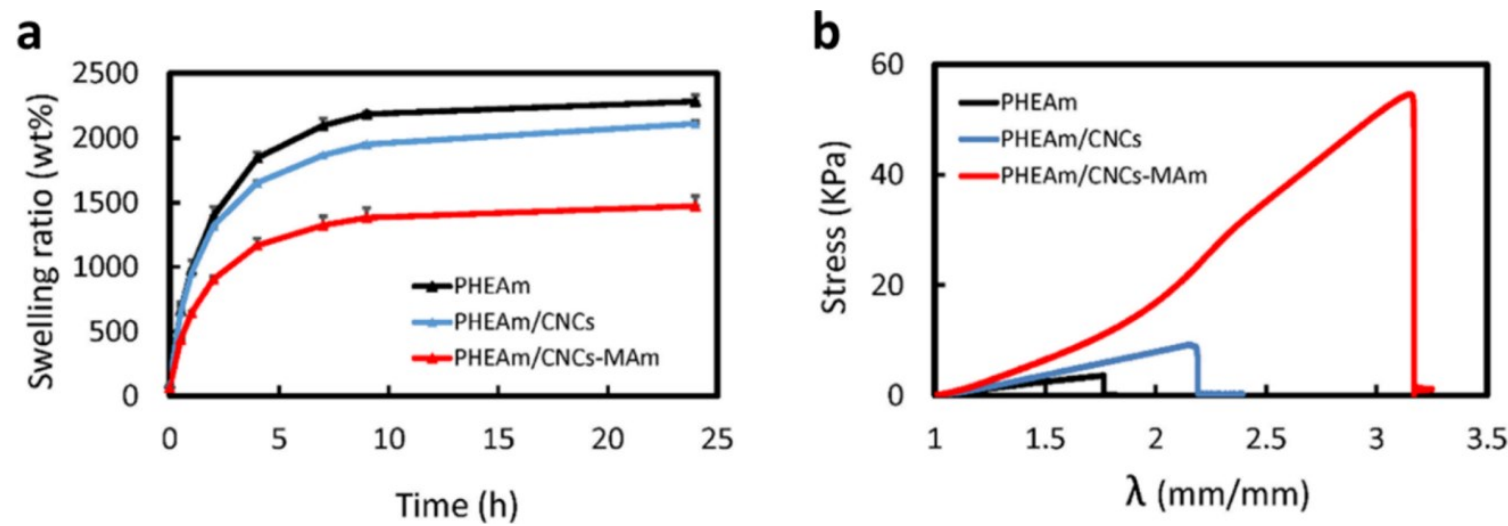

Figure 13. Swelling behaviors and mechanical properties of the cooperating layers

Considering the great difference in mechanical properties caused by the introduction of CNCs and CNCs-MAm in cooperating layers, we further studied the thermal behaviors of BHAs containing diverse cooperating layers. PNIPAm has a (Lower critical solution temperature) LCST at about $32{ }^{\circ} \mathrm{C}$ and exhibits greater hydrophobicity at the temperature above its LCST. When BHAs were immersed into water of $40{ }^{\circ} \mathrm{C}$, PNIPAm chains aggregated which induced shrinkage of the PNIPAm layers, all BHAs bended to the PNIPAm side (Figure 14a). The magnitudes of their dynamic bending motions were illustrated by the corresponding curvatures of BHAs after a certain time. Obviously, BHAs containing diverse compositions showed distinct bending amplitudes according to their curvatures after the same bending times during the dynamic process (Figure 14b). Generally, the continuous bending completed within 6 min and the shapes of the BHAs at 6 min were set as the final state. The BHAs/CNCs-MAm exhibited the smallest motion range and the curvature lay between $0.018-0.2$, while BHAs/P showed the largest bending range with the curvature roughly between 0.11-0.6. The curvatures of BHAs/CNCs during the bending changed from 0.12 to 0.47 . Because of the equal PNIPAm layers, this difference should be mainly attributed to the significant difference in the mechanical properties of the cooperating layers. The stiffer cooperating layer would sacrifice the bending amplitude for the BHAs. 
a

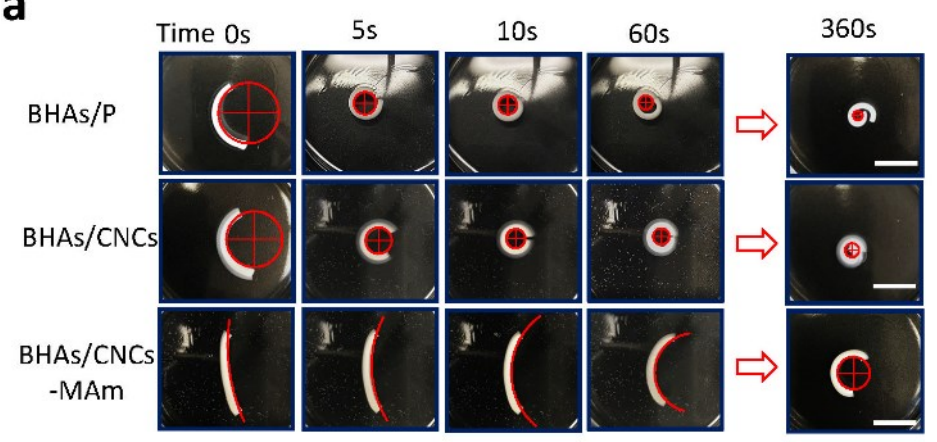

b

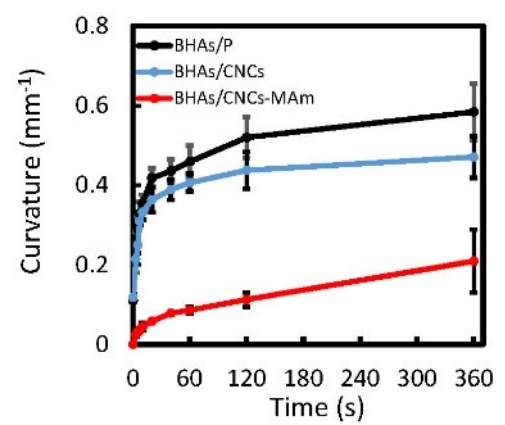

Figure 14. Dynamic thermal behaviors of BHAs in water of $40^{\circ} \mathrm{C}$ (scale bars: $1 \mathrm{~cm}$ ).

However, when the BHAs were designed as soft grippers to fulfill the grasping and releasing actions via varying the temperature of DI water (Figure 15a). With the reinforcement of the cooperating PHEAm layers with CNCs or CNCs-MAm, the grabbing capabilities of BHAs were largely increased (Figure 15b). BHAs/CNCs-MAm showed the highest maximum weight ratio and could lift items that were about 18 times the weight of own polymer weight. While the BHAs/CNCs could hold items of roughly $1400 \mathrm{wt} \%$ of own polymer weight, and BHAs containing PHEAm only could lift objects of $800 \mathrm{wt} \%$ of own polymer weight. Therefore, stronger cooperating layers highly promoted the loading capability of BHAs, independent on the active PNIPAm layers.
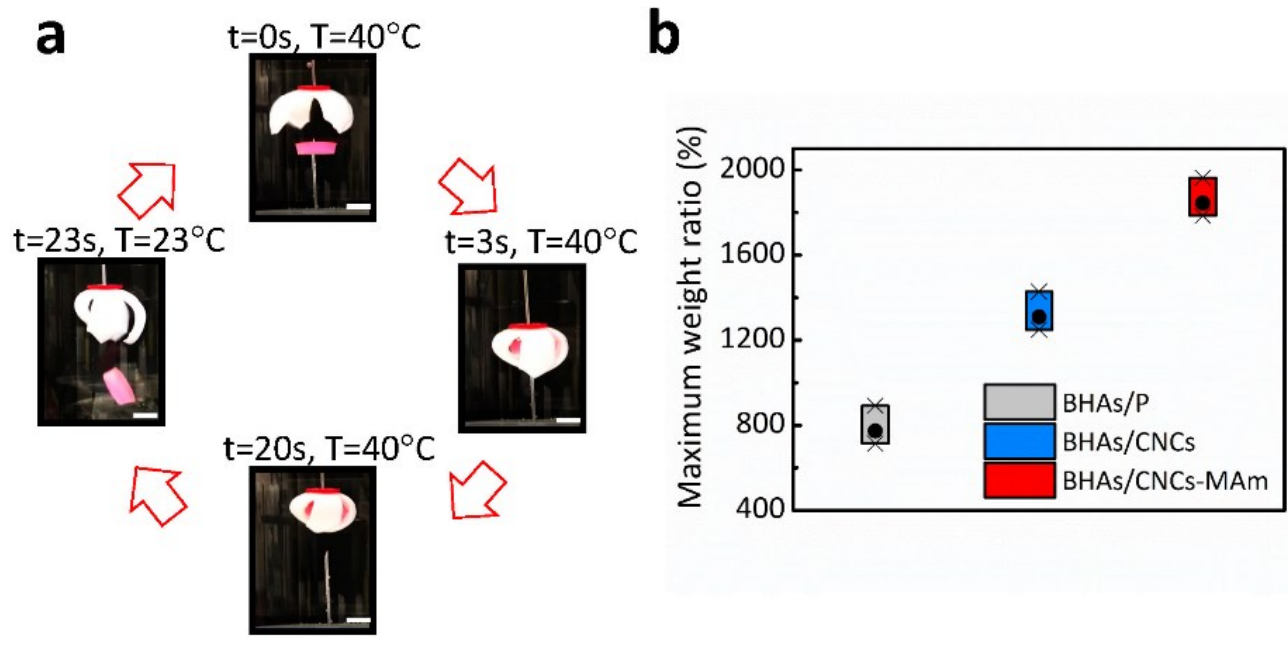

Figure 15. BHAs designed as gripper to grasp and release of target object (scale bars: $1 \mathrm{~cm}$ ). maximum weight ratios $=$ maximum weights that actuators can lift/own polymer weights. 
In addition, the thermal triggered actuator would deform until to its final state with uncontrollable intermediate state. These BHAs managed to bend with controllable motion amplitude which can be programmed to stop at a certain amplitude via their unique solventresponsive properties in ethanol/water mixtures. As shown in Figure 16a, the co-nonsolvency property of PNIPAm in ethanol/water mixtures endows PNIPAm hydrogels with the ability to swell and deswell depending on the ratio of both solvents. At the same time, various PHEAm cooperating layers shrink more strongly with increasing ethanol content (Figure 16b).
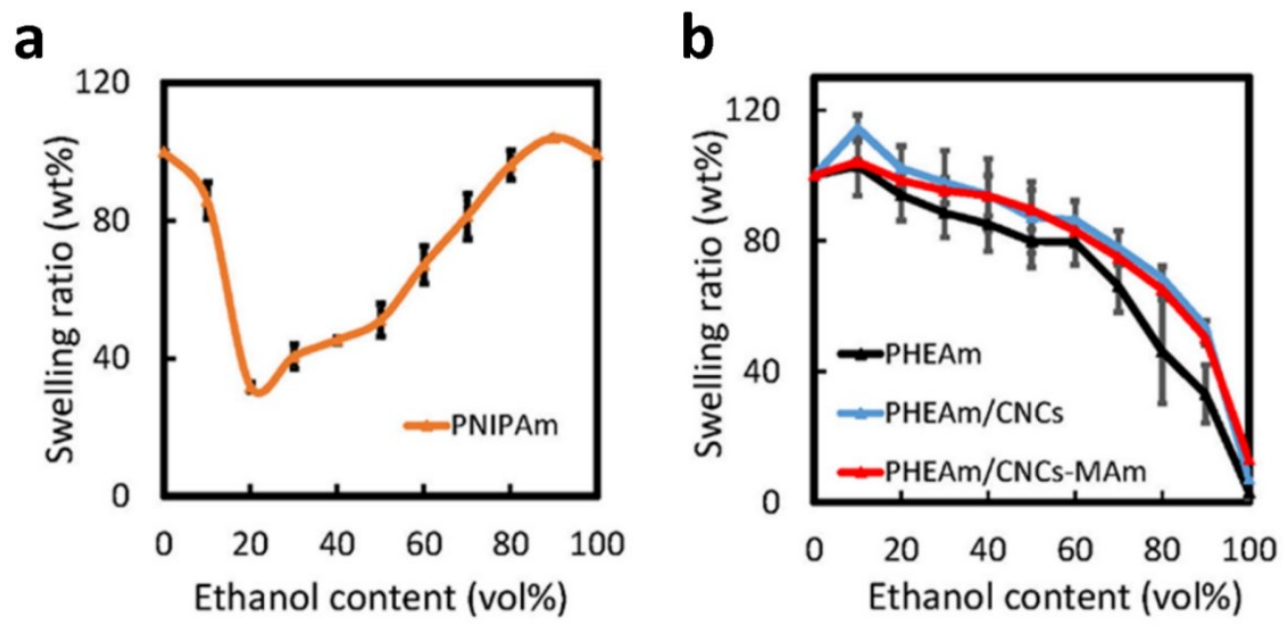

Figure 16. Swelling behaviors of PNIPAm layers (a) and various PHEAm layers (b) in diverse water/ethanol mixtures.

Nevertheless, these two different behaviors of the PNIPAm and PHEAm hydrogel layers in the ethanol/water mixtures synergistically contributed to extraordinary solvent-responsive behaviors of our BHAs. Subsequently, this co-solvent actuation not only generated bidirectional bending motions, but also the bending amplitudes (represented by their corresponding curvatures) could be adjusted by changing the solvent composition, afterwards, the BHAs could be fixed at a certain bending state (Figure 17a, b). It is obvious that the compositions and thus the mechanical properties of the cooperating PHEAm layers significantly affected the performance of these diverse BHAs (Figure 17a, b). Thus, this ethanol-triggered actuation allowed BHAs to autonomously change their bending direction and amplitudes according to the ethanol content in the surrounding environment. Moreover, artificial grippers based on such BHAs can be used in complex conditions, such as to transport 
items through gates (Figure 17c). As showed on the gripper of BHAs/CNCs, the gripper in their smallest size easily passed the narrow gate within the ethanol/water mixture with $20 \mathrm{vol} \%$ ethanol. By increasing the ethanol content to $80 \mathrm{vol} \%$, the gripper expanded and could grab a target object. By diluting the solvent to ethanol content of $20 \mathrm{vol} \%$, the gripper easily grabbed the object and took it through the narrow gate. Eventually, this object was released in another surrounding as designed, e.g. in pure water. Thus, such BHAs showed promising practical applications in diverse fields.

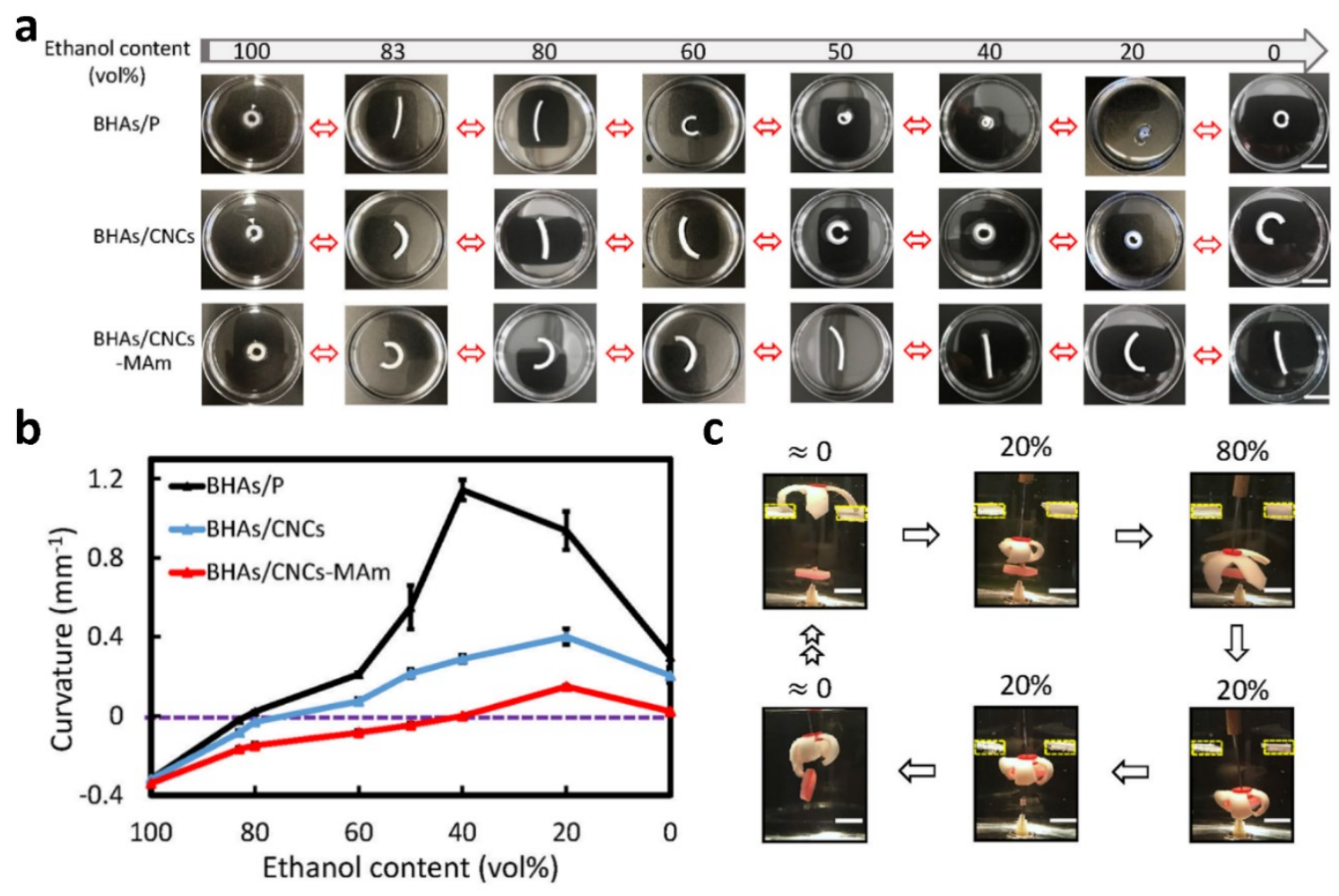

Figure 17. Dynamic behaviors of BHAs triggered by ethanol/water mixture. All BHAs were constituted by the PNIPAm layers on the right side and PHEAm layers on the left side (scale bars: $10 \mathrm{~mm})$.

In the present section, we reported a series of bilayer hydrogel actuators. They demonstrated advantageous controllable thermal-responsive and solvent-driven actuation performance. The incorporation of strengthening CNCs and CNCs-MAm in the cooperating PHEAm layers effectively improved the loading capacities of BHAs, although the they reduced motion amplitude. The ethanol driven actuation provides the feasibility to exactly tune the bending amplitude and bending direction of BHAs by adjusting the ethanol content. Thus, these pre- 
programmable motions and the feasibility to spontaneously freeze the state of BHAs demonstrate their great peculiarity.

\subsection{Temperature-Responsive, Manipulable Cavitary Hydrogel Containers by}

\section{Macroscopic Spatial Surface-Interior Separation}

Synthetic macroscopic materials transforming from bulk solid or semisolid to closed hollow structure with distinct outer and inner microstructures is rarely reported. In Publication 2, we demonstrated an in situ method for directing macroscopic spatial surface-interior separation from bulk dynamic hydrogels to closed 3D Janus hollow hydrogels via constructing competitively crosslinking gradient within dynamic hydrogels. The original crosslinking of phenylboronic acid/catechol complexes was disrupted and replaced by stronger crosslinking of $\mathrm{Fe}^{3+} /$ catechol associations, generating gradually weakened crosslinking from outside to inside. Sequential decomposition of weak crosslinking in the inner core within the densely crosslinking hydrogel shell, leading to closed hollow hydrogels with tunable dense outer shell and fluffy inner surface.
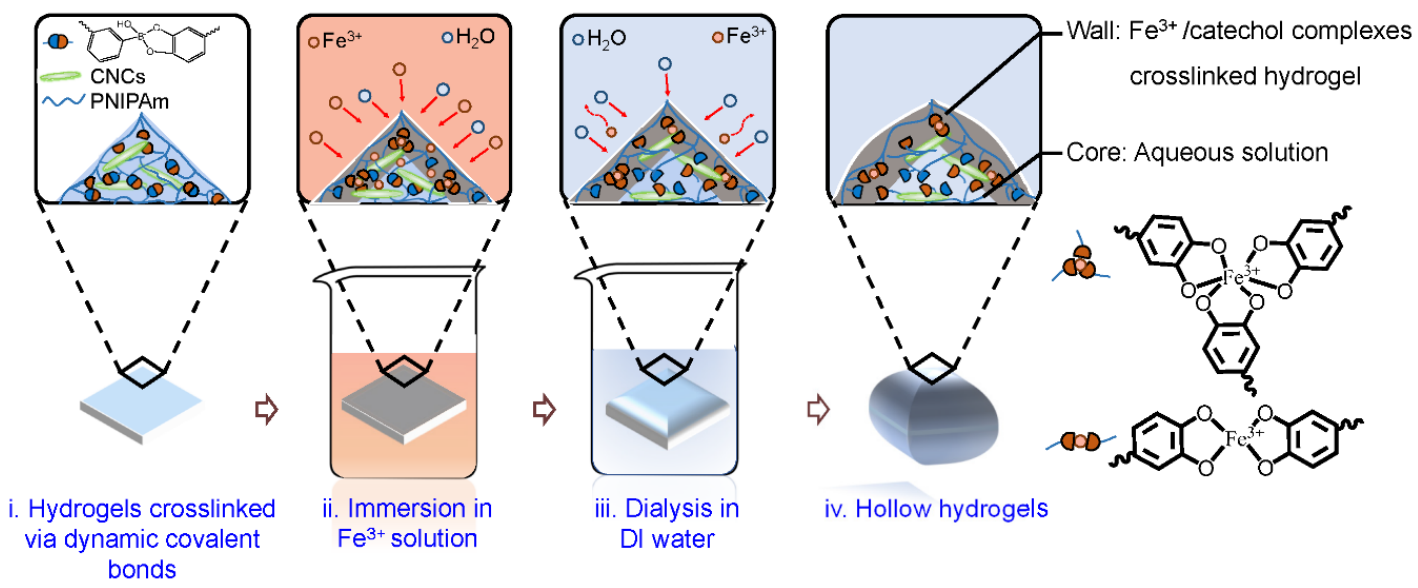

Figure 18. Schematic illustration for the preparation of closed hollow hydrogels.

In the present work, firstly, dynamic hydrogels were prepared comprising of PNIPAm hydrogel crosslinked by the dynamic covalent bonds of phenylboronic acid/catechol with the presence of CNCs in buffer solutions of $\mathrm{pH} 10$ (associate constant $\mathrm{Ka} \approx 0.919 \times 10^{3} \mathrm{M}^{-1}$ ) (Figure 18-i). Then, the dynamic hydrogels were immersed into ferric chloride solution $(0.1 \mathrm{M}$ in $\mathrm{pH} 10$ 
borate buffer solution) for controlled time, which allowed the $\mathrm{Fe}^{3+}$ to diffuse into hydrogels from the surrounding solution (Figure 18-ii). During this process, the $\mathrm{Fe}^{3+}$ ions formed complexes with catechol and replaced initial boronate ester bonds due to their much higher $\mathrm{Ka}$ $\left(10^{37}-10^{40} \mathrm{M}^{-1}\right)$. Along with the $\mathrm{Fe}^{3+}$ penetration, the $\mathrm{Fe}^{3+}$ concentration gradient resulted in gradually weakened $\mathrm{Fe}^{3+} /$ catechol crosslinking in the hydrogel network from surface to core. After soaking in $\mathrm{Fe}^{3+}$ solution with certain time, the treated hydrogels were transferred into DI water to dialyze until the weight was constant (Figure 18-iii). During this process, the hydrogel swelled largely driven by osmotic pressure, resulting in decomposition of weak crosslinking, but strong crosslinking can maintain. As a result, the outer part with strong crosslinking formed the stable hydrogel wall. At the same time, the inner part where the $\mathrm{Fe}^{3+}$ did not reach was dominated by boronate ester bonds crosslinked part dissociated into extremely loosely crosslinked polymer chains. As a result, the thin flat bulk hydrogels experienced a macroscopic spatial surface-interior separation process to generate 3D closed continuous hydrogels with enclosed solutions within hollow hydrogels (HHs/P represented polymeric hollow hydrogels without CNCs, and $\mathrm{HHs} / \mathrm{CNCs}$ with $\mathrm{CNCs}$ ) (Figure 18-iv).
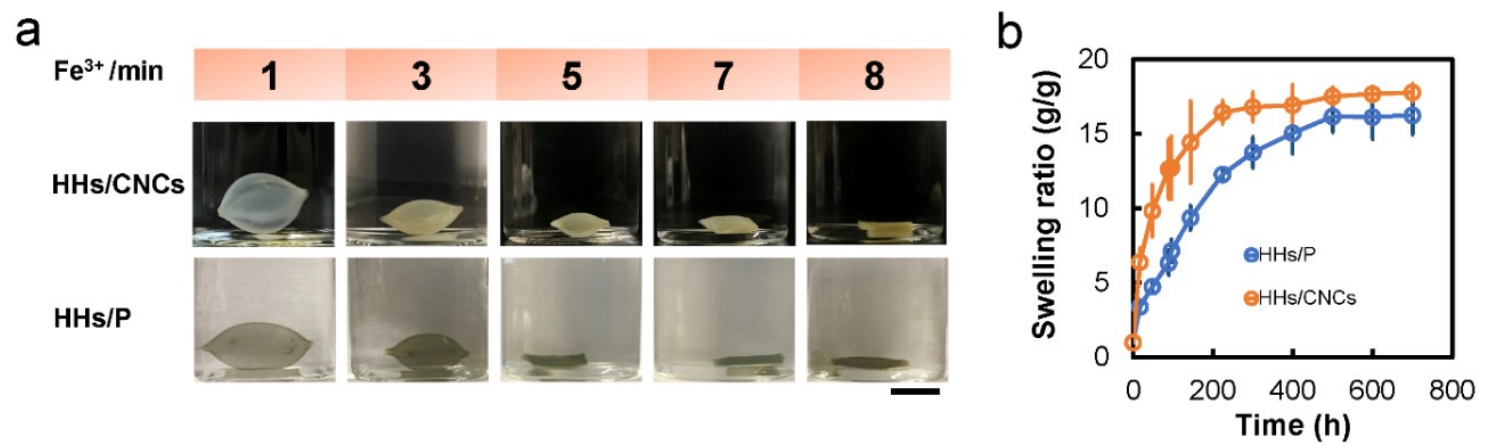

Figure 19. Formation process of hollow hydrogels with various $\mathrm{Fe}^{3+}$ immersing time. Scale bar: $1 \mathrm{~cm}$.

Besides the complex of $\mathrm{Fe}^{3+} /$ catechol, CNCs also play an important role for the formation of hollow hydrogels. CNCs with numerous carboxyl groups $(-\mathrm{COOH})$ on the surfaces significantly influenced the formation process of hollow hydrogels with respect to various immersion times in $\mathrm{Fe}^{3+}$ solution (Figure 19a). The dynamic hydrogels were immersed in $\mathrm{Fe}^{3+}$ solution for 1 to $8 \mathrm{~min}$ and then immediately transferred to DI water to dialyze. Obvious 
$\mathrm{HHs} / \mathrm{CNCs}$ were generated when treated by $\mathrm{Fe}^{3+}$ solution for 1 to $7 \mathrm{~min}$, while 8 min treatment would result to bulk solid core hydrogels due to the uniform strong crosslinking in the whole hydrogel. In comparison, without the addition of CNCs, hollow hydrogels were only appeared with 1 to 3 min treatment, while longer immersion time already resulted in bulk solid hydrogels. This evidence suggested the addition of CNCs could improve the spatial distinction within hydrogels, which can also be explained by the shorter formation time required to equilibrium during the evolution process. As shown in Figure 19b, HHs/CNCs with 3 minutes treatment of $\mathrm{Fe}^{3+}$ can reach the equilibrium after about 9 days, whereas, HHs/P needed roughly 20 days to equilibrium. Along with the diffusion rout of $\mathrm{Fe}^{3+}$ ions, $-\mathrm{COOH}$ on $\mathrm{CNCs}$ would complex with $\mathrm{Fe}^{3+}$ to provide additional crosslinking comparing with non-composite hydrogels. This extra crosslinking apparently retarded the penetration of $\mathrm{Fe}^{3+}$ ions, increasing the spatial crosslinking difference in hollow hydrogel formation.

Other than CNCs, different soaking times in $\mathrm{Fe}^{3+}$ solutions allowed steadily control of the wall thicknesses and their outer and inner surface morphologies as well as the microstructures in hollow hydrogels. Together with increasing the immersion time in $\mathrm{Fe}^{3+}$ solutions, the shell thickness of freeze-dried hollow hydrogels increased from roughly $0.22 \mathrm{~mm}$ to $0.75 \mathrm{~mm}$ (Figure 20a). At the same time, as displayed in SEM images, these freeze-dried hollow hydrogels after diverse immersion times in $\mathrm{Fe}^{3+}$ solutions had distinct outer and inner layers (Figure 20b). While the outer layers contained porous microstructures as typically for crosslinked networks in hydrogels, the inner layer contained a fluffy mat of loosely crosslinked polymer chains. This is because of different crosslinking densities in the outer and internal layers. Moreover, the pore sizes of outer surfaces in hollow hydrogels were largely decreased from $\sim 9.7 \pm 0.4 \mu \mathrm{m}$ to $\sim 3 \pm 0.5 \mu \mathrm{m}$ with increasing exposure time in $\mathrm{Fe}^{3+}$ solutions from 1 to 7 min, indicating further the formation of denser layers with longer crosslinking time. At the same time, the inner polymer mat became denser with longer treatment time in $\mathrm{Fe}^{3+}$ solutions and therefore increasing amount of penetrated $\mathrm{Fe}^{3+}$ ions. Furthermore, the outer and inner surface of the shells in dried $\mathrm{HHs} / \mathrm{CNCs}$ displayed different wettabilities, which partly depended on immersion times in $\mathrm{Fe}^{3+}$ solutions. The outer surfaces of HHs/CNCs became more hydrophobic because of increasing crosslinking densities with extended exposure time in $\mathrm{Fe}^{3+}$ 
solutions. In comparison, the inner surface was highly hydrophilic due to the fluffy polymer mats at room temperature (Figure 20b).

In accordance with more crosslinking after longer treatment in $\mathrm{Fe}^{3+}$ solutions, $\mathrm{HHs} / \mathrm{CNCs}$ with higher shell thicknesses became stronger (Figure 20c). HHs/CNCs with longer treatment in $\mathrm{Fe}^{3+}$ solutions ( 3 and 7 minutes) behaved similar as stiff hydrogels with larger deformations due to smaller internal volumes. In comparison, $\mathrm{HHs} / \mathrm{CNCs}$ with immersion in $\mathrm{Fe}^{3+}$ solution for 1 minute containing larger internal volume was softer and too brittle to bear compression.
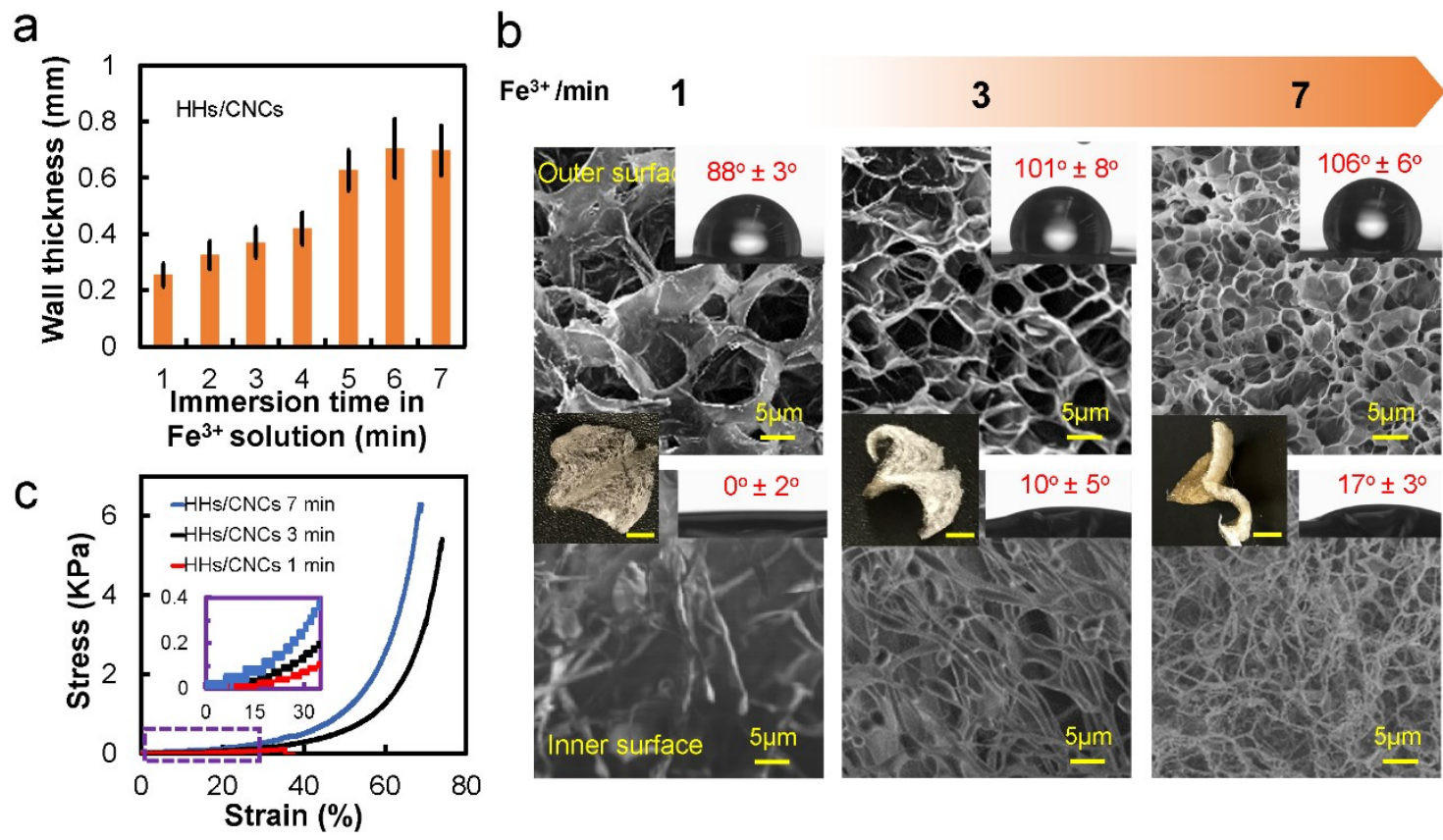

Figure 20. Regulation of microstructures and macrostructure of various $\mathrm{HHs} / \mathrm{CNCs}$. a) Shell thickness of $\mathrm{HHs} / \mathrm{CNCs}$ with increasing immersion times in aqueous $\mathrm{Fe}^{3+}$ solutions. b) SEM images and water contact angles showing microstructures and wetabilities of freeze-dried $\mathrm{HHs} / \mathrm{CNCs}$ with various treatment times in aqueous $\mathrm{Fe}^{3+}$ solutions. Insets are the photos of split freeze-dried HHs/CNCs with the scale bars of $2 \mathrm{~cm}$. c) Compression tests of HHs/CNCs with various treatment times in aqueous $\mathrm{Fe}^{3+}$ solutions. 


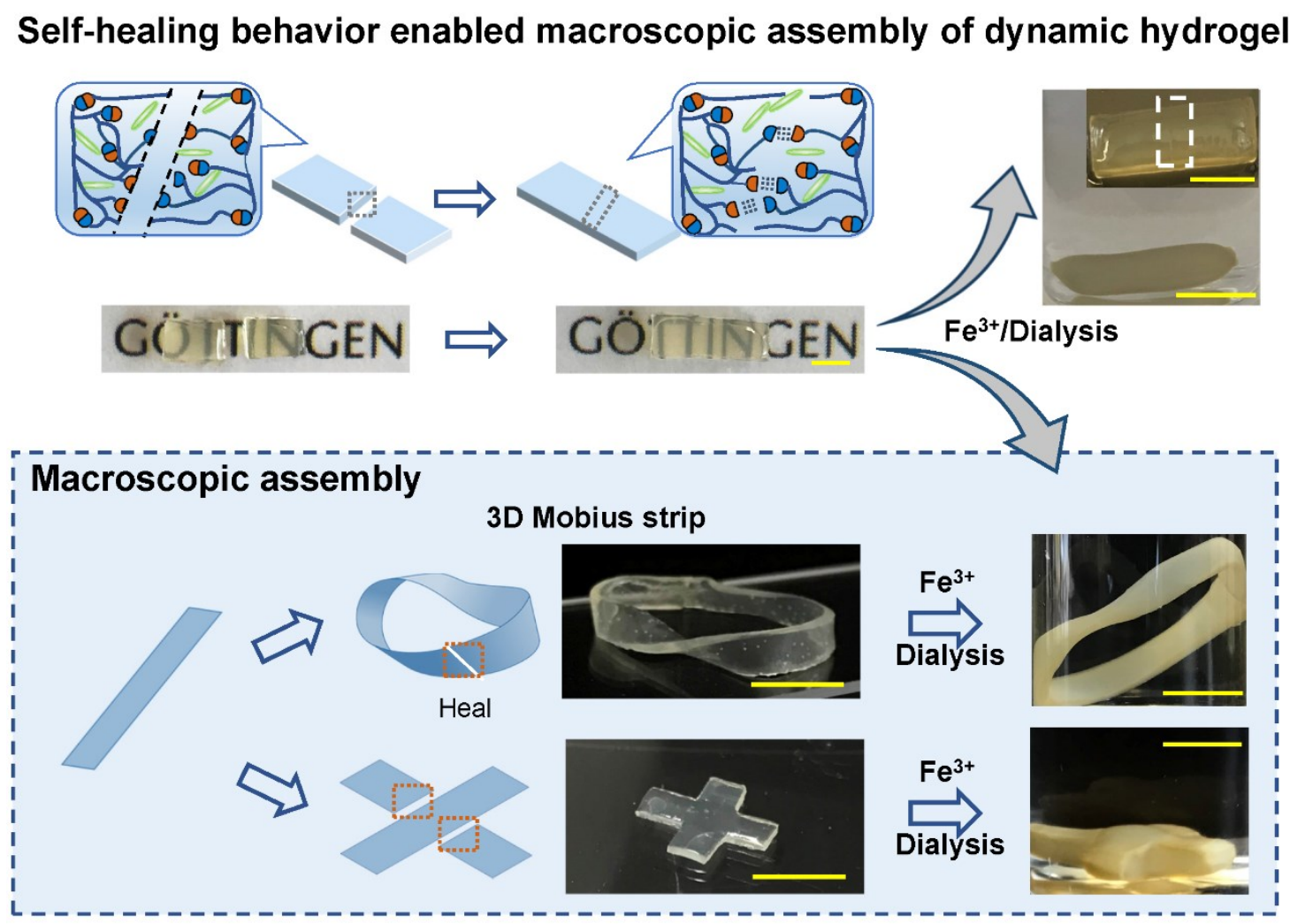

Figure 21. Shape programming of $\mathrm{HHs} / \mathrm{CNCs}$. Scale bar: $1 \mathrm{~cm}$.

More importantly, the CNCs composite hydrogels exhibited self-healing property owing to the dynamic boronate ester bonds (Figure 21). In particular, the self-healed dynamic hydrogels were still transformed into closed hollow hydrogels after subsequent treatment with $\mathrm{Fe}^{3+}$ ions and dialysis. Therefore, individual dynamic hydrogels can be regarded as building blocks for programming hollow hydrogels with more complex geometries, which can avoid the dependence on complicated molds for initial dynamic hydrogels and allow arbitrary shapeprogramming. For example, the flat hydrogel strip was twisted in 3D Mobius shape and the two ends were merged together by the self-healing properties of dynamic hydrogels. As well, three flat dynamic hydrogel strips merged together to give a cross-shaped dynamic hydrogel. They were further transformed into hollow hydrogels after $\mathrm{Fe}^{3+}$ treatment and dialysis. Therefore, this macroscopic assembly provides a new pathway for designing and programming multi-shaped hollow hydrogels. 

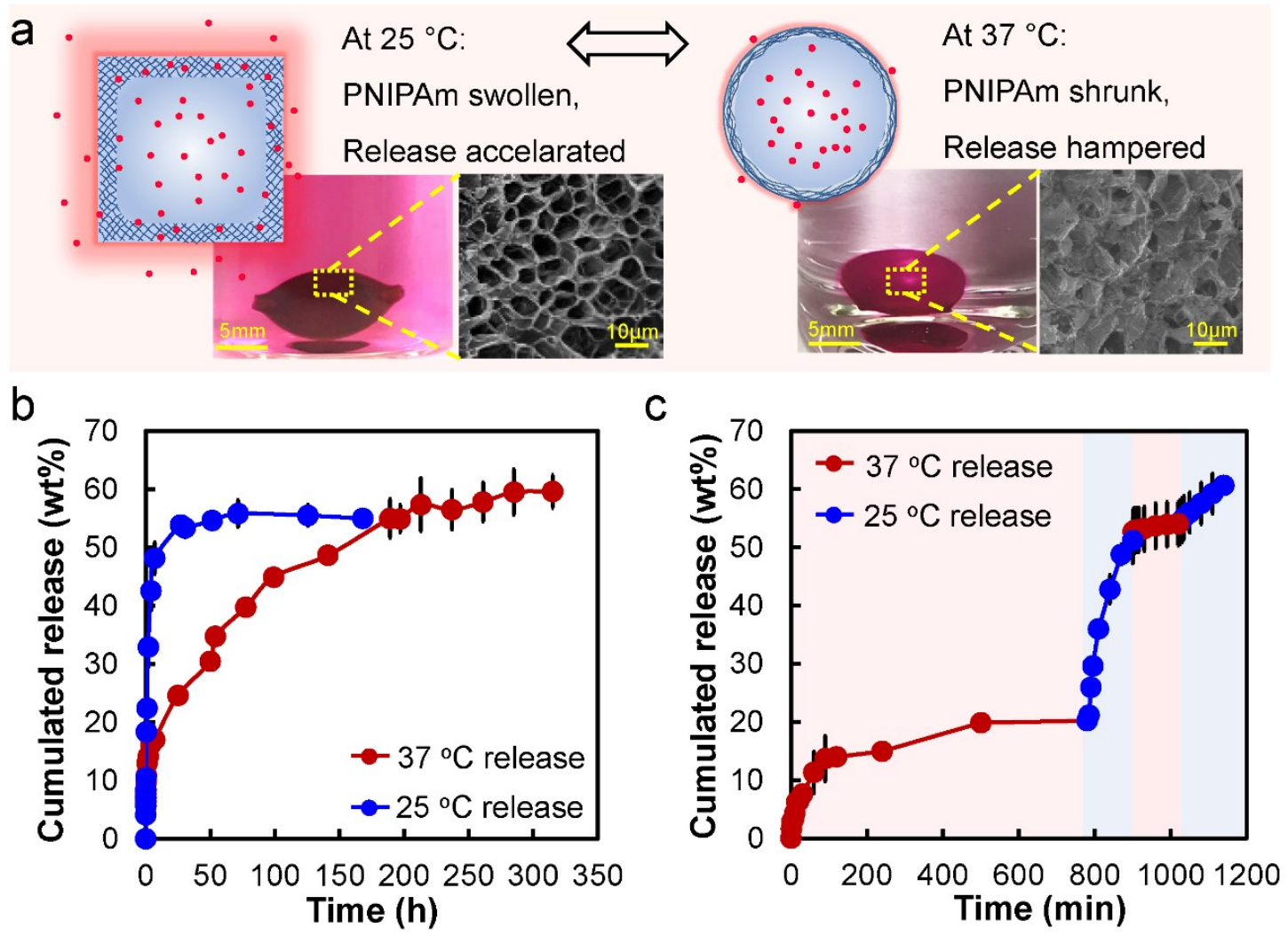

Figure 22. Temperature responsive release of water-soluble Rhodamine B.

Hollow hydrogels were then further used for the delivery of small molecular compounds due to their large interior volumes and LCST of PNIPAm. Different from common PNIPAm-based hydrogels as substrates, PNIPAm networks in hollow hydrogel walls represent tunable gates to provide different delivery behaviors. As demonstrated in Figure 22a, the HHs/CNCs loading with water-soluble Rhodamine B underwent a burst release at $25^{\circ} \mathrm{C}$ below LCST of PNIPAm with an open porous structure, in contrast, a sustained release at $37^{\circ} \mathrm{C}$ above LCST of PNIPAm, where the pores were "closed". Distinguished from high temperature burst release of bulk hydrogels with PNIPAm, the shrinkage of PNIPAm in hollow hydrogels was restricted by the large cavity of the aqueous core. Due to the presence of crosslinked PNIPAm primarily in the walls of hollow hydrogels, the increased temperature only resulted in shrinking pore size within the hydrogel walls, but little change in the overall volume. As a result, the release speed of Rhodamine B was significantly retarded by the compacted hydrogel walls.

Because the Rhodamine B solution in the cavity of hollow hydrogel was physically stuck by dense and hydrophobic wall at $37^{\circ} \mathrm{C}$. As proved in Figure 22b, the HHs/CNCs underwent a 
matrix release mode at $25{ }^{\circ} \mathrm{C}$, which was mainly driven by osmotic pressure. It would reach the release equilibrium after roughly $24 \mathrm{~h}$. On comparison, a sustained release mode was occurred at $37^{\circ} \mathrm{C}$ where the equilibrium was largely retarded to around $200 \mathrm{~h}$, further revealing the reduced pore size at $37{ }^{\circ} \mathrm{C}$. This is significantly different from typical PNIPAm-based materials as temperature-controlled release system with PNIPAm as substrate, where the large volume shrinkage could squeeze out the water as well as water-soluble agents above LCST. In addition, the release system can be well controlled by varying circumstance temperature (Figure 22c). At the beginning, the aqueous system was at $37^{\circ} \mathrm{C}$, a fast release was observed for the first 120 min arising from the release of Rhodamine B from shrinking wall. Afterward, only $6.2 \mathrm{wt} \%$ Rhodamine B could be released in the following $660 \mathrm{~min}(780-120)$ due to the largely reduced hydrogel pore size at $37{ }^{\circ} \mathrm{C}$. Once lowered the surrounding temperature to $25^{\circ} \mathrm{C}$, a burst release suddenly appeared with the opening pores in the wall of $\mathrm{HHs} / \mathrm{CNCs}$, in which $31 \mathrm{wt} \%$ of Rhodamine B was released in following $120 \mathrm{~min}$. These switchable release control between sustained/accelerated release functions repeatedly achieved.

In the present section, we demonstrated an in situ approach for macroscopic surface-interior separation from solid dynamic hydrogels to closed hollow hydrogels with tunable distinctive inner and outer surfaces of hydrogel shell. Based on the thermal responsive PNIPAm in shells, yet distinguished from conventional thermal controlled release systems, hollow hydrogels demonstrated a temperature-responsive gate system for sustained release at higher temperatures (above LCST) and accelerated release at lower temperatures.

\subsection{Structural Colors by Synergistic Birefringence and Surface Plasmon Resonance}

As reported in Publication 3, one-dimensional nanomaterials, the rod-like CNCs and gold nanorods (GNRs) were widely used in optical materials due to their respective inherent natures: birefringence with accompanying light retardation and surface plasmon resonance (SPR). We successfully anisotropically aligned them in polymer matrix based on the thixotropy of dynamic hydrogels, which synergistically leveraged the optical features of CNCs and GNRs 
into fabricating macroscopic optical films with broad color tunability.

a

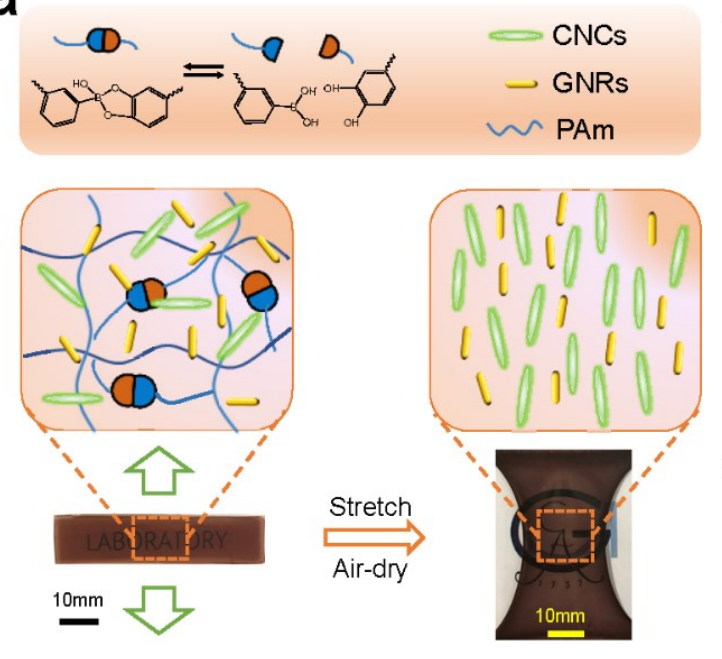

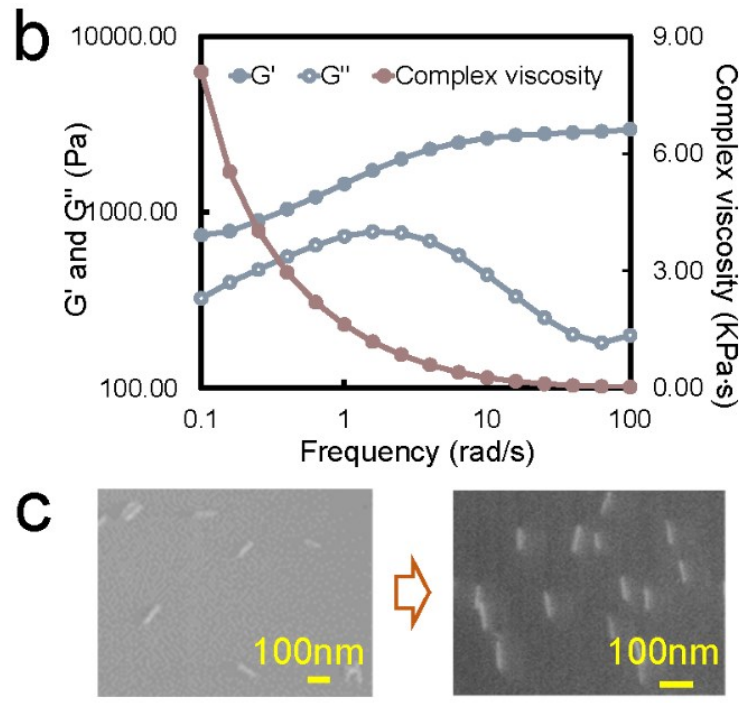

Figure 23. Preparation of CNCs/GNRs hybrid films with CNCs and GNRs aligned in a relaxed polymer matrix.

The CNCs/GNRs hybrid dynamic hydrogels were polymerized acrylamide crosslinked by phenylboronic acid/catechol complexes (PBA/DMA complexes) with pre-mixed CNCs and GNRs (Figure 23a). Because of the highly dynamic PBA/DMA complexes (associate constant $\mathrm{Ka}=0.919 \times 10^{3} \mathrm{M}^{-1}$ ), the mechanical properties of $\mathrm{CNCs} / \mathrm{GNRs}$ hybrid composite hydrogels were sensitive to the frequency of external loading (Figure 23b). The viscosity was decreased at higher frequency. Therefore, the rod-like nanoparticles can be aligned by this shear thinning behavior. During the uniaxial stretching, the crosslinking dissociated, leading to decreased complex viscosity, which allowed the anisotropic nanoparticles to unidirectional orient. Once stretching stopped and in the further air-drying process, PBA and DMA recombined to fix the orientation of CNCs and GNRs. As viewed from SEM images of air-dried films without and with stretching, the GNRs were aligned along stretching direction (Figure 23c). 

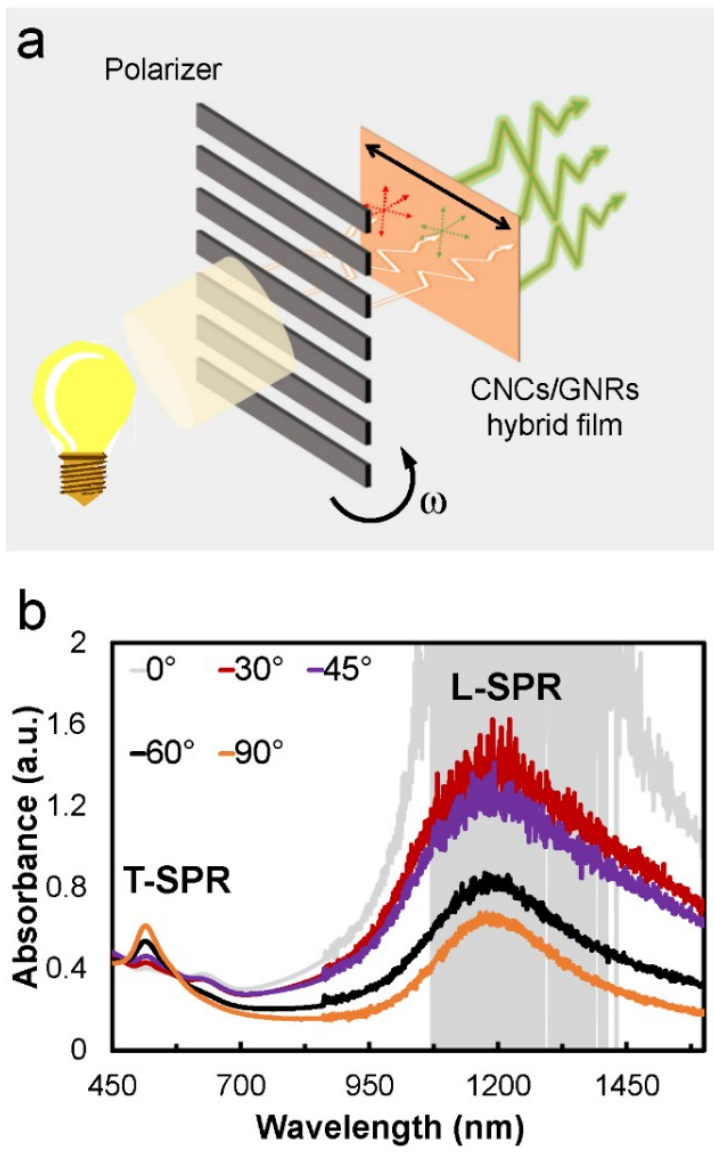

C
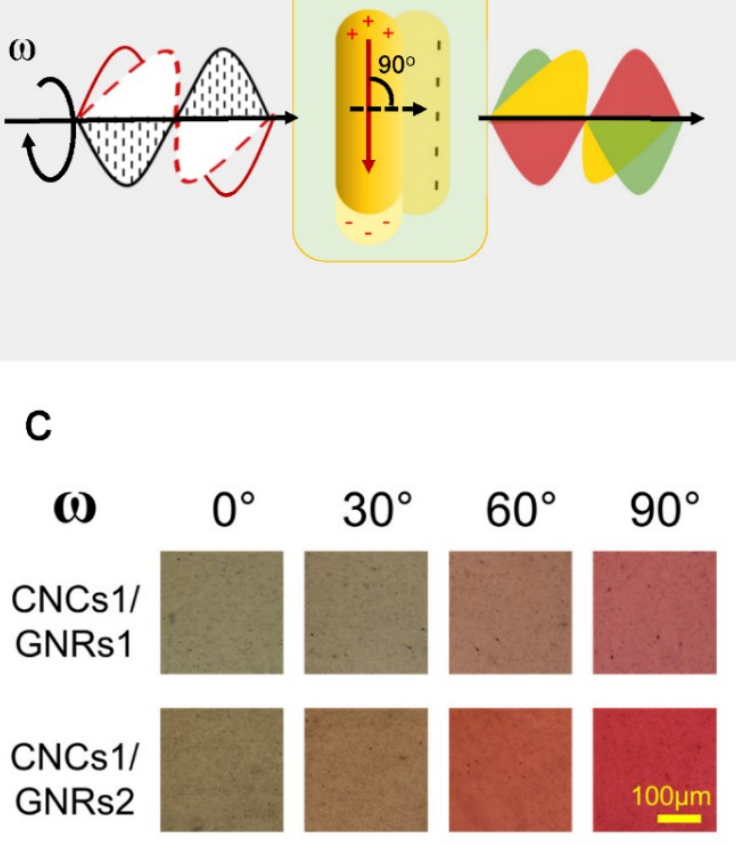

Figure 24. Schematic illustration for the regulation of SPR via varying light vibrating direction with rotating polarizer. Black arrow in the composite film represents stretching direction.

As illustrated in Figure 24a, the SPR of GNRs depended on the light vibrating direction with selective excitation of longitudinal surface plasmon resonance excitation(L-SPR) and transverse plasmon resonance (T-SPR) in a trading off rule. Varying the angles between the polarizer and the stretching direction of composite films (as defined as $\omega$ ), the angle between light vibrating direction to the longitudinal direction of GNRs can be changed. L-SPR is excited with light vibrating along the longitudinal direction of GNRs $\left(\omega=0^{\circ}\right)$, whereas light vibrating perpendicular to the longitudinal direction can excite T-SPR $\left(\omega=90^{\circ}\right)$. This phenomenon leads to light absorption at selective wavelengths with tunable intensity. Because of the well-ordered alignment of GNRs in the resulted polymer films, the SPR of single GNRs have been transferred to $\mathrm{CNCs} / \mathrm{GNRs}$ hybrid film in the macroscale without the influence of birefringence from CNCs when applied one polarizer. As shown in the ultraviolet-visible-near infrared (UVvis-NIR) spectra of CNCs1/GNRs1 hybrid film measuring with polarizer (Figure 24b), the 
absorption intensity at wavelength $520 \mathrm{~nm}$ (T-SPR) largely reduced, while that at wavelength $1150 \mathrm{~nm}$ (L-SPR) enhanced remarkably with rising $\omega$ from $0^{\circ}$ to $90^{\circ}$. For our naked eyes, the $520 \mathrm{~nm}$ light is corresponding to green colors. Therefore, its complementary color will be enhanced and show the structural color in red along with the absorption of light at $520 \mathrm{~nm}$ (Figure 24c). This phenomenon can be further reinforced by increasing the amount of GNRs in CNCs/GNRs hybrid films, which also improved the visible color brightness (Figure 24c).

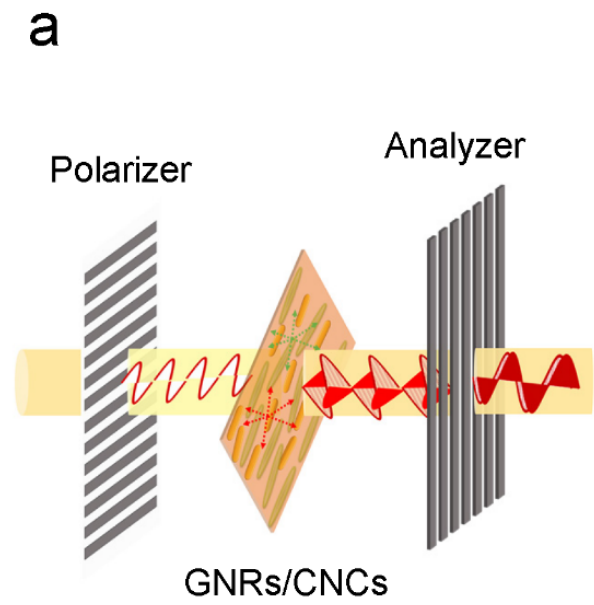

hybrid film
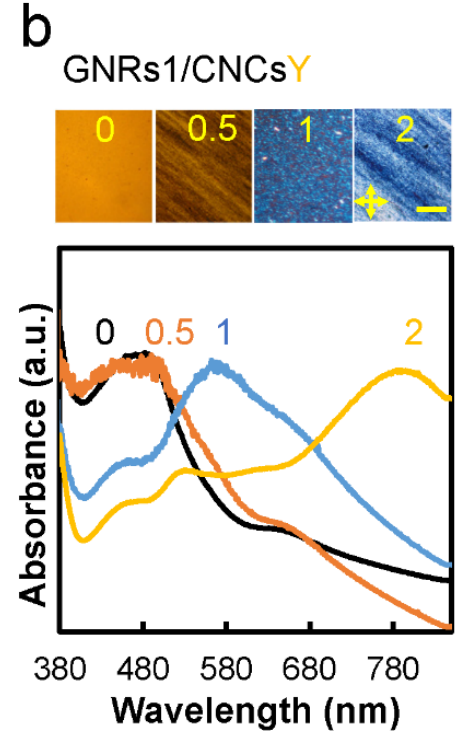

C CNCs1/GNRsX
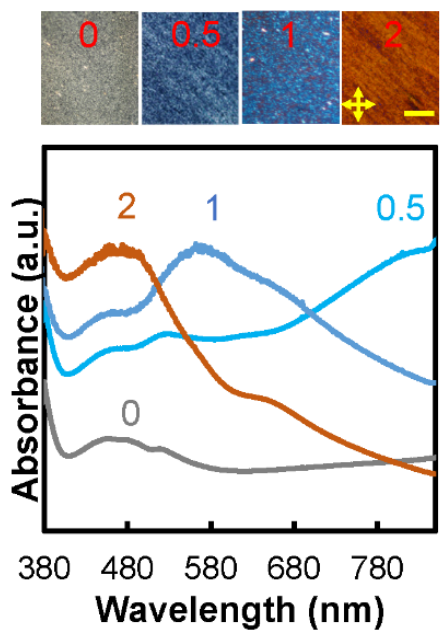

Figure 25. Integration of birefringence and SPR in CNCs/GNRs hybrid films. a) Schematic illustration of synergistic color adjustment between crossed polarizers. CNCs/GNRs hybrid films were placed at $45^{\circ}$ against crossed polarizer/analyzer. b-c) POM images and UV-vis spectra of CNCs/GNRs hybrid films measured between crossed polarizers with increasing contents of CNCs ( $\mathrm{Y}=0-2 \mathrm{wt} \%$ in initial hydrogel precursor solutions) and GNRs $(\mathrm{X}=0-2 \mathrm{wt} \%$ in initial hydrogel precursor solutions), respectively. Scale bar: $250 \mu \mathrm{m}$.

In addition to the effect of aligned GNRs on optical fields due to tunable light absorption intensities at around $520 \mathrm{~nm}$ and $1150 \mathrm{~nm}$ owing to modified SPR, the synergistic effect of ordered GNRs and CNCs on the structural colors of hybrid CNCs/GNRs films was further investigated. As schematically illustrated in Figure 25a, the interactions of the birefringence of CNCs and SPR of GNRs in hybrid films with incident light waves happen at the same time, when the polarized light irradiated on hybrid films. This synergistic effect generated bright vibrant colors when $\mathrm{CNCs} / \mathrm{GNRs}$ hybrid films were $45^{\circ}$ against crossed polarizer/analyzer. In 
order to classify their respective roles of SPR and birefringence in generating synergistic structural colors, we studied the structural colors of the hybrid films with fixed content of CNCs or GNRs in a fixed position of $45^{\circ}$ against crossed polarizer/analyzer, while changing the amount of the other.

With fixed GNRs content at $1 \mathrm{wt} \%$ in initial hydrogel precursor solutions, vibrant structural colors appeared varying from orange to blue with increasing CNCs concentration from 0 to 2 wt $\%$. The wavelength of absorbance peaks in the visible region of UV-vis spectra were red shifted from about $480 \mathrm{~nm}$ to $780 \mathrm{~nm}$ around (Figure 25b), which corresponded to observed structural colors from orange to blue. However, with fixed CNCs content and adding more GNRs, the wavelength of light absorption was blue shifted from about $860 \mathrm{~nm}$ to around 490 $\mathrm{nm}$ (Figure 25c). The visible colors were red shifted from light yellow to orange red with varying GNRs content from 0 to $2 \mathrm{wt} \%$. This was due to the varied light absorption for hybrid films with changing GNRs concentration at fixed CNCs concentration at $1 \mathrm{wt} \%$ in initial hydrogel precursor solutions. With increasing GNRs content, the T-SPR improved largely, while L-SPR only showed slight enhancement, indicating the GNRs mainly contributed to regulate the intensity of light absorption at wavelength of $520 \mathrm{~nm}$ within green light region (Figure 26).

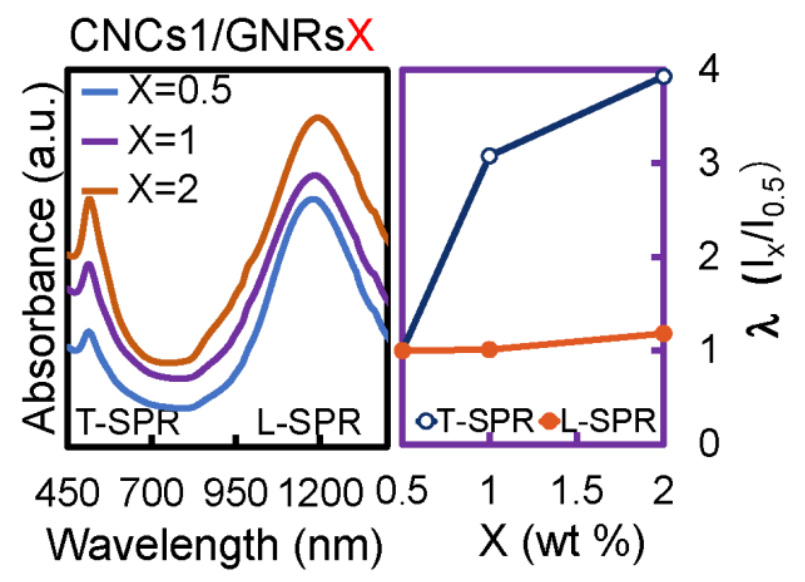

Figure 26. UV-vis-NIR spectra and the curve integrals of CNCs1/GNRsX hybrid films that were placed $45^{\circ}$ against one polarizer. Ix: Intensity of absorption peaks with various GNRs contents X. 


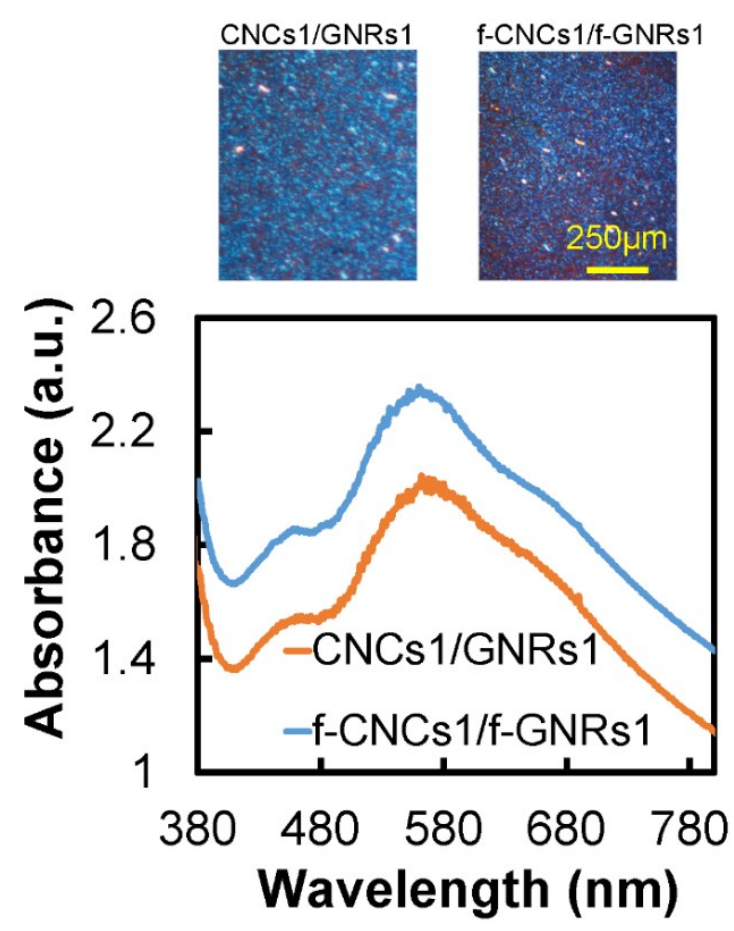

Figure 27. Structural colors and the UV-vis spectra of CNCs1/GNRs1 hybrid composite film and stacked f-CNCs1/f-GNRs1 films.

Because the structural colors between polarizers are not originated from polymer matrix due to the fast relaxation of polymer chains during stretching and drying, ${ }^{91}$ but rather from ordered CNCs and GNRs within the composite films, the physical location of these nanorods should not affect the resulting structural colors, as long as their organizations are equal. Therefore, structural colors of piled individual composite films with only CNCs (f-CNCs1) and only GNRs (f-GNRs1) separately in two films should be equivalent to those of hybrid films with both CNCs1/GNRs1 nanorods in the same films. In comparison, the separation of CNCs and GNRs in two separate films also provides the possibility to modularly construct the structure colors and to exactly elucidate their functions. By stacking f-GNRs and f-CNCs films along the stretching direction and placing them $45^{\circ}$ against the crossed polarizer/analyzer with $\mathrm{f}$ GNRs film facing the polarizer, diverse nearly equal colors as those of CNCs/GNRs hybrid films with the same contents of both nanorods were obtained. For instance, a CNCs1/GNRs 1 hybrid film had a similar absorption spectrum and exhibited highly similar loyal blue colors as those of piled films with the same contents of CNCs (f-CNCs1) and GNRs (f-GNRs1) (Figure 27). Piled f-CNCs1 and f-GNRs1 composite films were equivalent to CNCs1/GNRs1 hybrid 
films.

a
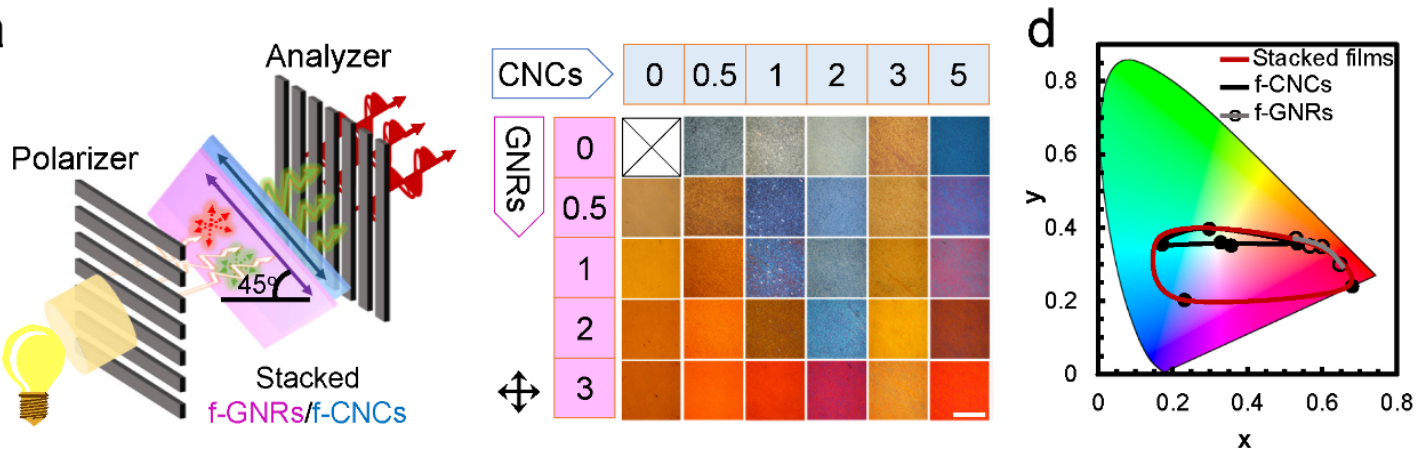

b Contribution of GNRs
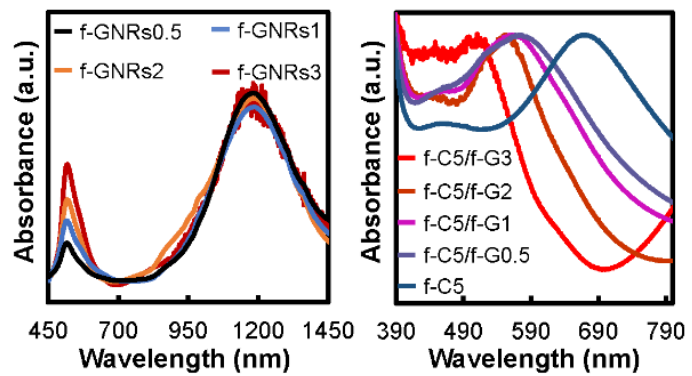

\section{Contribution of CNCs}
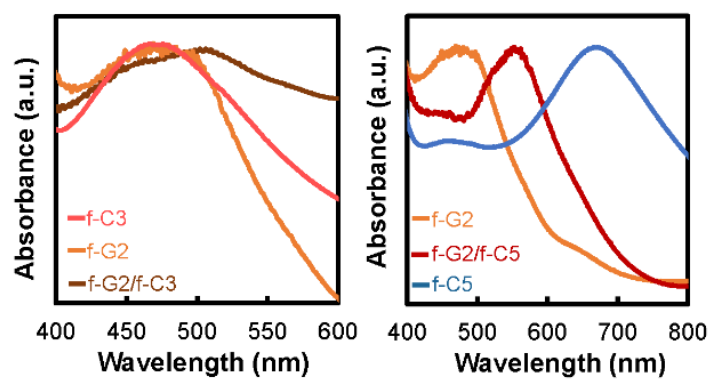

Figure 28. Color manipulation via stacking composite films f-GNRs and f-CNCs. f-C: abbreviation for f-CNCs, f-G: abbreviation for f-GNRs. Scale bar: $500 \mu \mathrm{m}$.

Moreover, these structural colors were gradually evolved to red colors of diverse intensities by increasing GNRs amount at constant CNCs concentrations (Figure 28a). This should be partially due to the enhanced light absorption at about $520 \mathrm{~nm}$, but negligible intensity changes at around $1150 \mathrm{~nm}$ (Figure 28b, left panel). Higher light absorption at about $520 \mathrm{~nm}$ improved the intensity of complementary red color in the resulting structural colors. In combination with the effect of birefringence by stacking f-CNCs5 film and f-GNRs films with increasing GNRs contents from 0 to $3 \mathrm{wt} \%$, wavelengths of light absorption peaks also exhibited a steady blueshift from about $665 \mathrm{~nm}$ to $510 \mathrm{~nm}$, corresponding to the change of more blue-green to red colors (Figure 28b, right panel).

Different from the SPR effect of GNRs, the structural colors in f-CNCs films were correlated to the accumulation of phase retardation originating from the concentration of aligned CNCs. Therefore, light retardation was further promoted by increasing CNCs amount and caused more interference colors. These $\mathrm{f}-\mathrm{CNCs}$ films with increasing initial CNCs concentrations from 0 to 
$5 \mathrm{wt} \%$ showed versatile structural colors appeared from grey over light yellow to blue-green colors (Figure 28a, right panel).

By stacking diverse f-CNCs films with the f-GNRs2 film, obtained structural colors were more diverse (Figure 28a, right panel). For instance, structural colors appeared from stacked fGNRs2/f-CNCs3 was orange with an overall light absorption peak at around $500 \mathrm{~nm}$, which was red-shifted compared to the light absorption peak of f-CNCs3 at $477 \mathrm{~nm}$ (Figure 28c). Piled f-GNRs2/f-CNCs5 films displayed red-violet color with the absorption peak at $550 \mathrm{~nm}$, which was blue-shifted from the absorption peak at $665 \mathrm{~nm}$ for f-CNCs5. These results demonstrated that the synergistic structural colors as well as wavelengths of absorption peaks for stacked f-GNRs and f-CNCs films strongly depended on the interference color and locations of absorption peaks of f-CNCs. Moreover, GNRs interacted with light by altering the intensity of light absorption at wavelength around $520 \mathrm{~nm}$ for the manipulation of synergistic structural colors, while the aligned CNCs produced periodic interference colors via light retardation.

Based on the integration of the two optical mechanisms, color tunability was apparently enlarged. As shown in Commission Internationale de l'éclairag (CIE) chart, the piled films covered a color range from blue to yellow-green and to violet, while f-CNCs and f-GNRs showed limited color regulation region (Figure 28d). Therefore, by separately adjusting the dosages of CNCs and GNRs in two piled individual films, the structural colors of stacked film piles can also be further manipulated similarly as the mixture of both in the same films (Figure 28a). In comparison, the physical separation of CNCs and GNRs in different films provided further flexibility for even broader color manipulation from $\mathrm{CNCs} / \mathrm{GNRs}$ dual model structure. 
a

Rotating f-GNRs of stacked f-GNRs/f-CNCs
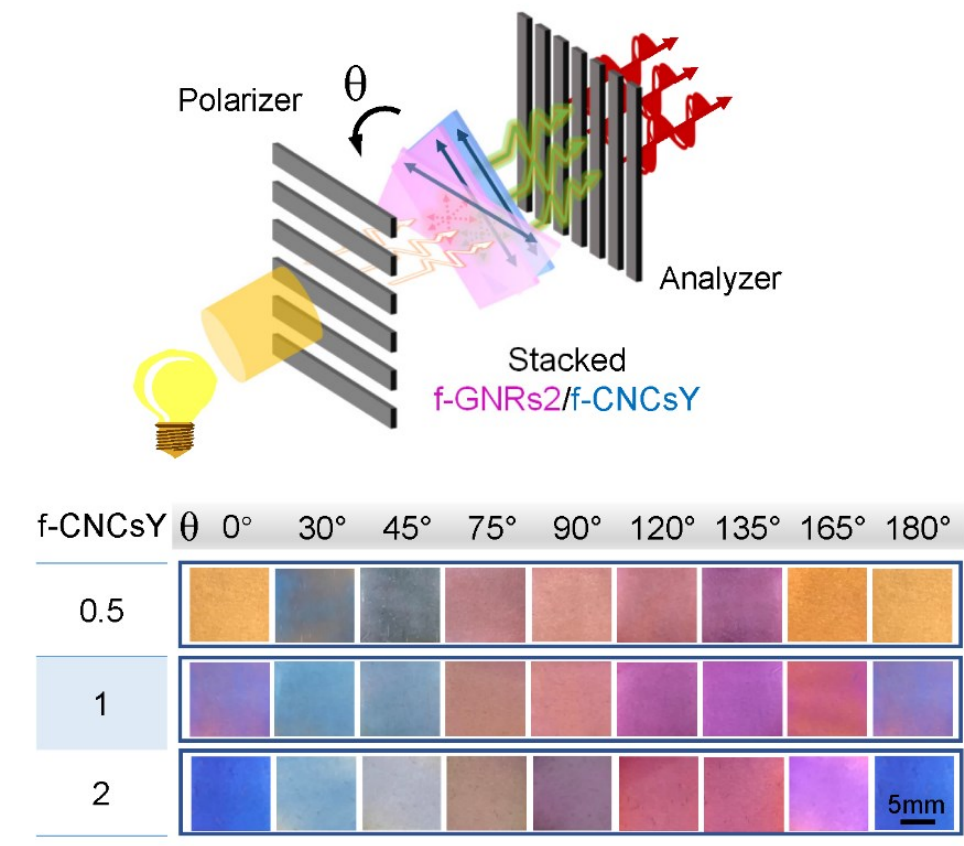

d Rotate f-GNRs2/f-CNCs1

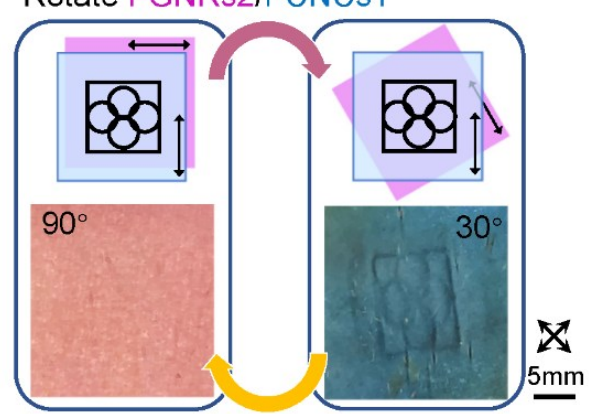

e

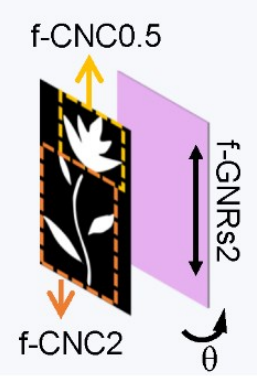

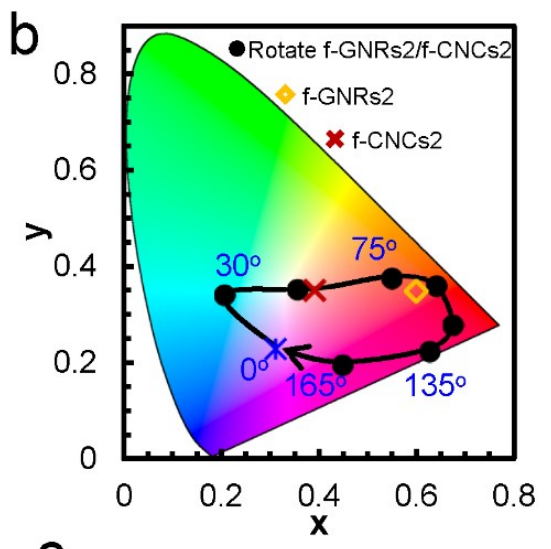

C
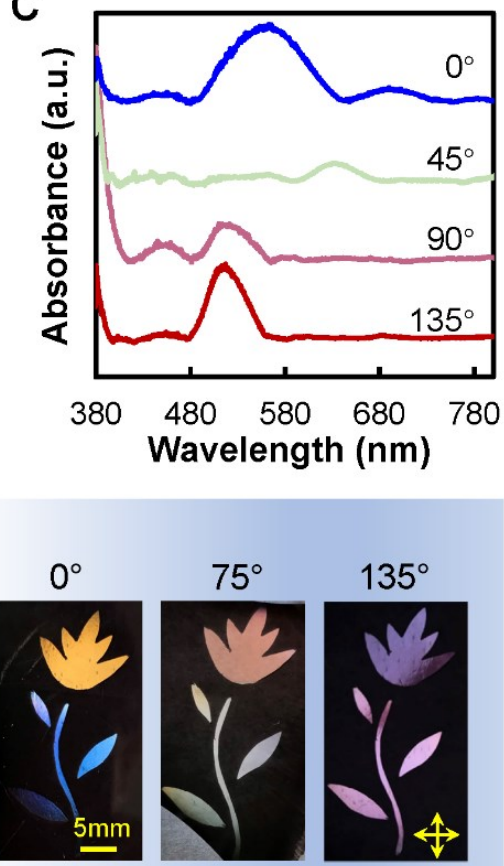

Figure 29. Tunable color manipulation via rotating f-GNRs films in stacked f-GNRs films and f-CNCs films.

In addition to adjusting the CNCs and GNRs contents in the individual films, rotating one film in the film piles demonstrated another strategy to generate a broad color range. In particular, this strategy can further provide more flexibility to tune the colors and save the preparation of diverse polymer films with various contents of CNCs or GNRs. As illustrated in Figure 29a, f-GNRs2 and various f-CNCs composite films were stacked along stretching direction and placed $45^{\circ}$ against the crossed polarizer/analyzer with f-GNRs2 film facing the polarizer. By rotating the f-GNRs2 film with a rotating angle $\theta$ between $\mathrm{f}-\mathrm{GNRs} 2$ film and $\mathrm{f}-\mathrm{CNCs}$ films of $0-180^{\circ}$, structural colors experienced successive color variation as blue, orange, red and purple 
(Figure 29a, bottom panel).

As shown in the CIE chart in Figure 29b, structural colors of the f-CNCs2/GNRs2 films after stacking and rotating covered a very broad color range from blue purple to cyan and gradually to yellow-green and red. As comparison, the structural colors of f-CNCs2, f-GNRs2 and their piled system without rotating only appeared as separate single dots in the CIE chart. Simply rotating the f-GNRs2 film of the stacked film piles strongly expanded the color types. Furthermore, the corresponding UV-vis spectra of stacked f-GNRs2 and f-CNCs2 with various rotating angles were measured to show the absorption changes during the rotating (Figure 29c). The dominating absorbance peaks can be readily controlled by rotating the f-GNRs 2 film, which was red-shifted from around $560 \mathrm{~nm}$ to $638 \mathrm{~nm}\left(0^{\circ}-45^{\circ}\right)$, then blue-shifted to $517 \mathrm{~nm}$ $\left(45^{\circ}-135^{\circ}\right)$, and eventually back to $560 \mathrm{~nm}\left(180^{\circ}\right)$. This evolution is corresponding to their sequential color change from blue-purple through cyan and red to blue-purple. This further demonstrated the flexible color regulation by adjusting the synergistic effect of SPR of GNRs and light retardation of CNCs. The light absorption at T-SPR and L-SPR of GNRs was altered by changing the incident light vibrating direction during the rotating (Figure 29a and Figure 24). Because of the presence of GNRs in the individual films, f-GNRs films functioned more like a further light filter before f-CNCs films, so that the wavelength of the incident lights after the f-GNRs film changed correspondingly. These incident lights with altered wavelengths further underwent light retardation through the f-CNCs films, leading to diverse colors. As a result, a much broader range of structural colors was achieved (Figure 29a).

Based on the broad color tunability and facile flexible construction of the stacking/rotating film piles, we developed a few prototypes of $\mathrm{f}-\mathrm{CNCs} / \mathrm{f}-\mathrm{GNRs}$ films for potential applications. As demonstrated in Figure 29d, a transparent f-CNCs1 composite film with a stamped pattern was prepared from the stamped hydrogel precursor and used with f-GNRs2 film as the stacked piles. By stacking both films perpendicular to each other $\left(90^{\circ}\right)$, in which the absorption of T-SPR of GNRs was minimized, the stamped pattern was not visible under POM. The stamped pattern appeared by rotating the f-GNRs 2 film to $30^{\circ}$ to the f-CNCs1 film, which indicated effective information encryption and readout on demand. Moreover, based on the abundant colors from 
diverse combinations of f-CNCs and f-GNRs films, optical films can be fabricated as tunable color display systems. As illustrated in Figure 29e, a variable color flower pattern was designed by stacking f-GNRs 2 film with f-CNCs0.5 film as the flower part and with f-CNCs2 film as the stalk and leaves. With the rotating f-GNRs2 film and altering light retardation due to various CNCs contents in f-CNCs films, the flower pattern exhibited distinct, adjustable color combinations. Based on these prototypes, these optical film systems with effective and flexible color control provide new platforms for the applications in information encryption and security systems, which have gained significant interest as novel security materials in the last years.

In the present work, we achieved the synergistic structural colors by combining the birefringence of CNCs and the SPR of GNRs within the composite films, which were embedded either in the same or in separate films. 


\section{General conclusion and perspectives}

As mentioned all along this study, the development of nanocomposite functional hydrogels based on natural polysaccharide nanocrystals has attracted growing interest. Among the polysaccharide nanocrystals, cellulose nanocrystals (CNCs) has attracted tremendous attention due to its inherent properties, including excellent mechanical properties, high aspect ratio, numerous surface-active groups and unique liquid crystal nature. Throughout this thesis, CNCs and surface modified CNCs were introduced to the synthetic hydrogels to fabricate functional hydrogels. Due to the modifiable surface and excellent mechanical strength, CNCs nanocrosslinkers can effectively improve the mechanical properties of composite hydrogels. In addition, the anisotropic shape and unique liquid crystal nature of CNCs provided the building block to construct optical polymer films with broad color tunability, basing on the thixotropy property of dynamic hydrogels.

Firstly, in Publication 1, we reported a series of bilayer hydrogel actuators (BHAs) comprising of various poly(N-hydroxyethyl acrylamide) (PHEAm) hydrogels as cooperating layers and poly(N-isopropylacrylamide) (PNIPAm) hydrogels as active layers. These BHAs demonstrated controllable thermal-responsive and solvent-driven actuation performance. The incorporation of neutral CNCs and methyl acrylamide modified CNCs (CNCs-MAm) in the cooperative PHEAm layers effectively improved the loading capacities of BHAs with stiffened PHEAm layers. The grabbed weight was promoted from $800 \mathrm{wt} \%$ to $1800 \mathrm{wt} \%$ of their own polymer weight, although the introduction of CNCs and CNCs-MAm reduced motion amplitude and response speed. The thermal-responsive property allowed BHAs to bend responding to the surrounding temperature, while the ethanol-driven actuation provides the feasibility to exactly tune the bending amplitude and bending direction of BHAs by adjusting the ethanol content. Therefore, these BHAs exhibited in fact the bidirectional actuation, which can be continuously altered by modifying the compositions of ethanol/water mixtures. Thus, these preprogrammable motions and the feasibility to spontaneously freeze the state of BHAs demonstrated their great peculiarity. Based on these advantageous properties, other stimuliresponsive materials with other functions can be simply used as the cooperating layer, which 
provides such BHAs vast potential for real-world applications at different scales.

Then, in Publication 2, we have demonstrated a in situ approach for transforming macroscopic solid dynamic hydrogel to closed hollow hydrogels with distinct inner and outer surface structures, via constructing a competitively crosslinking gradient within hydrogels. CNCs with numerous carboxyl groups $(-\mathrm{COOH})$ on the surface could increase the spatial distinction of the gradient crosslinking via additional weak crosslinking of $-\mathrm{COOH} / \mathrm{Fe}^{3+}$ complexes. This extra crosslinking apparently retarded the penetration of $\mathrm{Fe}^{3+}$ ions to the interior, which offered a time window to control the thickness and microstructures for the wall. The shapes of $3 \mathrm{D}$ hollow hydrogels can be programmed via the shapes' design of dynamic hydrogels. Furthermore, the dynamic crosslinking nature of hydrogels allowed macroscopic assembly via individual hydrogels to program the shape of closed hollow hydrogels. Based on the thermal responsive PNIPAm, yet distinguished from typical thermal controlled release systems, this hollow hydrogel showed a unique sustained release of hydrophilic small molecules at higher temperature. It would reach release equilibrium after only $\sim 24 \mathrm{~h}$ at $25^{\circ} \mathrm{C}$, on comparison, the release equilibrium was largely retarded to $\sim 200 \mathrm{~h}$ at $37^{\circ}$. This facile solid shaping methodology for fabricating hollow closed systems has great potential in diverse applications including controlled release, cell culture, chemical reactor and soft actuators.

Finally, in Publication 3, we successfully unidirectional aligned the one-dimensional nanomaterials, both CNCs and gold nanorods (GNRs) to the same hybrid composite films or separate films by means of dynamic precursor hydrogels. This demonstrated the integration of their inherent natures: birefringence of CNCs with accompanying light retardation and anisotropic surface plasmon resonance (SPR) of GNRs in manipulating structural colors. By synergistically leveraging the optical features of CNCs and GNRs with diverse amounts in hybrid films or in stacked individual films, wide-ranging structural colors were realized, far beyond the limitation of the same films solely with aligned CNCs or GNRs. Increasing GNRs contents led to promoted color red with enhanced light absorption at $520 \mathrm{~nm}$ and CNCs influenced the overall phase retardation, giving distinctively structural colors. Furthermore, with angle adjustment between CNCs films and GNRs films using stacking/rotating technique, 
we further achieve continuous color manipulation with more flexibility for color combinations. In one set of stacked films, light absorption wavelengths can traverse from roughly $500 \mathrm{~nm}$ to $650 \mathrm{~nm}$ solely by rotating f-GNRs film $\left(0-180^{\circ}\right)$. Tuning the adjustable synergism of the birefringence of CNCs and the SPR of GNRs in one film or separate films provides great potential for structural colors, which enlightens new avenue for optical applications.

In conclusion, the studies described in this thesis highlighted the possibility of using CNCs and surface modified CNCs as bio-based and versatile building blocks to functionalize synthetic hydrogels. For the mechanical reinforcement, the cross-linkable CNCs-MAm can significantly improve the stiffness of hydrogels, then effectively improved the loading capability for hydrogel actuators. Besides the mechanical reinforcement, the numerous carboxyl groups on the surface of neutral CNCs can associate with $\mathrm{Fe}^{3+}$ ions, which obviously improved the spatial difference of the strong cross-linking penetration gradient in hydrogel from outside to inside. This phenomenon assisted the transformation of solid to hollow hydrogel with tunable microstructures. Those hollow hydrogels fulfilled unique sustained release at higher temperature. In addition, by using the liquid behaviors of dynamic hydrogels, one-dimensional nanomaterials, CNCs and GNRs can be aligned into relaxed polymer films to fabricate optical films with broad color tunability. Thus, dynamic composite hydrogels can be used as precursors to program dry polymers with various structural colors. Specially, the unique optical properties of both CNCs and GNRs can be solely exhibited and integrated to manipulate structural colors.

According to the studies in this thesis, the CNCs composite hydrogels not only reinforced the synthetic hydrogels with improved loading performance in certain usage, but also introduced novel functions to composite hydrogel by building new secondary microstructures within hydrogels. In the future, more efforts need to be done to construct strong hydrogel actuators with fast response speed and large amplitude based on CNCs. Furthermore, thanks to the modifiable surface of CNCs, diverse functional groups or polymers can be introduced to them, which provides the possibility to involve various functions to hydrogels. 


\section{References}

1. Zhang, Y. S.; Khademhosseini, A., Advances in engineering hydrogels. Science 2017, 356, 3627.

2. Sun, T. L.; Kurokawa, T.; Kuroda, S.; Ihsan, A. B.; Akasaki, T.; Sato, K.; Haque, M. A.; Nakajima, T.; Gong, J. P., Physical hydrogels composed of polyampholytes demonstrate high toughness and viscoelasticity. Nature Materials 2013, 12, 932.

3. Kim, S.; Peterson, A. M.; Holten-Andersen, N., Enhanced water retention maintains energy dissipation in dehydrated metal-coordinate polymer networks: another role for Fecatechol cross-links? Chemistry of Materials 2018, 30, 3648.

4. Hines, L.; Petersen, K.; Lum, G. Z.; Sitti, M., Soft actuators for small-scale robotics. Advanced Materials 2017, 29, 1603483.

5. Feng, Q.; Lin, S.; Zhang, K.; Dong, C.; Wu, T.; Huang, H.; Yan, X.; Zhang, L.; Li, G.; Bian, L., Sulfated hyaluronic acid hydrogels with retarded degradation and enhanced growth factor retention promote hMSC chondrogenesis and articular cartilage integrity with reduced hypertrophy. Acta Biomaterialia 2017, 53, 329.

6. Liu, X.; Wu, D.; Wang, H.; Wang, Q., Self-recovering tough gel electrolyte with adjustable supercapacitor performance. Advanced Materials 2014, 26, 4370.

7. Wang, L.; Zhong, C.; Ke, D.; Ye, F.; Tu, J.; Wang, L.; Lu, Y., Ultrasoft and highly stretchable hydrogel optical fibers for in vivo optogenetic modulations. Advanced Optical Materials 2018, 6, 1800427.

8. Chen, T.; Chen, Y.; Rehman, H. U.; Chen, Z.; Yang, Z.; Wang, M.; Li, H.; Liu, H., Ultratough, self-healing, and tissue-adhesive hydrogel for wound dressing. ACS Applied Materials \& Interfaces 2018, 10, 33523.

9. Feng, J.; Ton, X. A.; Zhao, S.; Paez, J.; del Campo, A., Mechanically reinforced catecholcontaining hydrogels with improved tissue gluing performance. Biomimetics 2017, 2, 23.

10. Caliari, S. R.; Burdick, J. A., A practical guide to hydrogels for cell culture. Nature Methods 2016, 13, 405.

11. Jeon, O.; Wolfson, D. W.; Alsberg, E., In-situ formation of growth-factor-loaded coacervate microparticle-embedded hydrogels for directing encapsulated stem cell fate. 
Advanced Materials 2015, 27, 2216.

12. Lei, Z.; Wang, Q.; Wu, P., A multifunctional skin-like sensor based on a 3D printed thermoresponsive hydrogel. Materials Horizons 2017, 4, 694.

13. Lin, S.; Yuk, H.; Zhang, T.; Parada, G. A.; Koo, H.; Yu, C.; Zhao, X., Stretchable hydrogel electronics and devices. Advanced Materials 2016, 28, 4497.

14. Kim, Y. S.; Liu, M.; Ishida, Y.; Ebina, Y.; Osada, M.; Sasaki, T.; Hikima, T.; Takata, M.; Aida, T., Thermoresponsive actuation enabled by permittivity switching in an electrostatically anisotropic hydrogel. Nature Materials 2015, 14, 1002.

15. Ionov, L., Hydrogel-based actuators: possibilities and limitations. Materials Today 2014, $17,494$.

16. Lee, B. P.; Konst, S., Novel hydrogel actuator inspired by reversible mussel adhesive protein chemistry. Advanced Materials 2014, 26, 3415.

17. Kamata, H.; Akagi, Y.; Kayasuga-Kariya, Y.; Chung, U.-i.; Sakai, T., "Nonswellable" hydrogel without mechanical hysteresis. Science 2014, 343, 873.

18. Kamata, H.; Kushiro, K.; Takai, M.; Chung, U.-i.; Sakai, T., Non-osmotic hydrogels: a rational strategy for safely degradable hydrogels. Angewandte Chemie International Edition 2016, 55, 9282.

19. Shastri, A.; McGregor, L. M.; Liu, Y.; Harris, V.; Nan, H.; Mujica, M.; Vasquez, Y.; Bhattacharya, A.; Ma, Y.; Aizenberg, M.; Kuksenok, O.; Balazs, A. C.; Aizenberg, J.; He, X., An aptamer-functionalized chemomechanically modulated biomolecule catch-and-release system. Nature Chemistry 2015, 7, 447.

20. Hill, R. J. Hydrogel charge regulation and electrolyte ion concentration perturbations in nanoparticle gel electrophoresis. Proceedings of the Royal Society A: Mathematical, Physical and Engineering Sciences, 2015, 471, 20150523.

21. Usuki, K.; Onoe, H.; Iwase, E., Deformable coloring element using an electroactive hydrogel with bottom-arranged electrodes. Japanese Journal of Applied Physics 2015, 54, 06FP06.

22. Gao, X.; Cao, Y.; Song, X.; Zhang, Z.; Xiao, C.; He, C.; Chen, X., pH- and thermoresponsive poly( $\mathrm{N}$-isopropylacrylamide-co-acrylic acid derivative) copolymers and hydrogels 
with LCST dependent on pH and alkyl side groups. Journal of Materials Chemistry B 2013, 1, 5578 .

23. Zhao, F.; Zhou, X.; Liu, Y.; Shi, Y.; Dai, Y.; Yu, G., Super moisture-absorbent gels for allweather atmospheric water harvesting. Advanced Materials 2019, 31, 1806446.

24. Zheng, L.; Shi, L.; Sun, P.; Ren, S.; Liu, F.; Wu, A.; Sun, N., Smart low molecular weight hydrogels with dynamic covalent skeleton. Soft Matter 2018, 14, 6678.

25. Lakes, A. L.; Jordan, C. T.; Gupta, P.; Puleo, D. A.; Hilt, J. Z.; Dziubla, T. D., Reducible disulfide poly(beta-amino ester) hydrogels for antioxidant delivery. Acta Biomaterialia 2018, $68,178$.

26. Harada, A.; Takashima, Y.; Nakahata, M., Supramolecular polymeric materials via cyclodextrin-guest interactions. Accounts of Chemical Research 2014, 47, 2128.

27. Lee, I.-N.; Dobre, O.; Richards, D.; Ballestrem, C.; Curran, J. M.; Hunt, J. A.; Richardson, S. M.; Swift, J.; Wong, L. S., Photoresponsive hydrogels with photoswitchable mechanical properties allow time-resolved analysis of cellular responses to matrix stiffening. ACS Applied Materials \& Interfaces 2018, 10, 7765.

28. Vales, T. P.; Badon, I. W. T.; Kim, H.-J., Multi-responsive hydrogels functionalized with a photochromic spiropyran-conjugated chitosan network. Macromolecular Research 2018, 26, 950.

29. Francis, W.; Dunne, A.; Delaney, C.; Florea, L.; Diamond, D., Spiropyran based hydrogels actuators - walking in the light. Sensors and Actuators B: Chemical 2017, 250, 608.

30. Cromwell, O. R.; Chung, J.; Guan, Z., Malleable and self-healing covalent polymer networks through tunable dynamic boronic ester bonds. Journal of the American Chemical Society 2015, 137, 6492.

31. Rao, P.; Sun, T. L.; Chen, L.; Takahashi, R.; Shinohara, G.; Guo, H.; King, D. R.; Kurokawa, T.; Gong, J. P., Tough hydrogels with fast, strong, and reversible underwater adhesion based on a multiscale design. Advanced Materials 2018, 30, 1801884.

32. Iwaso, K.; Takashima, Y.; Harada, A., Fast response dry-type artificial molecular muscles with [c2] daisy chains. Nature Chemistry 2016, 8, 625.

33. Hou, S.; Ma, P. X., Stimuli-responsive supramolecular hydrogels with high extensibility 
and fast self-healing via precoordinated mussel-inspired chemistry. Chemistry of Materials 2015, $27,7627$.

34. Le, X.; Lu, W.; Xiao, H.; Wang, L.; Ma, C.; Zhang, J.; Huang, Y.; Chen, T., Fe ${ }^{3+}-$, pH-, thermoresponsive supramolecular hydrogel with multishape memory effect. ACS Applied Materials \& Interfaces 2017, 9, 9038.

35. Tian, K.; Bae, J.; Bakarich, S. E.; Yang, C.; Gately, R. D.; Spinks, G. M.; In Het Panhuis, M.; Suo, Z.; Vlassak, J. J., 3D printing of transparent and conductive heterogeneous hydrogelelastomer systems. Advanced Materials 2017, 29, 1604827.

36. Duan, J.; Xie, W.; Yang, P.; Li, J.; Xue, G.; Chen, Q.; Yu, B.; Liu, R.; Zhou, J., Tough hydrogel diodes with tunable interfacial adhesion for safe and durable wearable batteries. Nano Energy 2018, 48, 569.

37. Schroeder, T. B. H.; Guha, A.; Lamoureux, A.; VanRenterghem, G.; Sept, D.; Shtein, M.; Yang, J.; Mayer, M., An electric-eel-inspired soft power source from stacked hydrogels. Nature 2017, 552, 214.

38. Zhu, M.; Wang, X.; Tang, H.; Wang, J.; Hao, Q.; Liu, L.; Li, Y.; Zhang, K.; Schmidt, O. G., Antifreezing hydrogel with high zinc reversibility for flexible and durable aqueous batteries by cooperative hydrated cations. Advanced Functional Materials 2020, 30, 1907218.

39. Horning, M.; Nakahata, M.; Linke, P.; Yamamoto, A.; Veschgini, M.; Kaufmann, S.; Takashima, Y.; Harada, A.; Tanaka, M., Dynamic Mechano-regulation of myoblast cells on supramolecular hydrogels cross-linked by reversible host-guest interactions. Scientific Reports 2017, 7, 7660 .

40. Bai, H.; Polini, A.; Delattre, B.; Tomsia, A. P., Thermoresponsive composite hydrogels with aligned macroporous structure by ice-templated assembly. Chemistry of Materials 2013, 25,4551 .

41. Osorio-Madrazo, A.; Eder, M.; Rueggeberg, M.; Pandey, J. K.; Harrington, M. J.; Nishiyama, Y.; Putaux, J. L.; Rochas, C.; Burgert, I., Reorientation of cellulose nanowhiskers in agarose hydrogels under tensile loading. Biomacromolecules 2012, 13, 850.

42. Wang, Z. J.; Zhu, C. N.; Hong, W.; Wu, Z. L.; Zheng, Q., Cooperative deformations of periodically patterned hydrogels. Science Advances 2017, 3, 1700348. 
43. Jiang, S.; Liu, F.; Lerch, A.; Ionov, L.; Agarwal, S., Unusual and superfast temperaturetriggered actuators. Advanced Materials 2015, 27, 4865 .

44. Yao, C.; Liu, Z.; Yang, C.; Wang, W.; Ju, X. J.; Xie, R.; Chu, L. Y., Smart hydrogels with inhomogeneous structures assembled using nanoclay-cross-linked hydrogel subunits as building blocks. ACS Applied Materials \& Interfaces 2016, 8, 21721.

45. Duan, J.; Liang, X.; Zhu, K.; Guo, J.; Zhang, L., Bilayer hydrogel actuators with tight interfacial adhesion fully constructed from natural polysaccharides. Soft Matter 2017, 13, 345. 46. Wang, Z. J.; Hong, W.; Wu, Z. L.; Zheng, Q., Site-specific pre-swelling-directed morphing structures of patterned hydrogels. Angewandte Chemie International Edition 2017, 56, 15974. 47. Palleau, E.; Morales, D.; Dickey, M. D.; Velev, O. D., Reversible patterning and actuation of hydrogels by electrically assisted ionoprinting. Nature Communications 2013, 4, 2257.

48. Han, L.; Zheng, Y.; Luo, H.; Feng, J.; Engstler, R.; Xue, L.; Jing, G.; Deng, X.; Del Campo, A.; Cui, J., Macroscopic self-evolution of dynamic hydrogels to create hollow interiors. Angewandte Chemie 2019, 132, 5660.

49. Ouyang, L.; Burdick, J. A.; Sun, W., Facile biofabrication of heterogeneous multilayer tubular hydrogels by fast diffusion-induced gelation. ACS Applied Materials \& Interfaces 2018, $10,12424$.

50. Mredha, M. T. I.; Guo, Y. Z.; Nonoyama, T.; Nakajima, T.; Kurokawa, T.; Gong, J. P., A Facile method to fabricate anisotropic hydrogels with perfectly aligned hierarchical fibrous structures. Advanced Materials 2018, 30, 1704937.

51. Liu, L.; Jiang, S.; Sun, Y.; Agarwal, S., Giving direction to motion and surface with ultrafast speed using oriented hydrogel fibers. Advanced Functional Materials 2016, 26, 1021.

52. Buabeid, M. A.; Arafa, E.-S. A.; Murtaza, G., Emerging prospects for nanoparticle-enabled cancer immunotherapy. Journal of Immunology Research 2020, 2020, 1.

53. Esmaeely Neisiany, R.; Enayati, M. S.; Sajkiewicz, P.; Pahlevanneshan, Z.; Ramakrishna, S., Insight into the current directions in functionalized nanocomposite hydrogels. Frontiers in Materials 2020, 7, 25.

54. Mehrali, M.; Thakur, A.; Pennisi, C. P.; Talebian, S.; Arpanaei, A.; Nikkhah, M.; Dolatshahi-Pirouz, A., Nanoreinforced hydrogels for tissue engineering: biomaterials that are 
compatible with load-bearing and electroactive tissues. Advanced Materials 2017, 29, 1603612. 55. Šupová, M.; Martynková, G. S.; Barabaszová, K., Effect of nanofillers dispersion in polymer matrices: a review. Science of advanced materials 2011, 3, 1.

56. Wang, Q.; Gao, Z., A constitutive model of nanocomposite hydrogels with nanoparticle crosslinkers. Journal of the Mechanics and Physics of Solids 2016, 94, 127.

57. Luo, F.; Sun, T. L.; Nakajima, T.; Kurokawa, T.; Zhao, Y.; Sato, K.; Ihsan, A. B.; Li, X.; Guo, H.; Gong, J. P., Oppositely charged polyelectrolytes form tough, self-healing, and rebuildable hydrogels. Advanced Materials 2015, 27, 2722.

58. Appel, E. A.; Biedermann, F.; Hoogland, D.; Del Barrio, J.; Driscoll, M. D.; Hay, S.; Wales, D. J.; Scherman, O. A., Decoupled associative and dissociative processes in strong yet highly dynamic host-guest complexes. Journal of the American Chemical Society 2017, 139, 12985. 59. Mozhdehi, D.; Ayala, S.; Cromwell, O. R.; Guan, Z., Self-healing multiphase polymers via dynamic metal-ligand interactions. Journal of the American Chemical Society 2014, 136, 16128.

60. Zhao, F.; Yao, D.; Guo, R.; Deng, L.; Dong, A.; Zhang, J., Composites of polymer hydrogels and nanoparticulate systems for biomedical and pharmaceutical applications. Nanomaterials 2015, 5, 2054.

61. Lampe, K. J.; Kern, D. S.; Mahoney, M. J.; Bjugstad, K. B., The administration of BDNF and GDNF to the brain via PLGA microparticles patterned within a degradable PEG-based hydrogel: Protein distribution and the glial response. Journal of Biomedical Materials Research Part A 2011, 96, 595.

62. Rafieian, S.; Mirzadeh, H.; Mahdavi, H.; Masoumi Mir, E., A review on nanocomposite hydrogels and their biomedical applications. Science and Engineering of Composite Materials 2019, 26, 154.

63. Mittal, V., Carbon nanotubes surface modifications: an overview. Surface Modification of Nanotube Fillers 2011, 1.

64. Eitan, A.; Jiang, K.; Dukes, D.; Andrews, R.; Schadler, L. S., Surface modification of multiwalled carbon nanotubes: toward the tailoring of the interface in polymer composites. Chemistry of Materials 2003, 15, 3198. 
65. Wang, X.; Zhang, C.; Du, Z.; Li, H.; Zou, W., Synthesis of non-destructive amido group functionalized multi-walled carbon nanotubes and their application in antistatic and thermal conductive polyetherimide matrix nanocomposites. Polymers for Advanced Technologies 2017, 28,791 .

66. Sun, X.; Qin, Z.; Ye, L.; Zhang, H.; Yu, Q.; Wu, X.; Li, J.; Yao, F., Carbon nanotubes reinforced hydrogel as flexible strain sensor with high stretchability and mechanically toughness. Chemical Engineering Journal 2020, 382, 122832.

67. Yu, X.; Li, Y.; Yang, J.; Chen, F.; Tang, Z.; Zhu, L.; Qin, G.; Dai, Y.; Chen, Q., Nanoclay reinforced self-cross-linking poly(N-hydroxyethyl acrylamide) hydrogels with integrated high performances. Macromolecular Materials and Engineering 2018, 303, 1800295.

68. Li, H. J.; Jiang, H.; Haraguchi, K., Ultrastiff, thermoresponsive nanocomposite hydrogels composed of ternary polymer-clay-silica networks. Macromolecules 2018, 51, 529.

69. Tan, H. L.; Teow, S. Y.; Pushpamalar, J., Application of metal nanoparticle hydrogel composites in tissue regeneration. Bioengineering 2019, 6, 17.

70. Sun, Z.; Yamauchi, Y.; Araoka, F.; Kim, Y. S.; Bergueiro, J.; Ishida, Y.; Ebina, Y.; Sasaki, T.; Hikima, T.; Aida, T., An anisotropic hydrogel actuator enabling earthworm-like directed peristaltic crawling. Angewandte Chemie International Edition 2018, 57, 15772.

71. Fajardo, A. R.; Pereira, A. G.; Muniz, E. C., Hydrogels nanocomposites based on crystals, whiskers and fibrils derived from biopolymers. Eco-friendly Polymer Nanocomposites. Springer, 2015, 43.

72. Huang, J., Chang, P. R., Lin, N., Dufresne, A., Polysaccharide-based nanocrystals: chemistry and applications. John Wiley \& Sons, 2014.

73. López-López, E. A.; Hernández-Gallegos, M. A.; Cornejo-Mazón, M.; HernándezSánchez, H., Polysaccharide-based nanoparticles. Food Nanoscience and Nanotechnology. Springer, Cham 2015, 59.

74. Klemm, D.; Kramer, F.; Moritz, S.; Lindstrom, T.; Ankerfors, M.; Gray, D.; Dorris, A., Nanocelluloses: a new family of nature-based materials. Angewandte Chemie 2011, 50, 5438. 75. Tran, A.; Boott, C. E.; MacLachlan, M. J., Understanding the self-assembly of cellulose nanocrystals-toward chiral photonic materials. Advanced Materials 2020, 1905876. 
76. George, J.; Sabapathi, S. N., Cellulose nanocrystals: synthesis, functional properties, and applications. Nanotechnology, Science and Applications 2015, 8, 45.

77. Lagerwall, J. P. F.; Schütz, C.; Salajkova, M.; Noh, J.; Hyun Park, J.; Scalia, G.; Bergström, L., Cellulose nanocrystal-based materials: from liquid crystal self-assembly and glass formation to multifunctional thin films. $N P G$ Asia Materials 2014, 6, 80 .

78. Rofouie, P.; Alizadehgiashi, M.; Mundoor, H.; Smalyukh, I. I.; Kumacheva, E., Selfassembly of cellulose nanocrystals into semi-spherical photonic cholesteric films. Advanced Functional Materials 2018, 28, 1803852.

79. Fernandes, S. N.; Almeida, P. L.; Monge, N.; Aguirre, L. E.; Reis, D.; de Oliveira, C. L.; Neto, A. M.; Pieranski, P.; Godinho, M. H., Mind the microgap in iridescent cellulose nanocrystal films. Advanced Materials 2017, 29, 1603560.

80. Kose, O.; Boott, C. E.; Hamad, W. Y.; MacLachlan, M. J., Stimuli-responsive anisotropic materials based on unidirectional organization of cellulose nanocrystals in an elastomer. Macromolecules 2019, 52, 5317.

81. Siqueira, G.; Abdillahi, H.; Bras, J.; Dufresne, A., High reinforcing capability cellulose nanocrystals extracted from Syngonanthus nitens (Capim Dourado). Cellulose 2010, 17, 289.

82. Yang, J.; Han, C. R.; Duan, J. F.; Xu, F.; Sun, R. C., Mechanical and viscoelastic properties of cellulose nanocrystals reinforced poly(ethylene glycol) nanocomposite hydrogels. ACS Applied Materials \& Interfaces 2013, 5, 3199.

83. Wang, X.; Huang, H.; Liu, H.; Rehfeldt, F.; Wang, X.; Zhang, K., Multi-responsive bilayer hydrogel actuators with programmable and precisely tunable motions. Macromolecular Chemistry and Physics 2019, 220, 1800562.

84. Yang, J.; Xu, F.; Han, C. R., Metal ion mediated cellulose nanofibrils transient network in covalently cross-linked hydrogels: mechanistic insight into morphology and dynamics. Biomacromolecules 2017, 18, 1019.

85. Huang, H.; Wang, Y.; Wang, X.; Rehfeldt, F.; Zhang, K., Robust heterogeneous hydrogels with dynamic nanocrystal-polymer interface. Macromolecular Rapid Communications 2017, 38,1600810 .

86. Appel, E. A.; Tibbitt, M. W.; Greer, J. M.; Fenton, O. S.; Kreuels, K.; Anderson, D. G.; 
Langer, R., Exploiting electrostatic interactions in polymer-nanoparticle hydrogels. ACS Macro Letters 2015, 4, 848.

87. Oechsle, A.-L.; Lewis, L.; Hamad, W. Y.; Hatzikiriakos, S. G.; MacLachlan, M. J., $\mathrm{CO}_{2}-$ switchable cellulose nanocrystal hydrogels. Chemistry of Materials 2018, 30, 376.

88. Zubik, K.; Singhsa, P.; Wang, Y.; Manuspiya, H.; Narain, R., Thermo-responsive poly(Nisopropylacrylamide)-cellulose nanocrystals hybrid hydrogels for wound dressing. Polymers 2017, 9, 119.

89. Kelly, J. A.; Shukaliak, A. M.; Cheung, C. C.; Shopsowitz, K. E.; Hamad, W. Y.; MacLachlan, M. J., Responsive photonic hydrogels based on nanocrystalline cellulose. Angewandte Chemie 2013, 52, 8912.

90. Hiratani, T.; Kose, O.; Hamad, W. Y.; MacLachlan, M. J., Stable and sensitive stimuliresponsive anisotropic hydrogels for sensing ionic strength and pressure. Materials Horizons 2018, 5, 1076.

91. Huang, H.; Wang, X.; Yu, J.; Chen, Y.; Ji, H.; Zhang, Y.; Rehfeldt, F.; Wang, Y.; Zhang, K., Liquid-behaviors-assisted fabrication of multidimensional birefringent materials from dynamic hybrid hydrogels. ACS nano 2019, 13, 3867.

92. Duan, B.; Huang, Y.; Lu, A.; Zhang, L., Recent advances in chitin based materials constructed via physical methods. Progress in Polymer Science 2018, 82, 1.

93. Zeng, J. B.; He, Y. S.; Li, S. L.; Wang, Y. Z., Chitin whiskers: An overview. Biomacromolecules 2012, 13, 1.

94. Narkevicius, A.; Steiner, L. M.; Parker, R. M.; Ogawa, Y.; Frka-Petesic, B.; Vignolini, S., Controlling the Self-Assembly Behavior of Aqueous Chitin Nanocrystal Suspensions. Biomacromolecules 2019, 20, 2830.

95. Mincea, M.; Negrulescu, A.; Ostafe, V., Preparation, modification, and applications of chitin nanowhiskers: a review. Reviews on Advanced Materials Science 2012, 30, 225.

96. Mendoza-Galván, A.; Muñoz-Pineda, E.; Järrendahl, K.; Arwin, H., Birefringence of nanocrystalline chitin films studied by Mueller-matrix spectroscopic ellipsometry. Optical Materials Express 2016, 6, 671.

97. Liu, M.; Huang, J.; Luo, B.; Zhou, C., Tough and highly stretchable polyacrylamide 
nanocomposite hydrogels with chitin nanocrystals. International Journal of Biological Macromolecules 2015, 78, 23.

98. Wang, Q.; Chen, S.; Chen, D., Preparation and characterization of chitosan based injectable hydrogels enhanced by chitin nano-whiskers. Journal of the Mechanical Behavior of Biomedical Materials 2017, 65, 466.

99. Huang, Y.; Yao, M.; Zheng, X.; Liang, X.; Su, X.; Zhang, Y.; Lu, A.; Zhang, L., Effects of chitin whiskers on physical properties and osteoblast culture of alginate based nanocomposite hydrogels. Biomacromolecules 2015, 16, 3499.

100. Azuma, K.; Izumi, R.; Osaki, T.; Ifuku, S.; Morimoto, M.; Saimoto, H.; Minami, A.; Okamoto, Y., Chitin, chitosan, and its derivatives for wound healing: old and new materials. Journal of Functional Biomaterials 2015, 6, 104.

101. Liu, Y.; Liu, M.; Yang, S.; Luo, B.; Zhou, C., Liquid crystalline behaviors of chitin nanocrystals and their reinforcing effect on natural rubber. ACS Sustainable Chemistry \& Engineering 2018, 6, 325.

102. Le Corre D, Bras J, Dufresne A. Starch nanoparticles: a review. Biomacromolecules 2010, $11,1139$.

103. Tang, H.; Mitsunaga, T.; Kawamura, Y., Molecular arrangement in blocklets and starch granule architecture. Carbohydrate Polymers 2006, 63, 555.

104. A. Donald, P. J., T. Waigh, Council for the Central Laboratory of the Research Councils 1997, 54.

105. Gallant, D. J.; Bouchet, B.; Baldwin, P. M., Microscopy of starch: evidence of a new level of granule organization. Carbohydrate Polymers 1997, 32, 177.

106. Le Corre, D.; Angellier-Coussy, H., Preparation and application of starch nanoparticles for nanocomposites: A review. Reactive and Functional Polymers 2014, 85, 97.

107. Lin, N.; Huang, J.; Dufresne, A., Preparation, properties and applications of polysaccharide nanocrystals in advanced functional nanomaterials: a review. Nanoscale 2012, 4, 3274 .

108. Dufresne, A., Starch and nanoparticle. In Polysaccharides: Bioactivity and Biotechnology, Ramawat, K. G.; Mérillon, J.-M., Eds. Springer International Publishing: Cham, 2015, 417. 
109. Bakrudeen, H. B.; Sudarvizhi, C.; Reddy, B. S. R., Starch nanocrystals based hydrogel: construction, characterizations and transdermal application. Materials Science and Engineering C-Materials for Biological Applications 2016, 68, 880.

110.Piluso, S.; Labet, M.; Zhou, C.; Seo, J. W.; Thielemans, W.; Patterson, J., Engineered threedimensional microenvironments with starch nanocrystals as cell-instructive materials. Biomacromolecules 2019, 20, 3819. 


\section{Appendix}

Author's peer-reviewed publications 


\section{Publication 1}

\section{Multi-responsive bilayer hydrogel actuators with programmable and}

\section{precisely tunable motions}

Xiaojie Wang ${ }^{1}$, Heqin Huang ${ }^{1}$, Huan Liu ${ }^{1}$, Florian Rehfeldt ${ }^{2}$, Xiaohui Wang ${ }^{3}$ and Kai Zhang ${ }^{*}, 1$

${ }^{1}$ Wood Technology and Wood Chemistry, Georg-August-University of Göttingen, Büsgenweg 4, D-37077 Göttingen, Germany

${ }^{2}$ Third Institute of Physics - Biophysics, Faculty of Physics, Georg-August-University of Göttingen, Friedrich-Hund-Platz 1, D-37077 Göttingen, Germany

${ }^{3}$ State Key Laboratory of Pulp and Paper Engineering, South China University of Technology, Guangzhou, 510640, China

*Tel.: +49 551 394505; E-mail: kzhang1@uni-goettingen.de

Originally published in:

Macromolecular Chemistry and Physics

John Wiley \& Sons

DOI: $10.1002 /$ macp.201800562

Received: December 19, 2018; Published online: February 7, 2019 
Abstract: Multi-responsive hydrogel actuators show promising applications for soft robotics, biomedical engineering and artificial muscles, but the uncontrollable nature of their motions poses a barrier to practical applications. Herein, we presented a novel type of bilayer hydrogel actuators (BHAs) comprising of a poly(N-isopropylacrylamide) (PNIPAm) and a poly (Nhydroxyethyl acrylamide) (PHEAm) hydrogel layer with various compositions, which demonstrated thermal-responsive and novel solvent-responsive actuation under water or within solvents. These BHAs exhibited a wide range of regulable bidirectional motions due to the simultaneous co-nonsolvency property of PNIPAm and the shrinking behavior of PHEAm in ethanol/water mixtures with various ethanol contents. By adjusting the compositions of ethanol/water mixtures, the bending directions and amplitudes of BHAs were precisely regulable and the curvatures of actuators were tunable between -0.34 and 0.3 . Because of temperature-responsive character of PNIPAm, BHAs fulfilled thermal-driven motions to lift items. By reinforcing PHEAm layers with cellulose nanocrystals (CNCs) or CNCs bearing methacylamide moieties on surface (CNCs-MAm), the weight-lifting capability of BHAs was highly improved to 18 times the weight of their own polymer weights. This design concept with bilayer structures provides a new strategy for the construction of precisely regulable hydrogel actuators, which allows using solvents to exactly control their motions.

The flourishing development of versatile stimuli-responsive polymers leads to the emergence of flexible polymer actuators. ${ }^{[1,2]}$ Hydrogels are highly hydrated three-dimensional polymer networks, which showed excellent biodegradability, biocompatibility and permeability. ${ }^{[3-5]}$ In recent years, hydrogel-based actuators that can undergo precisely tunable motions enable transformative applications in diverse areas, such as biomedical fields, ${ }^{[6,7]}$ soft robotics $^{[8-10]}$ and soft electronics. ${ }^{[1-13]}$ Typically, actuations of existing responsive hydrogels mostly rely on their swelling and de-swelling in response to external stimuli such as temperature, ${ }^{[14,15]} \mathrm{pH},{ }^{[16,17]}$ electric field, ${ }^{[18]}$ light ${ }^{[19,20]}$ and ionic strength. ${ }^{[21]}$ Mostly, these systems utilize the volume phase transition and fulfill the shape alteration between different states. ${ }^{[22]}$ So far, hydrogels featuring many other fascinating properties were continuously appearing and all these versatile hydrogels 
were constituted the building blocks for the construction of advanced hydrogel actuators. ${ }^{[23-25]}$ $\mathrm{Hu}$ et al. reported highly anisotropic and ion-conductive wood based hydrogels with high tensile strength of $36 \mathrm{MPa}$ along the longitudinal direction. ${ }^{[26]}$ By utilizing the electrical field, ${ }^{[27]}$ magnetic field ${ }^{[28]}$ and other advanced engineering technologies including the $3 \mathrm{D}$ printing ${ }^{[29]}$ and electrospinning, ${ }^{[30]}$ hydrogel actuators have got great improvement in motion amplitude and response speed. ${ }^{[31]}$ At the same time, many efforts have been devoted to program the movement directions and get various shapes at end state. Yet, most of these methods require multi-control systems, large-scale equipment and complex preparation. More importantly, only a few hydrogel actuators can perform bidirectional motions with tunable amplitude, such as the polyelectrolyte hydrogels. ${ }^{[32]}$ However, most of hydrogel actuators can only switch between the starting and end point, while they are difficult to halt at any certain state in between and their behaviors are not precisely controllable. These existing drawbacks pose currently huge barrier to the development of hydrogel actuators and their application under complex surroundings, such as under water.

In this work, we fabricated thermal- and solvent-responsive BHAs with programmable and controllable bending motions and amplitudes. The BHAs were comprised of PNIPAm as the active layer and PHEAm without or with various reinforcing CNCs as the cooperating layer. The reversible phase transition of PNIPAm in response to temperature endowed the BHAs with thermal-driven behaviors. Furthermore, solvent-responsive properties of BHAs for the precise control over their actuation were investigated in detail. The co-nonsolvency property of PNIPAm can swell and deswell in water/ethanol mixture depending on the ratio of both solvents, while various PHEAm hydrogels shrink more strongly with increasing ethanol content. These two different mechanisms endowed the BHAs with the feasibility to perform a wide range of precisely controllable motions in tunable directions. With bare CNCs or crosslinkable CNCs bearing methacrylamide moieties on surface in PHEAm layers, the weightlifting capacity of BHAs was tuned to 14 and 18 times the weight of their own polymer weights, respectively. Moreover, their motions can be programmed to stop with desired amplitudes at any position by gradually adjusting the compositions of ethanol/water mixtures. 
a

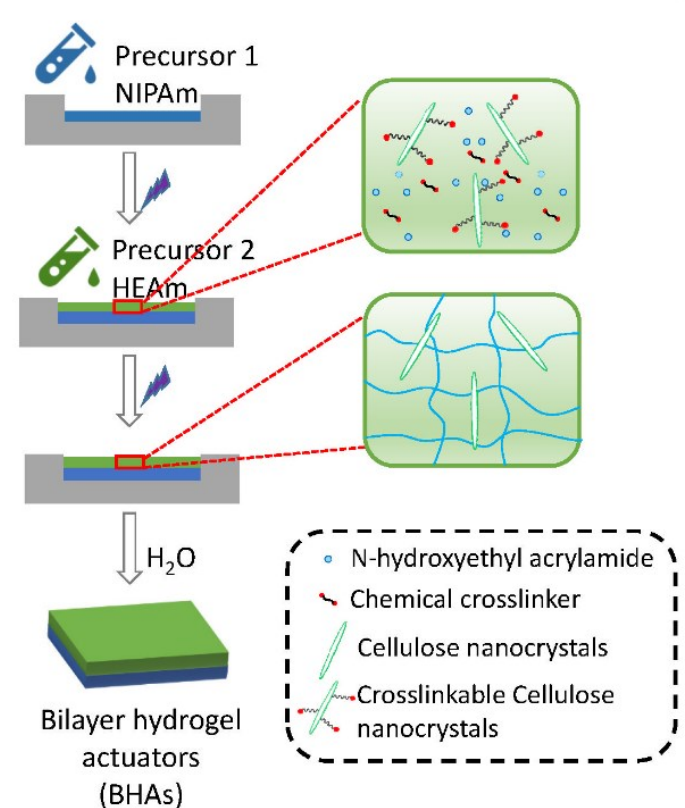

b

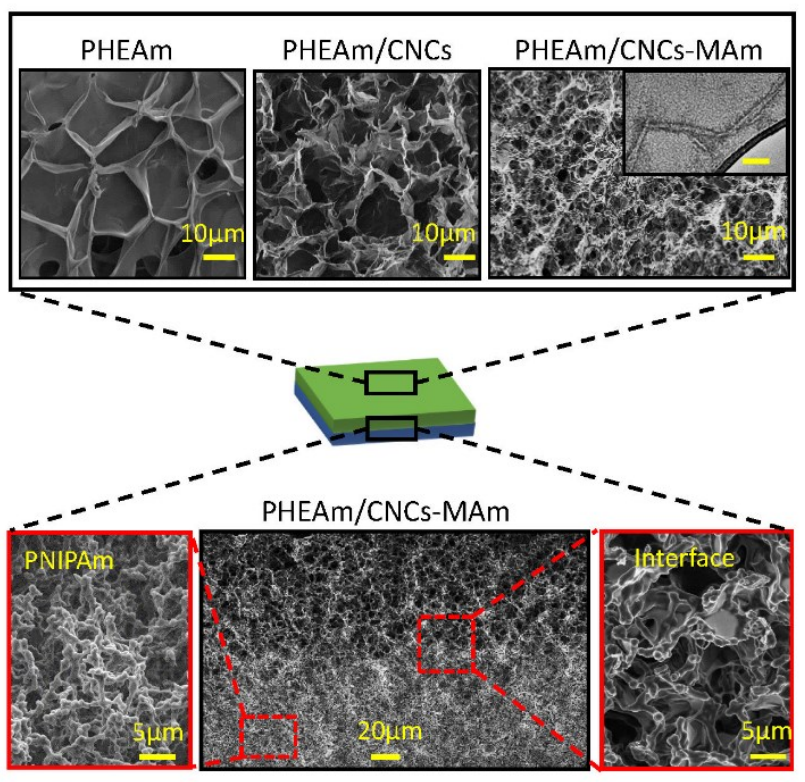

Figure 1. Schematic illustration for the preparation and the microstructures of BHAs. a) The preparation of BHAs. b) The microstructures of each layer and the interface of the BHAs. The inset showed the TEM image of CNCs-MAm with the scale bar of $50 \mathrm{~nm}$.

The BHAs were prepared via a two-step method. Equal PNIPAm hydrogels in all BHAs were prepared after the polymerization of NIPAm with chemical crosslinkers at first as the active layers. Then, various PHEAm layers were fabricated above the PNIPAm layer as the cooperating layers. BHAs were obtained after equilibrated in deionized water (DI water) (Figure 1a). PHEAm hydrogels in diverse BHAs were prepared with three different compositions: bulk crosslinked PHEAm in BHAs/P, PHEAm networks containing reinforcing CNCs (PHEAm/CNCs) in BHAs/CNCs, and PHEAm networks containing crosslinkable CNCs-MAm (PHEAm/CNCs-MAm) in BHAs/CNCs-MAm.

CNCs were prepared via the TEMPO-mediated oxidation with $1.37 \pm 0.2 \mathrm{mmol} \mathrm{g}^{-1}$ carboxyl groups on the surface (Figure S1, Supporting Information). CNCs-MAm were synthesized after the reaction of methacrylic anhydride with $\mathrm{CNCs}-\mathrm{NH}_{2}$, which were obtained via the amidation reaction of CNCs with hexamethylenediamine. The amount of methyl acrylamide groups on CNCs-MAm was measured to be $0.5 \pm 0.05 \mathrm{mmol} \mathrm{g}^{-1}$ (Table S1, Supporting Information). 
CNCs-MAm have dimensions of $\sim 200 \mathrm{~nm} \times 20 \mathrm{~nm}$ (length $\times$ width) (Figure $1 \mathrm{~b}$ ).

The scanning electron microscopy (SEM) images in Figure $1 \mathrm{~b}$ clearly showed the distinguishable PHEAm/CNCs-MAm layer and PNIPAm layer with different pore topologies within BHAs after freeze-drying. A much denser area is visible at the interface of the two layers, indicating the interpenetration of two kinds of polymer chains in this interfacial area. This dense interface in turn enhanced the connection of the two hydrogel layers. As the result, the covalently crosslinked interactions tightly fix the two hydrogel layers together, which were sufficient to tolerate the swelling and actuation behaviors triggered by various stimuli. Furthermore, the introduction of CNCs and crosslinkable CNCs-MAm led to much smaller pore size for the cooperating hydrogel layers. The average pore size of the freeze-dried PHEAm/CNCs layer was around $12 \pm 3 \mu \mathrm{m}$, while even smaller pores of averagely $770 \pm 200$ $\mathrm{nm}$ were observed in the PHEAm/CNCs-MAm layer (Figure 1b).

a

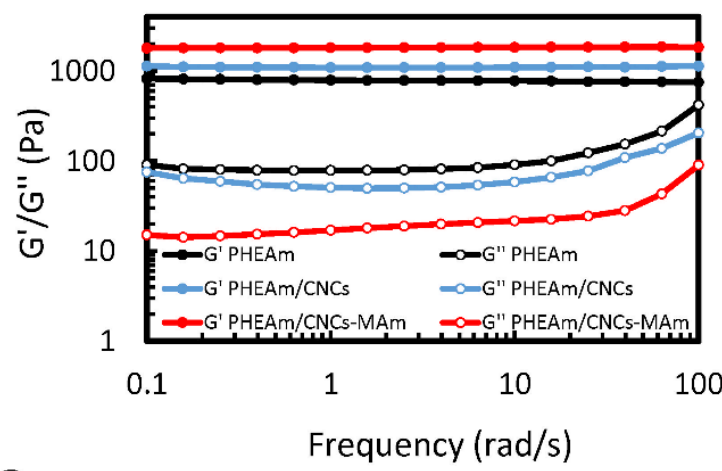

C

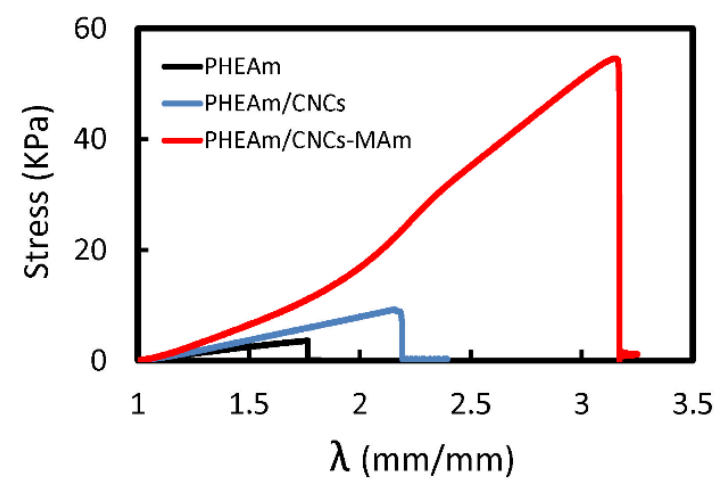

b

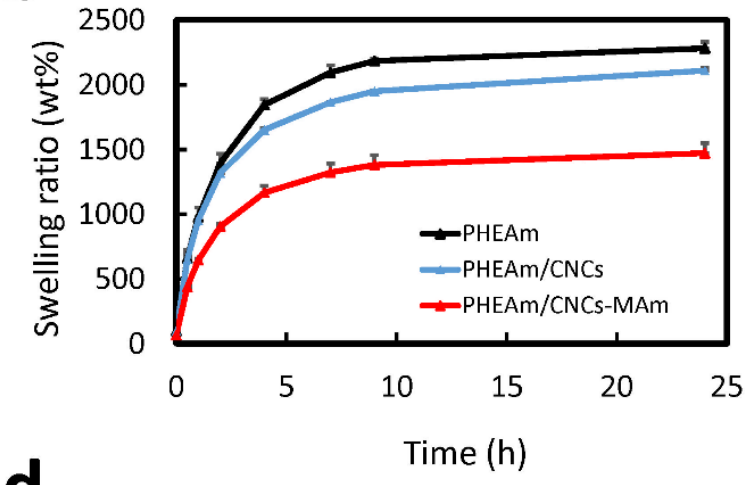

d

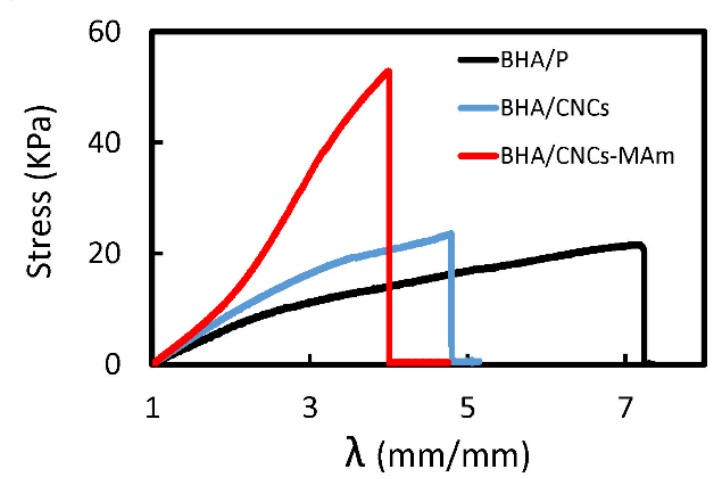

Figure 2. Mechanical properties and swelling behaviors of the cooperating layers and BHAs.

a) The frequency sweep rheology tests of the cooperating PHEAm layers. b) The swelling ratios 
of the cooperating PHEAm layers. c) Representative tensile test curves of the cooperating PHEAm layers. d) Representative tensile test curves of BHAs.

The mechanical properties of various BHAs with distinct compositions were further characterized. In particular, the mechanical properties of cooperating PHEAm layers based on PHEAm with various compositions were characterized due to their distinct microstructures, while the active PNIPAm layers maintained equal in all BHAs (Figure 1b). The frequency sweep rheology tests were performed at first on diverse PHEAm hydrogels (Figure 2a). Both storage modulus (G') and loss modulus (G”) of various PHEAm hydrogels exhibited similar frequency-independency over the whole frequency range, indicating the covalent crosslinking networks within these hydrogels. Furthermore, the swelling behaviors of the cooperating PHEAm hydrogel layers also showed that the presence of CNCs and in particular crosslinkable CNCs-MAm resulted in smaller swelling ratios compared to bulk PHEAm hydrogels (Figure 2b). This should be due to the denser crosslinking networks caused by CNCs and CNCs-MAm, which resisted the water absorption.

Accompanying with the denser hydrogel matrix, CNCs and CNCs-MAm improved the stiffness of hydrogels with diverse extents. While CNCs only acted as neutral nanofillers and showed limited enhancement on the breaking stress of resulting hydrogels to about 2.5 times of PHEAm hydrogels, crosslinkable CNCs-MAm efficiently increased the breaking stress of obtained hydrogels to roughly 14 times of PHEAm hydrogels. Furthermore, the tensile tests clearly displayed the effective toughening by introducing CNCs and CNCs-MAm into the PHEAm hydrogels (Figure 2c). The PHEAm/CNCs-MAm showed highly enhanced elastic modulus, which was up to 2.6 times of the elastic modulus of PHEAm hydrogels. The elongation of PHEAm/CNCs-MAm was also improved to 1.8 times of the elongation of PHEAm hydrogels. This enhancement showed in both as-prepared state and also, even with further amplification, swollen state (Figure S2 and S3, Supporting Information). Benefiting from the extra crosslinking with CNCs-MAm, the PHEAm/CNCs-MAm hydrogels also showed clear strain-induced hardening during the stretching. This phenomenon was in agreement with the tensile curves provided by the typical hydrogels containing 
nanocrosslinkers. ${ }^{[33]}$ In addition, this reinforcing effect by CNCs and CNCs-MAm was still maintained in the BHAs. As shown in Figure 2d, the mechanical properties of BHAs containing CNCs and CNCs-MAm were largely improved only by the reinforcement in the cooperating PHEAm hydrogel layers. On the contrary, these reinforced PHEAm hydrogels showed much smaller elongation ratios in comparison to bulk PHEAm hydrogels, which resulted in reduced elongation for BHAs/CNCs (68\%) and BHAs/CNCs-MAm (56\%) compared to BHAs/P. The elongation of BHAs significantly decreased with increasing stress, because the comparatively brittle PNIPAm layer cracked fast during stretching and induced defects to BHAs.

a

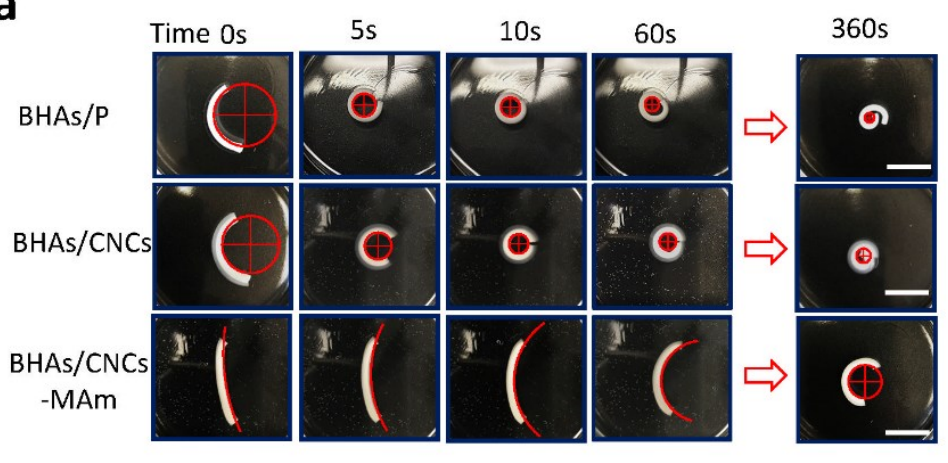

C

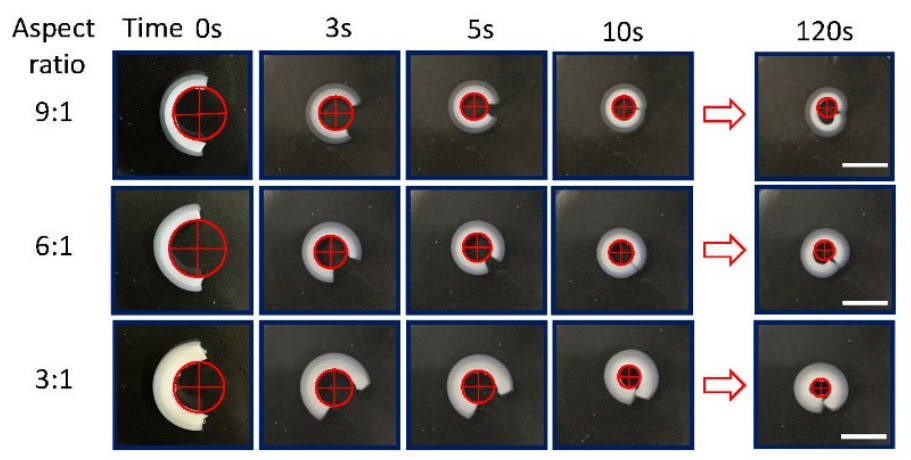

b

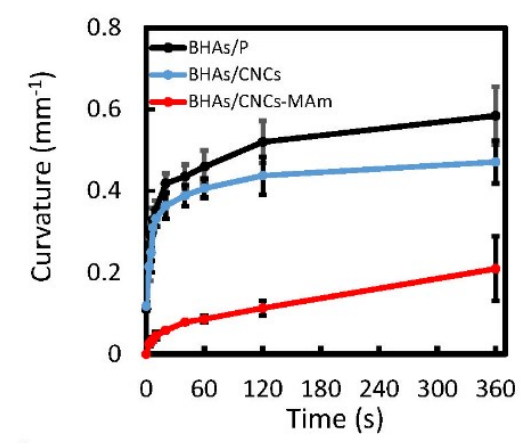

d

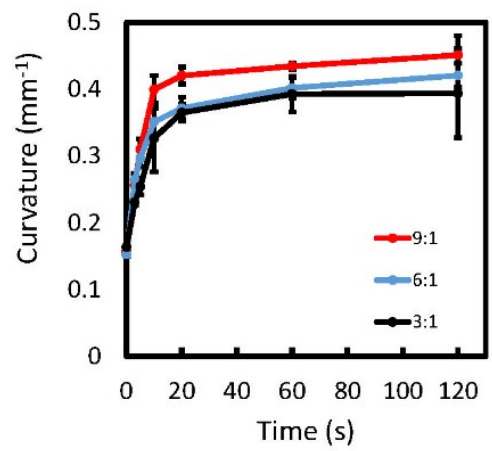

Figure 3. Dynamic thermal behaviors of BHAs in water of $40^{\circ} \mathrm{C}$. a) Optical photographs of the dynamic actuation process of the BHAs with diverse cooperating layers (scale bars: $1 \mathrm{~cm}$ ); b) Dynamic changes of the curvature values of BHAs/P, BHAs/CNCs and BHAs/CNCs-MAm at $40{ }^{\circ} \mathrm{C}$; c) Optical photographs of the dynamic actuation process of and d) Dynamic changes of the curvature values of BHAs/CNCs with various aspect ratios of 9:1, 6:1 and 3:1 at $40{ }^{\circ} \mathrm{C}$. Scale bars in c: $1 \mathrm{~cm}$. 
Given the great difference in mechanical properties caused by the introduction of CNCs and CNCs-MAm in cooperating layers, we further studied the thermal behaviors of BHAs containing diverse cooperating layers and with various aspect ratios (length:width). PNIPAm has a LCST at about $32{ }^{\circ} \mathrm{C}$ and exhibits greater hydrophobicity at the temperature above its LCST. ${ }^{[34]}$ When BHAs were immersed into water of $40{ }^{\circ} \mathrm{C}$, PNIPAm chains aggregated which induced shrinkage of the PNIPAm layers and all BHAs bended to the PNIPAm side (Figure 3a). The magnitudes of their dynamic bending motions were illustrated by the corresponding curvatures of BHAs after a certain time. It is obvious that BHAs containing diverse compositions showed distinct bending amplitudes according to their curvatures after the same bending times during the dynamic process (Figure $3 \mathrm{a}$ and $3 \mathrm{~b}$ ).

Generally, the continuous bending completed within 6 min and the shapes of the BHAs at 6 min were set as the final state. The BHAs/CNCs-MAm exhibited the smallest motion range and the curvature lay between 0.018-0.2, while BHAs/P showed the largest bending range with the curvature roughly between 0.11-0.6. The curvatures of BHAs/CNCs during the bending changed from 0.12 to 0.47 (Figure $3 \mathrm{a}$ and $3 \mathrm{~b}$ ). Because of the equal PNIPAm layers, this difference should be mainly attributed to the different mechanical properties of the cooperating layers. This different performance in motion magnitude can be explained using the simplified Timoshenko equation (Equations (1); Equation (S3), Supporting Information).

$$
k=\frac{24 \alpha_{n}}{h\left(14+y+\frac{1}{y}\right)}
$$

where $\mathrm{k}$ represents the curvature which were defined as $\mathrm{k}_{1}(\mathrm{BHAs} / \mathrm{P}), \mathrm{k}_{2}(\mathrm{BHAs} / \mathrm{CNCs})$ and $\mathrm{k}_{3}$ (BHAs/CNCs-MAm), y is the ratio of their Young's moduli $y=E c / E n$, the subscript $\mathrm{n}$ represents the PNIPAm layer and c represents the various PHEAm layers.

Comparing various BHAs, the introduction of CNCs and CNCs-MAm increased the Young's modulus of the cooperating layers (Figure S3, Supporting Information), resulting in $\mathrm{y}_{1}<\mathrm{y}_{2}<\mathrm{y}_{3}$. As the result, stronger cooperating layers induced relatively smaller curvatures, thus $\mathrm{k}_{1}>\mathrm{k}_{2}>\mathrm{k}_{3}$. 
Furthermore, we studied the influence of the aspect ratios on the actuation behaviors of BHAs with BHAs/CNCs as the models. Since the fast actuation behavior is preferred for actuators, we focused on the first 120 seconds. The actuation processes of BHAs/CNCs with three different aspect ratios (length:width of 9:1, 6:1 and 3:1 with fixed length of $2.7 \mathrm{~cm}$ ) were analyzed (Figure 3c). At the aspect ratio of 9:1, the actuator strips showed the fastest bending motion until the final state, while BHAs/CNCs with the aspect ratio of 3:1 exhibited the lowest bending speed. Nevertheless, all BHAs/CNCs demonstrated independent on the aspect ratio similar bending curvatures after $120 \mathrm{~s}$ (Figure 3d). Because all these BHAs/CNCs shared same length and the same thickness with changing widths, their only difference is their volumes. Thus, these diverse bending speeds of BHAs/CNCs with distinct aspect ratios should be attributed to their different volumes, which required distinct times for the thermal conduction to reach similar temperature through the whole BHAs. This is in particular essential for the bending at the beginning. After sufficient time for the thermal conduction, all samples still reached the same bending curvature.
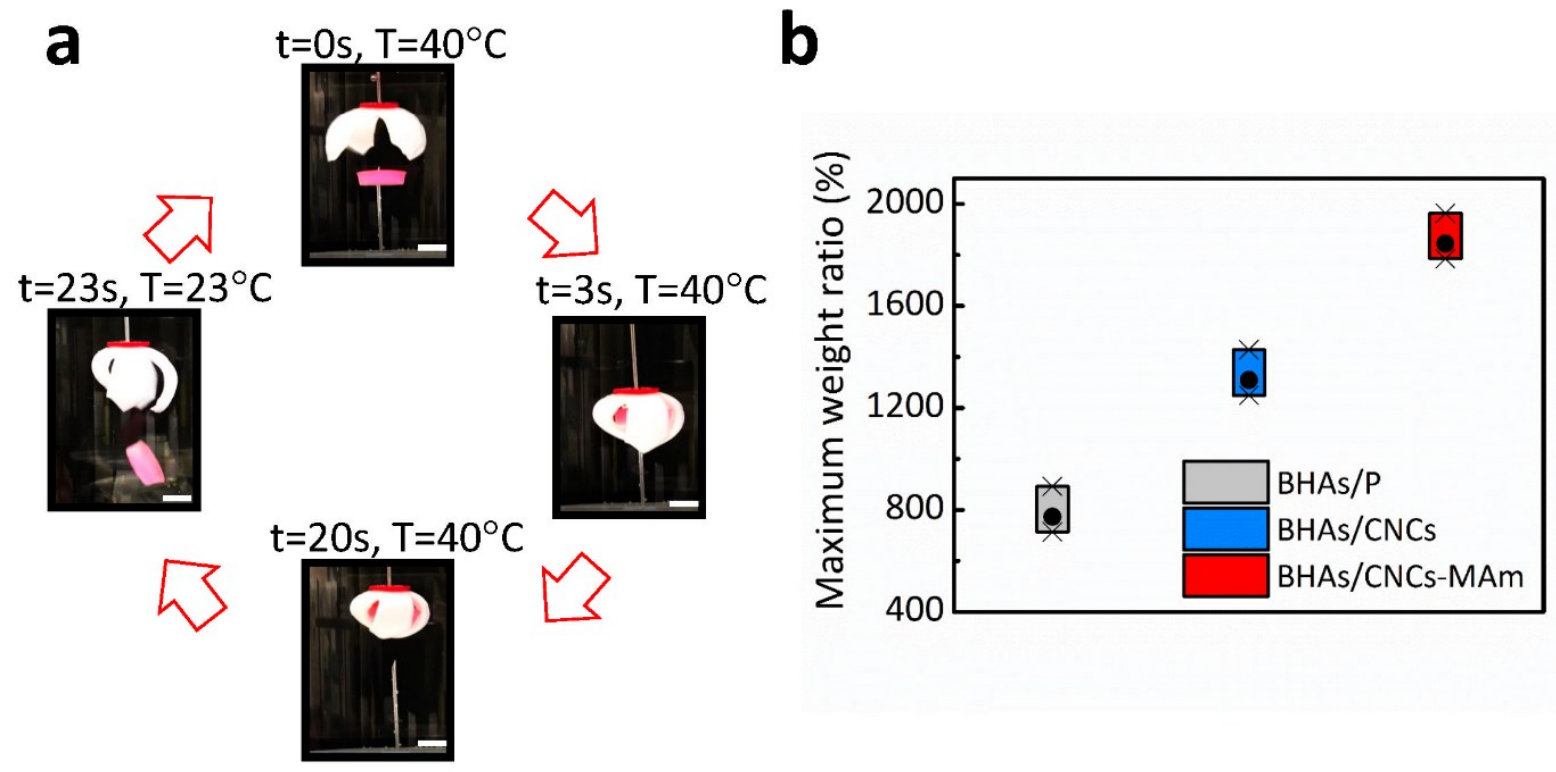

Figure 4. Manipulation of thermal-responsive BHAs. a) Grasp and release of target object using BHAs/CNCs as example (scale bars: $1 \mathrm{~cm}$ ). b) The maximum weight ratios for various BHAs (maximum weight ratios $=$ maximum weights that actuators can lift/own polymer 
weights).

Moreover, these relatively fast thermal actuations of BHAs ensured them the potential to be used as soft robotics. These BHAs can be manipulated by changing the environmental temperature (Figure 4, Movie S1 and S2, Supporting Information). They were prepared as grippers and were designed to mimic the grasping and releasing actions of human hands in DI water (Figure 4a). The BHAs grippers were fully expanded in water at $23{ }^{\circ} \mathrm{C}$, and grabbed a target object at $40{ }^{\circ} \mathrm{C}$. The grabbed object was released in the $23{ }^{\circ} \mathrm{C}$ water bath. With the reinforcement of the cooperating PHEAm layers with CNCs or CNCs-MAm, the grabbing capabilities of BHAs were also increased (Figure 4b). BHAs/CNCs-MAm showed the highest maximum weight ratio and could lift items that were about 18 times the weight of own polymer weight. While the BHAs/CNCs could hold items of roughly $1400 \mathrm{wt} \%$ of own polymer weight, and BHAs containing PHEAm only could lift objects of $800 \mathrm{wt} \%$ of own polymer weight. Therefore, stronger cooperating layers highly promoted the loading capability of BHAs, independent on the active PNIPAm layers.

This kind of thermal triggered actuator is a traditional driven mode which can be stimulated by external triggers to bend until to its final state. However, the uncontrollable motion amplitude restricts its practical applications. 

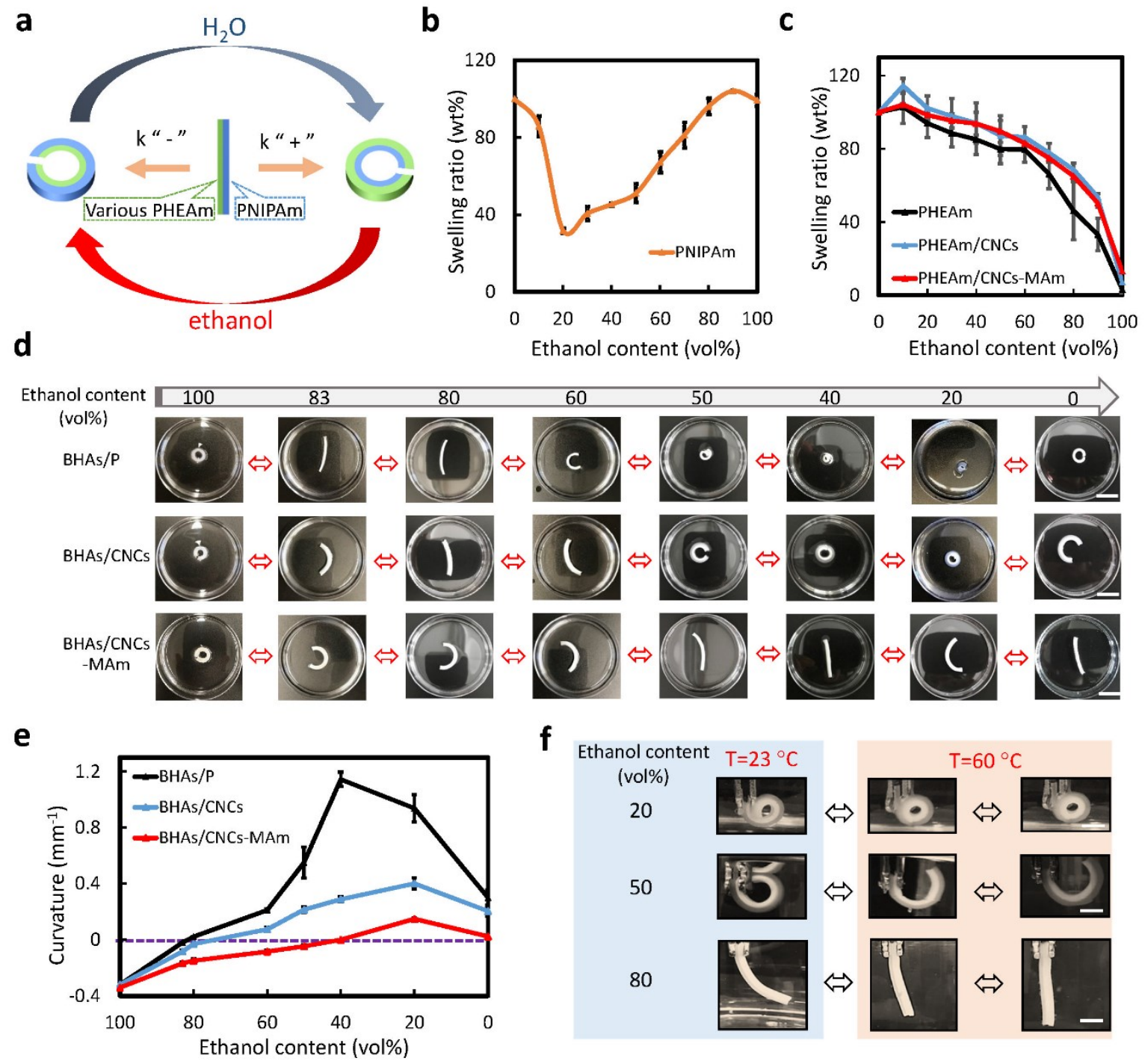

Figure 5. Dynamic behaviors of BHAs triggered by ethanol. All BHAs were constituted by the PNIPAm layers on the right side and various PHEAm layers on the left side. a) Schematic illustration for the bidirectional bending motions actuated by the solvent compositions. The $\mathrm{k}$ represents the bending curvature with the bending to PNIPAm side recorded as "+" and the bending to PHEAm layers marked as “-”. b) The swelling ratios of PNIPAm hydrogels in various ethanol/water mixtures. c) The swelling ratios of various PHEAm hydrogels in various ethanol/water mixtures. d) Photos of actuation processes of three BHAs (scale bars: $10 \mathrm{~mm}$ ). e) Dynamic changes of the curvatures of BHAs during the actuation process. f) Reversed thermal-responsive behaviors of $\mathrm{BHAs} / \mathrm{CNCs}$ in ethanol/water mixtures with the ethanol contents of $20 \%, 50 \%$ and $80 \%$ (scale bars: $7 \mathrm{~mm}$ ). 
In addition to this thermal actuation performance, the BHAs we reported here managed to bend with controllable motion amplitude which can be programmed to stop at certain amplitude. The BHAs exhibited unique solvent-responsive properties in ethanol/water mixtures (Figure 5a). The co-nonsolvency property of PNIPAm in ethanol/water mixtures endows PNIPAm hydrogels with the ability to swell and deswell depending on the ratio of both solvents. At the same time, various PHEAm cooperating layers shrink more strongly with increasing ethanol content. Nevertheless, these two different behaviors of the PNIPAm and PHEAm hydrogel layers in the ethanol/water mixtures synergistically contributed to extraordinary solventresponsive behaviors of our BHAs. Subsequently, this co-solvent actuation not only generated bidirectional bending motions, the bending amplitudes (represented by their corresponding curvatures k) were also adjustable by changing the solvent composition and the BHAs could be fixed at a certain bending state (Figure 5a-5d).

We investigated at first the mechanism of solvent-triggered actuation. The swelling/shrinking behaviors of PNIPAm and PHEAm in ethanol/water mixtures of various ratios were analyzed (Figure $5 \mathrm{~b}$ and $5 \mathrm{c}$ ). PNIPAm chains in the mixed solvents of water and ethanol experienced a coil-to-globule-to-coil transition, which is primarily attributed to the competitive formation of hydrogen bonding by water and ethanol molecules with polymer chains. ${ }^{[35]}$ Similar phenomenon also exists in our bulk crosslinked PNIPAm hydrogels (Figure 5b). With increasing ethanol content to the ethanol/water mixtures, the PNIPAm deswelled to the maximum at ethanol content 20 vol\%. Then, PNIPAm hydrogels slowly swelled with continuously increasing ethanol content, until the swelling ratios reached the maximum at the ethanol content of $90 \mathrm{vol} \%-100 \mathrm{vol} \%$. Therefore, it is obvious that the PNIPAm hydrogels underwent an alternative swelling-deswelling-swelling behavior with steady changing ethanol content.

In comparison, PHEAm hydrogels with various compositions exhibited continuous deswelling behaviors with increasing ethanol content in the ethanol/water mixtures (Figure 5c) which results in the shrinkage of the PHEAm hydrogels in the macro view.

Based on these unique swelling/deswelling behaviors of both PNIPAm and PHEAm layers of 
BHAs in ethanol/water mixtures, ethanol/water mixtures with various compositions were used for the actuation (Figure 5d). The bending motions of BHAs were continuously modulated during the alteration of ethanol/water ratios, as shown by changing the ethanol content between 0-100 vol\%. BHAs were immersed at first into pure ethanol (with the ethanol content of 100 vol\%) to reach the equilibrium state. All BHAs bended towards the cooperating PHEAm layers and eventually formed a circle shape (Figure 5d). By gradually adding DI water to decrease the ethanol content, the BHAs successively bended back to the PNIPAm side depending on the ethanol content. In particular, the BHAs can be exactly fixed at a desired state according to the ethanol content. The bending curvatures of BHAs were measured to illustrate their precise solvent-responsive motions (Figure 5e). Furthermore, it is obvious that the compositions and thus the mechanical properties of the cooperating PHEAm layers significantly affected the performance of these diverse BHAs (Figure 5d and 5e).

During the process of lowering ethanol contents from 100 vol\%, the curvature of the BHAs increased from -0.32 to 1.14 and then decreased to 0.3 in the pure water. It should be noted that the bending curvature of BHAs with the bending to PNIPAm side is recorded as "+" and the bending to PHEAm layers as "-". When the ethanol content was roughly 82 vol\%, the BHAs stayed almost straight and the curvature was nearly 0 . In comparison, the bending ranges of BHAs/CNCs and BHAs/CNCs-MAm were much narrower. The curvatures of BHAs/CNCs changed from -0.32 to 0.4 and reached 0.2 in pure water, while BHA/CNCs-MAm showed the smallest bending range with the curvature between -0.34 and 0.03 . The ethanol content for the straight state occurred at $\sim 75$ vol $\%$ for BHAs/CNCs and at $\sim 40$ vol $\%$ for BHA/CNCs-MAm. Moreover, these ethanol-triggered bending motions can achieve continuous movements by continuously changing the ethanol content. Compared with thermal actuation, this actuation triggered by ethanol/water mixtures can generate bidirectional motions of BHAs with large and programmable motion amplitudes. More importantly, the bending extent and direction of BHAs can be programmed to stop at any certain state and be exactly controlled by adjusting the ethanol content. By now, the hydrogel actuators with bidirectional and large programmable motion amplitudes with exact control over the bending were very rarely reported. ${ }^{[32]}$ 
In addition, BHAs demonstrated a special reversed thermal-responsive behavior in ethanol/water mixtures at $60{ }^{\circ} \mathrm{C}$, as shown on BHAs/CNCs in three different ethanol/water mixtures. BHAs/CNCs were equilibrated in ethanol/water mixtures with ethanol content of 20 vol $\%, 50$ vol $\%$ and 80 vol $\%$, before the temperature was elevated to $60{ }^{\circ} \mathrm{C}$ (Figure $5 \mathrm{f}$ ). With the ethanol contents of $50 \mathrm{vol} \%$ and $80 \mathrm{vol} \%$, the BHAs/CNCs bended towards the cooperating layers, which was opposite from thermal-driven bending in pure water as shown above. In comparison, the BHAs/CNCs stayed straight at ethanol content $20 \mathrm{vol} \%$. This behavior should be ascribed to the modified upper critical solution temperature (UCST) of PNIPAm, which decreases with increasing ethanol content. ${ }^{[36]}$ The UCST of PNIPAm was lower than $60{ }^{\circ} \mathrm{C}$ within the ethanol/water mixtures with high ethanol contents. Above the UCST, the PNIPAm chains tried to recover their homogeneity from phase separation. Therefore, the BHAs/CNCs showed a reversed thermal-responsive behavior within the mixtures with ethanol contents of $50 \mathrm{vol} \%$ and $80 \mathrm{vol} \%$. In comparison, within the mixtures with a lower ethanol content, for instance of $20 \mathrm{vol} \%$, the temperature of $60{ }^{\circ} \mathrm{C}$ is higher than LCST but lower than the UCST of PNIPAm. Thus, the BHAs/CNCs did not show significant reversed thermal-responsive behaviors within these ethanol/water mixtures.
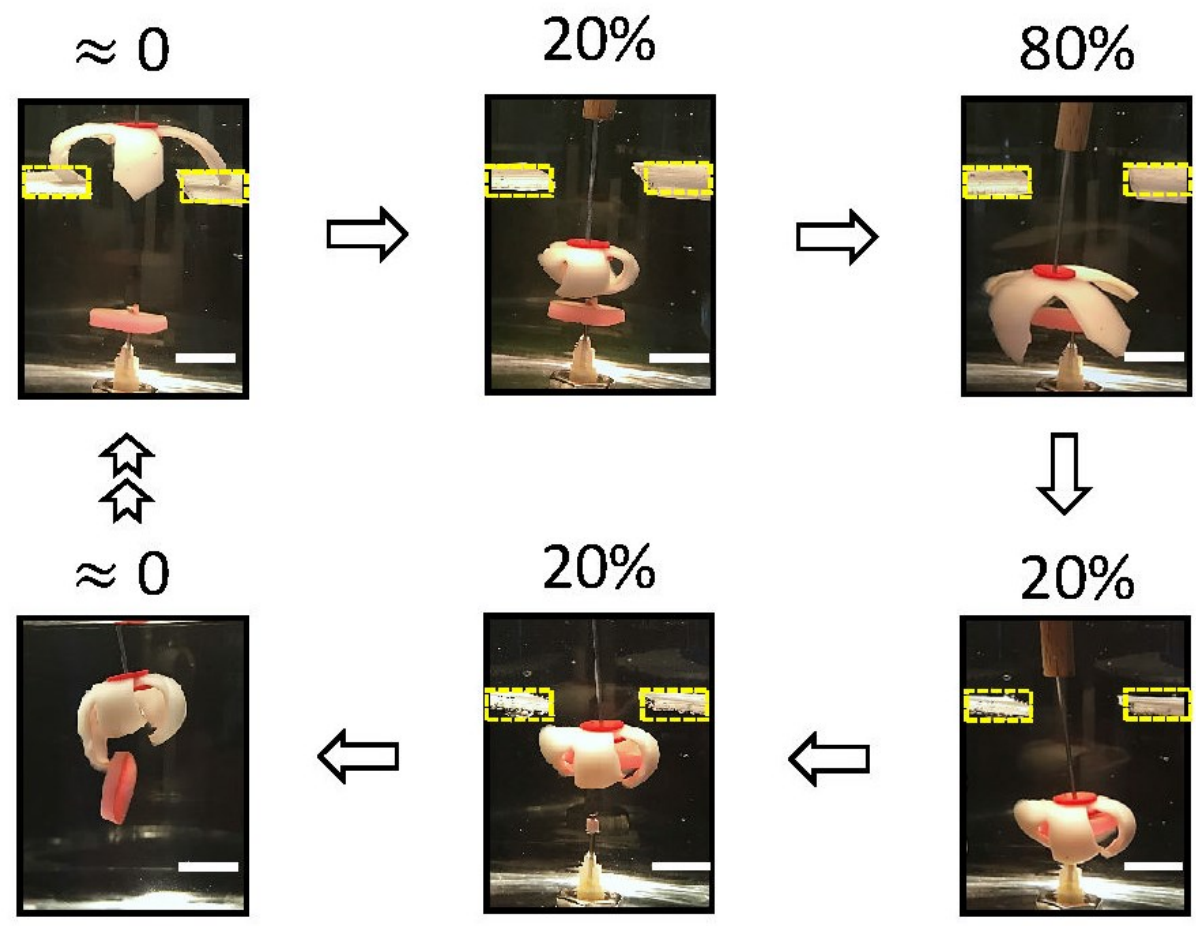
Figure 6. The dynamic behaviors of the BHAs/CNCs. The numbers represent the ethanol volume fraction in the ethanol/water mixtures. Optical photographs of the grabbing process of the BHAs/CNCs passing a narrow gate via regulating the ethanol contents. Yellow dash area indicates the narrow gate (Width of the gate is about $3.3 \mathrm{~cm}$ ). Scale bars: $2 \mathrm{~cm}$.

Thus, this ethanol-triggered actuation allows BHAs to autonomously change their bending direction and amplitudes according to the ethanol content in surrounding environment. Moreover, artificial grippers based on such BHAs can be used in complex conditions, such as to transport items through gates (Figure. 6). As showed on the gripper of BHAs/CNCs, the gripper in their smallest size easily passed the narrow gate within the ethanol/water mixture with $20 \mathrm{vol} \%$ ethanol. By increasing the ethanol content to $80 \mathrm{vol} \%$, the gripper expanded and could grab a target object. By diluting the solvent to ethanol content of $20 \mathrm{vol} \%$, the gripper easily grabbed the object and took it through the narrow gate. Eventually, this object was released in another surrounding as designed, e.g. in pure water. Thus, such BHAs showed promising practical applications in diverse fields.

In summary, we reported here a series of bilayer hydrogel actuators composed of various PHEAm hydrogels as cooperating layers and PNIPAm hydrogels as active layers. These BHAs demonstrated advantageous controllable thermal-responsive and solvent-driven actuation performance. The incorporation of strengthening CNCs and CNCs-MAm in the cooperative PHEAm layers effectively improved the loading capacities of BHAs. The grabbed weight was promoted from $800 \mathrm{wt} \%$ to $1800 \mathrm{wt} \%$ of their own polymer weight, although the introduction of CNCs and CNCs-MAm reduced motion amplitude and response speed.

The thermal-responsive property allows BHAs to bend responding to the surrounding temperature, while the ethanol-driven actuation provides the feasibility to exactly tune the bending amplitude and bending direction of BHAs by adjusting the ethanol content. Therefore, these BHAs exhibited in fact the bidirectional actuation, which can be continuously altered by modifying the compositions of ethanol/water mixtures. Thus, these pre-programmable motions and the feasibility to spontaneously freeze the state of BHAs demonstrate their great peculiarity. 
Based on these advantageous properties, other stimuli-responsive materials with other functions can be simply used as the cooperating layer, which provides such BHAs vast potential for real-world applications at different scales, such as soft robotics, drug delivery, and micro/nanoelectromechanical systems. ${ }^{[37,38]}$

\section{Supporting Information}

Supporting Information is available from the Wiley Online Library or from the author.

Acknowledgements

K.Z. thanks the German Research Foundation (DFG) for the financial support of the project ZH546/3-1. The program "Material Science Wood” from Lower Saxony Ministry of Science and Culture, Germany, is gratefully acknowledged for the financial support. X.W. and H.L. thank China Scholarship Council (CSC) for the financial support of their Ph.D. fellowships.

\section{Conflict of Interest}

The authors declare no conflict of interest.

\section{Keywords}

cellulose nanocrystals, co-nonsolvency actuation, hydrogel actuators, thermo-responsiveness, tunable motion

\section{References}

[1] P. Weis, D. Wang, S. Wu, Macromolecules 2016, 49, 6368.

[2] W. Z. Qian Zhao, Yingwu Luo, Tao Xie, Sci. Adv. 2016, 2.

[3] H. Huang, X. Wang, F. Rehfeldt, K. Zhang, ACS Sustain. Chem. Eng. 2018, 6, 4294. 
[4] Y. Tan, D. Wang, H. Xu, Y. Yang, W. An, L. Yu, Z. Xiao, S. Xu, Macromol. Rapid Commun. 2018, 39, e1700863.

[5] H. Huang, Y. Wang, X. Wang, F. Rehfeldt, K. Zhang, Macromol. Rapid Commun. 2017, 38.

[6] S. Son, E. Shin, B. S. Kim, Biomacromolecules 2014, 15, 628.

[7] S. Wang, P. Huang, X. Chen, ACS nano 2016, 10, 2991.

[8] A. Mourran, H. Zhang, R. Vinokur, M. Moller, Adv. Mater. 2017, 29.

[9] H. Yuk, S. Lin, C. Ma, M. Takaffoli, N. X. Fang, X. Zhao, Nat. Commun. 2017, 8, 14230.

[10]H. Banerjee, M. Suhail, H. Ren, Biomimetics 2018, 3, 15.

[11]M. L. Xiong Pu, Xiangyu Chen, Jiangman Sun, Chunhua Du, Yang Zhang, Junyi Zhai, Weiguo Hu, Zhong Lin Wang, Sci. Adv. 2017, 3.

[12]S. Lin, H. Yuk, T. Zhang, G. A. Parada, H. Koo, C. Yu, X. Zhao, Adv. Mater. 2016, 28, 4497.

[13] X. Pu, M. Liu, X. Chen, J. Sun, C. Du, Y. Zhang, J. Zhai, W. Hu, Z. L. Wang, Sci. adv. 2017, 3, e1700015.

[14]Y. S. Kim, M. Liu, Y. Ishida, Y. Ebina, M. Osada, T. Sasaki, T. Hikima, M. Takata, T. Aida, Nat. Mater. 2015, 14, 1002.

[15]E. Wang, M. S. Desai, S. W. Lee, Nano lett. 2013, 13, 2826.

[16]K. Miyamae, M. Nakahata, Y. Takashima, A. Harada, Angew.Chem.Int. Ed. 2015, 54, 8984.

[17]M. Nakahata, Y. Takashima, A. Hashidzume, A. Harada, Angew. Chem. 2013, 52, 5731. [18]D. Morales, E. Palleau, M. D. Dickey, O. D. Velev, Soft matt. 2014, 10, 1337. [19]X. Zhang, C. L. Pint, M. H. Lee, B. E. Schubert, A. Jamshidi, K. Takei, H. Ko, A. Gillies, R. Bardhan, J. J. Urban, M. Wu, R. Fearing, A. Javey, Nano lett. 2011, 11, 3239. [20]D. Kim, H. Kim, E. Lee, K. S. Jin, J. Yoon, Chem. Mater. 2016, 28, 8807.

[21]P. Košovan, T. Richter, C. Holm, Macromolecules 2015, 48, 7698.

[22]L. Ionov, Mater. Today 2014, 17, 494.

[23]L. Ionov, Adv. Funct. Mater. 2013, 23, 4555. 
[24]K. Iwaso, Y. Takashima, A. Harada, Nat. Chem. 2016, 8, 625.

[25]C. Ma, X. Le, X. Tang, J. He, P. Xiao, J. Zheng, H. Xiao, W. Lu, J. Zhang, Y. Huang, T. Chen, Adv. Funct. Mater. 2016, 26, 8670.

[26]W. Kong, C. Wang, C. Jia, Y. Kuang, G. Pastel, C. Chen, G. Chen, S. He, H. Huang, J. Zhang, S. Wang, L. Hu, Adv. Mater. 2018, e1801934.

[27]Y. Shi, C. Ma, L. Peng, G. Yu, Adv. Funct. Mater. 2015, 25, 1219.

[28]J. C. Breger, C. Yoon, R. Xiao, H. R. Kwag, M. O. Wang, J. P. Fisher, T. D. Nguyen, D. H. Gracias, ACS applied materials \& interfaces 2015, 7, 3398.

[29]D. Han, C. Farino, C. Yang, T. Scott, D. Browe, W. Choi, J. W. Freeman, H. Lee, ACS Appl. Mater. Interfaces 2018, 10, 17512.

[30] S. Jiang, F. Liu, A. Lerch, L. Ionov, S. Agarwal, Adv. Mater. 2015, 27, 4865.

[31]T. Chen, H. Bakhshi, L. Liu, J. Ji, S. Agarwal, Adv. Funct. Mater. 2018, 28, 1800514.

[32] S. Xiao, Y. Yang, M. Zhong, H. Chen, Y. Zhang, J. Yang, J. Zheng, ACS Appl. Mater. Interfaces 2017, 9, 20843.

[33]T. Wang, D. Liu, C. Lian, S. Zheng, X. Liu, Z. Tong, Soft matter 2012, 8, 774.

[34] Y. Tan, D. Wang, H. Xu, Y.Yang, W. An, L. Yu, Z. Xiao S. Xu, Macromol. Rapid Commun. 2018, 39, 1700863.

[35]M. J. A. Hore, B. Hammouda, Y. Li, H. Cheng, Macromolecules 2013, 46, 7894.

[36]D. C. E. C. I. Bischofberger, V. Trappe, Soft matter 2014, 10, 8288.

[37] E. Smela, Adv. Mater. 2003, 15, 481.

[38]L. Zhang, P. Naumov, X. Du, Z. Hu, J. Wang, Adv. Mater. 2017, 29. 


\section{Publication 1 (Supporting information)}

\section{Multi-responsive bilayer hydrogel actuators with programmable and precisely tunable motions}

Xiaojie Wang, Heqin Huang, Huan Liu, Florian Rehfeldt, Xiaohui Wang, Kai Zhang*

This file includes:

Experimental section

Figure S1. Representative conductive titration curve of CNCs.

Figure S2. Representative tensile test curves of as-prepared various cooperating PHEAm layers.

Figure S3. Young's modulus of the swollen cooperating PHEAm layers.

Table S1. Nitrogen contents of CNCs- $\mathrm{NH}_{2}$ and CNCs-MAm, and calculated amounts of methyl acrylamide groups.

Movie S1. Grabbing process of the BHAs gripper during the thermal actuation.

Movie S2. Releasing process of the BHAs gripper during the thermal actuation. 


\section{Experimental section.}

\section{Materials}

Microcrystalline cellulose (MCC) with a granule size of $50 \mu \mathrm{m}, 2,2,6,6$-tetramethylpiperidine 1-oxyl (TEMPO), N-hydroxyethyl acrylamide (HEAm), hexamethylenediamine, methacrylic anhydride, N-isopropylacrylamide (NIPAm), N,N'-Methylenebisacrylamide (MBAm), carbonyldiimidazole (CDI), sodium bromide and 2-hydroxy-1-[4-(2-hydroxyethoxy) phenyl]2-methyl-1-propanone (I2959) were purchased from Sigma-Aldrich (USA). Triethylamine was bought from Merck Millipore (USA). Sodium hydroxide $(\mathrm{NaOH})$ was ordered from VWR (Germany). N,N-Bis (acryloyl) cystamine and sodium hypochlorite solution ( $\mathrm{NaClO}$ ) $(11 \% \sim 15 \%)$ were provided by Alfa Aesar (USA). Lithium phenyl-2,4,6trimethylbenzoylphosphinate (LAP) was synthesized according to the report. ${ }^{[1]}$ The organic solution, including N,N-dimethylformamide (DMF), ethanol and acetone were all purchased from TH. Geyer (Germany). DI water utilized throughout all experiments was purified on a Millipore system.

\section{Preparation of cellulose nanocrystals (CNCs) and cellulose nanocrystals with surface- attached methyl acrylamide (CNCs-MAm).}

CNCs were firstly synthesized via the TEMPO-mediated oxidation of MCC according to previous report. ${ }^{[2]}$ Then, CNCs was repeatedly washed with acetone and DMF, before $600 \mathrm{mg}$ CNCs was redispersed in $35 \mathrm{~mL}$ DMF. Carboxyl groups on the CNCs surface were activated by CDI (219 mg, $1.35 \mathrm{mmol})$ for $30 \mathrm{~min}$. Then, hexamethylendiamine $(5.23 \mathrm{~g}, 45 \mathrm{mmol})$ was mixed with CNCs suspension. The amidation reaction was performed at $80^{\circ} \mathrm{C}$ under $\mathrm{N}_{2}$ gas protection for $48 \mathrm{~h}$. The resulted $\mathrm{CNCs}-\mathrm{NH}_{2}$ was then washed with DMF for three times. Afterwards, $300 \mathrm{mg}$ CNCs- $\mathrm{NH}_{2}$ was redispersed in $15 \mathrm{~mL}$ DMF, $60 \mathrm{mg}$ triethylamine was added to the $\mathrm{CNCs}-\mathrm{NH}_{2}$ dispersion and the mixture was degassed by $\mathrm{N}_{2}$ gas for $15 \mathrm{~min}$. After that, methacrylic anhydride $(92 \mathrm{mg}, 0.6 \mathrm{mmol})$ dissolved in $6 \mathrm{~mL}$ DMF was gradually added dropwise to the mixture. The reaction was stirred at room temperature for $24 \mathrm{~h}$. Finally, the 
resulted CNCs-MAm was gradually washed with acetone and DI water. CNCs-MAm was dispersed in DI water and stored in $4^{\circ} \mathrm{C}$ fridge for later use.

\section{Preparation of the cooperating layers of nanocomposite hydrogels.}

Hydrogels of poly(N-hydroxyethylacrylamide) (PHEAm), PHEAm reinforced with neutral CNCs (PHEAm/CNCs) and PHEAm reinforced with crosslinkable CNCs-MAm (PHEAm/CNCs-MAm) were prepared by in situ free radical polymerization in water. Typically, an aqueous suspension of $8 \mathrm{mg}$ CNCs or CNCs-MAm was mixed with the solution of HEAm (92 mg, $0.8 \mathrm{mmol})$ and N,N-Bis (acryloyl) cystamine (1 mg, $0.004 \mathrm{mmol}$ ) to form the gel precursor $(400 \mu \mathrm{l})$. Then, LAP $(2 \mathrm{mg})$ was added to the precursor solution as the photo initiator and this mixture was degassed with $\mathrm{N}_{2}$ gas for 3 min. After that, the solution was injected into a transparent homemade mold. Afterwards, this precursor was exposed to the UV light to perform the polymerization for $30 \mathrm{~min}$.

\section{Fabrication of bilayer hydrogel actuators (BHAs).}

BHAs were composed of the first layer of PNIPAm (active layer) and the second layer of PHEAm with various compositions (cooperating layer). A precursor solution containing NIPAm (0.09 g, $0.8 \mathrm{mmol})$, MBAm (0.6 mg, $\left.4 \times 10^{-3} \mathrm{mmol}\right)$ and $12959(2 \mathrm{mg})$ was degassed by $\mathrm{N}_{2}$ gas for $3 \mathrm{~min}$. The solution was then injected into the mold and UV irradiated for $1 \mathrm{~h}$ to finish the polymerization. After the formation of the first layer, the second layer was synthesized above the existing PNIPAm layer after pouring the precursor solutions into the mould and following polymerization. Three different precursor solutions were used as described above. After the polymerization, the bilayer hydrogels were immersed in DI water to get the fully swollen state. Finally, BHAs were cut into diverse dimensions for further experiments.

Dynamic thermal actuation performance of BHAs. 
For strip-shaped BHAs, BHAs were cut into strips with different aspect ratios (length: $27 \mathrm{~mm}$, width: $9 \mathrm{~mm}, 4.5 \mathrm{~mm}$ and $3 \mathrm{~mm}$ ). Then, the actuation process of BHAs started with immersion of BHAs in DI water of $40{ }^{\circ} \mathrm{C}$ within transparent petri dishes. After complete actuation, BHAs were transferred into DI water of $23{ }^{\circ} \mathrm{C}$. The bending motions were recorded using digital camera from the top of the petri dishes over the whole actuation time.

For cross-shaped BHAs, BHAs were cut into cross-shape and they were connected to a long needle with two pieces of thin PVC sheet. Then, they were used to pick up and transfer a PHEAm hydrogel block (fully swollen, $1 \mathrm{~g}$ ) as target object. The manipulation process was recorded by a digital camera. To tune the maximum weight of hydrogel blocks, various numbers of small nails ( $\sim 0.25 \mathrm{~g}$ each) were pressed into the PHEAm hydrogel blocks.

\section{Dynamic solvent actuation performance of BHAs.}

Various BHA were cut into strips (length: $27 \mathrm{~mm}$, width: $3 \mathrm{~mm}$ ). Then, the specimens were all immersed into pure ethanol in petri dishes to reach the equilibrium state. The bending motion of BHAs was modulated continuously by adding DI water to change the ethanol/water ratio, which was gradually transferred into pure water. The reverse process was conducted by gradually adding ethanol to the pure DI water and eventually being transferred into pure ethanol. The bending motion over the whole time was recorded using a digital camera from the top of the petri dishes.

\section{Characterization and measurements}

Determination of the content of carboxyl groups on CNCs via conductivity titration.

The content of the surface-exposed carboxyl groups of CNCs was determined by electric conductivity titration. $10 \mathrm{ml}$ of aqueous $\mathrm{CNCs}$ suspension where the $\mathrm{pH}$ value was adjusted to 2 with $0.1 \mathrm{M} \mathrm{HCl}$ was titrated with $0.05 \mathrm{M} \mathrm{NaOH}$ using the 800 Dosimat (Metrohm) employing 
a dosing rate of $0.01 \mathrm{ml} / \mathrm{s}$, while the conductivity was recorded using the 856 Conductivity Module (Metrohm) with an interval of $2 \mathrm{~s}$.

Determination of the content of methyl acrylamide groups on CNCs-MAm via elemental analysis.

The content of methyl acrylamide groups was calculated according to the results of elemental analysis (Table S1). The contents of carbon, hydrogen and nitrogen were determined on an Elemental Analyser 4.1 vario EL III (Elementar, Germany).

\section{Scanning electron microscopic measurement of BHAs microstructure}

The microstructures of BHAs samples were observed on a LEO Supra-35 High-Resolution Field Emission Scanning Electron Microscope (Carl Zeiss AG, Germany). To prepare the samples, the BHAs in DI water were frozen in liquid nitrogen to fix the original microstructure. After lyophilization in a freeze drier (Christ Alpha 1-2 LD Plus) at $-28{ }^{\circ} \mathrm{C}$ under a vacuum of $10^{-2}$ mbar, the fracture of the samples was measured.

Size and morphology of CNCs and CNCs-MAm via transmission electron microscopy (TEM)

The shape and size of CNCs-MAm were characterized with TEM. The TEM observation was conducted on a CM 12 Transmission Electron Microscope (Philips, Netherland). The samples were prepared from its aqueous suspension with a concentration of $0.01 \mathrm{wt} \%$. The specimens were stained by using phosphotungstic acid aqueous solution $(2 \mathrm{wt} \%$ in water with the $\mathrm{pH}$ value adjusted to 7.0 using $1 \mathrm{M} \mathrm{NaOH}$ ). 


\section{Mechanical properties tests of the hydrogels.}

The rheological properties of the various PHEAm hydrogels were investigated with a stresscontrolled bulk rheometer (Anton Paar MCR501) by using a $25 \mathrm{~mm}$ parallel plate set-up. In each test, $500 \mu$ hydrogel of each sample was prepared in advance with a thickness of $1 \mathrm{~mm}$. G', G' and tan (delta) were recorded. All the rheology measurements were carried out at room temperature $\left(23^{\circ} \mathrm{C}\right)$.

Tensile tests were performed on a Z3 micro tensile test machine from Grip-Engineering Thümler GmbH. The samples of various PHEAm hydrogels for tensile tests were synthesized in the dumbbell shaped mold (Samples overall length: $46.5 \mathrm{~mm}$; width: $8 \mathrm{~mm}$; inner width: 3.5 $\mathrm{mm}$, gauge length: $10.5 \mathrm{~mm}$, thickness: $3 \mathrm{~mm}$ ) and were immersed in DI water to get their fully swollen state. The swollen PHEAm hydrogels were used for tensile tests and the distance between clamps was set at $\sim 15 \mathrm{~mm}$ during the tests. The BHAs samples were all cut into strip shape (length: $27 \mathrm{~mm}$, width: $3 \pm 0.5 \mathrm{~mm}$, thickness: $2 \pm 0.5 \mathrm{~mm}$ ) and the distance between clamps was set at around $2 \mathrm{~mm}$ during the tests. The default tensile speed was $10 \mathrm{~mm} / \mathrm{min}$. All the tests were performed at $21^{\circ} \mathrm{C}$ and a relative humidity of $65 \%$.

\section{Swelling behaviors of various PHEAm layers in water.}

The swollen hydrogel samples were all completely dried at $60{ }^{\circ} \mathrm{C}$. Then, they were put into DI water and stored at room temperature. The weight changes of the hydrogels were recorded during the swelling to the constant weight at different swelling times after wiping off the excess surface water with paper tissue. The swelling ratio (SR) of the hydrogels at different times was calculated using equation (S1):

$$
\mathrm{SR}=\mathrm{Wt} / \mathrm{Wd} \times 100 \%
$$

where $\mathrm{Wt}$ is the weight of the gel at swelling time $\mathrm{t}, \mathrm{Wd}$ is the weight of the dried hydrogel. 


\section{Swelling behaviors of various PHEAm and PNIPAm in ethanol/water mixtures.}

The swollen PHEAm and PNIPAm hydrogel samples were all immersed into pure ethanol and stored at room temperature for 1 day to reach the equilibrium state. Then, DI water was gradually added to the ethanol to decrease the ethanol content, and the equilibrium weight changes of the hydrogel were recorded during this process at different ethanol content after wiping off the excess surface liquid with paper tissue. The swelling ratio (SR) of the hydrogels at different ethanol content was calculated using equation (S2):

$$
\mathrm{SR}=\mathrm{W} \phi / \mathrm{Ws} \times 100 \%
$$

where $\mathrm{W} \phi$ is the weight of the gel at a certain ethanol content $\phi$, Ws is the weight of the fully swollen hydrogel in water.

\section{Using the Timoshenko equation to illustrate the motion amplitude of thermal actuation.}

This different performance in motion magnitude can be explained using the Timoshenko equation, which can be extended to describe the experimental results qualitatively and semiquantitatively:

$$
k=\frac{6\left(\alpha_{n}-\alpha_{c}\right)(1+x)^{2}}{h\left[3(1+x)^{2}+(1+x y)\left(x^{2}+\frac{1}{x y}\right)\right]}
$$

where $k$ is the curvature, $\mathrm{x}$ is the ratio of layer thicknesses $\mathrm{x}=\mathrm{h}_{\mathrm{c}} / \mathrm{h}_{\mathrm{n}}$, and $\mathrm{y}$ is the ratio of their Young's moduli $\mathrm{y}=\mathrm{E}_{\mathrm{c}} / \mathrm{E}_{\mathrm{n}}$, the subscript $\mathrm{n}$ represents the PNIPAm layer and $\mathrm{c}$ represents the cooperating layers (PHEAm layers). Finally, $\alpha$ represents the free actuation strain of each layer, when it is not combined together. The cooperating layers had negligible response to the experiment temperature, thus $\alpha_{c}=0$. The BHAs with diverse cooperating layers were prepared with the same length and they shared similar thickness ratio $(\mathrm{x} \approx 1)$. Finally, the predominant difference of BHAs is the cooperating layers, which exhibit diverse curvatures defined as $\mathrm{k}_{1}$ $(\mathrm{BHAs} / \mathrm{P}), \mathrm{k}_{2}(\mathrm{BHAs} / \mathrm{CNCs})$ and $\mathrm{k}_{3}(\mathrm{BHAs} / \mathrm{CNCs}-\mathrm{MAm})$. Thus, the equation (S3) can be 
simplified as:

$$
k=\frac{24 \alpha_{n}}{h\left(14+y+\frac{1}{y}\right)}
$$

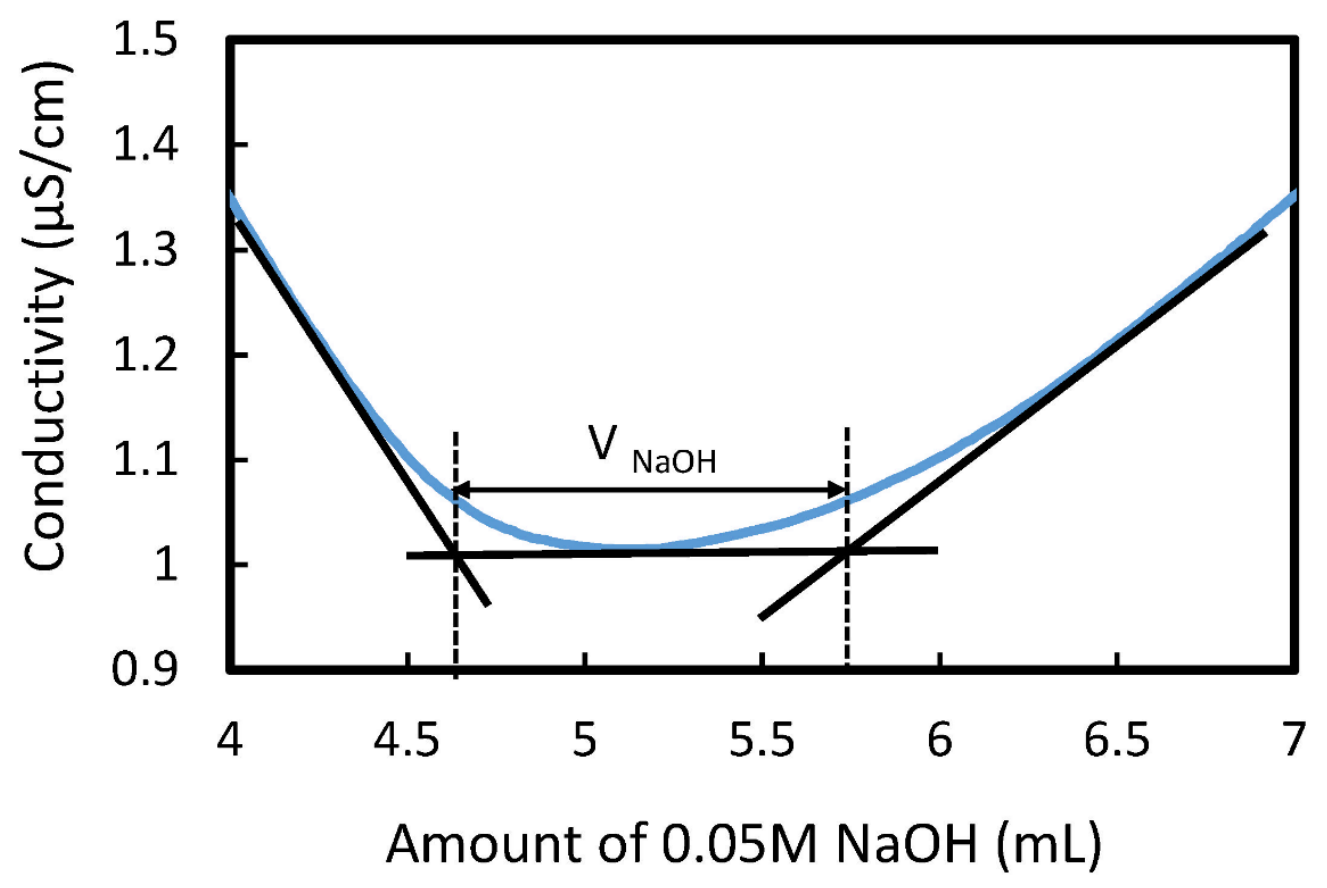

Figure S1. Representative conductive titration curve of CNCs.

The surface of CNCs was decorated with carboxyl groups of $1.37 \pm 0.2 \mathrm{mmol} \mathrm{g}{ }^{-1}$. 


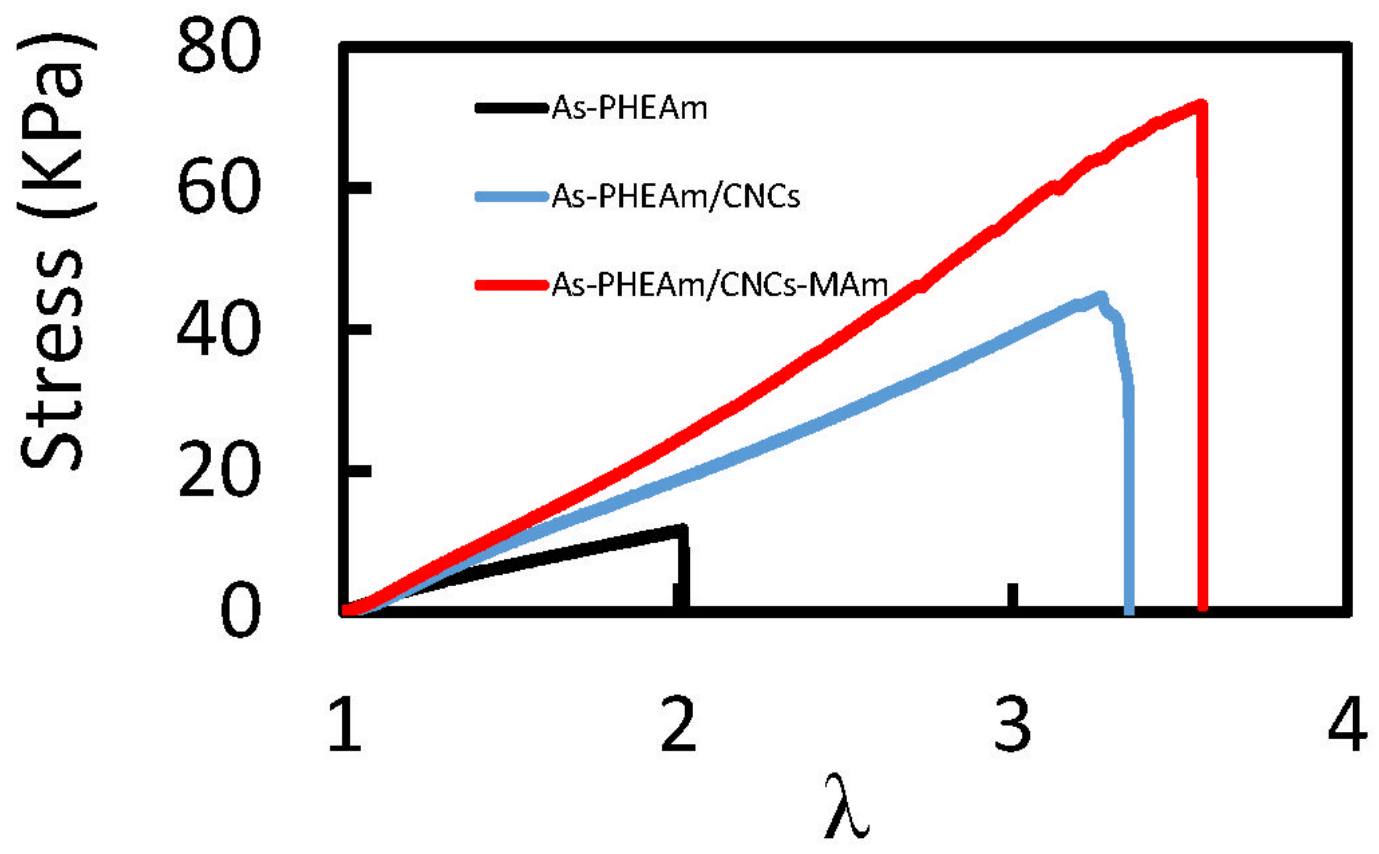

Figure S2. Representative tensile test curves of as-prepared various cooperating PHEAm layers.

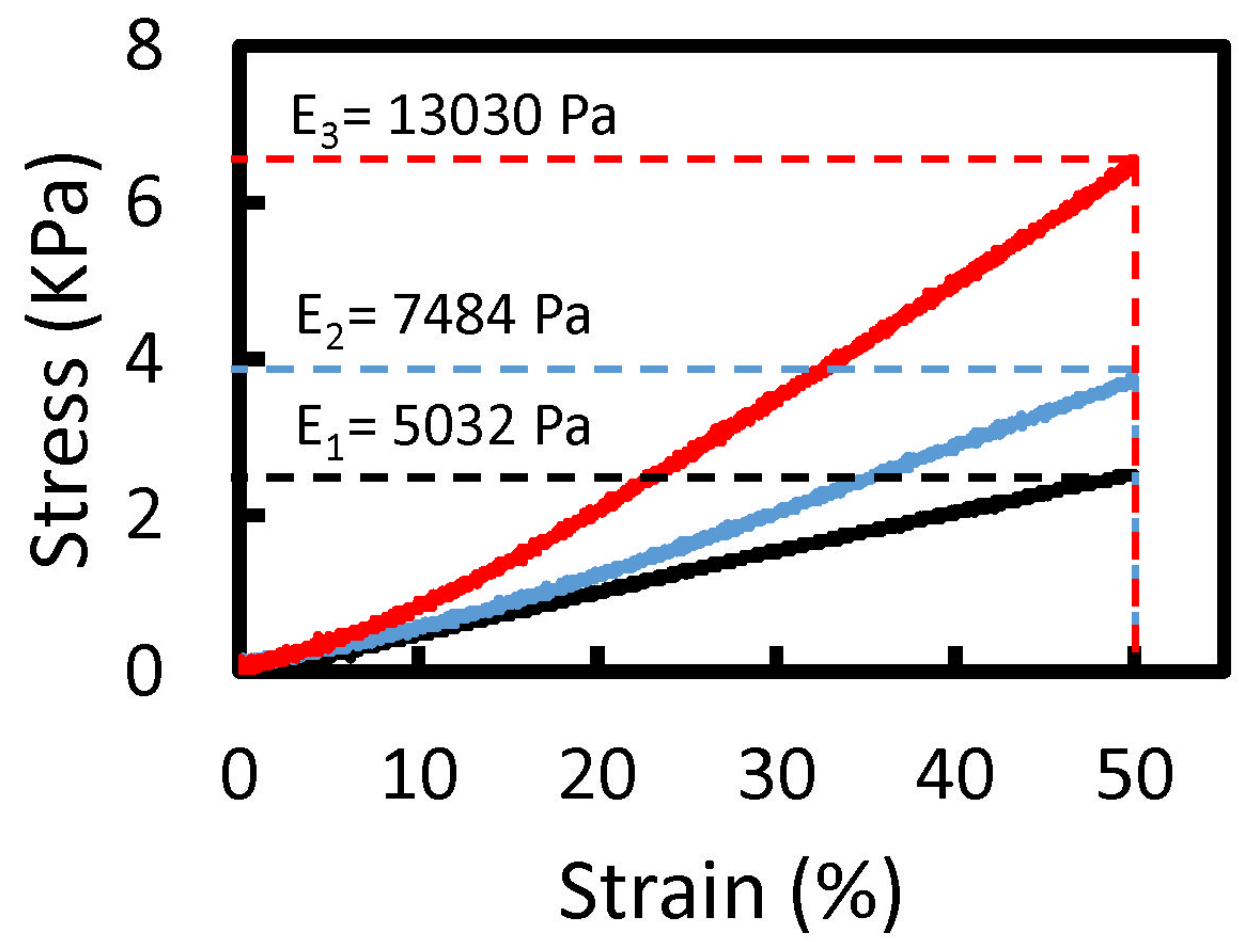

Figure S3. Young's modulus of the swollen cooperating PHEAm layers. E1, E2, E3 represent the Young's modulus of PHEAm, PHEAm/CNCs and PHEAm/CNCs-MAm, respectively. 
Table S1. Nitrogen contents of CNCs- $\mathrm{NH}_{2}$ and CNCs-MAm, and calculated amounts of methyl acrylamide groups.

\begin{tabular}{c|c|c|c}
\hline Samples & $\begin{array}{c}\text { Nitrogen contents in } \\
\text { CNCs-NH2 }(\mathrm{wt} \%)\end{array}$ & $\begin{array}{c}\text { Nitrogen contents in } \\
\text { CNCs-MAm (wt\%) }\end{array}$ & $\begin{array}{c}\text { Amounts of MAm in } \\
\text { CNCs-MAm (mmol/g) }\end{array}$ \\
\hline 1 & 1.97 & 1.9 & 0.54 \\
\hline 2 & 1.65 & 1.59 & 0.55 \\
\hline 3 & 1.8 & 1.74 & 0.51 \\
\hline
\end{tabular}

The amounts of methyl acrylamide groups on CNCs-MAm were determined using the elemental analysis. For this purpose, both $\mathrm{CNCs}^{-\mathrm{NH}_{2}}$ and $\mathrm{CNCs}-\mathrm{MAm}$ samples were measured.

Based on the results of elemental analysis, the content of amine groups on $\mathrm{CNCs}-\mathrm{NH}_{2}$ was calculated as:

$$
\mathrm{N}=14 * 2 \times \lambda
$$

where $\mathrm{N}$ was the weight content of nitrogen in $\mathrm{CNCs}-\mathrm{NH}_{2} ; 14$ is the molecular weight of nitrogen $(\mathrm{g} / \mathrm{mol}) ; \lambda$ is the assumed molar content of amine groups on $\mathrm{CNCs}^{-\mathrm{NH}_{2}}(\mathrm{~mol} / \mathrm{g})$. The molar content of amine groups was calculated to be $0.7 \mathrm{mmol} \mathrm{g}^{-1}$.

The content of methyl acrylamide (MAm) on CNCs-MAm was further calculated based on:

$$
\mathrm{N}^{\prime}=\mathrm{N} /(1+68 \mathrm{x})
$$

where $\mathrm{N}^{\prime}$ is the weight content of nitrogen in CNCs-MAm; $\mathrm{N}$ is the weight content of nitrogen in $\mathrm{CNCs}-\mathrm{NH}_{2} ; 68$ is the increased molecule weight during the transfer from $\mathrm{CNCs}^{-\mathrm{NH}_{2}}$ to CNCs-MAm. $\mathrm{x}$ is the assumed molar content of methyl acrylamide on CNCs-MAm. The molar content of methyl acrylamide was calculated to be $0.5 \pm 0.05 \mathrm{mmol} \mathrm{g}^{-1}$. 


\section{References}

[1] B. D. Fairbanks, M. P. Schwartz, C. N. Bowman, K. S. Anseth, Biomaterials 2009, 30, 6702.

[2] H. Huang, Y. Wang, X. Wang, F. Rehfeldt, K. Zhang, Macromol. Rapid Commun. 2017, 38. 


\section{Publication 2}

\section{Temperature-Responsive, Manipulable Cavitary Hydrogel Containers}

\section{by Macroscopic Spatial Surface-Interior Separation}

Xiaojie Wang, ${ }^{1}$ Yang Yang, ${ }^{1,2}$ Heqin Huang, ${ }^{1}$ and Kai Zhang ${ }^{*, 1}$

${ }^{1}$ Wood Technology and Wood Chemistry, Department of Wood Technology and Woodbased Composites, Georg-August-University of Göttingen, Büsgenweg 4, D-37077

Göttingen, Germany

${ }^{2}$ State Key Laboratory of Pulp and Paper Engineering, South China University of

Technology, Guangzhou, 510640, P. R. China

* Corresponding Author: Kai Zhang

E-mail: kai.zhang@uni-goettingen.de

Manuscript 
KEYWORDS: hydrogel container, surface-interior separation, dynamic hydrogel, crosslinking gradient, temperature-responsive, sustained release

ABSTRACT: Synthetic macroscopic materials transforming from bulk solid or semisolid to closed structure with inner cavities as well as distinct outer and inner microstructures is rarely reported. Here, we report an in situ method for directing spatial surface-interior separation from bulk dynamic hydrogels to closed three-dimensional (3D) hydrogel containers with inner cavities via constructing competitively crosslinking gradient within dynamic hydrogels. The initial crosslinking of phenylboronic acid/catechol complexes is disrupted by stronger ferric ions/catechol associations, generating gradually weakened crosslinking from outside to inside. Both stronger crosslinking in the outer shells and sequentially weaker crosslinked interior generated during swelling closed hydrogel container with tunable dense outer shell, fluffy inner layer and cavities in the core. Cellulose nanocrystals could be used to significantly improve the spatial distinction of gradient crosslinking within hydrogels, leading to even denser outer shell with tunable shell thickness. Moreover, cavitary hydrogel containers with diverse shapes can be programmed by designing the initial shapes of dynamic hydrogels, and macroscopic assembly of individual dynamic hydrogels based on their self-healing capability after subsequent surface-interior separation. These cavitary hydrogel containers demonstrate thermal-responsive gate systems with unique sustained release at higher temperature, and potential reaction containers for oxygen generation on demand. This facile spatial surface- 
interior separation strategy for fabricating closed cavity systems has great potential for various applications.

\section{INTRODUCTION}

In nature, many living organisms of plants and animals, such as gourd, bamboo, swim bladder and blood vessels, rely on their self-generated cavitary structures in the interior to support their essential life activities, for instance mass transportation, self-adaptable movement and mechanical support. ${ }^{1}$ Inspired by these advanced architectures, various synthetic structures with cavities have been fabricated to suit a broad range of applications, for example artificial blood vessels, ${ }^{2}$ hydraulic or pneumatic robotics, ${ }^{3}$ and scaffolds for tissue engineering. ${ }^{4}$

Synthetic materials with cavitary structures were mainly achieved by curing polymer solutions around sacrificial templates, ${ }^{5-7}$ while in situ transformation of synthetic solid materials to structures with inner cavities is still much less reported. Using internal stress changes of stimuli-responsive polymers within materials can generate inhomogeneous deformations, leading to shape transformation to cavitary structures. For example, certain stimuli responsive soft actuators, ${ }^{8-13}$ and shape memory materials, ${ }^{14-19}$ can be triggered to self-roll to open tubes based on uneven stress distribution. Obtained cavitary structures were spatial changes in open and non-continuous 3D shapes and only adaptable to specific environments with the transient characteristics. Some other hydrogels with cavitary structures were fabricated by using catalyst-decomposition method, which fulfilled solid to hollow transformation. ${ }^{20-22}$ However, those transformations need further catalyst system and the approaches for the preparation 
highly demand complicated techniques to fulfill those transformations, ${ }^{23-24}$ which poses great barrier for their applications.

Hydrogels are macroscopically continuous but microscopically open materials with good permeability due to their highly porous structures, which offer the possibility to modify their spatial structures from their macroscopic semi/solid state. ${ }^{25-26}$ Although few hydrogels were successfully transformed into closed cavitary structures, ${ }^{27}$ continuous closed cavitary structures with distinct inner and outer surfaces on the macroscopic scale are not known. In particular, cavitary structures with distinct inner and outer surfaces are often essential for many functions from mass transportation to protection of biologically active species, such as trachea with smooth outer wall and ciliated inner wall. ${ }^{28}$ As well, an efficient method is still lacking for semi/solid materials to evolve in situ into continuously closed cavitary materials with distinct inner and outer walls.

Herein, we present a novel approach to fabricate cavitary hydrogel containers with distinct microstructures in inner and outer walls derived from macroscopic dynamic hydrogels with the same initial compositions by constructing a crosslinking gradient from the surface to the interior with weak dynamic bonds. Furthermore, hydrogel containers with various 3D shapes can be generated by designing the initial shapes of dynamic hydrogels and the macroscopic assembly of individual dynamic hydrogels relying on their excellent self-healing property. Based on the cavitary structure in the interior and thermal responsive poly $(\mathrm{N}$-isopropyl acrylamide) (PNIPAm), these Janus hydrogel containers demonstrated controllable sustained release of water-soluble compounds at higher temperature, yet distinguished from typical 
thermally controlled fast-release system, and the inner cavitary structure can also be used as transferable oxygen generator.

\section{EXPERIMENTAL SECTION}

Materials. Microcrystalline cellulose (MCC), 2,2,6,6-tetramethylpiperidine 1-oxyl (TEMPO), 3-(acrylamido) phenylboronic acid (PBAAm), methacrylic anhydride, phosphotungstic acid hydrate, 2,4,6-trimethylbenzoyl chloride, Iron (III) chloride hexahydrate and Iron(II) chloride tetrahydrate were purchased from Sigma-Aldrich (Germany). Dopamine hydrochloride, sodium hypochlorite solution (11-15\%), hydrogen peroxide (35\%), dimethyl phenylphosphonite and 2-butanone were provided by Alfa Aesar (USA). Sodium sulfate anhydrous, disodium tetraborate were obtained from Merck Millipore (USA). Sodium hydroxide was ordered from VWR (Germany). N-Isopropylacrylamide (NIPAm) was purchased from Acros Organics (Belgium). Sodium bicarbonate was bought from TH Geyer (Germany). Hydrochloric acid (37\%) was obtained from AppliChem (Germany). Organic solvents, including tetrahydrofuran (THF), ethyl acetate, n-hexane and acetone were all obtained from TH Geyer (Germany). Deionized water (DI water) utilized throughout all experiments was purified from a Millipore system. Dopamine methylacrylate ${ }^{29}$ and lithium phenyl-2,4,6-trimethylbenzoylphosphinate (LAP) ${ }^{30}$ were synthesized with previous reported methods.

Preparation of cellulose nanocrystals (CNCs). CNCs were synthesized via the TEMPOmediated oxidation of MCC according to previous report. ${ }^{31}$ After dialysis, obtained CNCs were 
stored at room temperature for $24 \mathrm{~h}$ and precipitated large particles were removed. The upper stable suspension was stored in $4{ }^{\circ} \mathrm{C}$ fridge for later use. The surface of CNCs was decorated with carboxyl groups of $1.34 \pm 0.2 \mathrm{mmol} \mathrm{g}^{-1}$ (Figure S1).

Preparation of dynamic hydrogels. Dynamic hydrogels were prepared via UV initiated radical polymerization. Typically, alkaline aqueous solutions of NIPAm (0.6 mmol), PBAAm $(0.0075 \mathrm{mmol})$ and dopamine methylacrylate $(0.0075 \mathrm{mmol})$ were prepared with borax-NaOH buffer at $\mathrm{pH} 10$ to form the gel precursors. In the presence of CNCs, CNCs $(2 \mathrm{wt} \%$ of whole hydrogel precursor) were mixed with these precursors. Then, LAP $(0.5 \mathrm{wt} \%)$ was added as photoinitiator and the precursors were purged with $\mathrm{N}_{2}$ gas for 3 min to remove dissolved oxygen gas. Afterwards, the precursors were exposed to the UV light for $30 \mathrm{~min}$ to finish the polymerization and gelation process. Flat dynamic hydrogels were formed between two glass slides with thickness of $1 \mathrm{~mm}$, while tubular hydrogels and cylinder hydrogels were generated in their respective molds.

Preparation of hydrogel containers. The dynamic hydrogels of arbitrary dimensions were immersed into ferric chloride solutions $(0.1 \mathrm{M})$ for 1 to $8 \mathrm{~min}$. Afterwards, those samples were immediately rinsed and transferred to DI water to dialyze to constant weights with regularly refreshed water, until hydrogel containers formed with inner cavitary structure. The specimens without $\mathrm{CNCs}$ were named as "HCs/P", the corresponding specimens combined with $\mathrm{CNCs}$ were named as "HCs/CNCs".

Release of Rhodamine B. Rhodamine B releasing experiment was performed in $40 \mathrm{~mL}$ DI water at $25^{\circ} \mathrm{C}$ and $37^{\circ} \mathrm{C}$ by getting out $1 \mathrm{~mL}$ of solution and refilling $1 \mathrm{~mL}$ water with certain 
time intervals. All the measured hydrogel containers were firstly immersed in $20 \mathrm{~mL}$ Rhodamine B solution $(0.1 \mathrm{mg} / \mathrm{mL})$ for $24 \mathrm{~h}$. All the solutions with released Rhodamine B were measured to determine the cumulated amount of released Rhodamine B using the Ultraviolet-visible (UV-Vis) spectroscopy.

Preparation of transferable oxygen generator. The cavitary hydrogel containers $\mathrm{HCs} / \mathrm{CNCs}$ with 3 min treatment in ferric solutions were firstly immersed in $20 \mathrm{~mL}$ mixed solution of $\mathrm{FeCl}_{2} \cdot 4 \mathrm{H}_{2} \mathrm{O}(0.1 \mathrm{M})$ and $\mathrm{FeCl}_{3} \cdot 6 \mathrm{H}_{2} \mathrm{O}(0.2 \mathrm{M})$ for $24 \mathrm{~h}$. Then, the diffused iron ions were allowed to in situ form iron oxide nanoparticles in $\mathrm{NaOH}$ solution (2 M) for $48 \mathrm{~h}$. Thus, the $\mathrm{HCs} / \mathrm{CNCs} 3 \mathrm{~min}$ were incorporated with iron oxide nanoparticles (IONPs), named as HCs/CNCs-IONPs. After that, $\mathrm{HCs} / \mathrm{CNCs}-\mathrm{IONPs}$ specimens were immersed in $\mathrm{H}_{2} \mathrm{O}_{2}$ solution $(10 \mathrm{wt} \%)$ to generate oxygen gas.

Characterizations. Scanning electron microscopy (SEM) was applied to observe the microstructures of freeze-dried samples with LEO Supra-35 High-Resolution Field Emission Scanning Electron Microscope (Carl Zeiss AG, Germany) or an EVO LS15 Scanning Electron Microscope (Carl Zeiss AG, Germany) at an accelerating voltage of $5 \mathrm{kV}$. Swelling ratios for all specimens were obtained from weight change ratios in comparison to initial hydrogels, which are defined as (the weight measured at certain swelling times/weight of initial dynamic hydrogels). The compression and tensile tests were performed with Z3 micro tensile test machine from Grip Engineering Thümler GmbH. The compression tests were performed on the whole hydrogel containers. The tensile tests were measured on dynamic hydrogels and healed dynamic hydrogels with dimensions of $8 \mathrm{~mm} \times 4 \mathrm{~mm} \times 1 \mathrm{~mm}$. To determine the amount 
of Rhodamine B in solutions, Rhodamine B solutions were measured from $400 \mathrm{~nm}$ to $800 \mathrm{~nm}$ using an UV-Vis spectrometer (Analytik Jena, Germany). The temperature-induced volume change was roughly calculated using the water displacement method in grad cylinders by determining the volume change before and after the immersion of hydrogel containers.

\section{RESULTS AND DISCUSSION}

Fabrication of closed Janus hydrogel containers from dynamic hydrogels. A controlled competitive crosslinking method was developed to generate macroscopic hydrogel containers with large inner cavities, as illustrated in Figure 1a. First, initial dynamic hydrogels were prepared comprising of PNIPAm hydrogel crosslinked by the dynamic covalent bonds of phenylboronic acid/catechol complexes in buffer solutions of $\mathrm{pH} 10$ (association constant $\mathrm{K}_{\mathrm{a}}$ $\approx 0.919 \times 10^{3} \mathrm{M}^{-1}$ ) (Figure 1a). ${ }^{17}$ Then, these initial dynamic hydrogels were immersed in ferric chloride buffer solutions ( $0.1 \mathrm{M}$ in borate buffer of $\mathrm{pH} 10)$ for controlled time, which allowed the ferric ions $\left(\mathrm{Fe}^{3+}\right)$ to diffuse into hydrogels. During this process, the $\mathrm{Fe}^{3+}$ ions gradually formed complexes with catechol and replaced initial boronate ester bonds due to their much higher $\mathrm{K}_{\mathrm{a}}\left(10^{37}-10^{40} \mathrm{M}^{-1}\right) .{ }^{18}$ Along with the $\mathrm{Fe}^{3+}$ penetration from outside to inside of hydrogels, a $\mathrm{Fe}^{3+}$ concentration gradient from surface to core formed, resulting in a gradually weaker $\mathrm{Fe}^{3+} /$ catechol crosslinking. After soaking in $\mathrm{Fe}^{3+}$ solutions with certain time before the total complexation of $\mathrm{Fe}^{3+} /$ catechol moieties, hydrogels were transferred into DI water to dialyze until constant weights with regularly refreshed DI water. 


\section{a}

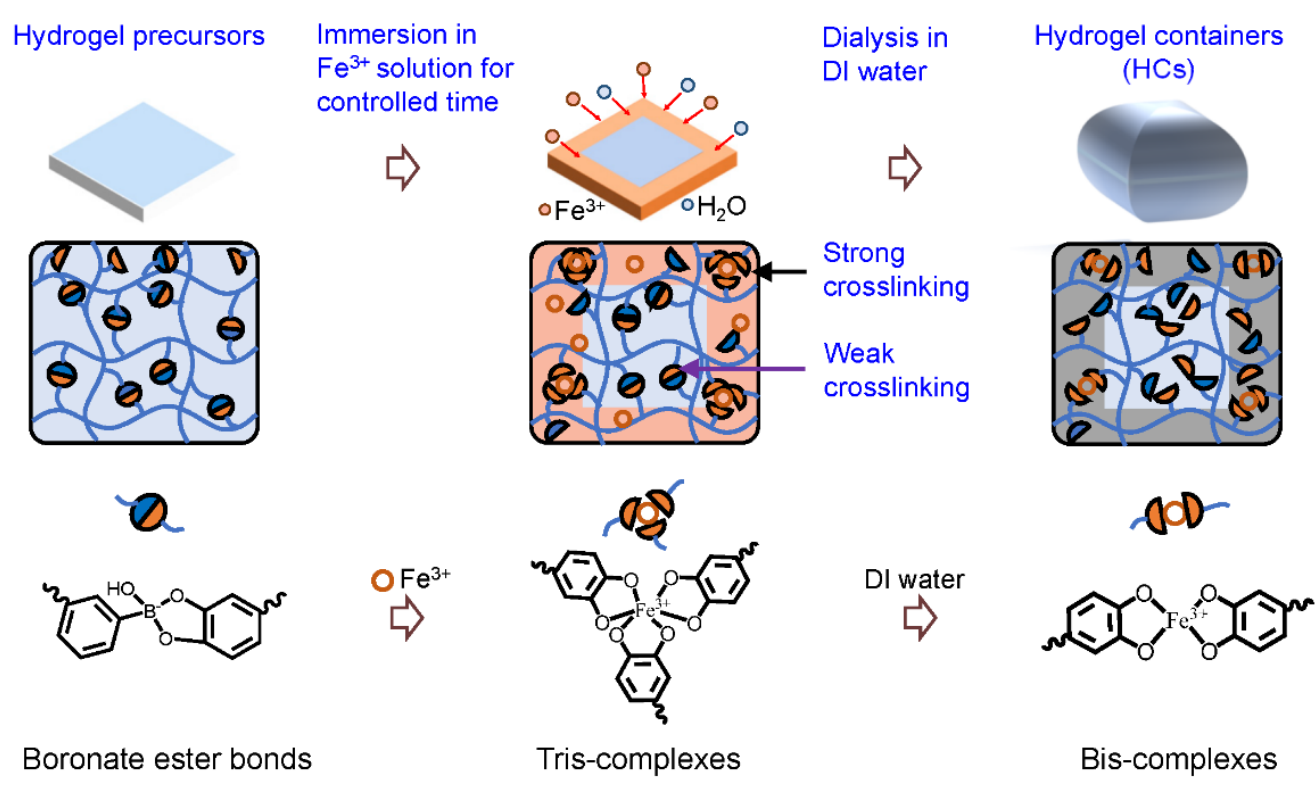

b

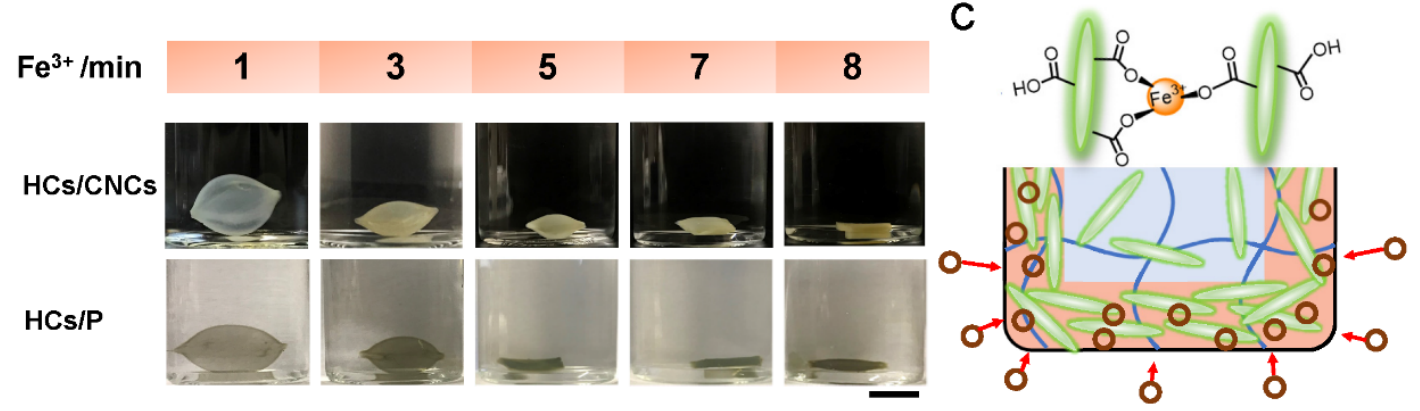

Figure 1. Macroscopic spatial surface-interior separation process from dynamic hydrogels to closed hydrogel containers with inner cavity. (a) Schematic illustration for the preparation of hydrogel containers. PNIPAm dynamic hydrogels crosslinked by boronate ester bonds (phenylboronic acid/catechol complexes) were immersed in ferric chloride solutions for controlled time, in which the catechol moieties within hydrogels form complexes with $\mathrm{Fe}^{3+}$ ions from surface to inside and replace initial boronate ester bonds. During subsequent dialysis in DI water, the hydrogels swell to initiate the surface-interior separation process and the densely crosslinked outer layer of hydrogels forms stable hydrogel shell, while the inner part dominated by boronate ester bonds dissociates into loosely crosslinked polymer chains, leading to cavitary hydrogel containers (HCs). (b) Formation of HCs with various immersion times in 
aqueous $\mathrm{Fe}^{3+}$ solutions. Scale bar: $1 \mathrm{~cm}$. $\mathrm{HCs} / \mathrm{CNCs}$ represented hydrogel containers with CNCs and HCs/P indicated polymeric hydrogel containers without CNCs. (c) Contribution of CNCs during the spatial surface-interior separation process.

During the dialysis, the hydrogels swelled largely that was driven by osmotic pressure, leading to hydrogel containers with encapsulated aqueous solutions. This should be due to the decomposition of weak crosslinking in the interior of hydrogels, while strong crosslinking in the outer layer of hydrogels maintained stable. In parallel, the complexes of $\mathrm{Fe}^{3+} /$ catechol gradually changed from tris-complexes to bis-complexes due to lower $\mathrm{pH}$ in DI water. ${ }^{18}$ It should be noted that the outer layer with strong $\mathrm{Fe}^{3+} /$ catechol crosslinking formed the stable hydrogel shell with negligible oxidation of catechol moieties (Figure S2). At the same time, the inner part where the $\mathrm{Fe}^{3+}$ ions did not reach was still dominated by boronate ester bonds and dissociated into loosely crosslinked polymer chains during swelling. During this swelling process, roughly $35 \mathrm{wt} \%$ of the solid mass was released out of hydrogels through porous hydrogel walls (Figure S3). As the result, the thin flat bulk hydrogels experienced a macroscopic spatial surface-interior separation process and generated continuous closed cavitary hydrogel containers with enclosed solutions (Figure S4).

In addition to polymeric hydrogels, CNCs with exposed carboxyl groups on surface were incorporated into dynamic hydrogels (Figure 1b). It is obvious that CNCs even facilitated the formation of hydrogel containers during the complexation of $\mathrm{Fe}^{3+}$ ions and catechol moieties. CNCs significantly affected the formation process of cavitary hydrogel containers with respect 
to immersion times in aqueous $\mathrm{Fe}^{3+}$ solutions. For the dynamic hydrogels with the initial thickness of $1 \mathrm{~mm}$, maximal 8 min were sufficient to fully crosslink whole hydrogel network and no hydrogel container with cavities was obtained. Stable hydrogel containers $\mathrm{HCs} / \mathrm{CNCs}$ with cavities in the interior were formed after the treatment in $\mathrm{Fe}^{3+}$ solutions for 1 to $7 \mathrm{~min}$. In comparison, without the addition of $\mathrm{CNCs}$, polymeric hydrogel containers $(\mathrm{HCs} / \mathrm{P})$ with similar thickness for initial dynamic hydrogels only appeared with 1-3 min treatment. Longer immersion time already resulted in bulk fully crosslinked solid hydrogels. This evidence suggested that the addition of CNCs enhanced the spatial distinction during the complexation of $\mathrm{Fe}^{3+}$ ions and catechol moieties within hydrogels. Along diffusion route of $\mathrm{Fe}^{3+}$ ions, the carboxyl groups on CNCs surface should also have complexed with $\mathrm{Fe}^{3+}$ ions to provide additional crosslinking compared with polymeric hydrogels. This extra crosslinking apparently retarded the penetration of $\mathrm{Fe}^{3+}$ ions into the inner part of hydrogels and enhanced the spatial crosslinking, leading to extended surface-interior separation for the formation of hydrogel container (Figure 1c). This retardation offered time window to further regulate the formation process for hydrogel containers. Furthermore, this enhanced spatial surface-interior separation by CNCs apparently also accelerated the subsequent swelling process, resulting in shorter equilibration times. As shown in Figure S5, HCs/CNCs with 3 min treatment in $\mathrm{Fe}^{3+}$ solutions were able to reach the equilibrium after about 9 days, whereas $\mathrm{HCs} / \mathrm{P}$ with 3 min treatment in $\mathrm{Fe}^{3+}$ solutions needed roughly 20 days to equilibrium state. Based on expanded time range for the formation of hydrogel containers, cavitary hydrogel containers used in the following refer to those with CNCs. 
a

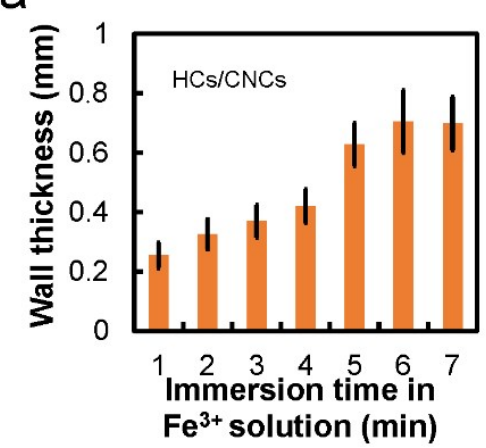

C

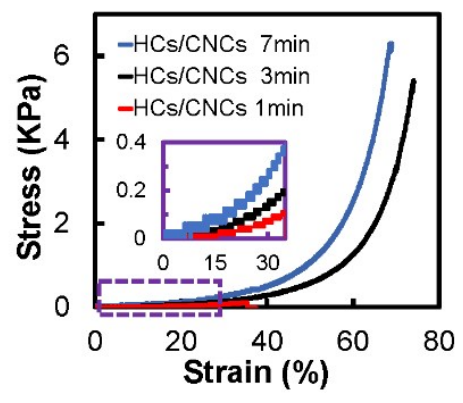

d

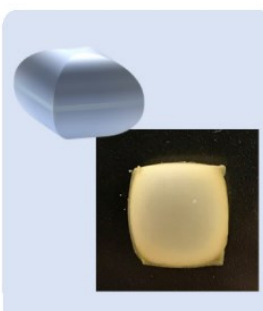

$25^{\circ} \mathrm{C}$ b

$\mathrm{Fe}^{3+} / \min \quad 1$

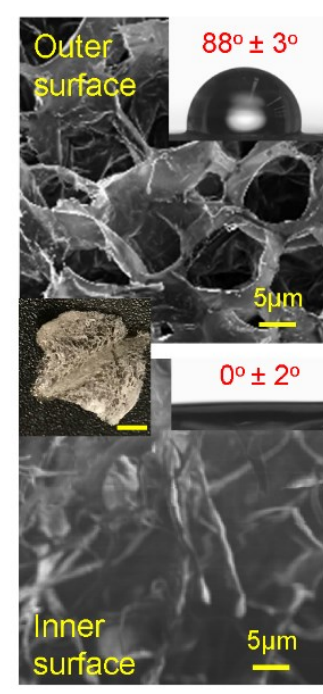

e
3
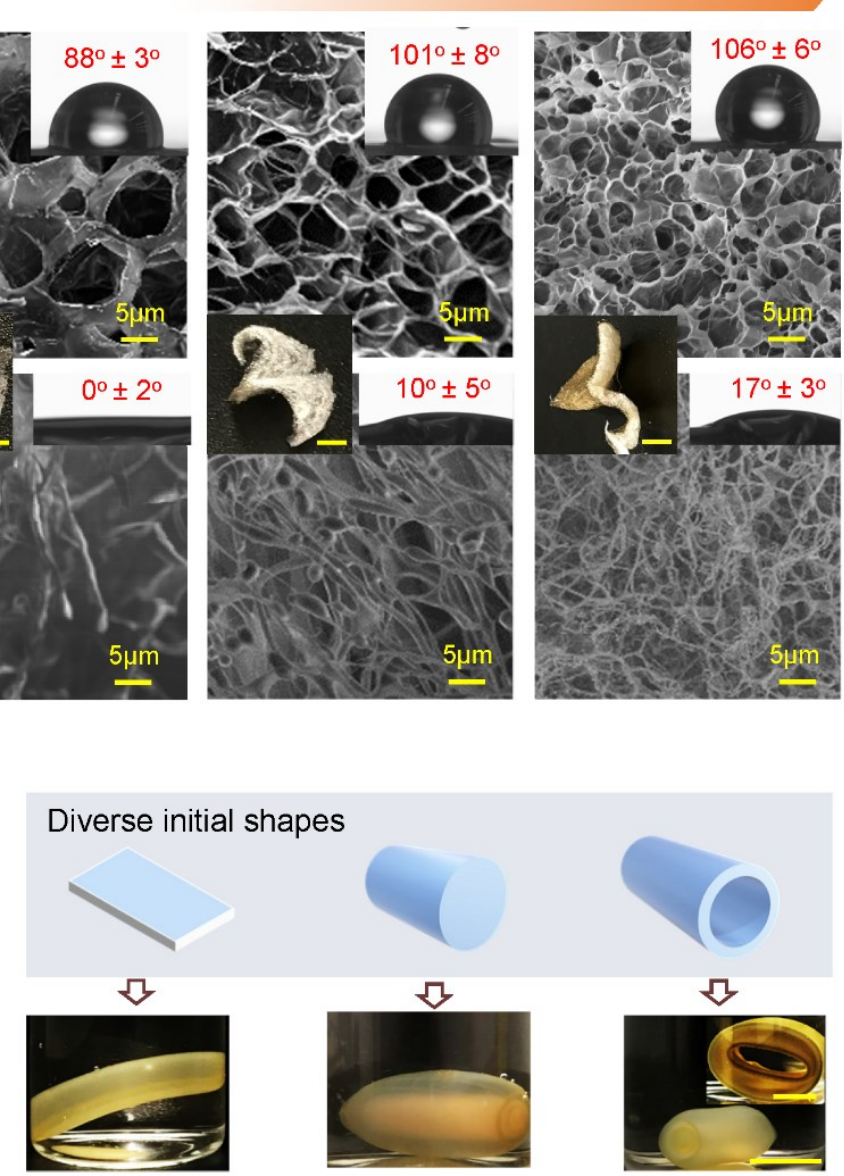

Figure 2. Regulation of microstructures and macrostructure in various HCs/CNCs. (a) Shell thickness of $\mathrm{HCs} / \mathrm{CNCs}$ with increasing immersion times in aqueous $\mathrm{Fe}^{3+}$ solutions. (b) SEM images showing microstructures and surface wettability of outer and inner surface of shells of freeze-dried $\mathrm{HCs} / \mathrm{CNCs}$ with $\mathrm{Fe}^{3+}$-treatment for 1, 3 and $7 \mathrm{~min}$. Insets are the photos of split freeze-dried $\mathrm{HCs} / \mathrm{CNCs}$ with the scale bars of $2 \mathrm{~cm}$ and static water contact angles. (c) Compression tests of $\mathrm{HCs} / \mathrm{CNCs}$ with various treatment times in aqueous $\mathrm{Fe}^{3+}$ solutions. (d) Temperature-responsive $\mathrm{HCs} / \mathrm{CNCs}$ shown with $\mathrm{HCs} / \mathrm{CNCs}$ after 3 min $\mathrm{Fe}^{3+}$-treatment. (e) $\mathrm{HCs} / \mathrm{CNCs}$ with various shapes generated from initial dynamic hydrogels with different initial shapes. Scale bars: $1 \mathrm{~cm}$. 
Morphology and structure control of hydrogel containers. Different soaking times in $\mathrm{Fe}^{3+}$ solutions allowed adjustment of the wall thicknesses, their outer and inner surface morphologies as well as the microstructures in hydrogel containers. Together with increasing immersion time in $\mathrm{Fe}^{3+}$ solutions, the shell thickness of freeze-dried cavitary hydrogel containers increased from roughly $0.22 \mathrm{~mm}$ to $0.75 \mathrm{~mm}$ (Figure 2a). Accompanying with crosslinking change by various soaking times in $\mathrm{Fe}^{3+}$ solutions, as displayed in their scanning electron microscope (SEM) images, these freeze-dried cavitary hydrogel containers after diverse immersion times in $\mathrm{Fe}^{3+}$ solutions had distinct outer and inner layers (Figure $2 \mathrm{~b}$ ). While the outer layers contained porous microstructures as typically crosslinked networks in hydrogels, the inner layer contained a fluffy mat of loosely crosslinked polymer chains. This is because of different crosslinking densities in the outer and internal layers, and the crosslinking became weaker from the outside to the inside, as shown in the cross-sectional SEM image (Figure S6). Moreover, the pore sizes of outer surfaces in hydrogel containers largely decreased from $\sim 9.7 \pm 0.4 \mu \mathrm{m}$ to $\sim 3 \pm 0.5 \mu \mathrm{m}$ with increasing exposure time in $\mathrm{Fe}^{3+}$ solutions from 1 to $7 \mathrm{~min}$, indicating continuous formation of denser layers with extended crosslinking process. At the same time, the inner polymer mat also became denser with longer treatment time in $\mathrm{Fe}^{3+}$ solutions with accompanying higher amount of penetrated $\mathrm{Fe}^{3+}$ ions.

Furthermore, the outer and inner surface of the shells in dried HCs/CNCs displayed different wettabilities, which partly depended on immersion times in $\mathrm{Fe}^{3+}$ solutions. The outer surfaces of hydrogel container became more hydrophobic with the static water contact angle increasing 
from around $88^{\circ}$ to around $106^{\circ}$ because of higher crosslinking densities with extended exposure time in $\mathrm{Fe}^{3+}$ solutions from $1 \mathrm{~min}$ to $7 \mathrm{~min}$. In comparison, the inner surface was highly hydrophilic due to the fluffy polymer mats at room temperature (Figure 2b).

In accordance with more crosslinking after longer treatment in $\mathrm{Fe}^{3+}$ solutions, $\mathrm{HCs} / \mathrm{CNCs}$ with thicker shells became stronger, as shown by their compression tests (Figure 2c). HCs/CNCs with longer treatment in $\mathrm{Fe}^{3+}$ solutions ( 3 and $7 \mathrm{~min}$ ) showing larger deformations behaved similar as stiff hydrogels due to smaller internal volumes. In comparison, $\mathrm{HCs} / \mathrm{CNCs}$ with immersion in $\mathrm{Fe}^{3+}$ solution for 1 min containing a larger internal volume was softer and too brittle to bear compression. The breaking stress of $\mathrm{HCs} / \mathrm{CNCs}$ with 7 min $\mathrm{Fe}^{3+}$-treatment was about 54 times higher than that of $\mathrm{HCs} / \mathrm{CNCs}$ with only 1 min $\mathrm{Fe}^{3+}$-treatment.

The sufficiently strong hydrogel shells after 3 min treatment in aqueous $\mathrm{Fe}^{3+}$ solutions in fact assure stable hydrogel cavity containing encapsulated solutions with varied shapes. Based on the temperature-responsive property of PNIPAm, hydrogel containers showed thermal responsive behaviors and induced alterable shapes. At surrounding temperatures of higher than the lower critical solution temperature (LCST) of PNIPAm, e.g. at $37{ }^{\circ} \mathrm{C}$, the hydrogel containers shrank slightly. Due to the nearly uncompressible aqueous solutions in the interior of hydrogel containers, the cavitary hydrogel containers only underwent limited volume shrinkage. For instance, hydrogel containers after 3 min treatment in aqueous $\mathrm{Fe}^{3+}$ solutions only shrank with a shrinkage ratio of around 0.3 , which is much lower than general bulk PNIPAm-based hydrogels (Figure S7). As a result, the hydrogel containers with an initial pillow shape approached a spherical shape, which allowed the smallest surface area to maintain 
the similar volume (Figure 2d). As well, this shape change is totally reversible based on the reversible, thermal-responsive properties of PNIPAm. Thus, use of the thermal-responsive properties of PNIPAm demonstrates one efficient strategy to tune the shapes of resulting hydrogel containers.

Furthermore, hydrogel containers with diverse shapes were obtained by preparing initial dynamic hydrogels with different pre-designed shapes. As shown in Figure 2e, stable cavitary hydrogel containers in cuboid, cylindrical and tubular shape with closed shells were generated after the complexation with $\mathrm{Fe}^{3+}$ ions and dialysis in DI water.

\section{Macroscopic assembly and shape repair of closed cavitary hydrogel containers. In}

addition, the initial dynamic hydrogels can also be arbitrarily combined for programming more complex shapes based on their self-healing property owing to the dynamic boronate ester bonds. As shown in Figure 3a, two cut-off blocks of flat hydrogel strips can merge together based on the reassociation of boronate ester bonds. The tensile curves for original and healed strips exhibited similar mechanical performance, revealing effective healing behaviors (Figure S8). In particular, the self-healed dynamic hydrogels were still transformed into closed hydrogel containers with inner cavity after subsequent surface-interior separation via the complexation with $\mathrm{Fe}^{3+}$ ions and dialysis. Therefore, individual dynamic hydrogels can be regarded as building blocks for programming cavitary hydrogel containers with more complex geometries. Following this, arbitrary shape-programming is allowed, which can avoid the dependence on complicated molds for initial dynamic hydrogels. For example, the flat hydrogel strip was 
twisted in 3D Mobius shape and the two ends were merged together by the self-healing properties of dynamic hydrogels. After the surface-interior separation via the complexation with $\mathrm{Fe}^{3+}$ ions and dialysis, a closed cavitary hydrogel container in 3D Mobius band shape was obtained. As well, three flat dynamic hydrogel strips merged together to give a cross-shaped dynamic hydrogel, which was further transformed into cavitary hydrogel container after $\mathrm{Fe}^{3+}$ treatment and dialysis. Therefore, this macroscopic assembly provides a new pathway for designing and programming multi-shaped cavitary hydrogel containers, which is of particular interest for diverse potential applications. 
a Self-healing behavior enabled macroscopic assembly of dynamic hydrogels

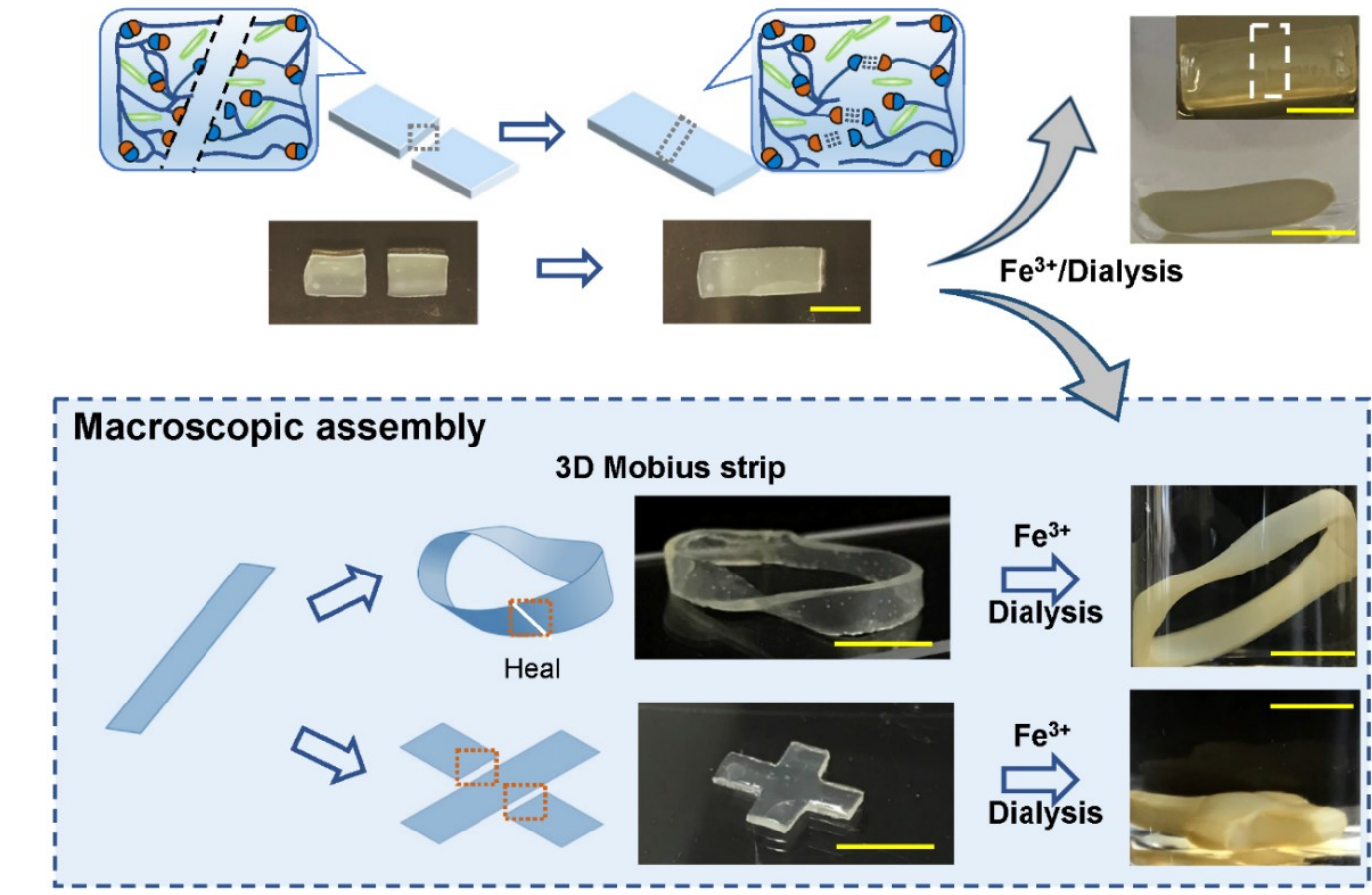

b

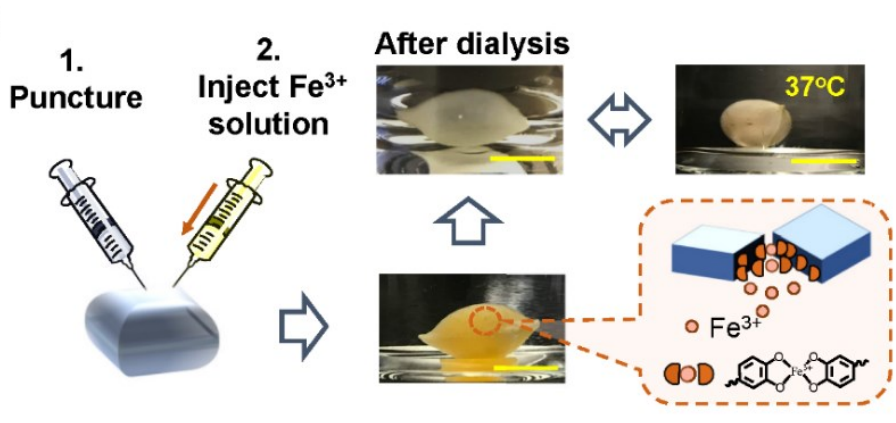

C

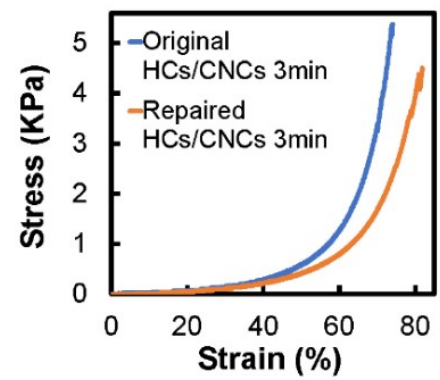

Figure 3. Strategies for shaping and repairing hydrogel containers. (a) Self-healing behavior enabled macroscopic assembly of dynamic hydrogels for shape programming of hydrogel containers. Scale bars: $1 \mathrm{~cm}$. (b) $\mathrm{Fe}^{3+}$-assisted repairable property of hydrogel containers as $\mathrm{HCs} / \mathrm{CNCs}$ with 3 min treatment in aqueous $\mathrm{Fe}^{3+}$ solutions. (c) Compression test strain-stress curves of original and repaired $\mathrm{HCs} / \mathrm{CNCs}$ with 3 min treatment in aqueous $\mathrm{Fe}^{3+}$ solutions. 
In contrast to conventional liquid-filled cavitary structures that are generally very intolerant to punctures, hydrogel containers reported herein with puncture wounds could be easily repaired with the assistance of $\mathrm{Fe}^{3+}$ ions (Figure 3b). The hydrogel containers can be punctured by a syringe, in order to extract the aqueous solution from the cavity of hydrogel containers. After the removal of the solution and the syringe, broken $\mathrm{HCs} / \mathrm{CNCs}$ were caused with the syringe puncture. By injecting $\mathrm{Fe}^{3+}$ solution $(0.1 \mathrm{M})$ into $\mathrm{HCs} / \mathrm{CNCs}$ and subsequent thorough dialysis in water, the puncture caused by syringe was healed, which was implemented by the reassociation of $\mathrm{Fe}^{3+} /$ catechol and $\mathrm{Fe}^{3+} / \mathrm{CNCs}$ complexes by injected $\mathrm{Fe}^{3+}$ ions (Figure $3 \mathrm{~b}$ ). Repaired HCs/CNCs were able to withstand the compression force caused by the shrinkage of PNIPAm shell at $37{ }^{\circ} \mathrm{C}$ and maintained thermal-reversible shape changes, which also confirmed the effective repairing effect. Moreover, the compression tests on original and repaired $\mathrm{HCs} / \mathrm{CNCs}$ demonstrated the efficient recovery of the mechanical properties (Figure 3c). Compared to original hydrogel containers, repaired ones demonstrated slightly lower breaking stress while higher breaking strain. 
a
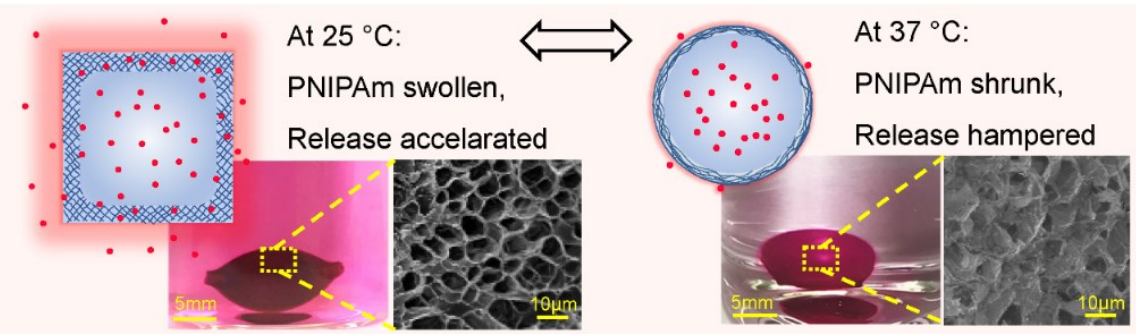

b

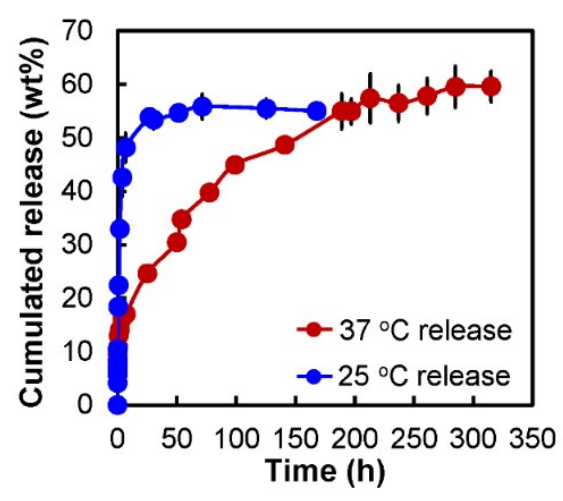

C

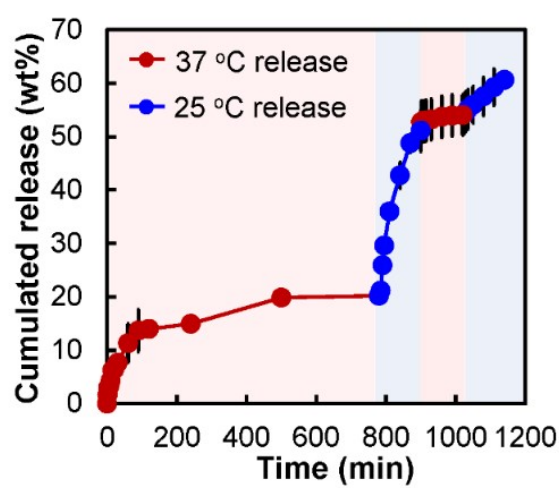

d
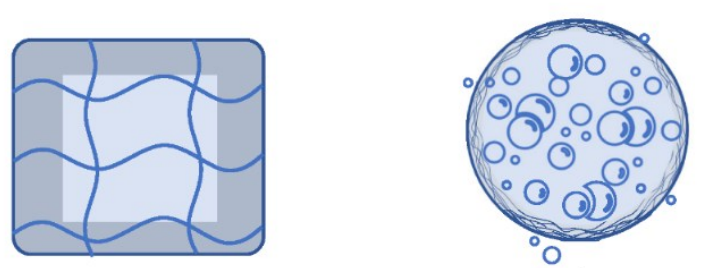

e

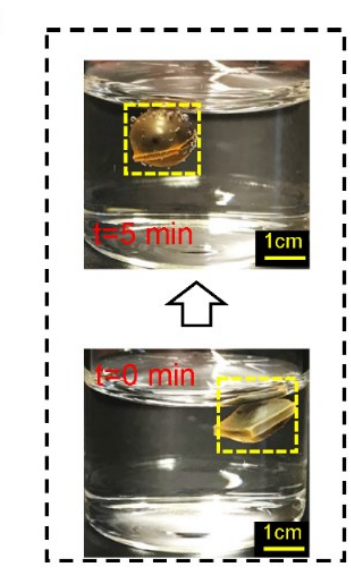

Figure 4. Temperature-responsive release of water-soluble Rhodamine B from cavitary hydrogel containers $\mathrm{HCs} / \mathrm{CNCs}$ after $3 \mathrm{~min} \mathrm{Fe}^{3+}$-treatment and their potential use as reaction containers. (a) Photos and schematic illustration for the release of Rhodamine B from $\mathrm{HCs} / \mathrm{CNCs}$ at $25^{\circ} \mathrm{C}$ and $37^{\circ} \mathrm{C}$. SEM images show the porous structures in the dried hydrogel shells. (b) The cumulated release of Rhodamine B at $25{ }^{\circ} \mathrm{C}$ and $37{ }^{\circ} \mathrm{C}$. (c) Temperatureresponsive release of Rhodamine $B$ with successive temperature switches. d) Schematic illustration for the fabrication of transferrable reaction containers using the cavitary hydrogel containers. e) Photos of $\mathrm{HCs} / \mathrm{CNCs}-\mathrm{IONPs}$ in $\mathrm{H}_{2} \mathrm{O}_{2}$ solution for 0 and $5 \mathrm{~min}$. 


\section{Applications as temperature-controlled gate systems and transferrable reaction}

containers. Based on the large interior volumes and volume phase transition of PNIPAm in polymer networks, cavitary hydrogel containers were then further used for the delivery of low molecular compounds. Different from common PNIPAm-based hydrogels as substrates, PNIPAm networks in our hydrogel container shells represent tunable gates by providing a different delivery behavior. As demonstrated in Figure 4a, the $\mathrm{HCs} / \mathrm{CNCs}$ loading with watersoluble Rhodamine B underwent a burst release at $25{ }^{\circ} \mathrm{C}$ below LCST (around $32{ }^{\circ} \mathrm{C}$ ) of PNIPAm with open porous structures. In contrast, a sustained release was observed at $37{ }^{\circ} \mathrm{C}$ above LCST of PNIPAm, where the pores in hydrogel containers were "closed". Distinguished from conventional burst release of encapsulated compounds from bulk PNIPAm hydrogels at high temperatures due to the squeezing by shrunk PNIPAm, ${ }^{32}$ the release of encapsulated compounds from the interior of hydrogel containers was hindered by the closed pores in the shells at higher temperature. High temperatures resulted in denser and hydrophobic PNIPAm shells with shrunk pores (Figure 4a), but little change in the overall volume (Figure 2d, S7), because the shrinkage of the whole hydrogel containers was restricted by the large volume of aqueous core. As the result, the release of Rhodamine B was significantly retarded, and the encapsulated compounds were retained within the interior of hydrogel containers. In comparison, at lower temperature than LCST, the shell of hydrogel containers recovers to initial state and the pores become larger, allowing the fast release of compounds from the interior of hydrogel containers.

Based on the total reversibility of the dense hydrophobic shell at $37{ }^{\circ} \mathrm{C}$ and loose hydrophilic shell at $25^{\circ} \mathrm{C}$, this PNIPAm-based cavitary hydrogel container can be used as a gate system 
for controlled release. As shown in Figure $4 \mathrm{~b}$, the $\mathrm{HCs} / \mathrm{CNCs}$ underwent a matrix release mode at $25{ }^{\circ} \mathrm{C}$, which was mainly driven by osmotic pressure. The release equilibrium was reached after roughly $24 \mathrm{~h}$. In comparison, a sustained release mode occurred at $37^{\circ} \mathrm{C}$ with the largely retarded equilibrium to around $200 \mathrm{~h}$, further revealing reduced pore sizes at $37^{\circ} \mathrm{C}$. This is significantly different from typical PNIPAm-based bulk materials as temperature-controlled release systems, where the large volume shrinkage quickly squeezes out the water and dissolved agents at the temperatures above $\operatorname{LCST}^{32}$

More importantly, these release systems based on cavitary hydrogel containers can be well controlled by varying surrounding temperatures on demand (Figure 4c). For instance, after the diffusion-out of the Rhodamine B from the hydrogel container shells (as observed for the first $120 \mathrm{~min}$ ) and reaching the temperature of set $37{ }^{\circ} \mathrm{C}$ in the aqueous system, only $6.2 \mathrm{wt} \%$ Rhodamine B could be released in the following 660 min due to the largely reduced hydrogel pore size within the shell of hydrogel containers at $37^{\circ} \mathrm{C}$ and therefore retarded release. Upon lowering the surrounding temperature to $25{ }^{\circ} \mathrm{C}$, a burst release suddenly appeared with the opening pores in the hydrogel shell, and around $31 \mathrm{wt} \%$ of Rhodamine B was released in following $120 \mathrm{~min}$. Then, the release rate could be switched again by raising the temperature to $37{ }^{\circ} \mathrm{C}$ for a slower release and by decreasing to $25{ }^{\circ} \mathrm{C}$ for a fast release. Such switchable release control between sustained/accelerated release can thus be repeatedly achieved, which provides novel temperature-controlled switchable releasing and dosing gate systems.

In addition, the cavitary structure of hydrogel containers also envisioned potential usage as reaction container. As schematically illustrated in Figure 4d, hydrogel container $\mathrm{HCs} / \mathrm{CNCs}$ 
after 3 min $\mathrm{Fe}^{3+}$-treatment was firstly immersed in $\mathrm{Fe}^{2+} / \mathrm{Fe}^{3+}$ mixed solution for $24 \mathrm{~h}$. Then, the diffused iron ions were allowed to in situ form IONPs in $\mathrm{NaOH}$ solution for $48 \mathrm{~h} .{ }^{33}$ The $\mathrm{HCs} / \mathrm{CNCs}$ were thus incorporated with IONPs (HCs/CNCs-IONPs), which can be used to catalyze decomposition of $\mathrm{H}_{2} \mathrm{O}_{2}$ to generate oxygen gas. As shown in Figure 4e, the $\mathrm{HCs} / \mathrm{CNCs}-\mathrm{IONPs}$ were immersed in $\mathrm{H}_{2} \mathrm{O}_{2}$ solution $(10 \mathrm{wt} \%$ ), and the shrunk $\mathrm{HCs} / \mathrm{CNCs}-$ IONPs gradually expanded due to the generation of oxygen gas. This demonstrates promising potential of these cavitary hydrogel containers as transferrable reaction systems.

\section{CONCLUSIONS}

In summary, we demonstrated an in situ approach for macroscopic surface-interior separation from solid dynamic hydrogels to closed cavitary hydrogel containers by constructing competitively crosslinking gradients within hydrogels. The addition of CNCs significantly improved the spatial formation of crosslinking gradient within hydrogel containers, which allowed to tune the unique fluffy internal structures and the shell thickness. The shapes of closed hydrogel containers can be programmed by either designing initial dynamic hydrogels or macroscopic assembly of individual dynamic hydrogels. Furthermore, obtained hydrogel containers were easily repairable via injecting $\mathrm{Fe}^{3+}$ solution, owing to the strong crosslinking kinetics of $\mathrm{Fe}^{3+} /$ catechol complexation. Based on the thermal responsive PNIPAm in shells, yet different from conventional thermal controlled release systems, cavitary hydrogel containers demonstrated a temperature-responsive gate system for sustained release at higher temperatures (above LCST) and accelerated release at lower temperatures. In addition, the 
large cavitary structure in hydrogel containers also demonstrated an oxygen generator, which envisioned great potential as chemical reaction container. This facile solid shaping methodology for fabricating closed cavitary systems has great potential for diverse applications, including controlled drug release, cell culture, chemical reactor and soft actuators.

\section{ASSOCIATED CONTENT}

\section{Supporting Information.}

The Supporting Information is available free of charge on the ACS Publications website.

Supporting figures S1-S6.

Conductive titration curve of CNCs; UV-vis spectra of dynamic hydrogels and the shell of hydrogel containers $\mathrm{HCs} / \mathrm{CNCs}$ after 3 min treatment in $\mathrm{Fe}^{3+}$ solutions; solid weight loss during formation process; Visual appearance of macroscopic spatial surface-interior separation process; Swelling ratios of $\mathrm{HCs} / \mathrm{CNCs}$ and $\mathrm{HCs} / \mathrm{P}$; SEM image of the wall of freeze-dried hydrogel container after 3 min treatment in $\mathrm{Fe}^{3+}$ solutions in cross section; Volume shrinkage ratio for hydrogel container $\mathrm{HCs} / \mathrm{CNCs}$ and bulk hydrogel; Tensile tests of original and selfhealed dynamic hydrogels.

\section{AUTHOR INFORMATION}

\section{Corresponding Author}


*Kai Zhang - Wood Technology and Wood Chemistry, Department of Wood Technology and Wood-based Composites, Georg-August-University of Göttingen, Büsgenweg 4, D-37077 Göttingen, Germany

E-mail: kai.zhang@uni-goettingen.de

\section{Funding Sources}

K.Z. thanks the Federal Ministry for Economic Affairs and Energy (BMWi) and the Ministry for Science and Culture of Lower Saxony (MWK) for the financial support of WIPANO project (FKZ03THW05K14). K.Z. thanks Lower Saxony Ministry of Science and Culture, Germany for the financial support of the program "Material Science Wood".

\section{Notes}

The authors declare no competing financial interest.

\section{ACKNOWLEDGMENT}

K.Z. thanks the Federal Ministry for Economic Affairs and Energy (BMWi) and the Ministry for Science and Culture of Lower Saxony (MWK) for the financial support of WIPANO project (FKZ03THW05K14). K.Z. thanks Lower Saxony Ministry of Science and Culture, Germany for the financial support of the program "Material Science Wood". X.J.W. thanks China scholarship council (CSC) for financial support. Y.Y. thanks the China postdoctoral Science Foundation (2019M652905). We thank Ms. Jiaxiu Wang, Mr. Hua Zhang and Mr. Yawen Yao for the SEM measurements. We thank Mr. Dan Xu for his support during the revision. 


\section{REFERENCES}

(1) Wu, B.; Jian, Y.; Le, X.; Lin, H.; Wei, S.; Lu, W.; Zhang, J.; Zhang, A.; Huang, C. F.; Chen, T., Supramolecular Fabrication of Complex 3D Hollow Polymeric Hydrogels with Shape and Function Diversity. ACS Appl. Mater. Interfaces 2019, 11 (51), 48564-48573.

(2) Cheng, S.; Jin, Y.; Wang, N.; Cao, F.; Zhang, W.; Bai, W.; Zheng, W.; Jiang, X., Selfadjusting, Polymeric Multilayered Roll that can Keep the Shapes of the Blood Vessel Scaffolds During Biodegradation. Adv. Mater. 2017, 29 (28), 1700171.

(3) Yuk, H.; Lin, S.; Ma, C.; Takaffoli, M.; Fang, N. X.; Zhao, X., Hydraulic Hydrogel Actuators and Robots Optically and Sonically Camouflaged in Water. Nat. Commun. 2017, 8 (1), 14230 .

(4) Bouhout, S.; Chabaud, S.; Bolduc, S., Collagen Hollow Structure for Bladder Tissue Engineering. Mater. Sci. Eng. C 2019, 102, 228-237.

(5) Ghanizadeh Tabriz, A.; Mills, C. G.; Mullins, J. J.; Davies, J. A., \& Shu, W., Rapid Fabrication of Cell-Laden Alginate Hydrogel 3D Structures by Micro Dip-Coating. Front. Bioeng. Biotechnol. 2017, 5, 13.

(6) Ma, S.; Rong, M.; Lin, P.; Bao, M.; Xie, J.; Wang, X.; Huck, W. T. S.; Zhou, F.; Liu, W., Fabrication of 3D Tubular Hydrogel Materials through On-Site Surface Free Radical Polymerization. Chem. Mater. 2018, 30 (19), 6756-6768.

(7) Zarket, B. C., Raghavan, S. R., Onion-like Multilayered Polymer Capsules Synthesized by a Bioinspired Inside-out Technique. Nat. Commun. 2017, 8 (1), 1-10. 
(8) Wu, Z. L.; Moshe, M.; Greener, J.; Therien-Aubin, H.; Nie, Z.; Sharon, E.; Kumacheva, E., Three-dimensional Shape Transformations of Hydrogel Sheets Induced by Small-Scale Modulation of Internal Stresses. Nat. Commun. 2013, 4, 1-7.

(9) Fan, W.; Shan, C.; Guo, H.; Sang, J.; Wang, R.; Zheng, R.; Sui, K.; Nie, Z., Dual-Gradient enabled Ultrafast Biomimetic Snapping of Hydrogel Materials. Sci. Adv. 2019, 5 (4), 7174.

(10)Guo, H.; Zhang, Q.; Liu, W.; Nie, Z., Light-Mediated Shape Transformation of a SelfRolling Nanocomposite Hydrogel Tube. ACS Appl. Mater. Interfaces 2020, 12 (11), 1352113528.

(11) Jia, W.; Qiu, X.; Zhang, L., Photocrosslinking Patterning of Single-Layered Polymer Actuators for Controllable Motility and Automatic Devices. ACS Appl. Mater. Interfaces 2019, $11(17), 16252-16259$.

(12) Wei, S.; Lu, W.; Le, X.; Ma, C.; Lin, H.; Wu, B.; Zhang, J.; Theato, P.; Chen, T., Bioinspired Synergistic Fluorescence-Color-Switchable Polymeric Hydrogel Actuators. Angew. Chem. Int. Ed. 2019, 58 (45), 16243-16251.

(13) Le, X.; Lu, W.; Zhang, J.; Chen, T., Recent Progress in Biomimetic Anisotropic Hydrogel Actuators. Adv. Sci. 2019, 6, 1801584.

(14) Peng, L.; Zhu, J.; Agarwal, S., Self-Rolled Porous Hollow Tubes Made up of Biodegradable Polymers. Macromol. Rapid Commun. 2017, 38 (10), 1700034.

(15)Jeon, S. J.; Hauser, A. W.; Hayward, R. C., Shape-Morphing Materials from StimuliResponsive Hydrogel Hybrids. Acc. Chem. Res. 2017, 50 (2), 161-169.

(16)Kim, Y.; Yuk, H.; Zhao, R.; Chester, S. A.; Zhao, X., Printing Ferromagnetic Domains for Untethered Fast-Transforming Soft Materials. Nature 2018, 558 (7709), 274-279. 
(17)Montero de Espinosa, L.; Meesorn, W.; Moatsou, D.; Weder, C., Bioinspired Polymer Systems with Stimuli-Responsive Mechanical Properties. Chem. Rev. 2017, 117 (20), 1285112892.

(18) Ma, C.; Lu, W.; Yang, X.; He, J.; Le, X.; Wang, L.; Zhang, J.; Serpe, M. J.; Huang, Y.; Chen, T., Bioinspired Anisotropic Hydrogel Actuators with On-Off Switchable and ColorTunable Fluorescence Behaviors. Adv. Funct. Mater. 2018, 28 (7), 1704568.

(19) Zhao, Q.; Yang, X.; Ma, C.; Chen, D.; Bai, H.; Li, T.; Yang, W.; Xie, T., A Bioinspired Reversible Snapping Hydrogel Assembly. Mater. Horiz. 2016, 3 (5), 422-428.

(20) Liang, S.; Tu, Y.; Chen, Q.; Jia, W.; Wang, W.; Zhang, L., Microscopic Hollow Hydrogel Springs, Necklaces and Ladders: A Tubular Robot as a Potential Vascular Scavenger. Mater. Horiz. 2019, 6 (10), 2135-2142.

(21) Tu, Y.; Chen, Q.; Liang, S.; Zhao,Q.; Zhou, X.; Huang, W.; Huang, X.; Zhang, L., Antifreezing Heat-Resistant Hollow Hydrogel Tubes. ACS Appl. Mater. Interfaces 2019, 11 (20), 18746-18754.

(22) Chen, Q.; Tan, H.; Tu, Y.; Zhang, L., Experimental Insight into the Evolutionary Mechanism of Solid-to-Hollow Hydrogel. Chem. Commun. 2019, 55 (76), 11470-11473.

(23)Tang, J.; Tong, Z.; Xia, Y.; Liu, M.; Lv, Z.; Gao, Y.; Lu, T.; Xie, S.; Pei, Y.; Fang, D.; Wang, T. J., Super Tough Magnetic Hydrogels for Remotely Triggered Shape Morphing. $J$. Mater. Chem. B 2018, 6 (18), 2713-2722.

(24)Lendlein, A.; Jiang, H.; Jünger, O.; Langer, R., Light-Induced Shape-Memory Polymers. Nature 2005, 434 (7035), 879-882. 
(25)Huang, H.; Wang, X.; Yu, J.; Chen, Y.; Ji, H.; Zhang, Y.; Rehfeldt, F.; Wang, Y.; Zhang, K., Liquid-Behaviors-Assisted Fabrication of Multidimensional Birefringent Materials from Dynamic Hybrid Hydrogels. ACS Nano 2019, 13 (4), 3867-3874.

(26)Hou, S.; Ma, P. X., Stimuli-Responsive Supramolecular Hydrogels with High Extensibility and Fast Self-Healing via Precoordinated Mussel-Inspired Chemistry. Chem. Mater. 2015, 27 (22), 7627-7635.

(27) Han, L.; Zheng, Y.; Luo, H.; Feng, J.; Engstler, R.; Xue, L.; Jing, G,; Deng, X.; Campo, A.; Cui, J., Macroscopic Self-Evolution of Dynamic Hydrogels to Create Hollow Interiors. Angew. Chem. Int. Ed. 2020, 59 (14), 5611-5615.

(28) Boazak, E. M.; Auguste, D. T., Trachea Mechanics for Tissue Engineering Design. ACS Biomater. Sci. Eng. 2018, 4 (4), 1272-1284.

(29)Lee, H.; Lee, B. P.; Messersmith, P. B., A Reversible Wet/Dry Adhesive Inspired by Mussels and Geckos. Nature 2007, 448 (7151), 338-341.

(30)Fairbanks, B. D.; Schwartz, M. P.; Bowman, C. N.; Anseth, K. S., Photoinitiated Polymerization of PEG-Diacrylate with Lithium Phenyl-2,4,6-trimethylbenzoylphosphinate: Polymerization Rate and Cytocompatibility. Biomaterials 2009, 30 (35), 6702-6707.

(31)Huang, H.; Wang, Y.; Wang, X.; Rehfeldt, F.; Zhang, K., Robust Heterogeneous Hydrogels with Dynamic Nanocrystal-Polymer Interface. Macromol. Rapid Commun. 2017, $38(12), 1600810$.

(32)Zhu, Y.; Zeng, Q.; Zhang, Q.; Li, K.; Shi, X.; Liang, F.; Han, D., Temperature/Nearinfrared Light-Responsive Conductive Hydrogels for Controlled Drug Release and Real-Time Monitoring. Nanoscale 2020, 12 (16), 8679-8686. 
(33)Singh, N.; Riyajuddin, S.; Ghosh, K.; Mehta, S. K.; Dan, A., Chitosan-Graphene Oxide Hydrogels with Embedded Magnetic Iron Oxide Nanoparticles for Dye Removal. ACS Appl. Nano Mater. 2019, 2 (11), 7379-7392. 


\title{
Publication 2 (Supporting information)
}

\section{Temperature-Responsive, Manipulable Cavitary Hydrogel Containers by Macroscopic Spatial Surface-Interior Separation}

Xiaojie Wang, ${ }^{1}$ Yang Yang, ${ }^{1,2}$ Heqin Huang, ${ }^{1}$ and Kai Zhang*,I

\author{
${ }^{1}$ Wood Technology and Wood Chemistry, Department of Wood Technology and Wood- \\ based Composites, Georg-August-University of Göttingen, Büsgenweg 4, D-37077 \\ Göttingen, Germany \\ ${ }^{2}$ State Key Laboratory of Pulp and Paper Engineering, South China University of \\ Technology, Guangzhou, 510640, P. R. China
}

* Corresponding Author: Kai Zhang

E-mail: kai.zhang@uni-goettingen.de 


\section{Supporting Figures S1-S8.}

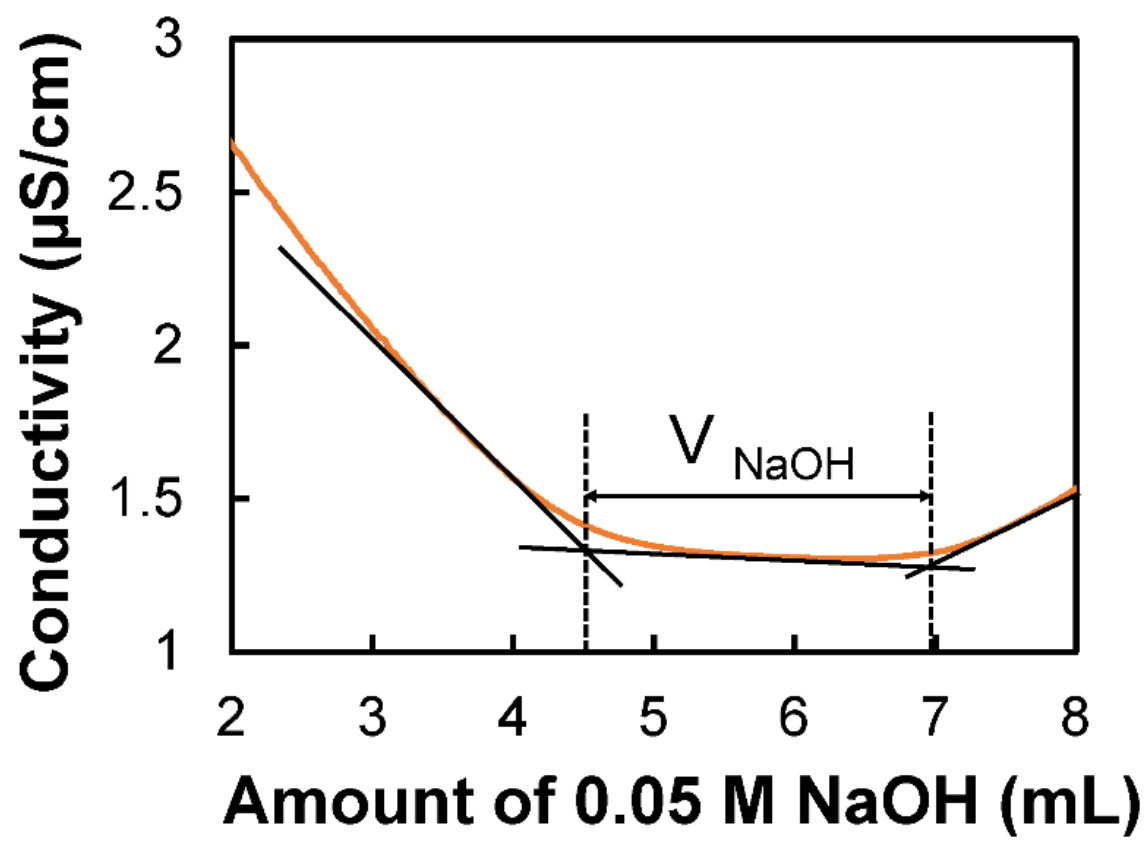

Figure S1. Representative conductivity titration curve of CNCs. $25 \mathrm{~mL}$ of aqueous CNCs suspension $(3.6 \mathrm{mg} / \mathrm{mL})$ was titrated with $0.05 \mathrm{M} \mathrm{NaOH}$ solution using the 800 Dosimat (Metrohm) employing a dosing rate of $0.01 \mathrm{ml} / \mathrm{s}$, while the conductivity was recorded using the 856 Conductivity Module (Metrohm) with an interval of $2 \mathrm{~s}$.

According to the method reported before, ${ }^{1}$ the surface of CNCs was decorated with carboxyl groups of $1.34 \pm 0.2 \mathrm{mmol} \mathrm{g}^{-1}$. 


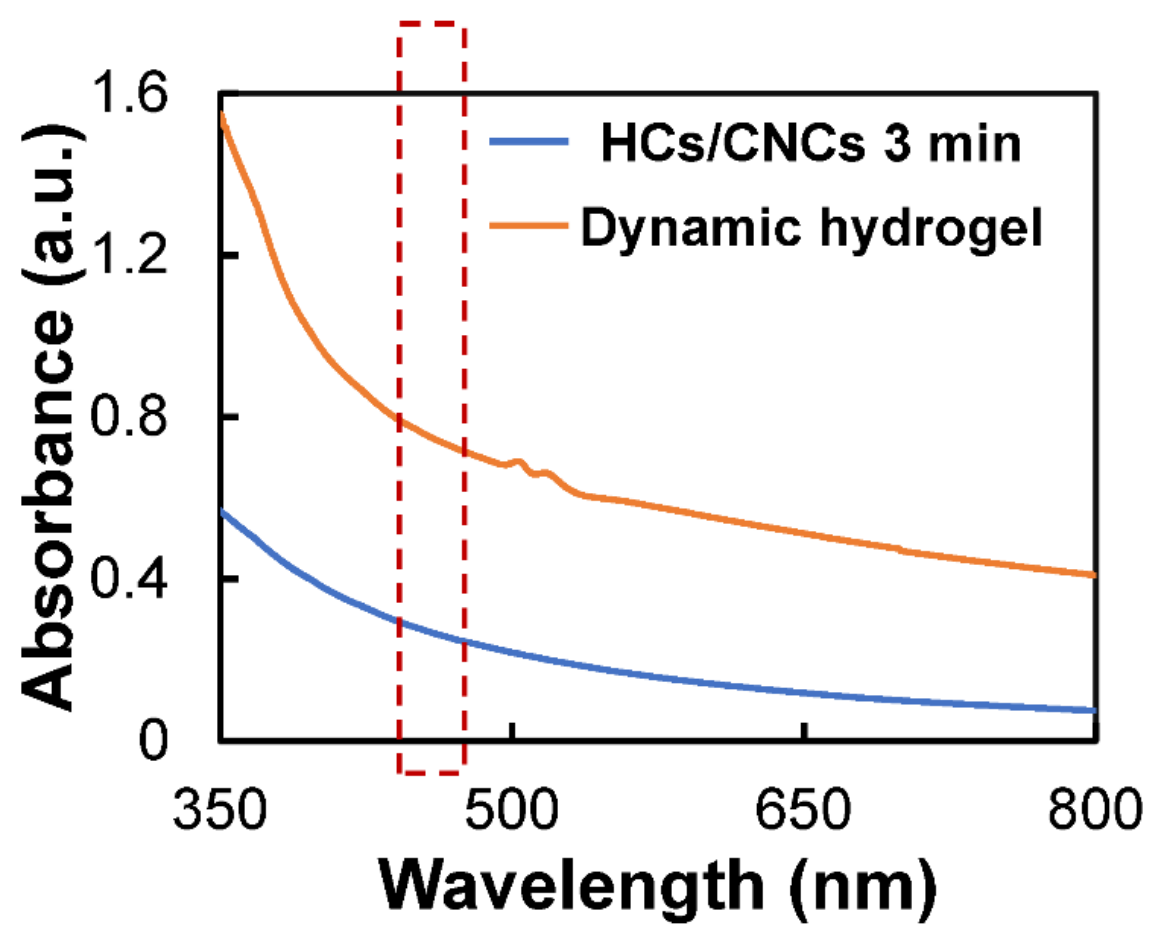

Figure S2. UV-Vis spectra of dynamic hydrogel and the shell of hydrogel containers $\mathrm{HCs} / \mathrm{CNCs}$ after 3 min treatment in $\mathrm{Fe}^{3+}$ solutions.

There was no representative absorbance peak of dicatechol compounds at around $484 \mathrm{~nm}$, indicating negligible oxidation of catechol moieties. 


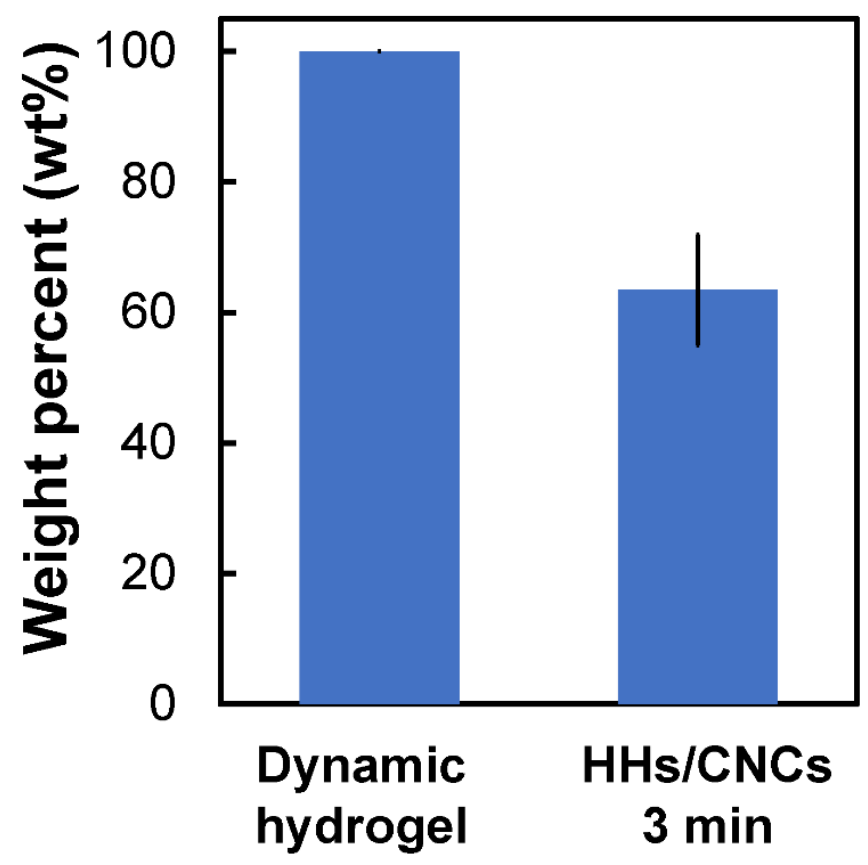

Figure S3. Solid contents of dried hydrogel container $\mathrm{HCs} / \mathrm{CNCs}$ after 3 min treatment in $\mathrm{Fe}^{3+}$ solutions.

Comparing the dried weight of hydrogel containers $\mathrm{HCs} / \mathrm{CNCs}$ with their initial dynamic hydrogels, we can find that roughly $35 \mathrm{wt} \%$ of solid was released from the porous structure of hydrogel containers of $\mathrm{HCs} / \mathrm{CNCs}$ after 3 min treatment in $\mathrm{Fe}^{3+}$ solutions. 


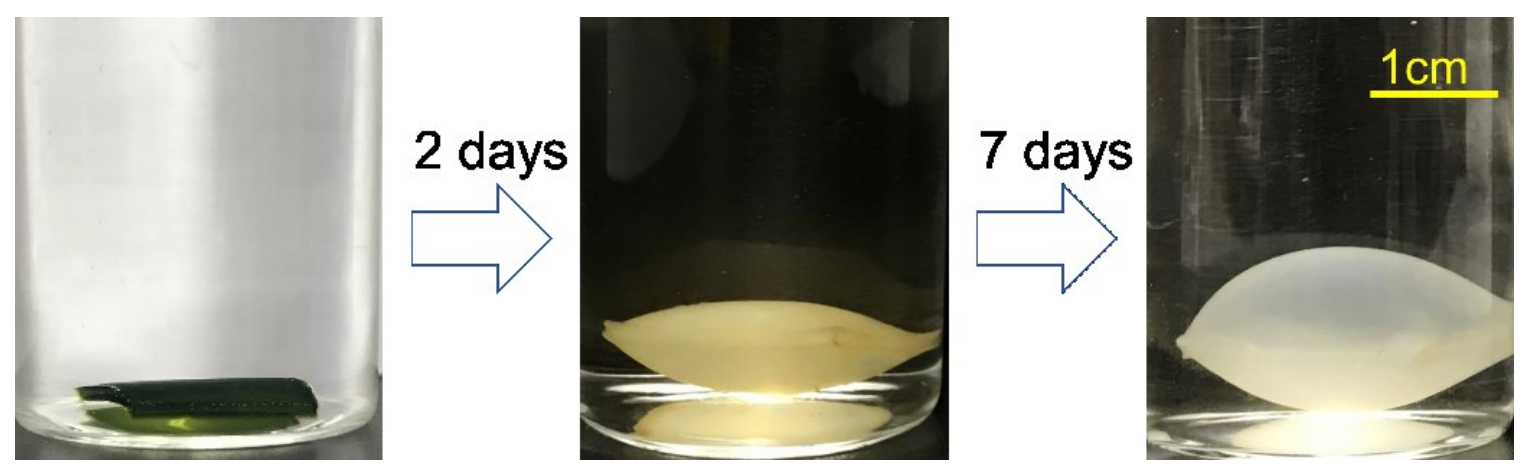

Figure S4. Visual appearance of macroscopic spatial surface-interior separation process from flat bulk hydrogel to 3D closed hydrogel containers of $\mathrm{HCs} / \mathrm{CNCs}$ after 3 min treatment in $\mathrm{Fe}^{3+}$ solutions.

It can be easily seen the flat hydrogel gradually expanded with longer dialysis process, more and more water diffused into the hydrogel to initiate the macroscopic spatial surface-interior separation process. As a result, we obtained the hydrogel container with large inner cavity. 


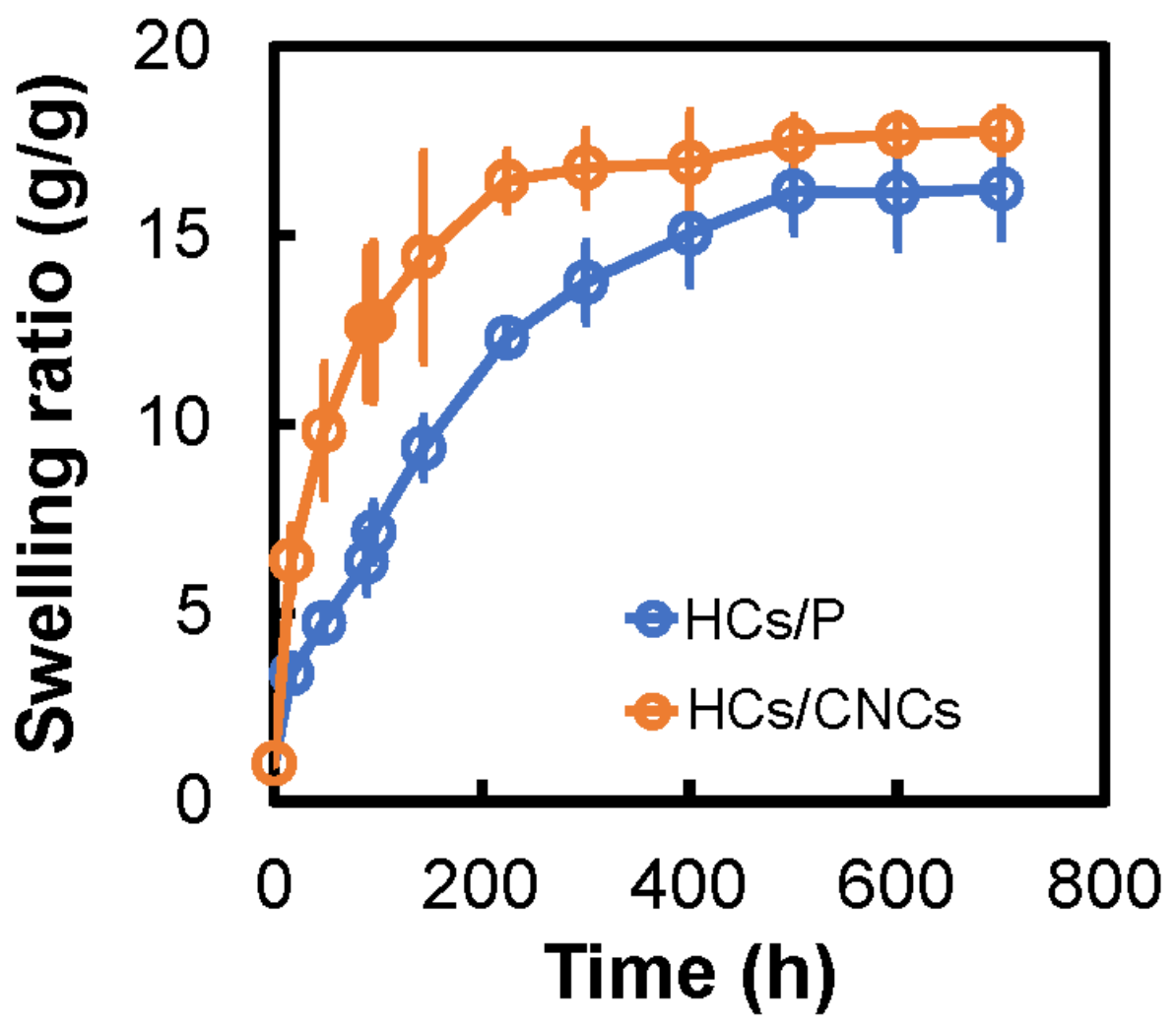

Figure S5. Swelling ratios of $\mathrm{HCs} / \mathrm{CNCs}$ (representing hydrogel containers with CNCs) and $\mathrm{HCs} / \mathrm{P}$ (polymeric hydrogel containers without CNCs).

The addition of CNCs affected the formation process, and $\mathrm{HCs} / \mathrm{CNCs}$ with 3 min treatment in $\mathrm{Fe}^{3+}$ solutions were able to reach the equilibrium after about 9 days, whereas $\mathrm{HCs} / \mathrm{P}$ with 3 min treatment in $\mathrm{Fe}^{3+}$ solutions needed roughly 20 days to equilibrium state. 


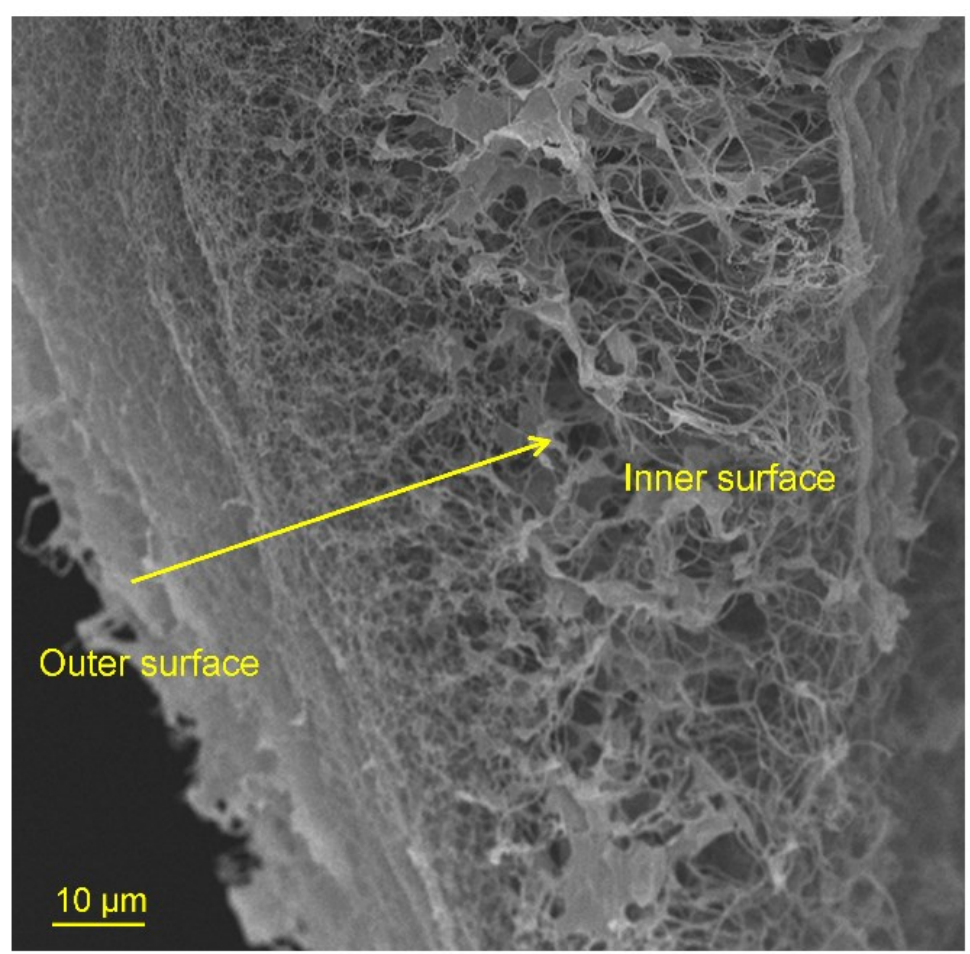

Figure S6. Representative SEM image of the wall of freeze-dried hydrogel container after 3 min treatment in $\mathrm{Fe}^{3+}$ solutions in cross section.

From the outer surface to the interior, the crosslinking became weaker, which further indicates the gradient crosslinking density within the wall of hydrogel container. 


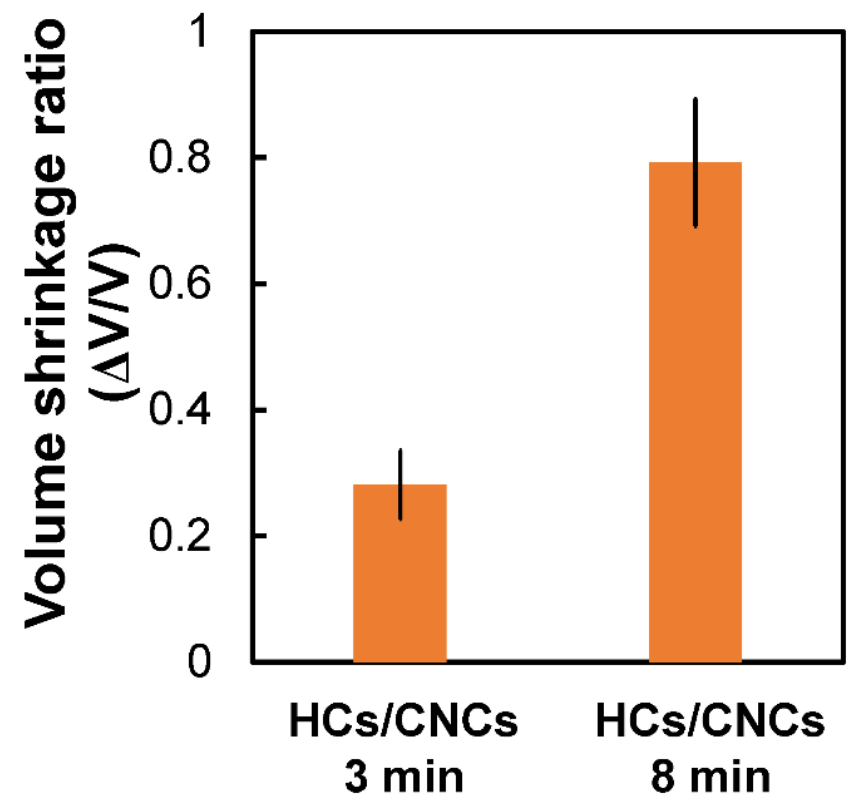

Figure S7. Volume shrinkage ratio for hydrogel container HCs/CNCs after 3 min treatment in $\mathrm{Fe}^{3+}$ solutions and bulk hydrogel $\mathrm{HCs} / \mathrm{CNCs}$ after treatment in $\mathrm{Fe}^{3+}$ solutions for 8 min.

The hydrogel container after 3 min treatment in $\mathrm{Fe}^{3+}$ solutions can only achieve limited shrinkage in volume, the shrinkage ratio was around 0.3 , but it reached about 0.8 of bulk solid samples of $\mathrm{HCs} / \mathrm{CNCs}$ with 8 min immersion time in $\mathrm{Fe}^{3+}$ solution. 


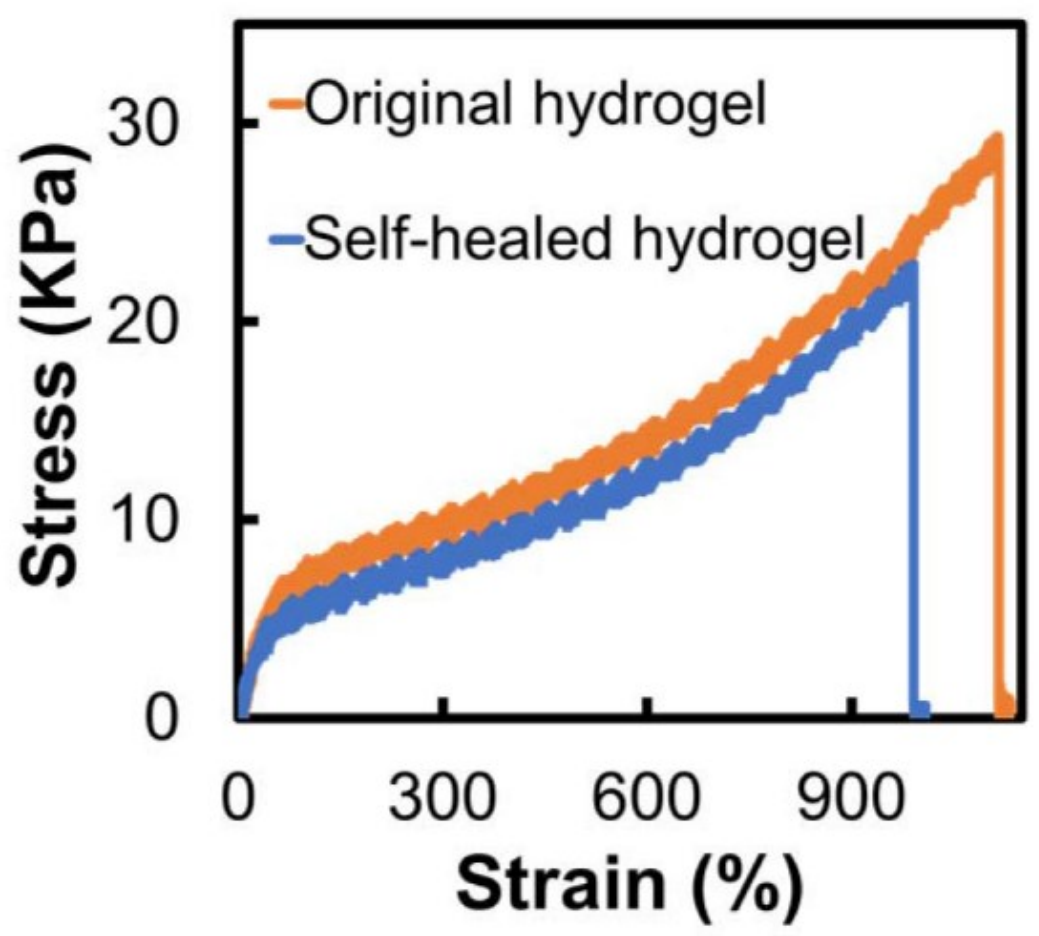

Figure S8. Tensile test stress-strain curve of original and self-healed dynamic hydrogel.

\section{Reference}

(1) Fraschini, C.; Chauve, G.; Bouchard, J., TEMPO-Mediated Surface Oxidation of Cellulose Nanocrystals (CNCs). Cellulose, 2017, 24, 2775-2790. 


\section{Publication 3}

\section{Structural Colors by Synergistic Birefringence and Surface Plasmon}

\section{Resonance}

Xiaojie Wang, ${ }^{1}$ Dan Xu, ${ }^{1}$ Bea Jaquet, ${ }^{2}$ Yang Yang,,${ }^{1,3}$ Jiaxiu Wang, ${ }^{1}$ Heqin Huang, ${ }^{1}$ Ye Chen, ${ }^{4}$

Christoph Gerhard ${ }^{2}$ and Kai Zhang*1

${ }^{1}$ Wood Technology and Wood Chemistry, Department of Wood Technology and Wood-based Composites, Georg-August-University of Göttingen, Büsgenweg 4, D-37077 Göttingen, Germany

${ }^{2}$ University of Applied Sciences and Arts, Faculty of Engineering and Health, Von-OssietzkyStraße 99, 37085 Göttingen, Germany

${ }^{3}$ State Key Laboratory of Pulp and Paper Engineering, South China University of Technology, Guangzhou, 510640, P. R. China

${ }^{4}$ State Key Laboratory for Modification of Chemical Fibers and Polymer Materials, College of Materials Science and Engineering, Donghua University, Shanghai, 201620, P. R. China

*Corresponding author Email: kai.zhang@uni-goettingen.de

Manuscript 


\section{ABSTRACT}

One-dimensional nanomaterials including cellulose nanocrystals (CNCs) and gold nanorods (GNRs) are widely used in optical materials due to their respective inherent features: birefringence with accompanying light retardation and surface plasmon resonance (SPR). Herein, we successfully combine these properties of both nanorods to generate synergistic and readily tunable structural colors in hybrid composite polymer films. CNCs and GNRs are embedded either in the same or in separate films after unidirectional alignment in dynamic hydrogels. By synergistically leveraging CNCs and GNRs with diverse amounts in hybrid films or stacked separate films, wide-ranging structural colors are obtained - far beyond those from films solely with aligned CNCs or GNRs. Higher GNRs contents enhance light absorption at $520 \mathrm{~nm}$ with promoted magenta colors, while more CNCs affect the overall phase retardation with light absorption between 400 and $700 \mathrm{~nm}$ between crossed polarizers. Moreover, adjusting the angles between films solely with CNCs or GNRs via a stacking/rotating technique successively manipulated colors with flexible film combinations. By rotating the films with aligned GNRs $\left(0-180^{\circ}\right)$, light absorption can traverse from $\sim 500$ to $650 \mathrm{~nm}$. Thus, tuning the adjustable synergism of birefringence of CNCs and SPR of GNRs provides great potential for structural colors, which enlightens inspirations for designing functional optical materials.

KEYWORDS: structural colors, cellulose nanocrystals (CNCs), gold nanorods (GNRs), birefringence, surface plasmon resonance (SPR) 
Artificial structural colors originating from resonant interactions between visible light and constructed nanostructures are promising in designing optical materials with non-fading and vibrant colors. ${ }^{1}$ Artificial nanostructures can be used to manipulate the optical fields by affecting birefringence, structural resonances, or their hybrid combinations, in order to change the amplitude, phase and polarization in subwavelength range of light and thus colors. All these optical behaviors provide the possibility to construct structural color materials with various fascinating applications, such as optical data storages, information encryption, biochemical sensing and display devices. ${ }^{2-6}$

Common methods to fabricate structural colors are implemented by constructing periodic nanostructures with distinctly shaped nanoparticles, which can alter the optical field within subwavelength to give diverse visible colors. ${ }^{7-9}$ In this scope, rod-like nanoparticles with distinct optical properties from their inherent anisotropy can be applied to construct optical materials, such as CNCs and GNRs. ${ }^{10,11}$ As an example of bio-based anisotropic nanoparticles, CNCs can be integrated into structural color materials due to their optical birefringence. ${ }^{10,12,13}$ GNRs as the widely applied precious nanoparticles can manipulate the optical field by altering their intriguing anisotropic SPR, which directly leads to visible light absorption. These inherently distinct optical mechanisms allow orthogonal strategies to construct structural color materials through successive interference and interaction with light. The macroscopic manifestation of these optical properties of anisotropic CNCs and GNRs in synthetic materials largely relies on their ordered arrangement. 
A few approaches have been demonstrated to align CNCs or GNRs in polymer matrices, such as by using magnetic field, electric field, shear force or templates with well-aligned hole array. ${ }^{14-18}$ Most of the approaches require significant energy consumption and are not compatible with polymer matrices. Although some studies attempted to co-align CNCs and GNRs in polymer matrices for structural colors, it still lacks the wide tunability of structural colors. ${ }^{19,20}$ To our best knowledge, research on the structural color control with combined birefringence of CNCs and SPR of GNRs in polymer materials based on their distinct properties has still been less explored. One major challenge is the lack of robust and compatible methods to embed ordered CNCs and GNRs in uniform polymer materials. Moreover, the successful preparation of such materials with arranged CNCs and GNRs will provide platforms including both optical mechanisms for specific optical features.

\section{RESULTS AND DISCUSSION}

In our previous work, based on the thixotropy of highly dynamic hydrogels, ${ }^{21}$ we achieved the well-controlled alignment of CNCs in polymer films from dynamic hydrogels. This provided an appropriate method to investigate the structural colors of polymer films with multiple types of ordered anisotropic nanoparticles, such as GNRs and CNCs (Figure 1a). The successful preparation of $\mathrm{CNCs} / \mathrm{GNRs}$ hybrid composite films is schematically illustrated in Figure $1 \mathrm{~b}$. The hybrid dynamic hydrogels were synthesized via in situ polymerization of acrylamide with phenylboronic acid/catechol complexes (PBA/DMA complexes) crosslinkers in alkaline buffer solutions with the presence of CNCs and/or GNRs (Figure S1), based on the optimized 
conditions from our previous study. ${ }^{21}$ After the preparation, obtained dynamic hydrogels were stretched and further air-dried to generate hybrid nanocomposite films with anisotropically aligned CNCs and GNRs.

In detail, CNCs with length of 100-200 nm and diameter of 10-20 nm were prepared via 2,2,6,6tetramethyl-1-piperidinyloxy (TEMPO)-mediated oxidation (Figure S2). GNRs with an average aspect ratio of $6.5 \pm 0.5$ (Figure S3a) and a negative surface potential of $-34.2 \mathrm{mV}$ (Figure S3b) were synthesized according to a previously reported method. ${ }^{22} \mathrm{CNCs}$ and GNRs were both synthesized with negatively charged surface to avoid the aggregation of both nanorods. Ultraviolet-visible-near-infrared (UV-Vis-NIR) extinction spectra of aqueous GNRs dispersions displayed two clear absorption peaks at around 520 and $1150 \mathrm{~nm}$ corresponding to transverse surface plasmon resonance excitation (T-SPR) and longitudinal surface plasmon resonance excitation (L-SPR), respectively (Figure S4). ${ }^{22}$ After the addition of each component in alkaline hydrogel precursor solutions, GNRs (1 wt $\%)$ were well dispersed with the presence of CNCs (1 wt \%), as shown by the polarized optical microscopy (POM) image (Figure S5a). After the polymerization, CNCs and GNRs in dynamic hydrogels were aligned during the uniaxial stretching and subsequent air-drying process. GNRs in unstretched hybrid films were randomly dispersed, whereas they were oriented in stretched hybrid films along the stretching direction according to scanning electron microscopy (SEM) images (Figures 1c and S5b). As well, the orientation of $\mathrm{CNCs}$ within the composite films has been proved using synchrotron X-ray analysis in our previous studies. ${ }^{21}$ 
a
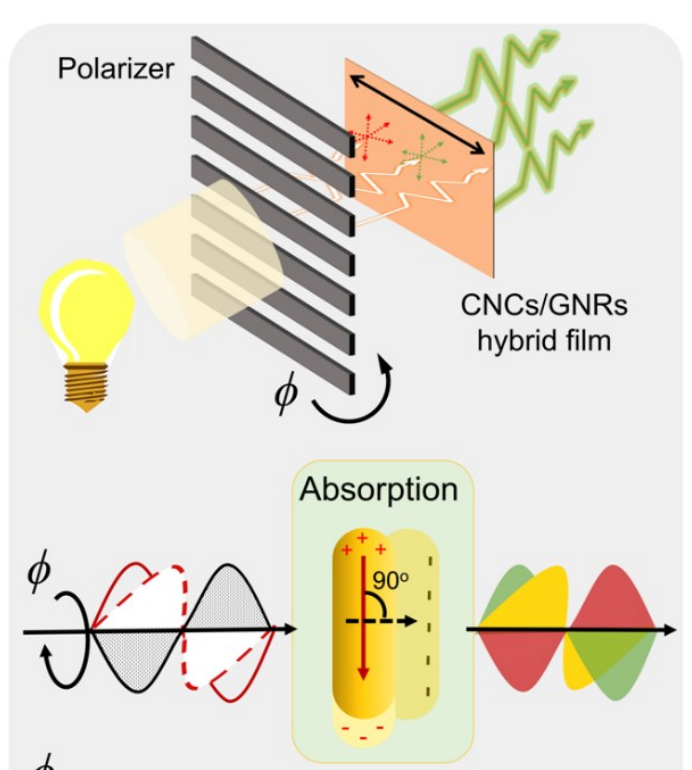

$\phi$

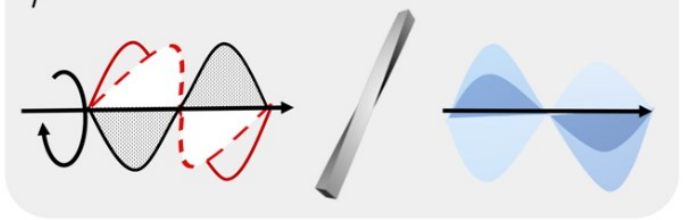

\section{d}

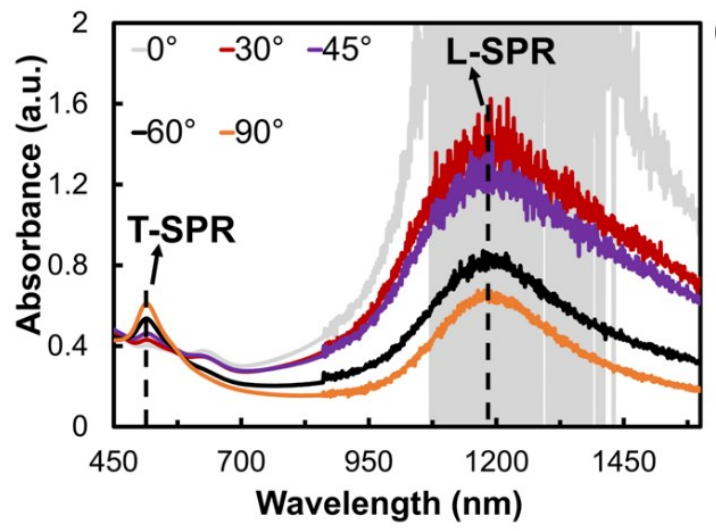

b
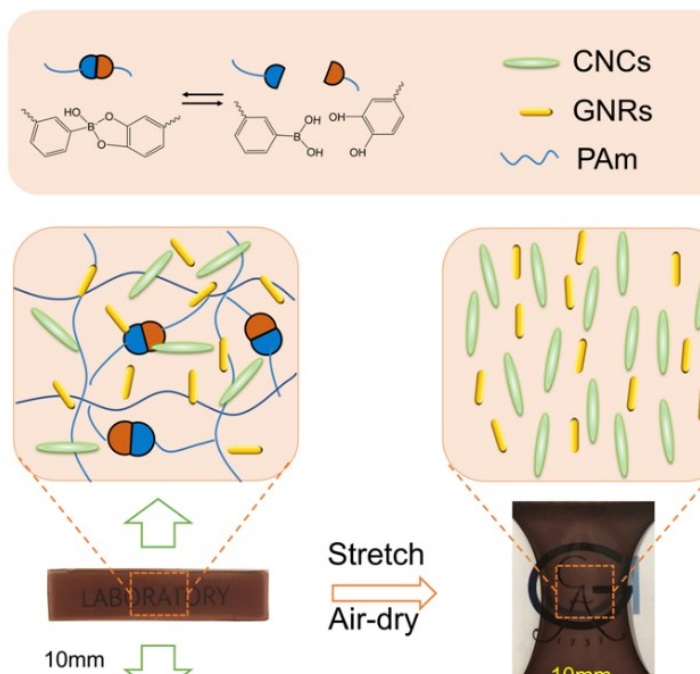

C

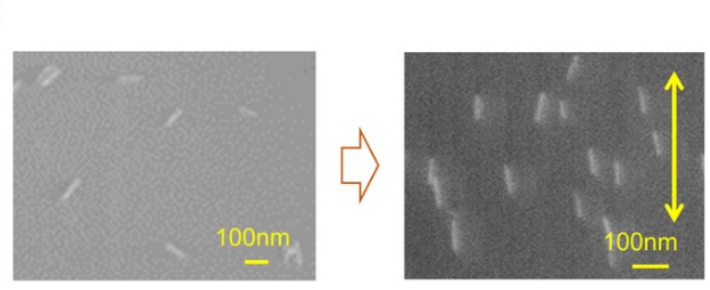

\section{e}

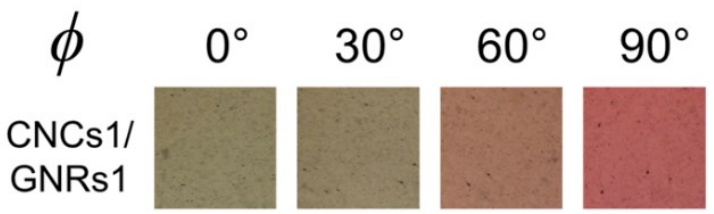

CNCs1/

GNRs2
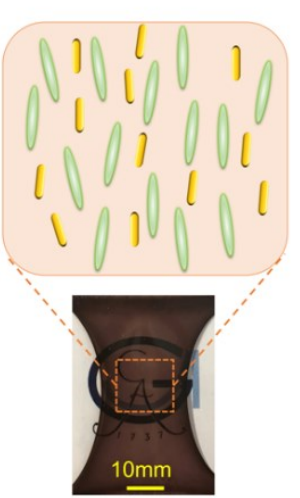

$\underset{\text { iir-dry }}{\stackrel{\text { tretch }}{\vec{y}}}$

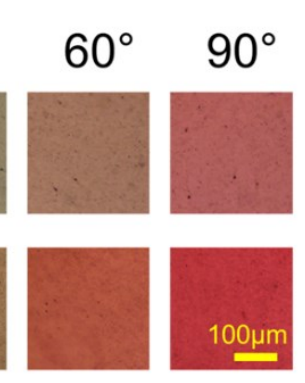

Figure 1. Fabrication of CNCs/GNRs hybrid composite films and the SPR regulation

within hybrid films. (a) Schematic illustration for the SPR regulation via varying light vibrating direction and the interference color induced by $\mathrm{CNCs}$ by rotating polarizer with a rotating angle $\phi\left(\phi=0^{\circ}\right.$ means that electric (E) vector of light is parallel to stretching direction).

Black arrow in the composite film represents stretching direction. (b) Schematic illustration for 
preparing CNCs/GNRs hybrid composite films. (c) Representative SEM images of GNRs embedded in air-dried hybrid films without (left panel) and with stretching (right panel). Yellow arrow represents stretching direction. (d) UV-Vis-NIR spectra of CNCs1/GNRs1 hybrid films with the gradual change of SPR with varying $\phi$ from $0^{\circ}$ to $90^{\circ}$. The high noise level at $0^{\circ}$ was due to the strong absorbance. (e) POM images of colors from CNCs1/GNRsX hybrid films with varying $\phi$ from $0^{\circ}$ to $90^{\circ} . \mathrm{X}=1$ or $2 \mathrm{wt} \%$ of GNRs in the original hydrogel precursor solutions.

As illustrated in Figure 1a, the SPR of GNRs depended on the light vibrating direction with selective excitation of L-SPR and T-SPR in a trading off rule. The angle between light vibrating directions to the longitudinal axis of GNRs will be changed by varying the angle between the polarizer and composite films in the stretching direction (defined as $\phi$ ). At $\phi=0^{\circ}$, L-SPR is excited with light vibrating along the longitudinal axis of GNRs, whereas light vibrating perpendicular to the longitudinal axis can excite T-SPR at $\phi=90^{\circ}$. The rotation of polarizer leads to light absorption at selective wavelength with tunable intensities due to modified combinations of T-SPR and L-SPR. ${ }^{11}$ Because of the aligned GNRs in the hybrid polymer films, the SPR of single GNRs was transferred to CNCs/GNRs hybrid films in the macroscale by using only one polarizer, without the interference of birefringence from CNCs. As shown in the UV-Vis-NIR spectra of CNCs1/GNRs1 hybrid films measured with one polarizer (Figure 1d), the absorption intensity at wavelength $520 \mathrm{~nm}$ (T-SPR) largely increased, while that at the wavelength of $1150 \mathrm{~nm}$ (L-SPR) greatly reduced with rising $\phi$ from $0^{\circ}$ to $90^{\circ}$. For our naked 
eyes, the $520 \mathrm{~nm}$ light is corresponding to green colors. Therefore, its complementary color as magenta was enhanced due to the absorption of light at around $520 \mathrm{~nm}$ and its intensity increased with higher $\phi$ from $0^{\circ}$ to $90^{\circ}$ (Figure 1e). The visible colors became brighter with increasing amounts of GNRs in CNCs/GNRs films (Figure 1e).

In addition to the effect of aligned GNRs on optical fields due to tunable light absorption intensities at around $520 \mathrm{~nm}$ and $1150 \mathrm{~nm}$ based on modified SPR, the synergistic effect of ordered GNRs and CNCs on the structural colors of hybrid CNCs/GNRs films was further investigated. The interactions of birefringence from CNCs and SPR from GNRs with incident light waves happen simultaneously, when the polarized light is irradiated onto hybrid films, as schematically illustrated in Figure 2a. This synergistic effect generated the tunable structural colors, when $\mathrm{CNCs} / \mathrm{GNRs}$ hybrid films were placed with $45^{\circ}$ against crossed polarizer/analyzer. In order to classify their respective roles of birefringence and SPR in generating these structural colors, we analyzed the structural colors of the hybrid films with fixed content of GNRs or CNCs, while changing the amount of the other nanorod. 
a

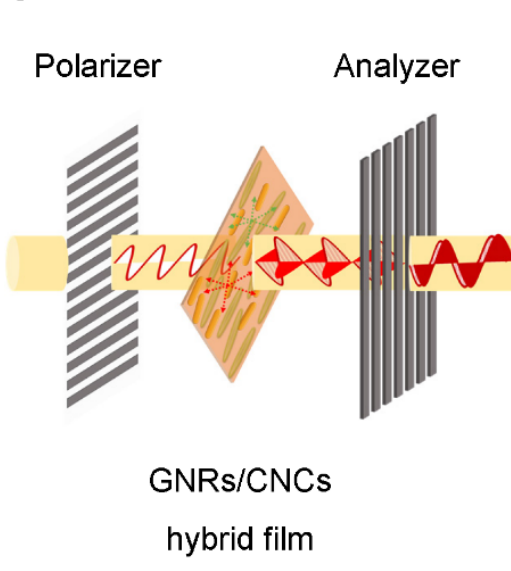

b

GNRs1/CNCs Y
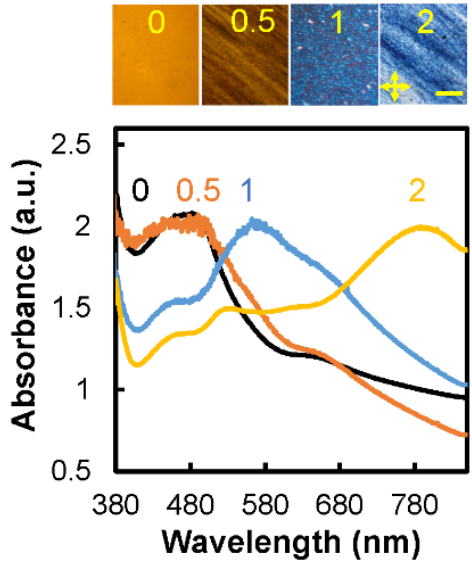

C CNCs1/GNRsX
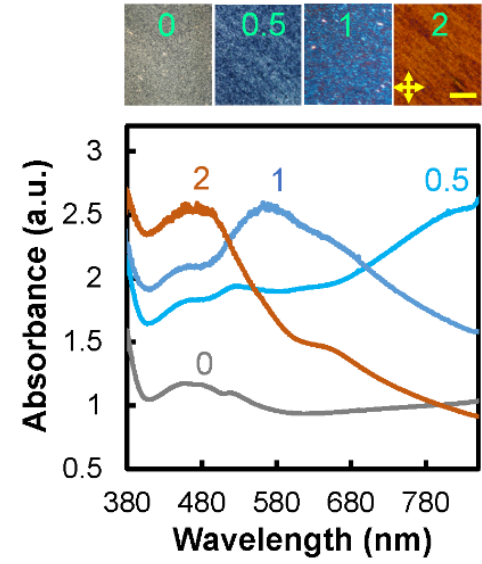

Figure 2. Integration of birefringence and SPR in CNCs/GNRs hybrid films. (a) Schematic illustration of synergistic color adjustment between crossed polarizers. CNCs/GNRs hybrid films were placed at $45^{\circ}$ against crossed polarizer/analyzer. (b-c) POM images and UV-Vis spectra of CNCs/GNRs hybrid films measured between crossed polarizers with increasing contents of CNCs $(\mathrm{Y}=0-2 \mathrm{wt} \%$ in initial hydrogel precursor solutions) or GNRs $(\mathrm{X}=0-2 \mathrm{wt} \%$ in initial hydrogel precursor solutions), respectively. The absorbance was recorded by measuring the transmitting light through the polarizer, films and analyzer. Scale bars: $250 \mu \mathrm{m}$.

With fixed GNRs content at $1 \mathrm{wt} \%$ in initial hydrogel precursor solutions (roughly $5.9 \mathrm{wt} \%$ in dried films, Table S1), structural colors varied from orange to blue with increasing CNCs concentration from 0 to $2 \mathrm{wt} \%$ (Figure $2 \mathrm{~b}$ ). Furthermore, the UV-Vis spectra of hybrid films were measured between crossed polarizers with the films placed $45^{\circ}$ against the crossed polarizer/analyzer. The wavelengths of absorbance peaks in the visible region of UV-Vis spectra measured between crossed polarizers were red-shifted from $\sim 480 \mathrm{~nm}$ to around $780 \mathrm{~nm}$ (Figure 2b), which corresponded to observed structural colors from orange to blue. This is 
because color manipulation from birefringence was primarily originating from modified light retardations of CNCs, which are accumulated phase difference between light vibrating perpendicularly and parallel to the films with aligned CNCs. Films with higher CNCs concentrations would enhance light retardation due to the presence of more CNCs along the light propagation pathway, leading to the shift of absorbance peaks (Figure S6). ${ }^{10,21}$ These hybrid films still exhibited relatively constant SPR despite of distinct amounts of CNCs due to fixed GNRs content, when observed with only one polarizer (Figure S7). Therefore, manipulation of structural colors was realized by varying the birefringence and accompanying accumulated phase retardation of CNCs with distinct amounts between 0 and $2 \mathrm{wt} \%$.

Furthermore, with fixed CNCs content of $1 \mathrm{wt} \%$ and increasing GNRs contents from 0 to 2 $\mathrm{wt} \%$, the maximal wavelength of light absorption was shifted from $\sim 860 \mathrm{~nm}$ to around $490 \mathrm{~nm}$ (Figure 2c). The visible colors of obtained films were red-shifted from light yellow to orange red due to modified light absorption by varying GNRs contents from 0 to $2 \mathrm{wt} \%$. This is because of the large increase of T-SPR of GNRs at $520 \mathrm{~nm}$ with higher GNRs contents, while L-SPR at $1150 \mathrm{~nm}$ showed only very slight enhancement (Figure S8). Therefore, more GNRs largely contributed to regulating the intensity of light absorption at wavelength of $\sim 520 \mathrm{~nm}$ that lies in green light region as shown above (Figure 1d and 1e) and enhance magenta colors in the synergistic structural colors.

Because the structural colors between polarizers do not originate from polymer matrix due to the fast relaxation of polymer chains during stretching and drying, ${ }^{21}$ but rather from ordered CNCs and GNRs within the composite films. The physical location of these nanorods should 
not affect the resulting structural colors, as long as their organizations are equal. Therefore, overall appeared colors from piled individual films with only CNCs (f-CNCs) or only GNRs (f-GNRs) as separate films should be equivalent to those of hybrid films with both CNCs/GNRs nanorods in the same films. In comparison, the separation of CNCs and GNRs in two separate films also provides the possibility to modularly construct the structural colors and to exactly elucidate their functions. By stacking f-GNRs and f-CNCs films along the stretching direction and placing them $45^{\circ}$ against the crossed polarizer/analyzer with f-GNRs film facing the polarizer, diverse nearly equal colors as those of CNCs/GNRs hybrid films with the same contents of both nanorods were obtained (Figure 2b, 2c and 3a). For instance, a CNCs 1/GNRs1 hybrid film had similar absorption spectrum and exhibited highly similar royal blue colors as those of piled films with the same contents of CNCs (f-CNCs1) and GNRs (f-GNRs1) (Figure S9). Moreover, these structural colors gradually evolved into red colors of diverse intensities by increasing GNRs amount at constant CNCs amounts (Figure 3a). This should be partially due to the enhanced light absorption at $\sim 520 \mathrm{~nm}$ (Figure 3b, left panel), but negligible intensity changes at around $1150 \mathrm{~nm}$ (Figure S10). Higher light absorption at about $520 \mathrm{~nm}$ resulted in enhanced structural magenta color (Figure 2c). In combination with the effect of birefringence by stacking f-CNCs5 film and f-GNRs films with increasing GNRs contents from 0 to $3 \mathrm{wt} \%$, wavelengths of light absorption peaks also exhibited a steady blue-shift from $\sim 665 \mathrm{~nm}$ to 510 $\mathrm{nm}$, corresponding to the change of more blue-green to red colors (Figure 3b, right panel). 
a

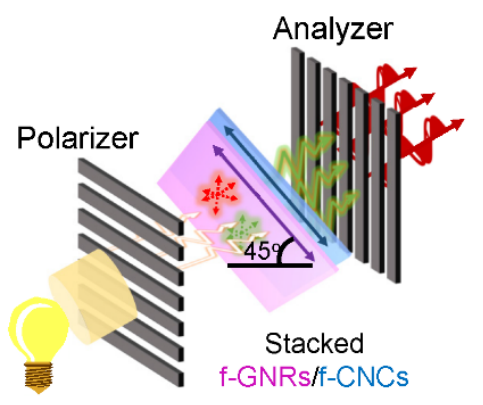

b Contribution of GNRs

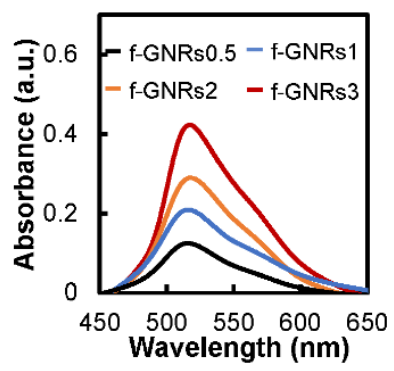

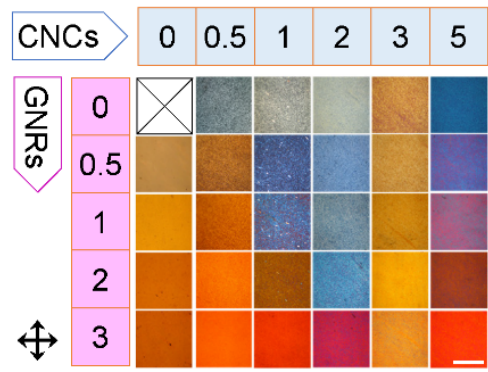

d

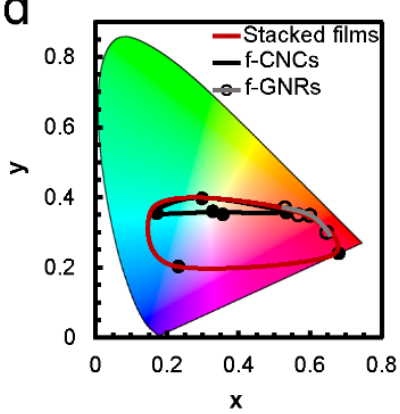

C Contribution of CNCs

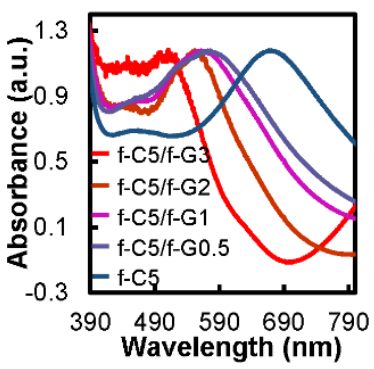

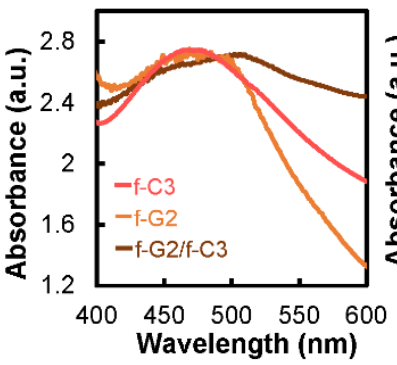

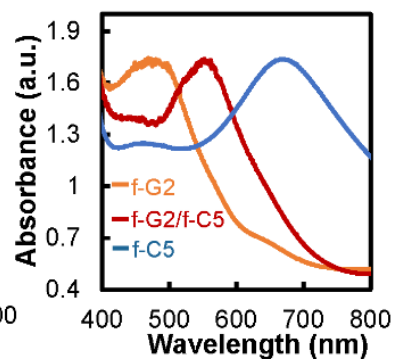

Figure 3. Color manipulation via stacking composite films f-GNRs and f-CNCs. (a)

Schematic illustration for the detection of stacked films and vivid colors generated from various combinations of f-CNCs and f-GNRs films. f-GNRs and f-CNCs films were overlapped along the stretching direction and then placed $45^{\circ}$ against the crossed polarizer/analyzer with f-GNRs film facing the polarizer. Black arrows in the composite films represent the stretching direction. Scale bar: $400 \mu \mathrm{m}$. (b) UV-Vis spectra of f-GNRs composite films measured without analyzer (left panel) and stacked f-CNCs5/f-GNRsX composite films measured between crossed polarizer/analyzer (right panel). $\mathrm{X}=0,0.5,1,2$ or 3 . (c) UV-Vis spectra of $\mathrm{f}-\mathrm{CNCs} 3$ film and stacked f-GNRs2/f-CNCs3 in left panel as well as f-CNCs5 film and f-GNRs2/f-CNCs5 films in right panel measured between crossed polarizer/analyzer. The spectrum of the film f-GNRs2 (f-G2) measured with one polarizer is shown as reference. $\mathrm{f}-\mathrm{C}$ : abbreviation for $\mathrm{f}-\mathrm{CNCs}, \mathrm{f}-\mathrm{G}$ : abbreviation for f-GNRs. (d) Evolution of structural colors of stacked films in comparison to individual f-CNCs and f-GNRs films in CIE chart. 
Different than the SPR effect of GNRs, the structural colors in f-CNCs films were correlated to accumulated light retardation originating from aligned $\mathrm{CNCs}$ with diverse contents. Therefore, light retardation and interference colors were further altered by increasing $\mathrm{CNCs}$ contents (Figure S6). These f-CNCs films with increasing initial CNCs concentrations from 0 to $5 \mathrm{wt} \%$ showed versatile structural colors from grey over light yellow to blue-green colors (Figure 3a, right panel). The UV-Vis spectra of the f-CNCs films with initial 2, 3 and $5 \mathrm{wt} \%$ showed an obvious change of the main absorption peak between $470 \mathrm{~nm}$ to $665 \mathrm{~nm}$, corresponding to the color development in Figure 3a (Figure S6, right panel).

By stacking diverse f-CNCs films with the f-GNRs2 film, various structural colors emerged (Figure 3a, right panel). For instance, structural colors appeared from stacked f-GNRs2/fCNCs3 was orange with an overall light absorption peak at around $500 \mathrm{~nm}$, which was redshifted compared to the light absorption peak of f-CNCs3 at $477 \mathrm{~nm}$ (Figure 3c). Piled fGNRs2/f-CNCs5 films displayed red-violet color with the absorption peak at $550 \mathrm{~nm}$, which was blue-shifted from the absorption peak at $665 \mathrm{~nm}$ for $\mathrm{f}-\mathrm{CNCs} 5$. These results demonstrated that the synergistic structural colors as well as wavelengths of absorption peaks for stacked fGNRs and f-CNCs films strongly depended on the interference color and locations of absorption peaks of f-CNCs. Moreover, GNRs interacted with light by altering the intensity of light absorption at wavelength $\sim 520 \mathrm{~nm}$ for the manipulation of colors, while the aligned CNCs produced periodic interference colors via light retardation.

By integrating these two mechanisms, the tunable color range was apparently enlarged owing to the synergistic effect of birefringence and SPR. As displayed in the established Commission 
Internationale de l'Éclairage (CIE) chart, the piled films covered a color range from blue through yellow-green to violet (circled by red line in Figure 3d), while single f-CNCs or fGNRs films showed limited color regulation regions (circled by black line and grey line in Figure 3d). The adjustable range of structural colors was significantly enlarged owing to the synergistic effect of birefringence and SPR, and such broad structural color range cannot be solely achieved by f-CNCs or f-GNRs. Therefore, by separately adjusting the dosages of CNCs and GNRs in two individual films, the structural colors of stacked film piles can be further manipulated in an even broader color range than embedding both nanorods in the same films (Figure 3a). Moreover, the use of separate films provided a modular fabrication method with the arbitrary combination of diverse films, e.g. by using two f-CNCs3 films and various fGNRsX films for color evolution from green to red (Figure S11). 
a

Rotating f-GNRs of stacked f-GNRs/f-CNCs
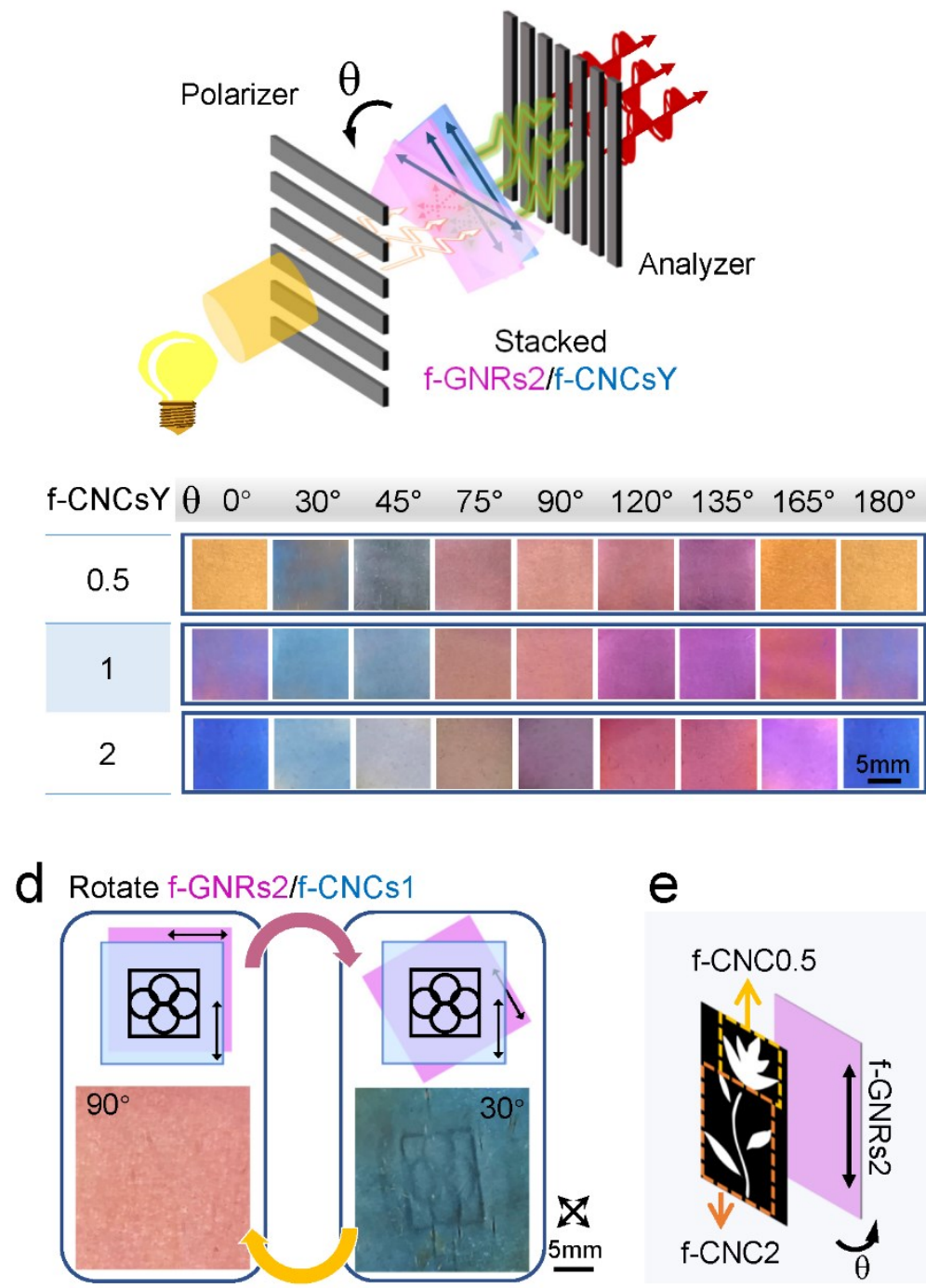

e
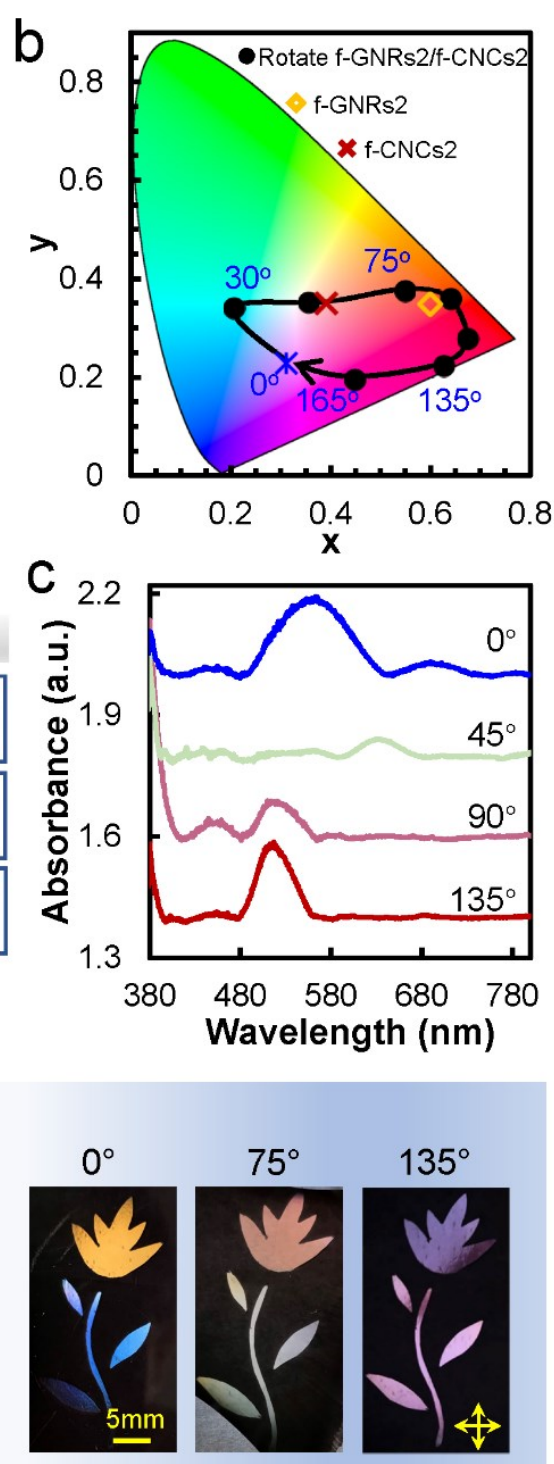

Figure 4. Tunable color manipulation via rotating f-GNRs films in stacked f-GNRs/f-

CNCs film piles. (a) Schematic illustration for the detection and digital photos of vivid colors generation by rotating f-GNRs films in the stacked film piles at a rotating angle of $\theta$ between both films ( $\theta$ representing rotating angles of $\mathrm{f}-\mathrm{GNRs}$ film related to $\mathrm{f}-\mathrm{CNCs}$ film). $\mathrm{f}-\mathrm{GNR}$ and f-CNCs composite films were stacked along stretching direction and placed $45^{\circ}$ against the crossed polarizer/analyzer with rotating f-GNRs film facing the polarizer as the initial state. Combinations of f-GNRs2 film (in pink) and diverse f-CNCsY films (in blue) were used to 
tune the colors. (b) Color evolution of $\mathrm{f}-\mathrm{GNRs} 2 / \mathrm{f}-\mathrm{CNCs} 2$ film piles by changing the rotating angle $\theta$ between both films in comparison to singlef-GNRs 2 and f-CNCs2 film in a CIE chart. (c) UV-Vis spectra of f-GNRs2/f-CNCs2 piles by rotating f-GNRs2 film with rotating angles $\theta$ between both films between $0-135^{\circ}$. (d-e) Demonstration for potential applications as (d) information encryption and (e) colorful displays based on this tunable color manipulation by rotating f-GNRs2 films at diverse rotating angles with the use of two different f-CNCs films. The pictures were taken under POM. The arrows in the composite films represent the stretching direction.

In addition to adjusting the CNCs and GNRs contents in the individual films, rotating GNRs film in the film piles demonstrates another strategy to generate a broad color range. In particular, this strategy can further provide more flexibility to tune the colors with fewer polymer films and save the preparation of polymer films with various contents of CNCs or GNRs. As illustrated in Figure 4a, f-GNRs2 and various f-CNCs composite films were stacked as described above in Figure 3. By rotating the f-GNRs2 film with a rotating angle $\theta$ between $\mathrm{f}-$ GNRs2 film and f-CNCs films of $0-180^{\circ}$, structural colors experienced successive color variation as blue, orange, red and purple (Figure 4a, bottom panel).

As shown in the CIE chart (Figure 4b), structural colors of the f-CNCs2/GNRs2 films after stacking and rotating covered a broad color range from blue purple to cyan and gradually to yellow-green and red. As comparison, the structural colors of f-CNCs2, f-GNRs2 and their piled system without rotating only appeared as separate single dots in the CIE chart, which also 
reflected the synergistic effect of these two optical mechanisms. Simply rotating the f-GNRs2 film of the stacked film piles strongly expanded the color tunability. Furthermore, the corresponding UV-Vis spectra of stacked f-GNRs2 and f-CNCs2 with various rotating angles were measured to show the absorption changes during the rotation (Figure $4 \mathrm{c}$ ). The dominating absorbance peaks can be readily controlled by rotating the f-GNRs 2 film, which was redshifted from around $560 \mathrm{~nm}$ to $638 \mathrm{~nm}\left(0^{\circ}-45^{\circ}\right)$, then blue-shifted to $517 \mathrm{~nm}\left(45^{\circ}-135^{\circ}\right)$, and eventually back to $560 \mathrm{~nm}\left(180^{\circ}\right)$. This evolution is corresponding to their sequential color change from blue-purple through cyan and red to blue-purple. This further demonstrated the flexible color regulation by adjusting the synergistic effect of SPR of GNRs and light retardation of CNCs. The light absorption at T-SPR and L-SPR of GNRs was altered by changing the incident light vibrating direction during the rotation (Figures $4 \mathrm{a}$ and $1 \mathrm{a}, 1 \mathrm{~d}$ and 1e). Because of the presence of GNRs in the individual films, f-GNRs films functioned more like a further light filter before f-CNCs films, so that the wavelengths of the incident lights after the f-GNRs film changed correspondingly. These incident lights with altered wavelengths further underwent light retardation through the f-CNCs films, leading to diverse colors. As the result, a much broader range of structural colors was achieved (Figure 4a-4b).

Based on the broad color tunability and facile flexible construction of the stacking/rotating film piles, we developed a few prototypes of $\mathrm{f}-\mathrm{CNCs} / \mathrm{f}-\mathrm{GNRs}$ films for potential applications. As demonstrated in Figure 4d, a transparent f-CNCs1 composite film with a stamped pattern was prepared from the stamped hydrogel precursor and used with f-GNRs2 film as the stacked piles. 
By stacking both films perpendicular to each other $\left(90^{\circ}\right)$, in which the absorption of T-SPR of GNRs was minimized, the stamped pattern was not visible between crossed polarizer/analyzer. The stamped pattern appeared by rotating the f-GNRs 2 film with an angle of $30^{\circ}$ to the $\mathrm{f}$ CNCs1 film, which indicated effective information encryption and readout on demand. Moreover, based on the abundant colors, good transmission and low reflection of light (Figures S12 and S13) and applicable mechanical properties (Figure S14) from diverse combinations of f-CNCs and f-GNRs films, optical films can be fabricated as tunable color display systems. As illustrated in Figure 4e, a variable color flower pattern was designed by stacking f-GNRs2 film with f-CNCs0.5 film as the flower part and with f-CNCs2 film as the stalk and leaves. With the rotating f-GNRs2 film and altering light retardation due to various CNCs contents in fCNCs films, the flower pattern exhibited distinct, adjustable color combinations. Although sophisticated and promising manipulations of plasmonic colors have been fabricated via complicated micro and nanofabrication methods, for example, the widely used physical vapor deposition to selectively coat metal layers, a general method to prepare composite polymer films with embedded nanorods with tunable morphologies and optical mechanism is still highly desired. ${ }^{19,20,23}$ In this work, we presented an effective and cost-efficient method to achieve flexible color manipulation of polymer films by integrating CNCs with birefringence and GNRs exhibiting SPR. Using this method, polymer films containing aligned GNRs and/or CNCs not only showed much wider range of adjustable structural colors than existing methods, but also can be expanded to other potential nanomaterials. With this method, these optical film systems with tunable and flexible color control provide effective platforms for the applications 
in information encryption and security systems, which have gained significant interest as security materials in the last years. ${ }^{24,25}$

\section{CONCLUSIONS}

In summary, we demonstrated the synergistic structural colors by combining the birefringence of CNCs and the SPR of GNRs within the composite films by using a facile and efficient method, which were embedded either in the same or in separate films. The birefringence of CNCs induced light retardation and the SPR of GNRs resulted to regulable light absorption around $520 \mathrm{~nm}$, which were integrated for the tunable color generation by the hybrid composite films. With these two orthogonally polarization-dependent responses of the GNRs and CNCs that show two different combinable color mechanisms, the color tunability was significantly enlarged by hybrid films or stacked f-CNCs/f-GNRs films owing to the synergistic effect of birefringence and SPR, and such broad structural color range cannot be solely achieved by fCNCs or f-GNRs. Moreover, a facile stacking/rotating technique led to an even broader color manipulation with modular stacking combinations. Based on the effective and readily color manipulation, these optical films could be potentially used as display devices and optical encryption, giving inspirations for designing sophisticated photonic functional materials.

\section{METHODS}

Preparation of CNCs. CNCs were synthesized via the TEMPO-mediated oxidation of MCC according to previous report. ${ }^{26}$ After dialysis, the obtained CNCs were stored at room 
temperature for $24 \mathrm{~h}$ and then the precipitated large particles were removed. The upper stable suspension was stored in $4{ }^{\circ} \mathrm{C}$ fridge for future use.

Synthesis of GNRs. We adopted the seed-mediated growth method. ${ }^{22}$ The seed solution for GNRs was prepared as follows: $5 \mathrm{~mL} \mathrm{HAuCl} 4 \cdot 3 \mathrm{H}_{2} \mathrm{O}$ solution $(0.5 \mathrm{mM})$ was mixed with $5 \mathrm{~mL}$ CTAB $(0.2 \mathrm{M})$ solution in a $20 \mathrm{~mL}$ glass bottle, then, $0.6 \mathrm{~mL}$ fresh prepared $\mathrm{NaBH}_{4}$ solution $(0.01 \mathrm{M})$ was diluted to $1 \mathrm{~mL}$ and was immediately injected to the $\mathrm{HAuCl}_{4} / \mathrm{CTAB}$ mixed solution under vigorous stirring at $1200 \mathrm{rpm}$. The seed solution color transferred from yellow to brownish yellow and the stirring was stopped after $2 \mathrm{~min}$. The seed solution was aged at room temperature undisturbed for $30 \mathrm{~min}$ before use.

The growth solution for GNRs was prepared as follows: $3.5 \mathrm{~g} \mathrm{CTAB}$ and $0.617 \mathrm{~g} \mathrm{NaOL}$ were dissolved in $125 \mathrm{~mL}$ warm deionized water $\left(\sim 50^{\circ} \mathrm{C}\right)$ in a $500 \mathrm{~mL}$ flask. Then, the solution was cooled down to $30{ }^{\circ} \mathrm{C}$. After that, $12 \mathrm{~mL} \mathrm{AgNO}_{3}$ solution $(4 \mathrm{mM})$ was added and the mixture was left undisturbed for $15 \mathrm{~min}$ at $30{ }^{\circ} \mathrm{C}$. Following that, $125 \mathrm{~mL} \mathrm{HAuCl} \cdot 3 \mathrm{H}_{2} \mathrm{O}$ solution (1 $\mathrm{mM}$ ) was added under stirring $(700 \mathrm{rpm})$. The solution became colorless after $90 \mathrm{~min}$ and 2.7 $\mathrm{mL} \mathrm{HCl}$ solution (37\%) was added under magnetic stirring for $15 \mathrm{~min}$ (400 rpm). Afterwards, $0.625 \mathrm{~mL}$ L-ascorbic acid solution $(0.064 \mathrm{M})$ was injected under vigorous stirring (1200 rpm) for 30 s. $0.4 \mathrm{~mL}$ seed solution was injected to the growth solution with stirring (1200 rpm) for $30 \mathrm{~s}$. Finally, the solution was placed without disturbance at $30^{\circ} \mathrm{C}$ for $12 \mathrm{~h}$ for GNRs growth. The obtained CTAB-capped GNRs dispersion was centrifuged at $14000 \mathrm{rpm}$ for $10 \mathrm{~min}$ to remove excess $\mathrm{CTAB}$ and washed with water for a few times. Then, the washed CTAB-capped GNRs were redispersed in $30 \mathrm{~mL}$ water. 
Afterwards, $20 \mathrm{~mL}$ of original CTAB-capped GNRs were centrifuged and the supernatant was removed. Then, the CTAB-capped GNRs were redispersed in $20 \mathrm{ml}$ PSS solution $(10 \mathrm{mg} / \mathrm{mL})$ and the dispersion was placed undisturbed for $1 \mathrm{~h}$ to achieve the ligand exchange. ${ }^{27}$ Afterwards, the GNRs dispersion was washed with DI water for 3 times, then the PSS-capped GNRs were obtained and redispersed in $20 \mathrm{~mL}$ DI water. All the samples used GNRs were referred to the PSS-capped GNRs.

Preparation of CNCs/GNRs hybrid composite films. The CNCs/GNRs composite films were prepared via uniaxial mechanical stretching and further air-drying of dynamic hydrogels. The dynamic hydrogels were synthesized by in situ polymerization of acrylamide with PBA/DMA complexes as dynamic crosslinkers in the presence of mixture of CNCs and GNRs. In detail, $2 \mathrm{M}$ acrylamide and $25 \mathrm{mM}$ DMA/PBA were dissolved in the $\mathrm{pH} 10$ buffer solution with $0.5 \mathrm{wt} \%$ LAP as photo initiator. Certain amount of CNCs and GNRs were centrifuged, mixed and redispersed into the above solution. The mixtures were degassed with $\mathrm{N}_{2}$ for 3 min to remove the dissolved oxygen. Then, they were injected into rectangle-shaped molds with the dimension of $60 \mathrm{~mm} \times 20 \mathrm{~mm} \times 2 \mathrm{~mm}$. Following that, this system was exposed to UV light $(300-400 \mathrm{~nm}, 8 \mathrm{~W})$ for $30 \mathrm{~min}$ to finish the polymerization. In hybrid films with both CNCs and GNRs, the loading amount of CNCs and GNRs were tuned between 0-2 wt $\%$ compared to the weight of the whole hydrogel. Obtained composite hydrogels were stretched to specific length at a speed of $6 \mathrm{~mm} / \mathrm{min}$ and further air-dried at $20^{\circ} \mathrm{C}$ under the surroundings with a relative humidity of $65 \%$ for over $12 \mathrm{~h}$ until constant weight. The air-dried hybrid films containing both nanorods were referred to as "CNCs/GNRs". Composite films with only GNRs were named as "f-GNRsX", while those only with CNCs were referred to as "f-CNCsY", where 
$\mathrm{X}$ and $\mathrm{Y}$ referred to the weight percentage of GNRs and CNCs in the initial hydrogel solutions, respectively.

For $\mathrm{f}-\mathrm{CNCs}$, CNCs concentration was tuned from 0 to $5 \mathrm{wt} \%$. For $\mathrm{f}-\mathrm{GNRs}$, GNRs concentration was tuned from 0 to $2 \mathrm{wt} \%$. f-GNRs3 is a sample via piling f-GNRs1 and f-GNRs2 films.

Preparation of patterned f-CNCs1 films. The rectangle-shaped dynamic hydrogel with CNCs concentration of $1 \mathrm{wt} \%$ was synthesized (dimensions: $60 \mathrm{~mm} \times 20 \mathrm{~mm} \times 2 \mathrm{~mm}$ ), and uniaxially stretched with the elongation ratio of 6 . Then, a geometric stamp was pressed onto this stretched f-CNCs1 hydrogel. After the following air-drying process, a transparent f-CNCs1 composite film was obtained with the stamped pattern.

\section{CHARACTERIZATION METHODS}

The uniaxial stretching of dynamic hydrogels was performed with a Z3 micro tensile test machine from Grip-Engineering Thümler GmbH. The shape and size of GNRs were characterized with transmission electron microscopy (TEM), the specimens were prepared from its dispersion in water of $0.1 \mathrm{wt} \%$. The TEM observation was conducted with a CM 12 Transmission Electron Microscope (Philips, Netherland). Scanning electron microscopy (SEM) were applied to observe the alignment of GNRs in dried films with LEO Supra-35 HighResolution Field Emission Scanning Electron Microscope (Carl Zeiss AG, Germany). The optical microscopy observation and retardation measurement of films were performed with Eclipse E600 Polarizing Microscope from Nikon, or LV100 Polarizing Microscope from Nikon with Bereck compensator. The UV-Vis-NIR spectra of GNRs dispersion and composite films were measured from 450-1400 nm by UV-Vis-NIR spectrophotometer Cary 5000 (Varian Inc., 
Germany) with two detachable Glan Thompson Polarizers (wavelength range: 220-3500 nm). Zeta potential measurement was performed on a Zetasizer Nano ZS (Malvern Instruments Ltd., UK), the CTAB-capped GNRs and PSS-capped GNRs suspensions were diluted to a concentration of $\sim 0.5 \mathrm{mg} / \mathrm{mL}$ with DI water. $1 \mathrm{~mL}$ of GNRs suspension in DTS1070 disposable folded capillary cell was used for the measurements.

\section{ASSOCIATE CONTENT}

\section{Supporting Information}

The Supporting Information is available free of charge via the Internet at http://pubs.acs.org.

Supporting Figures S1-S14, Table S1 and Materials, Methods.

\section{AUTHOR INFORMATION}

\section{Corresponding Author}

*Email: kai.zhang@uni-goettingen.de

\section{Notes}

The authors declare no competing financial interest.

\section{ACKNOWLEDGEMENTS}

K.Z. thanks the Federal Ministry for Economic Affairs and Energy (BMWi) and the Ministry for Science and Culture of Lower Saxony (MWK), Germany for the financial support of 
WIPANO project (FKZ03THW05K14). K.Z. thanks Ministry of Science and Culture of Lower Saxony, Germany for the financial support of the program "Material Science Wood". X.J.W., D.X. and J.X.W. thank China scholarship council (CSC) for financial support. Y.Y. thanks the China Postdoctoral Science Foundation (2019M652905). We thank Ms. Huan Liu, Mr. Hua Zhang and Mr. Yawen Yao for supporting the SEM measurements. We also thank Dr. Serhiy Demeshko, Dr. Jürgen Ihlemann and Mr. Lukas Janos Richter for the support of UV-Vis-NIR tests. We thank Dr. Hantke Kristian from Max Planck Institute for Dynamic and Selforganization for the support of POM tests.

\section{REFERENCES}

(1) Zhu, X.; Yan W.; Levy, U.; Mortensen, N.; Kristensen, A. Resonant Laser Printing of Structural Colors on High-Index Dielectric Metasurfaces. Sci. Adv. 2017, 3, e1602487.

(2) Zhang, Q.; Xia, Z.; Cheng, Y.-B; Gu, M. High-Capacity Optical Long Data Memory Based on Enhanced Young's Modulus in Nanoplasmonic Hybrid Glass Composites. Nat. Commun. 2018, 9, 1183.

(3) Xu, M.; Ma, C.; Zhou, J.; Liu, Y.; Wu, X.; Luo, X.; Li, W.; Xu, H.; Wang, Y.; Chen, Z.; Li, J.; Liu, S. Assembling Semiconductor Quantum Dots in Hierarchical Photonic Cellulose Nanocrystal Films: Circularly Polarized Luminescent Nanomaterials as Optical Coding Labels. J. Mater. Chem. C 2019, 7, 13794-13802.

(4) Liu, B.; Lu, X.; Qiao, Z.; Song, L.; Cheng, Q.; Zhang, J.;Zhang, A.; Huang, Y.; Chen, T. pH and Temperature Dual-Responsive Plasmonic Switches of Gold Nanoparticle Monolayer Film for Multiple Anticounterfeiting. Langmuir 2018, 34, 13047-13056. 
(5) Li, W.; Xu, M.; Ma, C.; Liu, Y.; Zhou, J.; Chen, Z.; Wang, Y.; Yu, H.; Li, J.; Liu, S. Tunable Upconverted Circularly Polarized Luminescence in Cellulose Nanocrystal Based Chiral Photonic Films. ACS Appl. Mater. Interfaces 2019,11, 23512.

(6) Wang, Y.; Aurelio, D.; Li, W.; Tseng, P.; Zheng, Z.; Li, M.; Kaplan, D. L.; Liscidini, M.; Omenetto, F. G. Modulation of Multiscale 3D Lattices through Conformational Control: Painting Silk Inverse Opals with Water and Light. Adv. Mater. 2017, 29, 201702769.

(7) Sano, K., Kin, Y. S.; Ishida, Y.; Ebina, Y.; Sasaki, T.; Hikima, T.; Aida, T. Photonic Water Dynamically Responsive to External Stimuli. Nat. Commun. 2016, 7, 12559.

(8) Masud, A. R.; Hong, S.-H.; Shen, T.-Z.; Shahzad, A.; Song, J.-K. Electrically and Electrohydrodynamically Driven Phase Transition and Structural Color Switching of Oligomer Tethered 2D Colloid. RSC Adv. 2018, 8, 16549-14556.

(9) Hail, C. U.;Schnoering, G.;Damak, M.;Poulikakos, D.;Eghlidi, H. A Plasmonic Painter's Method of Color Mixing for a Continuous Red-Green-Blue Palette. ACS Nano 2020, 14, 17831791.

(10)Kose, O.; Boott, C. E.; Hamad, W. Y.; MacLachlan, M. J. Stimuli-Responsive Anisotropic Materials Based on Unidirectional Organization of Cellulose Nanocrystals in an Elastomer. Macromolecules 2019, 52, 5317-5324.

(11)Dai, L., Lu, X.; Song, L.; Huang, Y.; Liu, B.; Zhang, L.; Zhang, J.; Wu, S.; Chen, T. Macroscopic-Oriented Gold Nanorods in Polyvinyl Alcohol Films for Polarization-Dependent Multicolor Displays. Adv. Mater. Interfaces 2018, 5, 1800026.

(12)Boott, C. E.; Tran, A.; Hamad, W. Y.; MacLachlan, M. J. Cellulose Nanocrystal Elastomers with Reversible Visible Color. Angew. Chem. 2020, 59, 226-231. 
(13)Cranston, E. D.; Gray, D. G. Birefringence in Spin-Coated Films Containing Cellulose Nanocrystals. Colloids Surf., A 2008, 325, 44-51.

(14)Hausmann, M. K.; Rühs, P. A.; Siqueira, G.; Läuger, J.; Libanori, R.; Zimmermann, T.; Studart, A. R. Dynamics of Cellulose Nanocrystal Alignment During 3D Printing. ACS Nano 2018, 12, 6926-6937.

(15)Cherpak, V.; Korolovych, V. F.; Geryak, R.; Turiv, T.; Nepal, D.; Kelly, J.; Bunning, T. J.; Lavrentovich, O. D.; Heller, W. T.; Tsukruk, V. V. Robust Chiral Organization of Cellulose Nanocrystals in Capillary Confinement. Nano Lett. 2018, 18, 6770-6777.

(16)Song, G.; Kimura, F.; Kimura, T.; Piao, G. Orientational Distribution of Cellulose Nanocrystals in a Cellulose Whisker As Studied by Diamagnetic Anisotropy. Macromolecules 2013, 46, 8957-8963.

(17)Bordel, D.; Putaux, J.-L.; Heux, L. Orientation of Native Cellulose in an Electric Field. Langmuir 2006, 22, 4899-4901.

(18)Zhang, H.; Cadusch, J.; Kinnear, C.; James, T.; Roberts, A.; Mulvaney, P.Direct Assembly of Large Area Nanoparticle Arrays. ACS Nano 2018, 12, 7529-7537.

(19)Hess, A. J.; Funk, A. J.; Liu, Q.; De La Cruz, J. A.; Sheetah, G. H.; Fleury, B.; Smalyukh, I. I. Plasmonic Metamaterial Gels with Spatially Patterned Orientational Order via 3D Printing. ACS Omega 2019, 4, 20558-20563.

(20)Campbell, M. G.;Liu, Q.; Sanders, A.; Evans, J. S.; Smalyukh, I. I. Preparation of Nanocomposite Plasmonic Films Made from Cellulose Nanocrystals or Mesoporous Silica Decorated with Unidirectionally Aligned Gold Nanorods. Materials 2014,7, 3021-3033. 
(21)(a) Huang, H.; Wang, X.; Yu, J.; Chen, Y.; Ji, H.; Zhang, Y.; Rehfeldt, F.; Wang, Y.; Zhang, K.Liquid-Behaviors-Assisted Fabrication of Multidimensional Birefringent Materials from Dynamic Hybrid Hydrogels. ACS Nano 2019, 13, 3867-3874. (b) Yang, Y.; Wang, X.; Huang, H.; Cui, S.; Chen, Y.; Wang, X.; Zhang, K. Modular Nanocomposite Films with Tunable Physical Organization of Cellulose Nanocrystals for Photonic Encryption. Adv. Opt. Mater. 2020, 2000547.

(22)Ye, X.; Zheng, C.; Chen, J.; Gao, Y.; Murray, C. B. Using Binary Surfactant Mixtures to Simultaneously Improve the Dimensional Tunability and Monodispersity in the Seeded Growth of Gold Nanorods. Nano Lett. 2013, 13, 765-771.

(23) (a) Duempelmann, L.; Luu-Dinh, A.; Gallinet, B.; Novotny, L. Four-Fold Color Filter Based on Plasmonic Phase Retarder. ACS Photonics 2016, 3, 190-196. (b) Jiang, M.; Siew, S.H.; Chan, J.Y.E.; Deng, J.; Wu, Q.Y.S.; Jin, L.; Yang, J.K.W.; Teng, J.; Danner, A.; Qiu, C. Patterned Resist on Flat Silver Achieving Saturated Plasmonic Colors with Sub-20-nm Spectral Linewidth. Mater. Today 2020, 35, 99-105. (c) Cheng, L.; Wang, K.; Mao, J.; Goh, X.; Chu, Z.; Zhang, Y.; Zhang, L. Extrinsic Polarization-Enabled Covert Plasmonic Colors Using Aluminum Nanostructures. Ann. Phys. 2019, 531, 1900073. (d) Xie, Z.; Yang, J.; Vashistha, V.; Lee, W.; Chen, K. Liquid-crystal Tunable Color Filters Based on Aluminum Metasurfaces. Opt. Express 2017, 25, 30764-30770.

(24)Carnicer, A.; Javidi, B. Optical Security and Authentication Using Nanoscale and ThinFilm Structures. Adv. Opt. Photon. 2017, 9, 218-256.

(25)Hamad, W. Photonic and Semiconductor Materials Based on Cellulose Nanocrystals. Springer, Cham, 2015, 271, 287-328. 
(26)Liu, J.; Plog, A.; Groszewicz, P.; Zhao, L.; Xu, Y.; Breitzke, H.;Stark, A.; Hoffmann R.;

Gutmann T.; Zhang, K.; Buntkowsky, G. Design of a Heterogeneous Catalyst Based on Cellulose Nanocrystals for Cyclopropanation: Synthesis and Solid-State NMR Characterization. Chem. Eur. J. 2015, 21, 12414-12420.

(27)Reiser, B.; González-García, L.; Kanelidis, I.; Maurer, J. H. M.; Kraus, T. Gold Nanorods with Conjugated Polymer Ligands: Sintering-Free Conductive Inks for Printed Electronics. Chem. Sci. 2016, 7, 4190-4196. 


\section{Publication 3 (Supporting information)}

\section{Structural Colors by Synergistic Birefringence and Surface Plasmon}

\section{Resonance}

Xiaojie Wang, ${ }^{1}$ Dan Xu, ${ }^{1}$ Bea Jaquet, ${ }^{2}$ Yang Yang,,${ }^{1,3}$ Jiaxiu Wang, ${ }^{1}$ Heqin Huang, ${ }^{1}$ Ye Chen, ${ }^{4}$ Christoph Gerhard ${ }^{2}$ and Kai Zhang*1

${ }^{1}$ Wood Technology and Wood Chemistry, Department of Wood Technology and Wood-based Composites, Georg-August-University of Göttingen, Büsgenweg 4, D-37077 Göttingen, Germany

${ }^{2}$ University of Applied Sciences and Arts, Faculty of Engineering and Health, Von-OssietzkyStraße 99, 37085 Göttingen, Germany

${ }^{3}$ State Key Laboratory of Pulp and Paper Engineering, South China University of Technology, Guangzhou, 510640, P. R. China

${ }^{4}$ State Key Laboratory for Modification of Chemical Fibers and Polymer Materials, College of Materials Science and Engineering, Donghua University, Shanghai, 201620, P. R. China

*Corresponding author Email: kai.zhang@uni-goettingen.de

\section{Materials}

Microcrystalline cellulose (MCC), 2,2,6,6-tetramethylpiperidine 1-oxyl (TEMPO), gold (III) chloride trihydrate $\left(\mathrm{HAuCl}_{4} \cdot 3 \mathrm{H}_{2} \mathrm{O}\right.$ ), sodium borohydride (99\%), 3-(acrylamido)phenylboronic acid (PBA), L-ascorbic acid (99\%), hexadecyltrimethylammonium bromide (CTAB, 99\%), methacrylic anhydride, phosphotungstic acid hydrate, 2,4,6-trimethylbenzoyl chloride were all purchased from Sigma-Aldrich (USA). Dopamine hydrochloride, poly(styrene sulfonic acid) sodium salt (PSS, M.W. 70000), sodium hypochlorite solution (11-15\%), dimethyl 
phenylphosphonite and 2-butanone were provided by Alfa Aesar (USA). Sodium oleate (NaOL, 97\%) was supplied by TCI (Japan). Sodium sulfate anhydrous, disodium tetraborate, Acrylamide (Am) were obtained from Merck Millipore (USA). Sodium hydroxide and silver nitrate $\left(\mathrm{AgNO}_{3}\right)$ were ordered from VWR (Germany). Sodium bicarbonate was bought from TH Geyer (Germany). Hydrochloric acid (37\%) was obtained from AppliChem (Germany). The organic solvents, including tetrahydrofuran (THF), ethyl acetate, n-hexane and acetone were all obtained from TH Geyer (Germany). Dopamine methylacrylate (DMA) ${ }^{1}$ and lithium phenyl-2,4,6-trimethylbenzoylphosphinate (LAP) ${ }^{2}$ were synthesized according to previously reported methods. Deionized water utilized throughout all experiments was purified from a Millipore system.

\section{Methods}

1. Transmission, reflection and haze measurement

In order to gain information on the optical properties of the samples, transmission, reflection and haze were determined. The first two parameters were measured with the aid of an UV-VIS spectrometer (Lambda 650 from Perkin Elmer), where the considered wavelength range was $300-800 \mathrm{~nm}$ and the increment was $5 \mathrm{~nm}$. Transmission was detected at normal incidence of the measuring light beam whereas reflection measurements were carried out at an angle of incidence of $8^{\circ}$. The particular values presented here are the mean values of three single measurements.

The haze as the reflection haze $\mathrm{e}^{3,4}$ was determined according to the standard ASTM E430,6 using a commercial measurement device (Novo-Gloss 20/60/85 Glossmeter from Rhopoint Instruments). This parameter was detected at five different measuring points with a spacing of approximately $1 \mathrm{~mm}$. The presented data are the mean values obtained by this procedure.

\section{Mechanical properties test}

A Z3 micro tensile test machine (Thümler GmbH, Germany) equipped with a $50 \mathrm{~N}$ load cell was used for uniaxial tensile tests. The crosshead speed is $2.5 \mathrm{~mm} / \mathrm{min}$. The nominal tensile 
stress and strain were recorded for the calculation of the ultimate tensile strength and elongation at break.

Supporting Figures S1-S14 and Table S1.

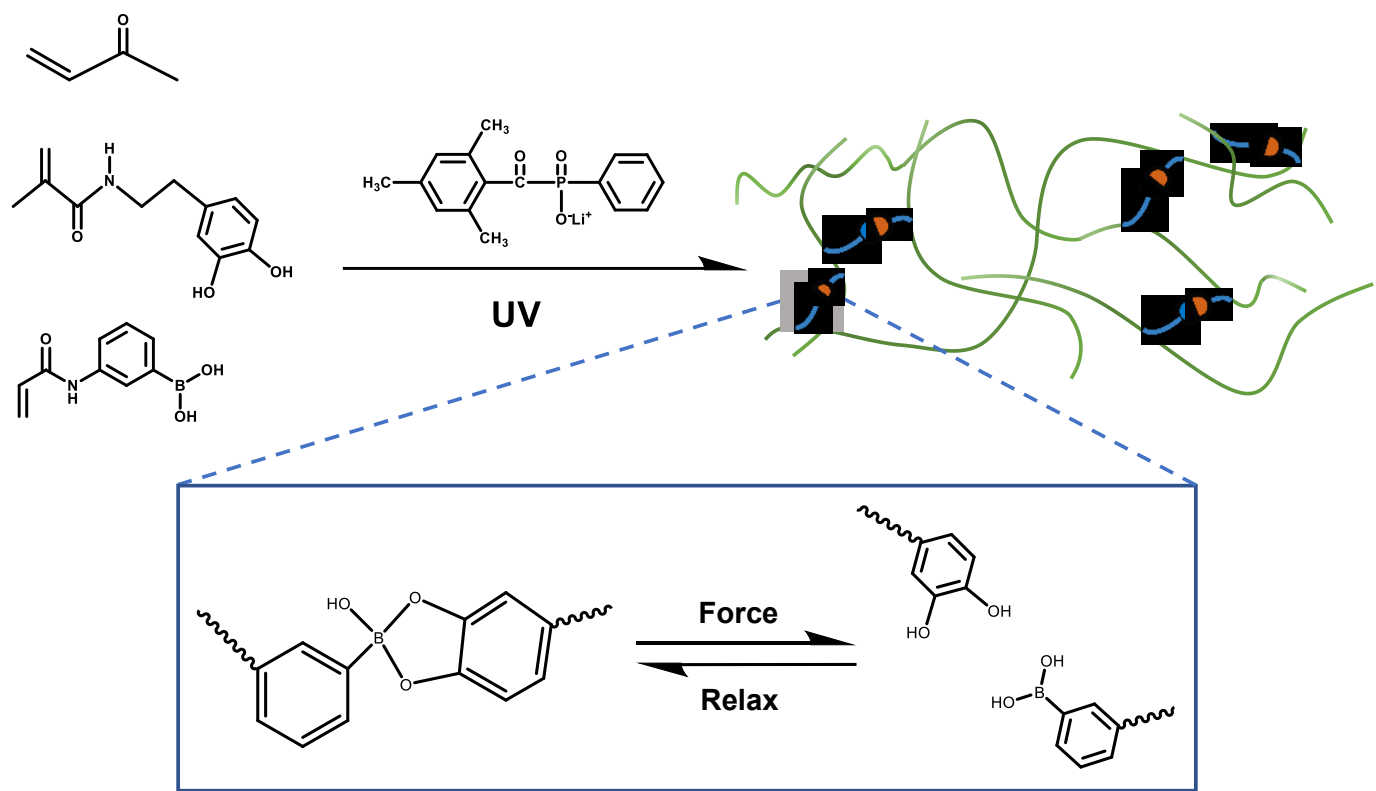

Figure S1. Schematic illustration for the formation of dynamic crosslinked networks in poly(acrylamide) (f-PAm) films.

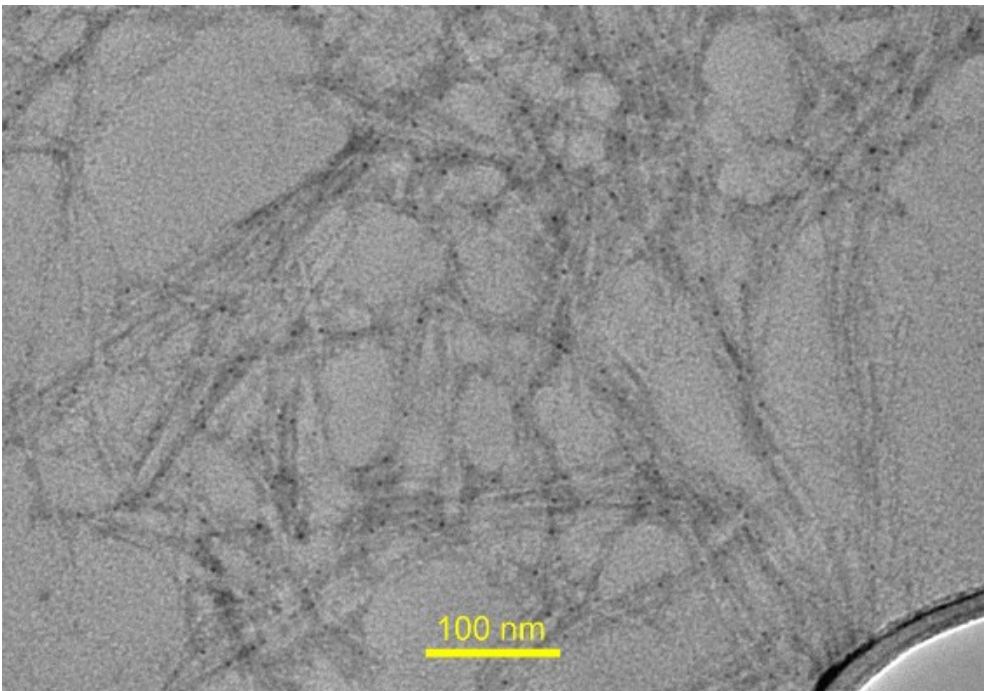

Figure S2. A representative TEM image of TEMPO-mediated oxidized CNCs. 
(a)

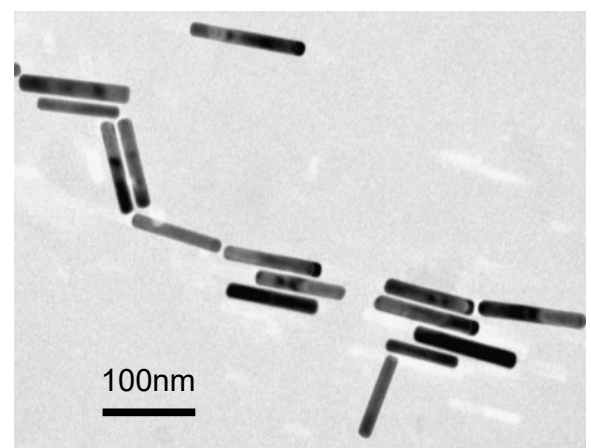

(b)

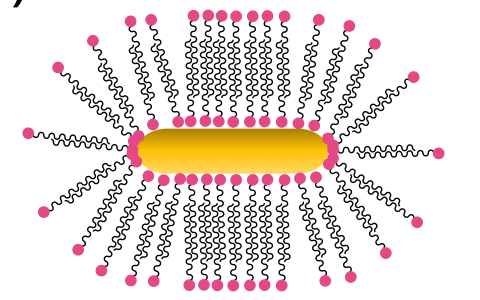

Cetyltrimethylammonium

bromide(CTAB) capped

$($ Zeta potential $+52.7 \mathrm{mV}$ )

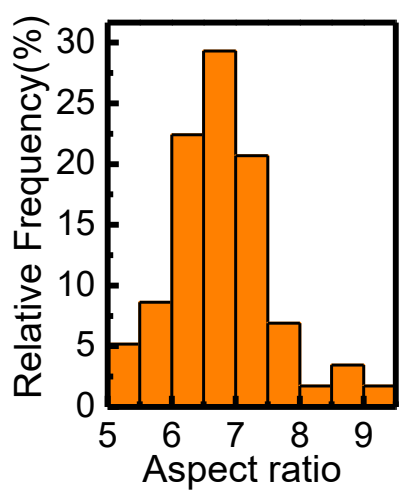

Ligand exchange
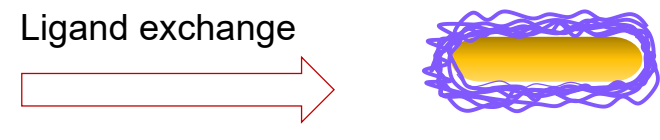

Poly(styrenesulfonate)

(PSS) capped

(Zeta potential -34.2 mV)

Figure S3. (a) A representative TEM image of CTAB-GNRs and their aspect ratios; (b) Schematic illustration for the ligand exchange of CTAB-GNRs to PSS-GNRs.

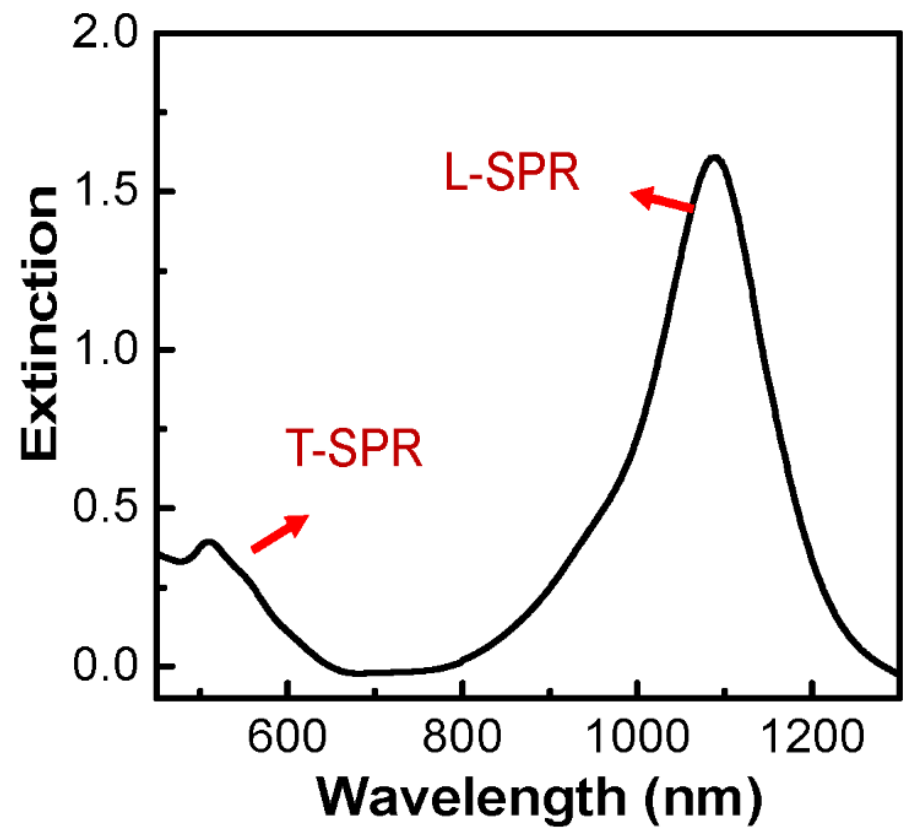

Figure S4. UV-Vis-NIR extinction spectrum of the aqueous GNRs dispersion. 


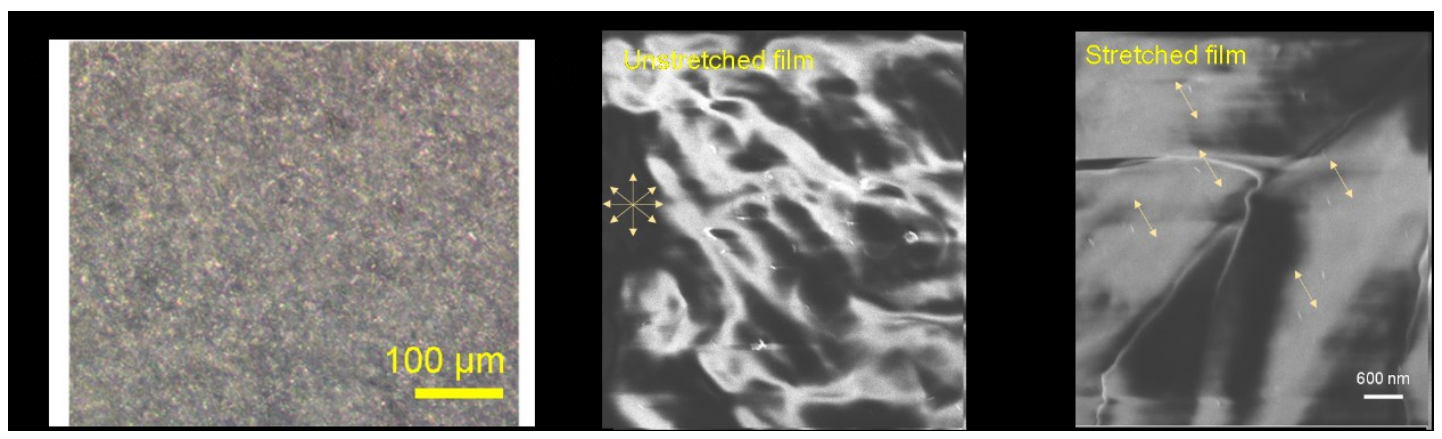

Figure S5. (a) The POM image of hydrogel precursor solutions with pre-mixed CNCs1/GNRs1. (b) SEM images showing the distribution of gold nanorods in unstretched and stretched film at lower magnification. Arrows in the right panel indicate the orientation of GNRs along the stretching direction. the stretching. The images were obtained by directly observing the specimens in SEM. The concentration of GNR in both specimens is $1 \mathrm{wt} \%$.

The SEM images of unstretched and stretched hybrid films with lower magnification are shown in Figure S5. The GNRs in unstretched film are disordered, while they are well aligned in stretched films.
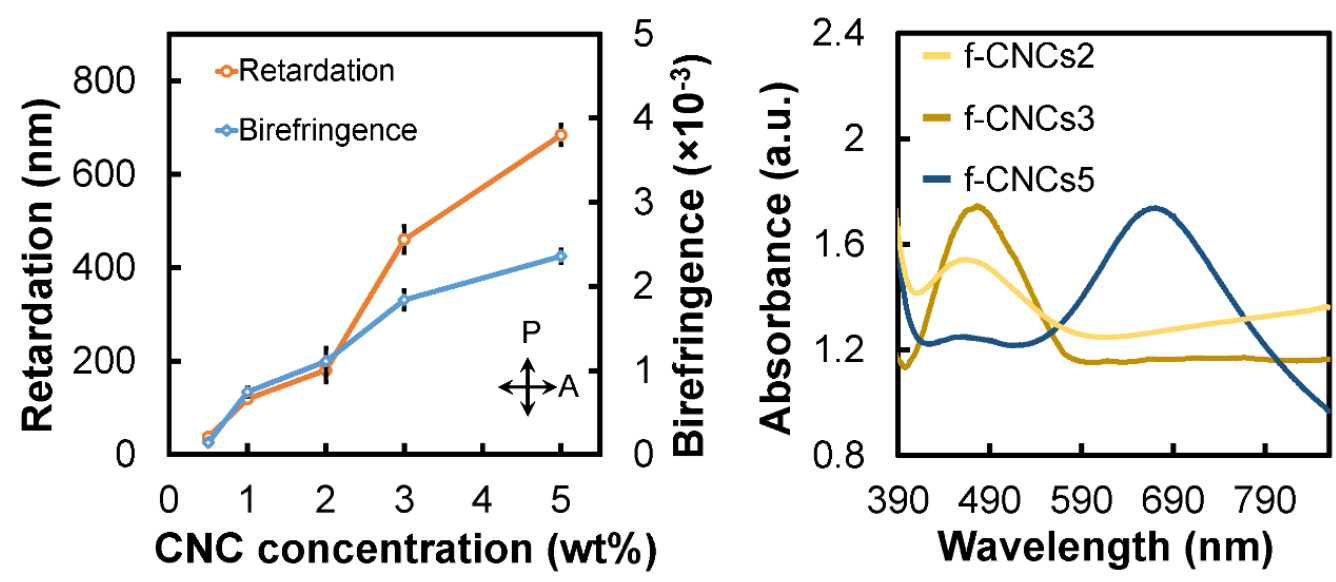

Figure S6. Light retardation and birefringence (left panel) and UV-Vis spectra (right panel) of f-CNCs films with various CNCs concentrations. 


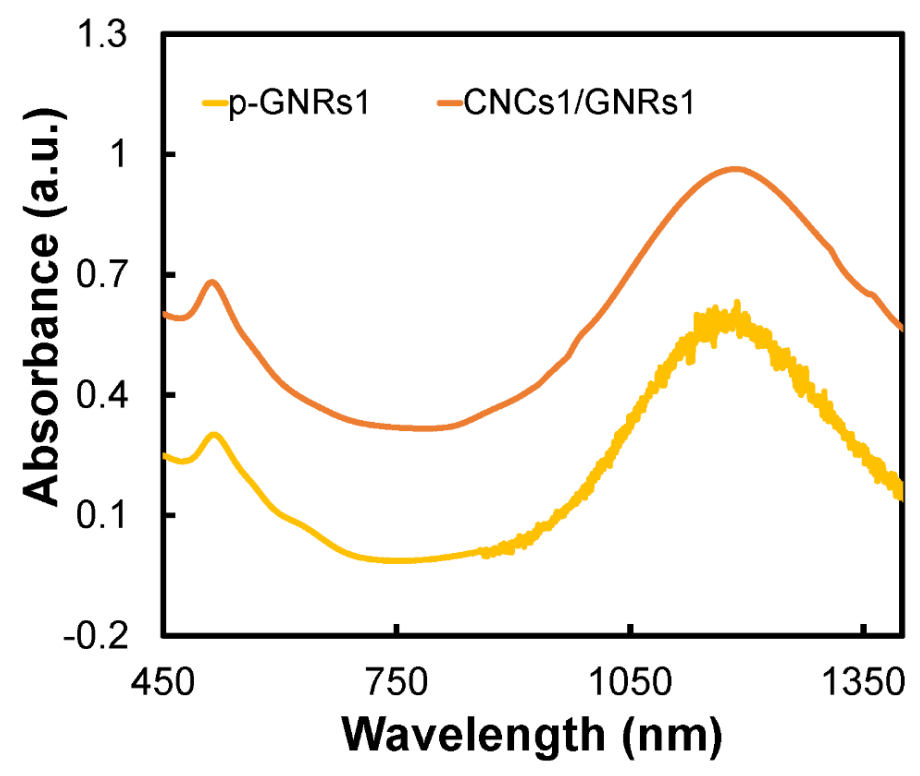

Figure S7. UV-Vis-NIR spectra of f-GNRs1 and CNCs1/GNRs1 composite films.

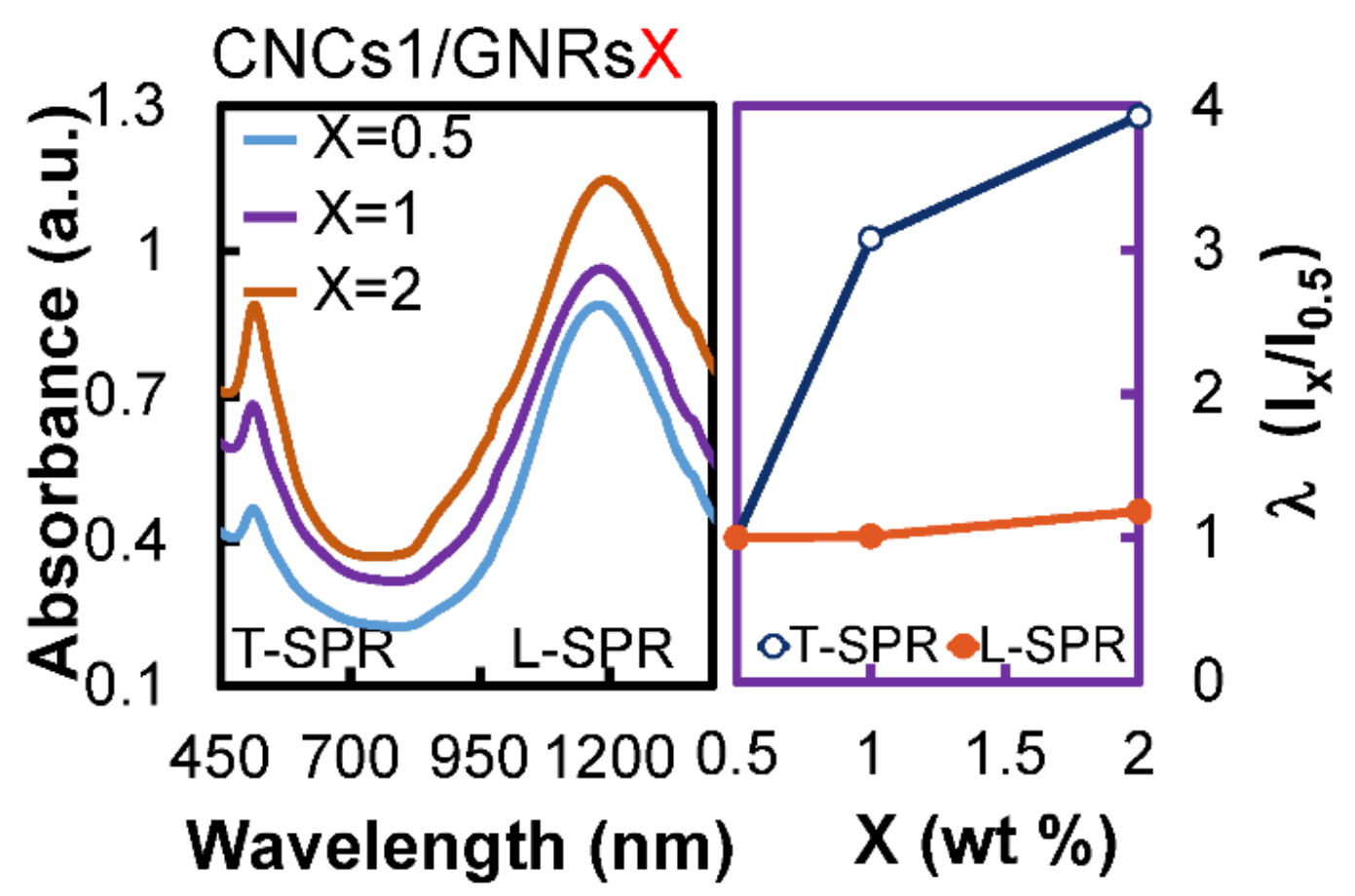

Figure S8. UV-Vis-NIR spectra and the curve integrals of CNCs1/GNRsX hybrid films that were placed $45^{\circ}$ against one polarizer. Ix: Intensity of absorption peaks with various GNRs contents X. 


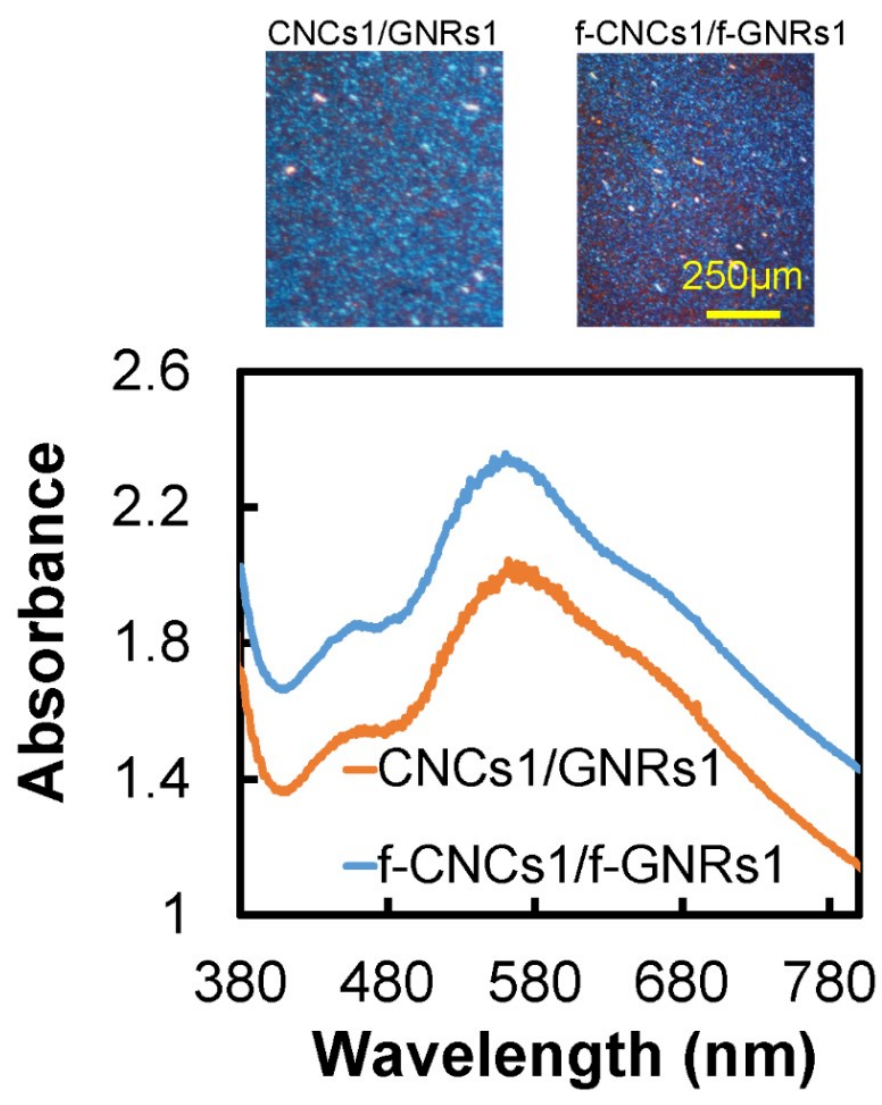

Figure S9. Structural colors and the UV-Vis spectra of CNCs1/GNRs1 hybrid composite film and stacked f-CNCs1/f-GNRs1films.

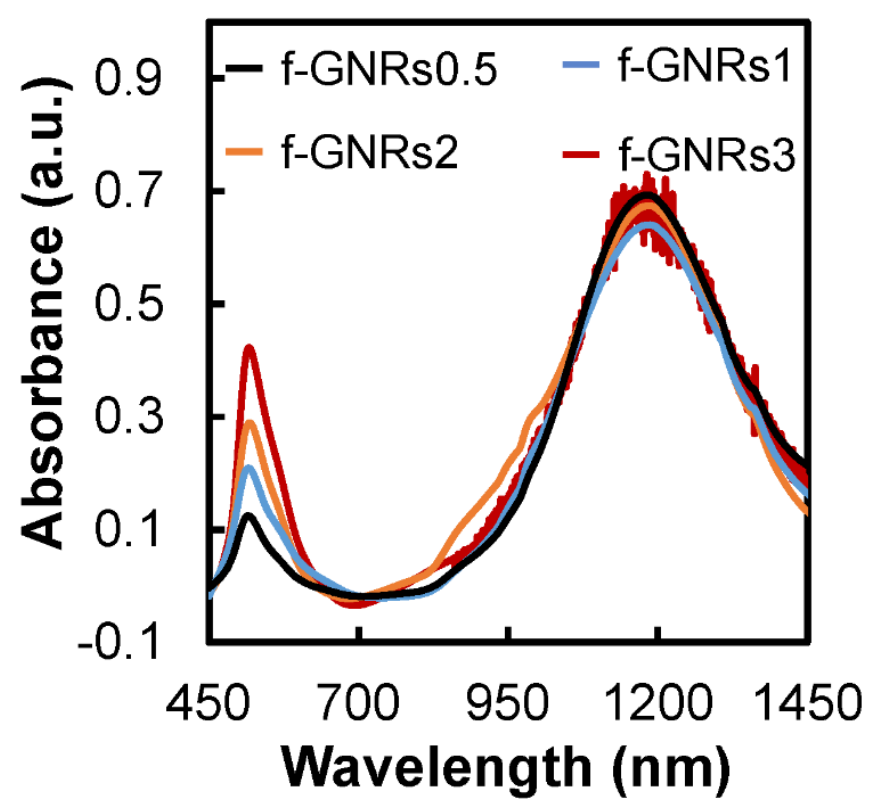

Figure S10. UV-Vis-NIR spectra of various f-GNRs composite films measured with one polarizer. 


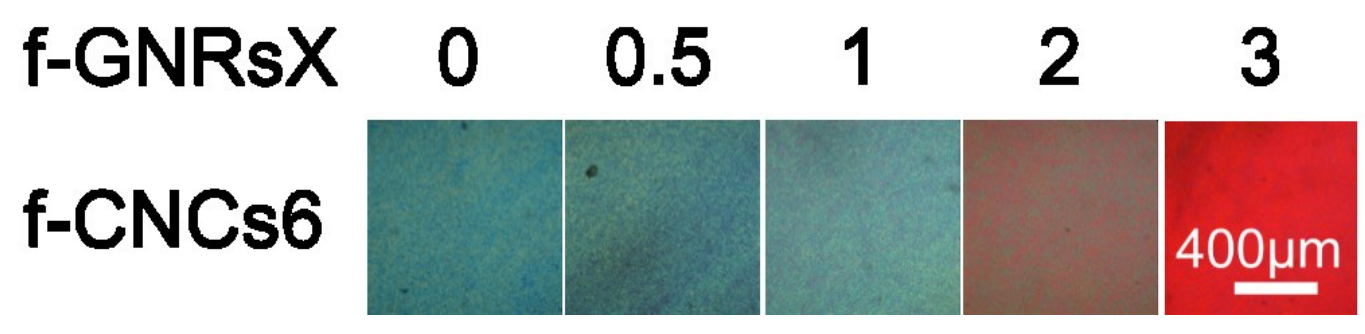

Figure S11. Color evolution of two piled f-CNCs3 films (referred to as f-CNCs6 in Figure) with additional various f-GNRsX films from green to red.
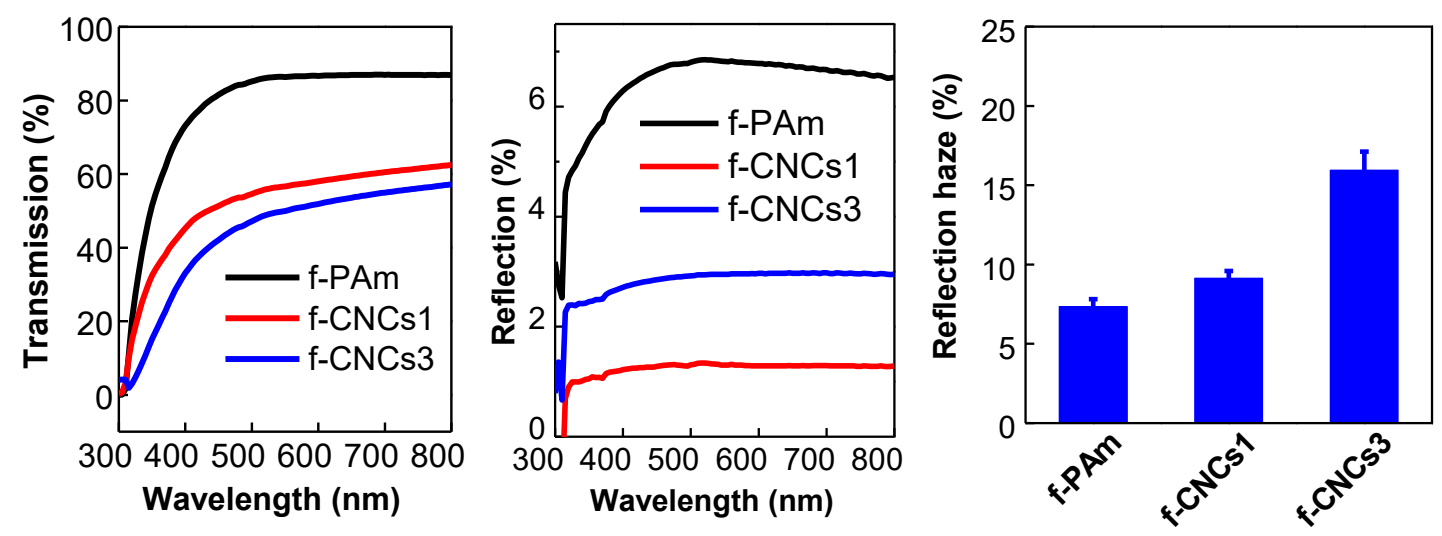

Figure S12. Transmission and reflection curves including reflection haze of representative CNCs films and films without CNCs (f-PAm).

The transmission and reflection of the representative CNCs films (f-CNCs1 and f-CNCs3) and poly(acrylamide) films (f-PAm) to demonstrate optical properties for those films, as shown in Figure S12.

The incorporation of CNCs largely decreased the transmission of films from maximally $\sim 85 \%$ to less than $60 \%$ compared with f-PAm films. Moreover, the light reflection of CNCscontaining films also significantly reduced from max. $7 \%$ to less than $3 \%$ compared to f-PAm. On the contrary, the haze values of f-CNCs films are generally higher than f-PAm films with the haze of around $7.3 \%$. The $\mathrm{f}-\mathrm{CNCs} 3$ reached a haze of $16 \%$ and a lower concentration of $\mathrm{CNCs}$ in $\mathrm{f}-\mathrm{CNCs} 2$ resulted in a lower haze of around 9\%. These results indicate that the addition of $\mathrm{CNCs}$ decreased the light reflection and transmission, and a semi-transparent property due to increasing haze. 

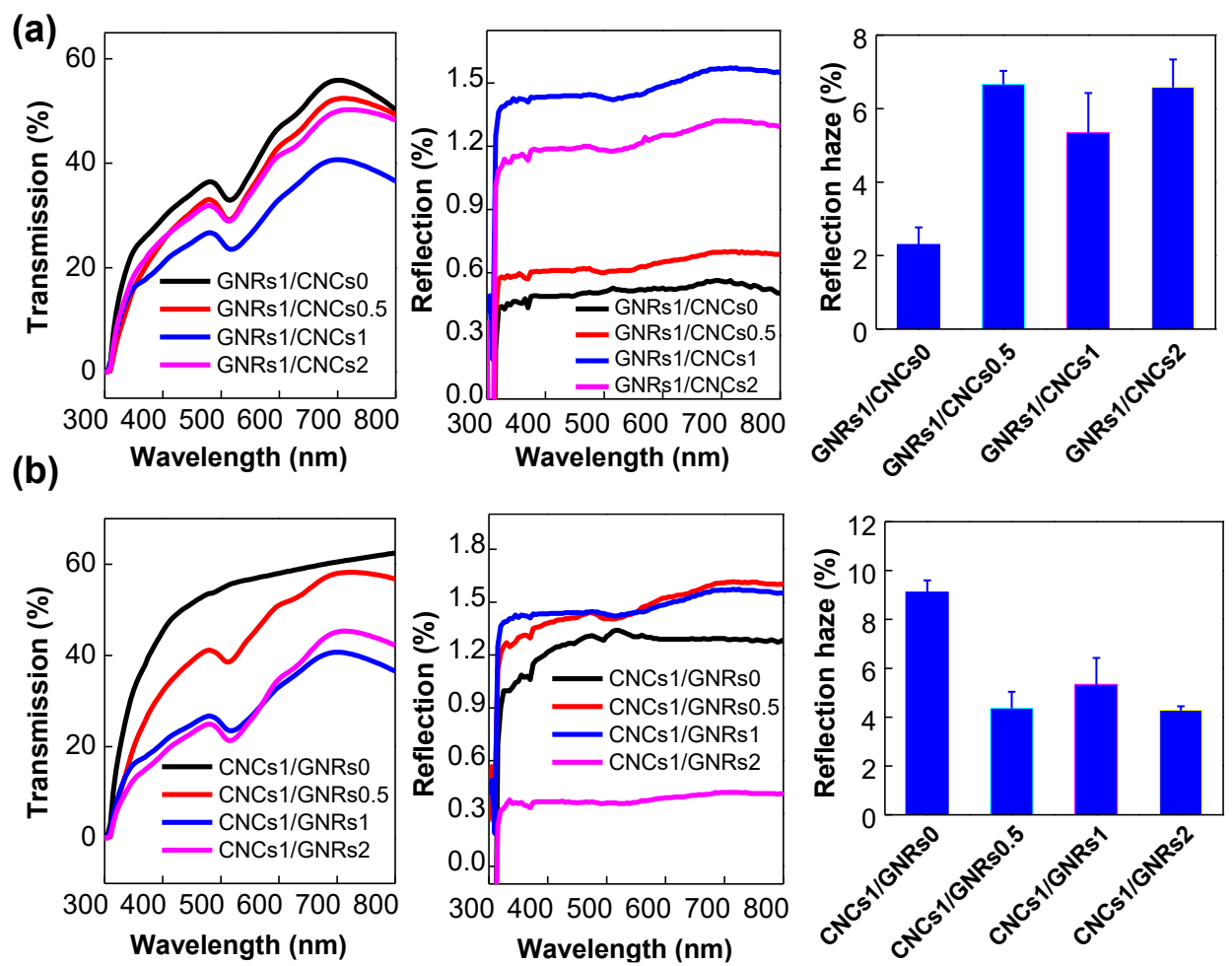

Figure S13. Transmission and reflection curves of CNCs/GNRs hybrid films with (a) increasing contents of CNCs ( $\mathrm{Y}=\mathbf{0 - 2} \mathrm{wt} \%$ in initial hydrogel precursor solutions) or (b) GNRs (X=0-2 wt\% in initial hydrogel precursor solutions), respectively.

With the fixed content of GNRs at $1 \mathrm{wt} \%$, and increasing CNCs concentration in hybrid films, the films showed lower transmission (Figure S13a). In accordance, the reflected light increased, but the reflection intensity is generally very low (less than 1.6\%). At the same time, CNCs1/GNR0 showed a haze of around 9\%, while the addition of GNRs into the films decreased the haze to around $5 \%$.

In a similar manner, at fixed CNCs content of $1 \mathrm{wt} \%$, increasing GNRs contents also decreased the intensity of transmitting light (Figure S13b). In accordance, the reflected light increased, but the reflection intensity is generally highly limited (less than 1.6\%). Moreover, GNR1/CNCs0 showed a haze of around $2.4 \%$, while the presence of CNCs in the films increased the haze to $5 \%-7 \%$. 


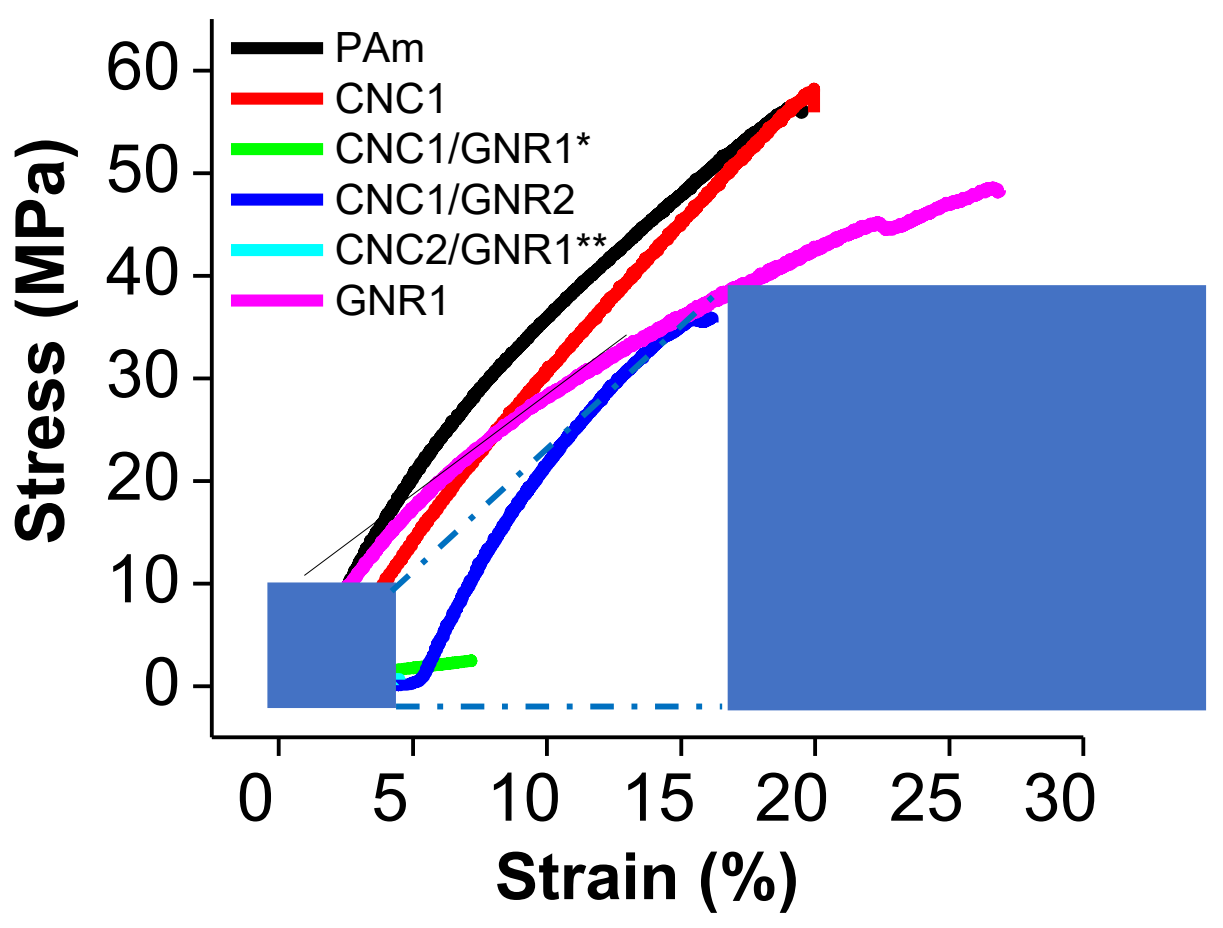

Figure S14. The mechanical properties of various films. $(*, * *$ due to the brittleness of samples and the defect of fixture, these data could be not reliable)

According to the uniaxial tensile tests, the hybrid polymer films (GNR1) have high elastic modulus, which could make up to ca. $0.3 \mathrm{GPa}$. Compared to pure f-PAm films with the elastic modulus less than $0.2 \mathrm{GPa}$, the existence of CNC and GNR in hybrid films could enhance the mechanical properties to a certain degree. In addition, the hybrid films became brittle with increasing contents of $\mathrm{CNC} / \mathrm{GNRs}$. 
Table S1. Contents of nanoparticles in hydrogel precursors and dried films.

\begin{tabular}{cccccc}
\hline $\begin{array}{c}\text { Hydrogel } \\
\text { precursor } \\
(2 \mathrm{~mL})\end{array}$ & AAm $(\mathrm{mg})$ & $\begin{array}{c}\text { PBA } \\
(\mathrm{mg})\end{array}$ & $\begin{array}{c}\text { DMA } \\
(\mathrm{mg})\end{array}$ & $\begin{array}{c}\text { LAP } \\
(\mathrm{mg})\end{array}$ & $\begin{array}{c}\text { Nanoparticles content in } \\
\text { dried film }\end{array}$ \\
\hline $\begin{array}{c}\text { GNRs } 1 \mathrm{wt} \% \\
(20 \mathrm{mg})\end{array}$ & 288 & 9.6 & 11 & 10 & $5.9 \mathrm{wt} \%$ \\
$\mathrm{CNCs} 1 \mathrm{wt} \%$ & 288 & 9.6 & 11 & 10 & $5.9 \mathrm{wt} \%$ \\
$(20 \mathrm{mg})$ & & & & & \\
\hline
\end{tabular}

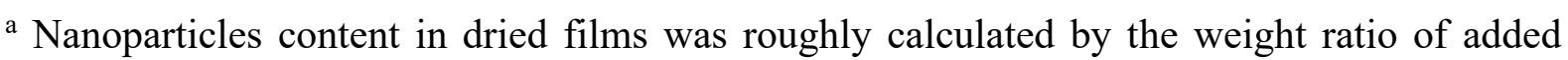
nanoparticles and the added weight of all the solid components in hydrogel precursors.

\section{References}

(1) Lee, H.; Lee, B. P.; Messersmith, P. B. A Reversible Wet/Dry Adhesive Inspired by Mussels and Geckos. Nature 2007, 448, 338-341.

(2) Fairbanks, B. D.; Schwartz, M. P.; Bowman, C. N.; Anseth, K. S. Photoinitiated Polymerization of PEG-Diacrylate with Lithium Phenyl-2,4,6-trimethylbenzoylphosphinate: Polymerization Rate and Cytocompatibility. Biomaterials 2009, 30, 6702-6707.

(3) G. Kigle-Boeckler: Measurement of gloss and reflection properties of surfaces, Metal Finishing 1995, 93, 28-31.

(4) R. Alexander-Katz and R. G. Barrera; Surface correlation effects on gloss, Journal of Polymer Science B 1998, 36, 1321-1334.

(5) ASTM E430-11, Standard Test Methods for Measurement of Gloss of High-Gloss Surfaces by Abridged Goniophotometry, ASTM International, West Conshohocken, PA, 2011.

(6) Westlund, H. B.; Meyer, G. W., Applying appearance standards to light reflection models. In Proceedings of the 28th annual conference on Computer graphics and interactive techniques, Association for Computing Machinery: 2001, 501-510. 


\section{Curriculum vitae}

\section{PERSONAL DETAILS}

Surname, Name: Wang, Xiaojie

Gender: Female

Nationality: Chinese

Date of Birth: 24.11.1990

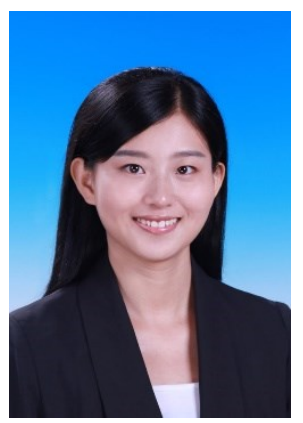

Email: xwang7@uni-goettingen.de; wang.xiaojie@hotmail.com

\section{EDUCATIONAL BACKGROUND}

Feb. 2017 - Jul. 2020

Georg-August-Universität Göttingen (Germany), Wood Technology and Wood Chemistry, graduated in July 2020 with a Doctoral Degree (Dr. rer. nat.).

Sep. 2013 - Jul.2016

Beijing University of Chemical Technology (China), Materials Science and Engineering, graduated in July 2016 with a Master Degree.

Sep. 2009 - Jun. 2013

University of Jinan (China), Polymer Materials and Engineering, graduated in June 2013 with a Bachelor Degree.

\section{PUBLICATIONS}

1. YangYang, Xiaojie Wang, Heqin Huang, Shiqiang Cui, Ye Chen, Xiaohui Wang, Kai Zhang. Modular nanocomposite films with tunable physical organization of cellulose nanocrystals for photonic encryption. Advanced Optical Materials, 2020, 2000547.

2. Minshen Zhu\#, Xiaojie Wang\#, Hongmei Tang, Jiawei Wang, Qi Hao, Lixiang Liu, Yang Li, Kai Zhang, Oliver G. Schmidt. Antifreezing hydrogel with high zinc reversibility for 
flexible and durable aqueous batteries by cooperative hydrated cations. Advanced Functional Materials, 2019, DOI: 10.1002/adfm.201907218. (\# equal contribution)

3. Heqin Huang, Yang Yang, Xiaojie Wang, Florian Rehfeldt, and Kai Zhang. Thermoresponsive water transportation in dually electrostatically crosslinked nanocomposite hydrogels. Macromolecular Rapid Communications, 2019, 40, 1900317.

4. Heqin Huang, Xiaojie Wang, Jinchao Yu, Ye Chen, Hong Ji, Yumei Zhang, Florian Rehfeldt, Yong Wang, and Kai Zhang. Liquid-behaviors-assisted fabrication of multidimensional birefringent materials from dynamic hybrid hydrogels. ACS Nano, 2019, DOI: $10.1021 /$ acsnano.9b00551.

5. Xiaojie Wang, Heqin Huang, Huan Liu, Florian Rehfeldt, Xiaohui Wang, and Kai Zhang. Multi-responsive bilayer hydrogel actuators with programmable and precisely tunable motions. Macromolecular Chemistry and Physics, 2019, 220, 1800562.

6. Heqin Huang, Xiaojie Wang, Florian Rehfeldt, and Kai Zhang. Dually heterogeneous hydrogels via dynamic and supramolecular cross-links tuning discontinuous spatial ruptures. ACS Sustainable Chemistry \& Engineering, 2018, 6 (3), 4294-4301.

7. Yonggui Wang, Xiaojie Wang, Yanjun Xie, Kai Zhang. Functional nanomaterials through esterification of cellulose: a review of chemistry and application. Cellulose, 2018, 25(7), 3703-3731.

8. Heqin Huang, Yonggui Wang, Xiaojie Wang, Florian Rehfeldt, and Kai Zhang. Robust heterogeneous hydrogels with dynamic nanocrystal-polymer interface. Macromolecular Rapid Communications, 2017, 38, 1600810.

9. Xiaojie Wang, Chen Zhang, Zhongjie Du, Hangquan Li,Wei Zou. Synthesis of nondestructive amido group functionalized multi-walled carbon nanotubes and their application in antistatic and thermal conductive polyetherimide matrix nanocomposites. Polymers for Advanced Technologies, 2016, 28 (7), 791-796. 